\title{
EXPERIMENTAL ANALYSIS AND NUMERICAL MODELING \\ OF PARTICLE EMBEDMENT AND FRACTURE IN \\ THE SOLID PARTICLE EROSION OF DUCTILE MATERIALS
}

by

\section{Vahid Hadavi}

MSc , Aero Space Engineering, K.N.Toosi University of Technology, Iran, 2008 BSc , Mechanical Engineering, Kerman University, Iran, 2005

\author{
A dissertation \\ presented to Ryerson University \\ in partial fulfillment of the \\ requirement for the degree of \\ Doctor of Philosophy \\ in the Program of \\ Mechanical Engineering \\ Toronto, Ontario, Canada, 2016 \\ (C) Vahid Hadavi 2016
}




\section{AUTHOR'S DECLARATION FOR ELECTRONIC SUBMISSION OF DISSERTATION}

I hereby declare that I am the sole author of this dissertation. This is a true copy of the dissertation, including any required final revisions, as accepted by my examiners.

I authorize Ryerson University to lend this dissertation to other institutions or individuals for the purpose of scholarly research.

I further authorize Ryerson University to reproduce this dissertation by photocopying or by other means, in total or in part, at the request of other institutions or individuals for the purpose of scholarly research.

I understand that my dissertation may be made electronically available to the public. 


\title{
Abstract
}

\section{Experimental Analysis and Numerical Modeling of Particle Embedment and Fracture in the Solid Particle Erosion of Ductile Materials}

\author{
Vahid Hadavi \\ Doctor of Philosophy, 2016 \\ Mechanical Engineering \\ Ryerson University
}

Embedment and fracture of abrasives are two often neglected important phenomena that can affect material removal occurring in industrial processes that involve high speed impact of particles on relatively ductile targets. This thesis proposes new methodologies to predict the likelihood of particle embedment and fracture for a typical solid particle erosion application.

Double-pulsed laser shadowgraphy was used to measure the instantaneous orientation of angular 89-363 $\mu \mathrm{m} \mathrm{SiC} \mathrm{particles} \mathrm{within} \mathrm{a} \mathrm{micro-abrasive} \mathrm{jet,} \mathrm{in} \mathrm{order} \mathrm{to} \mathrm{assess} \mathrm{whether} \mathrm{their}$ orientation affected the propensity for particle embedment. A tendency for particles to orient with the jet axis was measured and successfully modelled ( $<9 \%$ error), with larger abrasives more likely to orient. The measured instantaneous orientation of particles was used to generate a threedimensional coupled finite element and smoothed particle hydrodynamics model capable of simulating the particle embedment. Use of various combinations of process parameters yielded embedment predictions that agreed with measured ones with, at most, a 16\% error. Increases in particle size, orientation angle, and velocity were found to enhance the propensity for embedment.

Double-pulsed laser shadowgraphy was used to record the impact and fracture of abrasives upon impact. A numerical model that utilized an Element Free Galerkin (EFG) technique with a novel scheme for generating realistic three-dimensional particle geometries was used to simulate the particle fracture. For a wide variety of process parameters, the numerical predictions of particle average size, roundness and rebound velocity agreed with the corresponding measurements to within $10 \%$, at most. The propensity for particle fracture was found to depend on the magnitude of particle kinetic energy perpendicular to the target. It was confirmed that at the same incident 
velocity, larger particles were more likely to fracture. However, for the same kinetic energy, smaller particles were more likely to fracture.

To the best knowledge of the author, this thesis is the first to report measurements of particle orientation and particle fracture in abrasive jets, and the first to develop numerical modeling of particle fracture and embedment. The results have important implications for erosion testing and abrasive jet machining operations. 


\section{Acknowledgements}

I would like to thank:

$>$ Dr. Marcello Papini for providing me with the opportunity of joining his research team and his persistent and friendly support, encouragement, patience and advices in all steps of my $\mathrm{PhD}$.

$>$ The financial support of the Natural Sciences and Engineering Research Council of Canada (NSERC) and the Canada Research Chairs Program.

$>$ Ryerson University for the fantastic facilities and equipment provided for this research.

$>$ Alan Machin, Qiang Li, Chao Ma, Grace He and Joseph Amankrah for their technical support.

> My valued colleagues Björn Michaelsen and Carlos E. Moreno for their excellent assistance.

$>\quad$ My good friends Dr. Reza Jafar, Dr. Mahdi Takaffoli, Dr. Naser Haghbin and Ali Nouhi for their valuable consultations.

$>\quad$ My dear friends Dr. Farhang Sahba and Mrs. Niloufar Alemzadeh and their family for their valuable support since the first day I arrived in Canada 


\section{Dedication}

This dissertation is dedicated to my beloved parents, my dear uncle Dr. Hossein Jaffarian, and all my loved ones. 


\section{Table of Contents}

AUTHOR'S DECLARATION FOR ELECTRONIC SUBMISSION OF DISSERTATION ....... ii

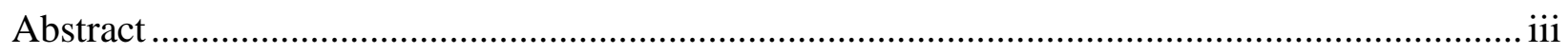

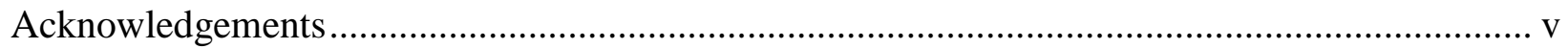

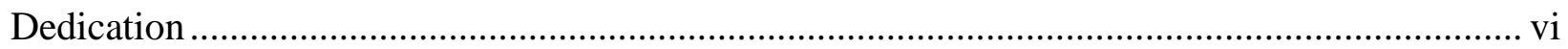

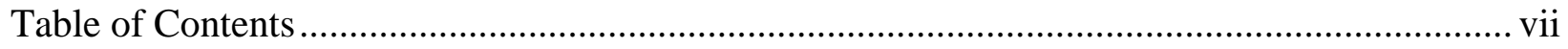

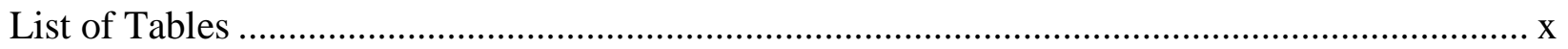

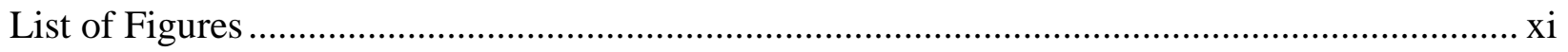

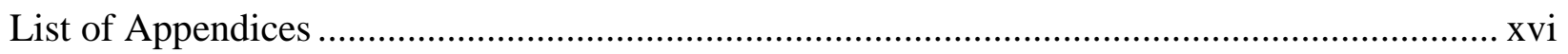

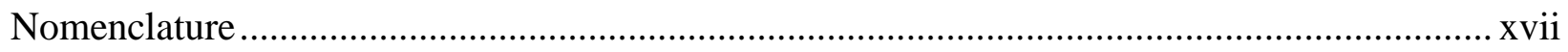

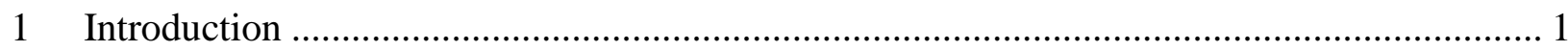

1.1 The role of particle embedment and fracture in solid particle erosion.......................... 1

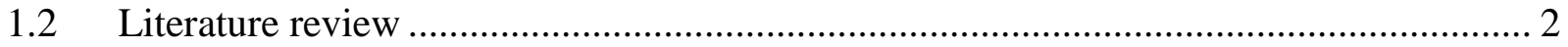

1.2.1 Particle embedment in erosive processes ........................................................ 2

1.2.2 Particle orientation in a flow .......................................................................... 5

1.2.3 Abrasive particle fracture due to impact............................................................ 6

1.2.4 Numerical simulation of particle fracture in solid particle erosion ........................... 9

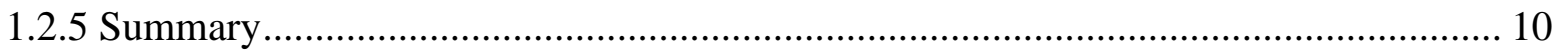

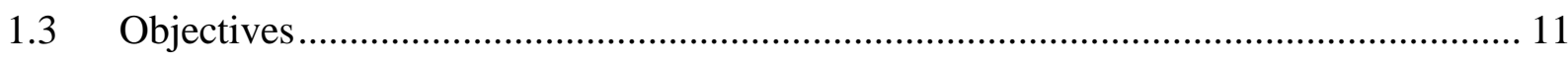

2 Measurements and modeling of instantaneous particle orientation within abrasive air jets

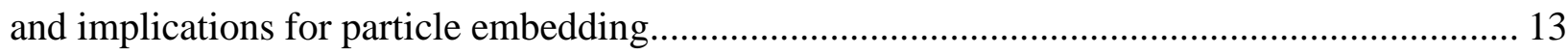

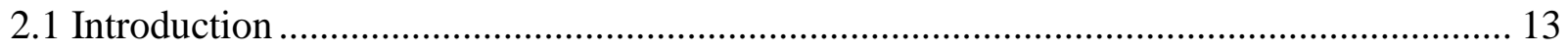

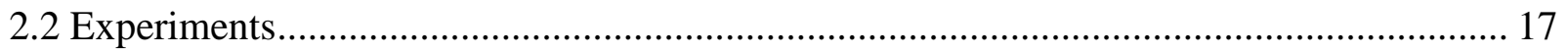

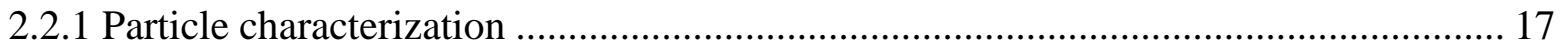

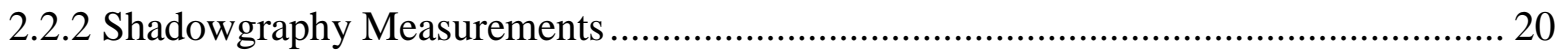

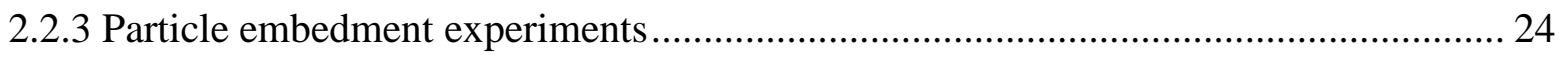

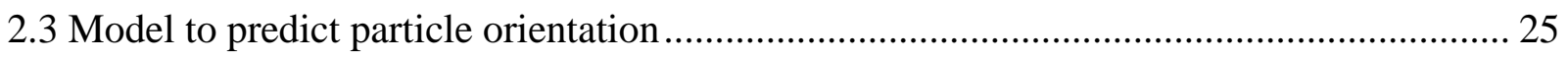

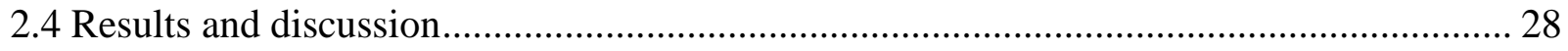

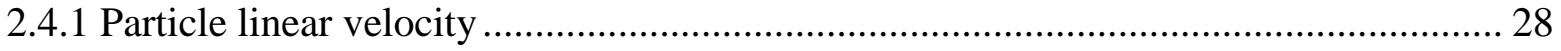




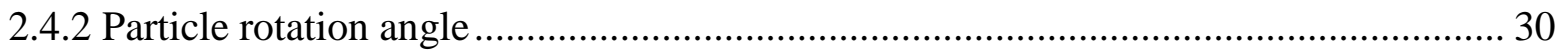

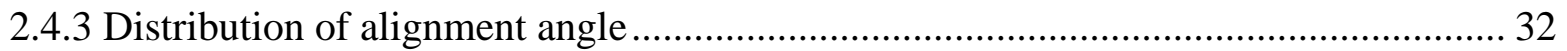

2.4.4 Influence of process parameters on the distribution of orientation angle..................... 33

2.4.5 Comparison between predicted and measured particle orientation .............................. 35

2.5 Influence of particle orientation on particle embedding ................................................... 37

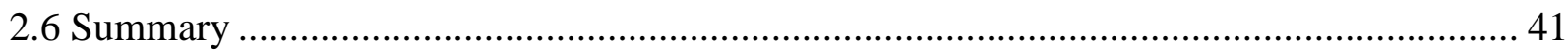

3 Numerical Modeling of Particle Embedment during Solid Particle Erosion of Ductile

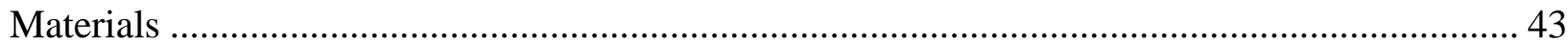

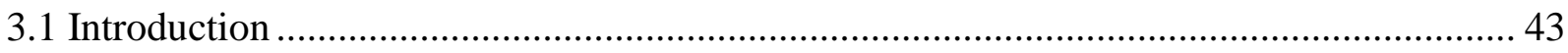

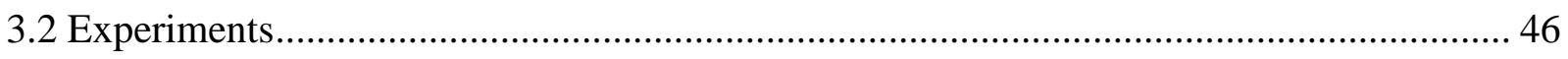

3.2.1 Measurement of particle embedment …………….................................................. 46

3.2.2 Measurement of particle orientation and velocity ..................................................... 49

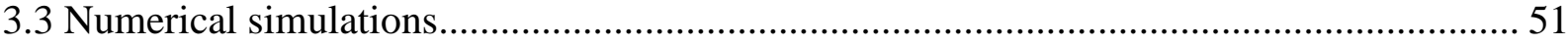

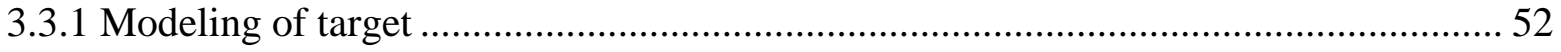

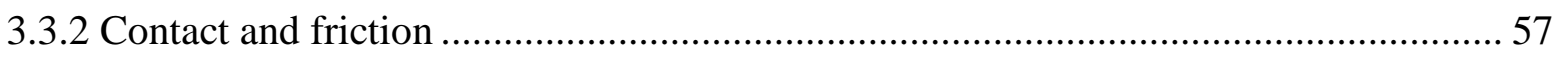

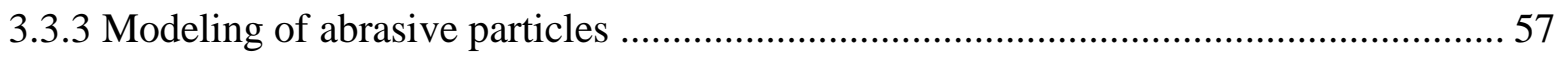

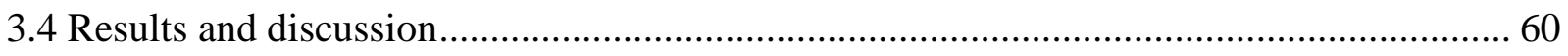

3.4.1 Determination of embedment in numerical models .................................................... 60

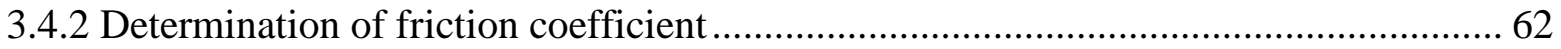

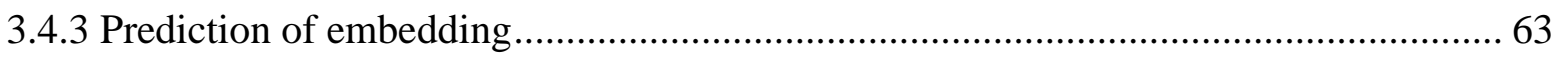

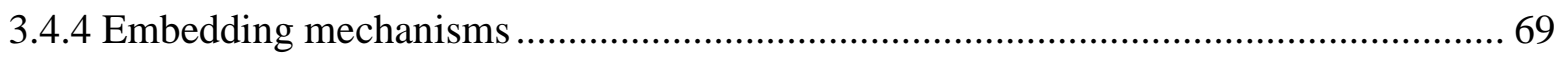

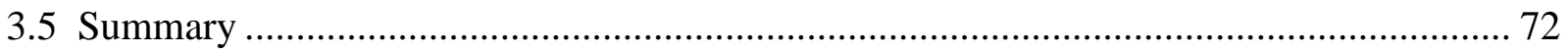

4 Numerical Modeling and Experimental Analysis of Particle Fracture During Solid Particle

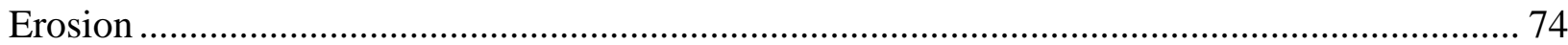

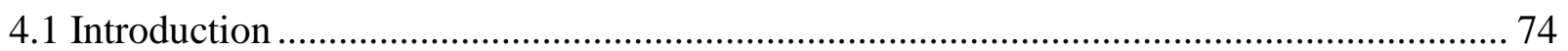

4.1.1 Abrasive particle fracture due to impact................................................................... 74

4.1.2 Numerical modeling of fracture due to impact......................................................... 76

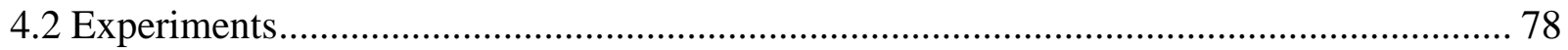

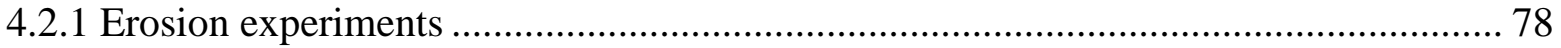

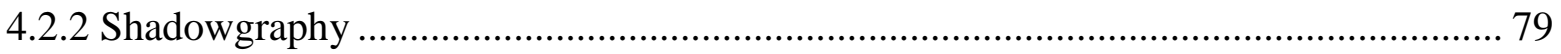

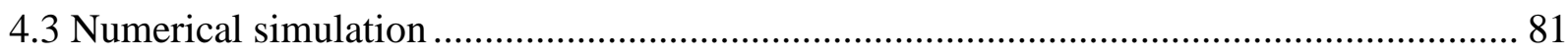

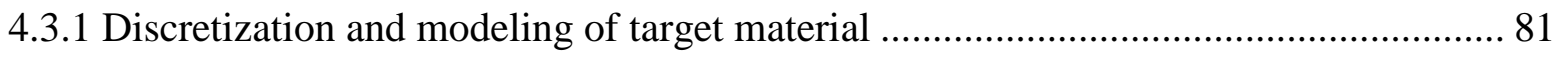




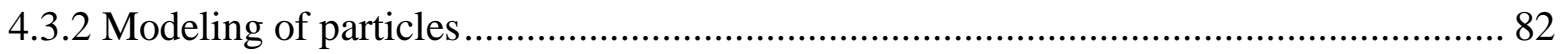

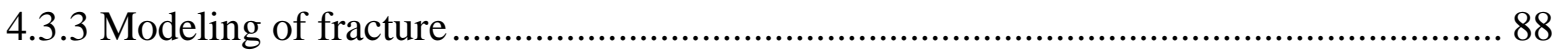

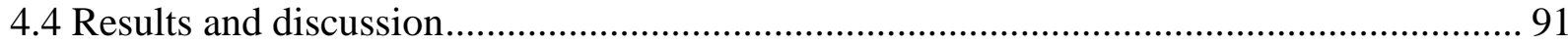

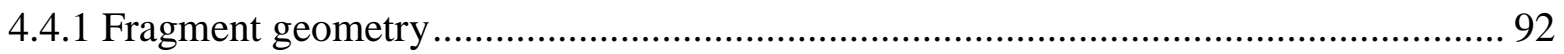

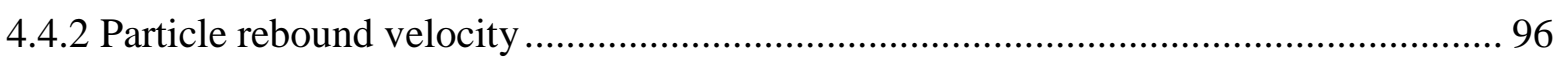

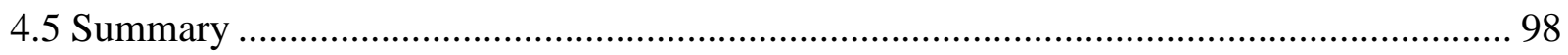

5 Numerical and Experimental Investigation of the Influence of Process Parameters on Particle Fracture during Solid Particle Erosion ................................................................... 99

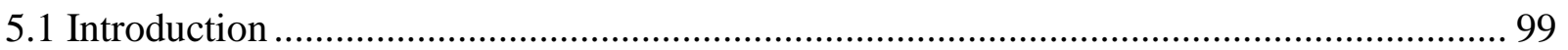

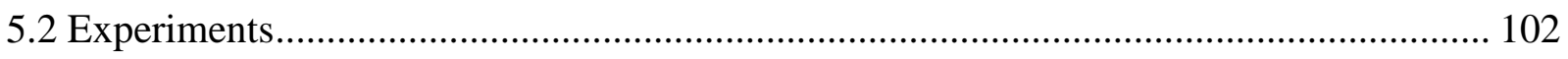

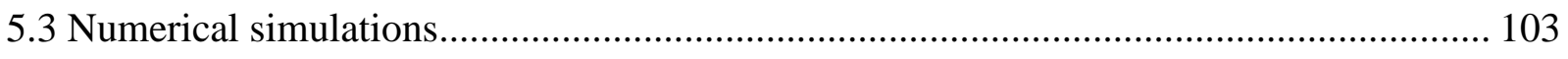

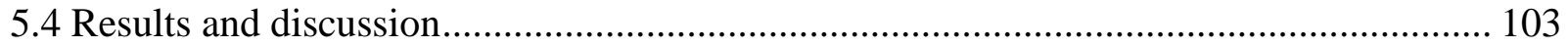

5.4.1 Further verification of numerical model for low impact angle and velocity............. 103

5.4.2 Relationship between average diameter of particles after impact and number of

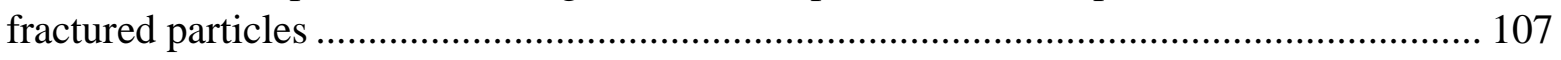

5.4.3 Influence of impact velocity and threshold for particle fracture ............................ 108

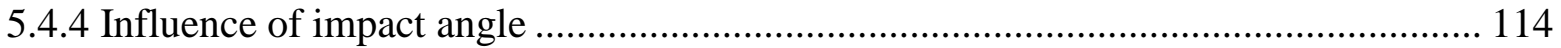

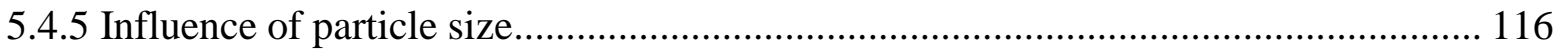

5.4.6 Influence of kinetic energy transfers perpendicular to surface on particle fracture ... 125

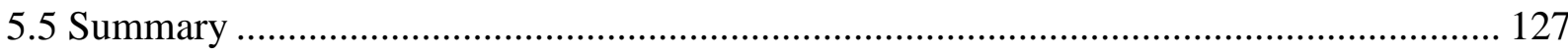

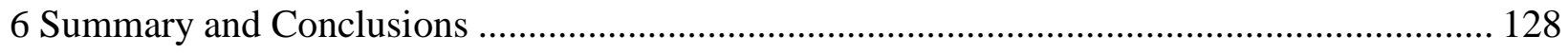

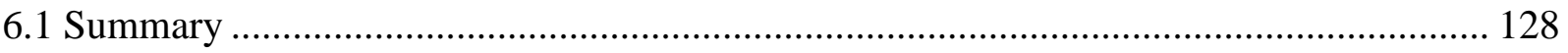

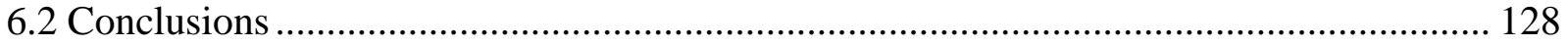

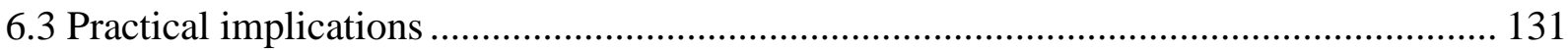

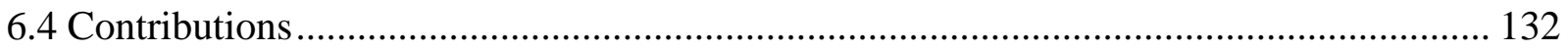

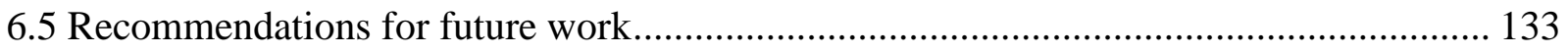

Appendix A Particle Size and Shape Distributions ..................................................... 135

Appendix B Modeling of Instantaneous Drag Force …................................................... 137

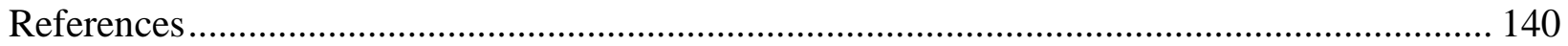




\section{List of Tables}

Table 2.1 Combination of process parameters used in the shadowgraphy and embedding measurements. Each condition was used for all four particles sizes described in Appendix A. .. 22 Table 2.2 Average particle linear velocity $(\mathrm{m} / \mathrm{s})$ of particles at different standoffs and jet pressures.

Table 3.1 Process parameters for embedding experiments and numerical simulations. 47

Table 3.2 Properties of Al $6061 \mathrm{~T} 6$ target and SiC abrasive particles. 54

Table 3.3 Model predicted (100 incident particles) percentage of the embedded particles using different friction coefficients, and corresponding measured value. Experimental conditions: grit $90 \mathrm{SiC}$ abrasives, perpendicular impact, $P=300 \mathrm{kPa}$, impact angle $=90^{\circ}, \mathrm{V}=120 \mathrm{~m} / \mathrm{s}$. 62

Table 4.1 Material constants for SiC ceramic [156] 87

Table 5.1 Process parameters using in shadowgraphy experiments. The last column indicates whether numerical models were also developed for a particular set of parameters. *Data in last row from Chapter 4 . 102

Table 5.2 Distribution of various characterizing parameters for the utilized SiC particles [99]. 103

Table A.1 Distribution of various characterizing parameters for the utilized SiC particles...... 135

Table B.1 Value of fit parameters used in eqs. B-2, B-3 and B-4 [89]................................. 138

Table B.2 Variations of Drag coefficient versus traveled distance for 3 sample particles....... 139 


\section{List of Figures}

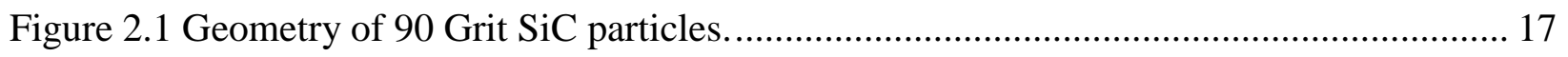

Figure 2.2 Distribution of: (a) in-plane area; (b) circular diameter; (c) roundness; (d) average thickness; (e) aspect ratio for 90 grit $\mathrm{SiC}$ abrasive. ………...................................................... 20

Figure 2.3 Shadowgraphy set up for measurement of particles velocity and orientation............. 21

Figure 2.4 Definition of (a) alignment angle $\varphi$, and (b) orientation angle $\theta$ for a typical particle.

Figure 2.5 SEM - showing embedded SiC particles (solid lines) and impact craters without

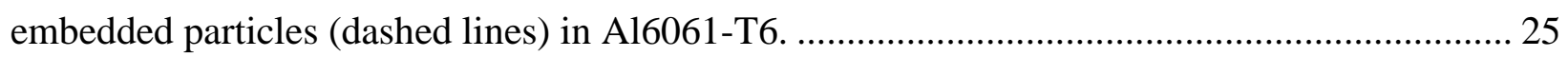

Figure 2.6 Schematic defining particle parameters used in model............................................ 28

Figure 2.7 Predicted and measured average particle linear velocities for grit 60 and 90 particles at $300 \mathrm{kPa}$. 30

Figure 2.8 Distribution of angular displacements of grit 60 particles at $300 \mathrm{kPa}$ at the following standoffs (a) nozzle exit; (b) $20 \mathrm{~mm}$; (c) $40 \mathrm{~mm}$. The average linear distance travelled by the

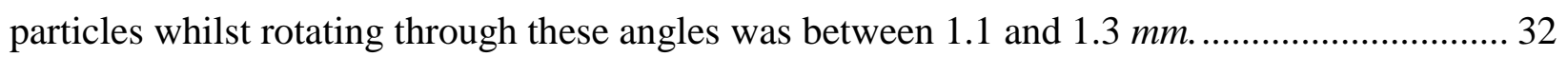
Figure 2.9 Variation of the alignment angle with aspect ratio at $P=300 \mathrm{kPa}$ and $20 \mathrm{~mm}$ standoff for (a): grit 90; (b): grit 60 particles. Scatter bars show the standard deviation.

Figure 2.10 Distribution of orientation angle at the standoff of $20 \mathrm{~mm}$ for grit $90 \mathrm{SiC}$ abrasives, Scatter bars show the standard deviation. 34

Figure 2.11 Average percentage of oriented particles for different particle sizes at different standoffs. Each bar shows the average of all tested pressures, and scatter bars show the standard deviation. 35

Figure 2.12 Predicted and measured distribution of particle orientation angles at nozzle exit and $40 \mathrm{~mm}$ standoff for (a) grit 90, and (b) grit 60 particles at $300 \mathrm{kPa}$. 36

Figure 2.13 Predicted and measured percentages of oriented particles $\left(0^{\circ}-10^{\circ}\right)$ at different standoffs for (a) grit 90, and (b) grit 60 particles at $300 \mathrm{kPa}$. Scatter bars on predicted values show standard deviation of multiple runs of the model using 200 particles. 37

Figure 2.14 Percentage of embedded particles at different standoffs for different particles sizes at $300 \mathrm{kPa}$. Scatter bars show the standard deviation. 38

Figure 2.15 Correlation between percentage of embedded and oriented particles at $300 \mathrm{kPa}$. The dashed line indicates a linear fit to all data. Scatter bars show standard deviations. 39 
Figure 2.16 Comparison of percentage of oriented particles and embedded particles for the tests at $10 \mathrm{~mm}$ standoff and $500 \mathrm{kPa}$ pressure with $40 \mathrm{~mm}$ standoff and $300 \mathrm{kPa}$ (grit 90). In both cases, the average particle velocity was $\sim 135 \mathrm{~m} / \mathrm{s}$. 40

Figure 3.1 Images of typical craters with (dashed lines) and without (solid lines) embedded SiC particles using (a) SEM; (b) EDS with white indicating presence of Si.................................. 49

Figure 3.2 Orientation angle $(\theta)$ of an abrasive particle in the jet. 50

Figure 3.3 Distribution of orientation angles at a $20 \mathrm{~mm}$ standoff distance from the nozzle. Grit 90 particles at $300 \mathrm{kPa}$ jet pressure [99]. The lines are only to guide the eye...... 51 Figure 3.4 Geometry of a random sample of: (a) modeled particles and (b) actual SiC particles.

Figure 3.5 3D view of atypical single particle impact model after embedment. 59 Figure 3.6 Variations of particle center of mass velocity vs impact time for different values of friction coefficient (0-2.5) for a typical grit 90 particle, $\theta=5^{\circ}$, Impact angle $=90^{\circ}, P=300 \mathrm{kPa}$, $V=120 \mathrm{~m} / \mathrm{s}$.

Figure 3.7 Variations of resultant force from SPH target to (a) an embedded particle, $\mu=1.5$; (b) a rebounding particle, $\mu=0.75$.

Figure 3.8 Comparison of predicted and measured percentage of particle embedment at various impact angles and two velocities, for grit 90 particles. Scatter bars show the standard deviation of 3 repeated experiments. 100 total impacts modeled.

Figure 3.9 Comparison of predicted and measured percentage of particle embedment at $V=87 \mathrm{~m} / \mathrm{s}$ (grit 60, standoff $=20 \mathrm{~mm}, P=300 \mathrm{kPa}$ ). Scatter bars show the standard deviation of 3 repeated experiments. 100 total impacts modeled.

Figure 3.10 Comparison of percentage of embedded grit 60 particles at various velocities at 40 $m m$ standoff and $90^{\circ}$ impact angle. 66

Figure 3.11 Impact and rotation of identically-shaped particles at $87 \mathrm{~m} / \mathrm{s}$ velocity and $90^{\circ}$ impact angle for (a) grit 60 (embedment), and (b) grit 90 (rebounded). 68 Figure 3.12 Resultant displacement of particle center of mass versus time for the two particles of Fig. 3.11. 69 Figure 3.13 Forces acting on an angular particle in contact along both faces [16]. $F_{\text {normal-elastic }}{ }^{L}$ and $F_{\text {normal-elastic }}{ }^{R}$ are the elastic normal forces and $F_{\text {tangential-elastic }}{ }^{L}$ and $F_{\text {tangential-elastic }}{ }^{R}$ are the tangential forces acting on a particle upon rebound. $A$ is the angularity and $\theta$ orientation of particles....... 70 
Figure 3.14 Distribution of critical value of friction coefficient as predicted by Getu et al. [16] for equivalent ideal rhomboid particles. All incident particles considered. ...................................... 71 Figure 3.15 Distribution of critical value of friction coefficient for modeled embedded particles

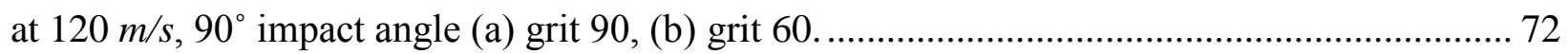

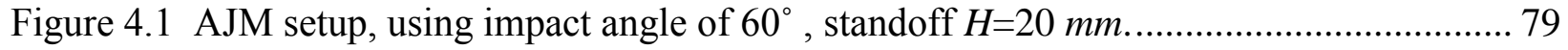
Figure 4.2 Experimental set up used for shadowgraphy experiments: (a) Shadowgraphic measurement system; (b) inclined target inside test chamber. 80 Figure 4.3 Typical shadowgraphy image showing the fracture and fragmentation of two typical particles impacting at a $60^{\circ}$ incidence angle and $100.3 \mathrm{~m} / \mathrm{s}$ : (a) before impact (b) after impact. 81 Figure 4.4 Particle geometry generation. (a) shadowgraphic image of a particle in flight (b) skeleton point representation (c) planar area (d) generated volume (e) meshed FE representation

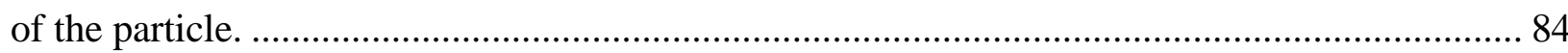
Figure 4.5 Contour plot of maximum principle strain for an impacting particle at (a) $t=0.1 \mu \mathrm{s}$ (b) $t=0.2 \mu s$

Figure 4.6 Crack initiation and propagation and fracture of particles at $V=120 \mathrm{~m} / \mathrm{s}$, impact angle= $60^{\circ}$ (a) $t=0 \quad$ (b) $t=0.1 \mu \mathrm{s} \quad$ (c) $t=0.15 \mu \mathrm{s} \quad$ (d) $t=0.5 \mu \mathrm{s}$ 90

Figure 4.7 Changes in the magnitude of sliding, kinetic, internal and total energy during the impact and fracture simulation for a typical grit 60 particle at $100 \mathrm{~m} / \mathrm{s}$ incident velocity and $60^{\circ}$ impact angle. 92

Figure 4.8 Distribution of particle circular diameter (a) before blasting, measured using microscope (average $=363 \mu \mathrm{m}$ ) and shadowgraphy (average $=307 \mu \mathrm{m}$ ), and (b) after impact, measured using microscope (average $=156 \mu \mathrm{m}$ ), predicted from numerical simulation (average $=$ $133 \mu \mathrm{m}$ ) and modified prediction to account for measurement bias (average $D_{\text {Circular }}=157.5 \mu \mathrm{m}$ ). 94

Figure 4.9 Distribution of particle roundness: Intact particles (average $=0.6$ ), predicted (average $=0.54$ ), and measured after blasting (average $=0.59$ ). .95 Figure 4.10 Typical images showing the impact and fragmentation of a particle (a) shadowgraphy image before impact, (b) shadowgraphy image after impact (c) modeled particle before impact and (d) modeled particle after impact. .96 
Figure 4.11 Distribution of (a) measured abrasive impact velocity (average $=100.3 \mathrm{~m} / \mathrm{s}$ ) (b) measured abrasive rebound velocity (average: $34.1 \mathrm{~m} / \mathrm{s}$ ) and predicted rebound velocity (average $36.1 \mathrm{~m} / \mathrm{s})$

Figure 5.1 Predicted (average $=317 \mu \mathrm{m}$ ) and measured (average $=323 \mu \mathrm{m}$ ) size distributions of grit 60 particles after impact at $46 \mathrm{~m} / \mathrm{s}$ and a $30^{\circ}$ impact angle, compared to before impact (average $=363 \mu \mathrm{m})$. 105

Figure 5.2 Predicted (average $=0.56$ ) and measured (average $=0.58$ ) roundness distributions of grit 60 particles after impact at $46 \mathrm{~m} / \mathrm{s}$ and a $30^{\circ}$ impact angle, compared to before impact (average= $0.6)$. 105

Figure 5.3 Distribution of predicted and measured velocity components for grit 60 particles at $V x=46 \mathrm{~m} / \mathrm{s}$ incident velocity and $30^{\circ}$ impact angle. 106 Figure 5.4 Relationship between measured normalized particle size after impact and predicted percentage of launched particles that fractured. $D f$ and $D i$ indicate measured average particle diameters before and after impact, respectively. 107 Figure 5.5 Measured size distributions of grit 60 particles before (average $=363 \mu \mathrm{m}$ ), and after impact for: (a) $30^{\circ}$ impact angle at $46 \mathrm{~m} / \mathrm{s}$ (average $=323 \mu \mathrm{m}$ ) and $100 \mathrm{~m} / \mathrm{s}$ (average $=211 \mu \mathrm{m}$ ); (b) $60^{\circ}$ impact angle at $46 \mathrm{~m} / \mathrm{s} \quad($ average $=309 \mu \mathrm{m})$ and $100 \mathrm{~m} / \mathrm{s}($ average $=156 \mu \mathrm{m})$. 109 Figure 5.6 Typical grit 60 particle at $30^{\circ}$ impact angle (a) before impact (b) $7.5 \mu \mathrm{s}$ after impact at $46 \mathrm{~m} / \mathrm{s} \quad$ (c) $7.5 \mu \mathrm{s}$ after impact at $100.3 \mathrm{~m} / \mathrm{s}$. 110

Figure 5.7 Measured roundness distributions of grit 60 particles before (average $=0.6$ ), and after impact for: (a) $30^{\circ}$ impact angle at $46 \mathrm{~m} / \mathrm{s}$ (average $\left.=0.58\right)$ and $100 \mathrm{~m} / \mathrm{s}$ (average=0.6); (b) $60^{\circ}$ impact angle at $46 \mathrm{~m} / \mathrm{s}$ (average $=0.6)$ and $100 \mathrm{~m} / \mathrm{s}($ average $=0.59)$. 111 Figure 5.8 Measured rebound angle distribution of grit 60 particles after impact at 46 and 100.3 $\mathrm{m} / \mathrm{s}$ for (a) $30^{\circ}$ impact angle (averages: $27^{\circ}$ for $46 \mathrm{~m} / \mathrm{s}$ and $100 \mathrm{~m} / \mathrm{s}$ ) (b) $60^{\circ}$ impact angle (averages: $29.5^{\circ}$ and $31^{\circ}$ for $46 \mathrm{~m} / \mathrm{s}$ and $100 \mathrm{~m} / \mathrm{s}$, respectively).

Figure 5.9 Measured velocity ratio distribution for grit 60 particles at $46 \mathrm{~m} / \mathrm{s}$ and $100 \mathrm{~m} / \mathrm{s}$ incident velocity at (a) $30^{\circ}$ impact angle (average: 0.51 and 0.48 for 46 and $100 \mathrm{~m} / \mathrm{s}$, respectively) (b) $60^{\circ}$ impact angle (average: 0.29 and 0.34 for 46 and $100 \mathrm{~m} / \mathrm{s}$, respectively) 114 Figure 5.10 Typical grit 60 particle at $100 \mathrm{~m} / \mathrm{s}$ incident velocity (a) $7.5 \mu \mathrm{s}$ after impact at $30^{\circ}$ impact angle (b) $7.5 \mu \mathrm{s}$ after impact at $60^{\circ}$ impact angle. 115 
Figure 5.11 Scatter of $V x$-Vy components of rebound velocity for grit 60 particles at $46 \mathrm{~m} / \mathrm{s}$ incident velocity and $30^{\circ}$ and $60^{\circ}$ impact angle. Lines indicate position of target surface........ 116 Figure 5.12 Predicted $($ average $=97 \mu \mathrm{m})$ and measured $($ average $=94 \mu \mathrm{m})$ size distributions of grit 120 particles after impact at $100 \mathrm{~m} / \mathrm{s}$ and a $60^{\circ}$ impact angle, compared to before impact $($ average $=152 \mu \mathrm{m})$.

Figure 5.13 Predicted (average $=0.57$ ) and measured (average $=0.57$ ) roundness distributions of grit 120 particles after impact at $100 \mathrm{~m} / \mathrm{s}$ and a $60^{\circ}$ impact angle, compared to before impact (average $=0.58)$. 118

Figure 5.14 Comparison between the fracture of typical modeled particles of different sizes at 100 $\mathrm{m} / \mathrm{s}$ incident velocity $60^{\circ}$ impact angle (a) particle \#1, grit 60 ; (b) particle \#1, grit 120 ; (c) particle \#2, grit 60 (d) particle \#2, grit 120 . 119 Figure 5.15 SEM images of particles before and after impact at $100 \mathrm{~m} / \mathrm{s}$ and $60^{\circ}$ impact angle for (a) grit 60 before impact (b) grit 60 after impact (c) grit 120 before impact (d) grit 120 after impact. 121

Figure 5.16 Distribution of measured and predicted values of $V x-V y$ on rebound at $100 \mathrm{~m} / \mathrm{s}$ incident velocity and $60^{\circ}$ impact angle (a) for grit 60 and (b) for grit 120 abrasives 122 Figure 5.17 Distribution of the measured velocity ratio for grit 120 and 60 particles at $100 \mathrm{~m} / \mathrm{s}$ incident velocity and $60^{\circ}$ impact angle. 123 Figure 5.18 Distribution of the measured rebound angle for grit 60 (average $31^{\circ}$ ) and grit 120 (average $30.5^{\circ}$ ) particles at $100 \mathrm{~m} / \mathrm{s}$ incident velocity and $60^{\circ}$ impact angle. 124 Figure 5.19 Contour plot of maximum principle strain for an impacting particle modeled in different sizes, after $t=0.25 \mu \mathrm{s}$ from impact at $60^{\circ}$ angle (a) particle \#2, grit $60,27 \mathrm{~m} / \mathrm{s}$ incident velocity ; (b) particle \#2, grit 120, $100 \mathrm{~m} / \mathrm{s}$ incident velocity. 125 Figure 5.20 Dependence on number of particle fragments per launched particle on normal kinetic energy for grit $60 \mathrm{SiC}$ abrasives under different operating conditions. The dotted line is a least squares linear fit. 126 Figure B.1 Two forms of simplified non-spherical shapes utilized in this study: (a) ellipsoid 1 and (b) ellipsoid 2 [89]. 138 


\section{List of Appendices}

Appendix A Particle Size and Shape Distributions .......................................................... 135

Appendix B Modeling of Instantaneous Drag Force …...................................................... 137 


\title{
Nomenclature
}

\author{
A \\ Angularity (degrees) \\ $\bar{A}$ \\ AR \\ Area \\ $A_{\text {particle }}$ \\ B \\ BHN \\ $\bar{B}$ \\ $c$ \\ C \\ $\bar{C}$ \\ $C_{D}$ \\ $C_{p}$ \\ D \\ $\bar{D}$ \\ $D_{1-} D_{5}$ \\ $\bar{D}_{1}, \bar{D}_{2}$ \\ $D_{\text {Circular }}$ \\ Df \\ Di \\ E \\ $E_{0}$ \\ $f$ \\ F \\ $F_{n}$ \\ G \\ h \\ H \\ Normalized strength parameter for undamaged material in Eq. 4.5 \\ The ratio of longest to shortest feret \\ In-plane area of particle $\left(\mu m^{2}\right)$ \\ Instantaneous projected frontal area of the particle $\left(\mu m^{2}\right)$ \\ Johnson-Cook constant in Eq. 3.6 ( $\mathrm{MPa}$ ) \\ Brinell hardness number \\ Normalized strength parameter for damaged material in Eq.4.6 \\ Johnson-Cook constant in Eq. 3.6 \\ Bulk Speed of Sound $(\mathrm{m} / \mathrm{s})$ \\ Strength parameter for strain rate dependence in Eq. 4.5 and 4.6 \\ Drag coefficient, \\ Specific heat $(\mathrm{J} / \mathrm{kg} \mathrm{K})$ \\ Cowper-Symonds constant in Eq. 3.9 \\ Damage factor, Eq. 4.9 \\ Johnson-Cook damage model constants in Eq. 3.11 \\ Parameter for plastic strain to cause damage in Eq. 4.10 \\ Equivalent circular diameter $(\mu m)$ \\ Measured average particle diameters after impact $(\mu \mathrm{m})$ \\ Measured average particle diameters before impact $(\mu \mathrm{m})$ \\ Modulus of elasticity ( $G P a)$ \\ Internal energy $(K J)$ \\ Field function \\ Accumulated damage \\ Net drag force $(N)$ \\ Shear Modulus $(G P a)$ \\ Particle length $(\mu m)$ \\ Standoff distance $(\mathrm{mm})$
}




\begin{tabular}{|c|c|}
\hline hel & Hugoniot elastic limit \\
\hline$I$ & Moment of inertia $\left(\mathrm{m}^{4}\right)$ \\
\hline$K_{1}-K_{3}$ & Pressure coefficients in Eq. $4.7 \mathrm{a}$ \\
\hline $2 l$ & Radius of influence domain in Eq. 3.1 \\
\hline$L$ & Longest feret of particle $(\mu m)$ \\
\hline$m$ & Johnson-Cook thermal softening exponent in Eq. 3.6 \\
\hline$m_{j}$ & Mass of SPH particle j in Eq. 3.2 \\
\hline$M$ & Strength parameter for damaged material in Eq. 4.6 \\
\hline$n$ & Johnson-Cook constant in Eq. 3.6 \\
\hline$N$ & Strength parameter for undamaged material in Eq. 4.5 \\
\hline$p$ & Hydrostatic stress $(M P a)$ \\
\hline$P_{\text {hel }}$ & Equivalent pressure at Hugoniot elastic limit (hel) \\
\hline$P$ & Pressure $(M P a)$ \\
\hline Perimeter & Perimeter of an abrasive $(\mu m)$ \\
\hline$P^{*}$ & Maximum normalized hydrostatic pressure \\
\hline$q$ & Cowper-Symonds constant in Eq. 3.9 \\
\hline$R$ & Roundness \\
\hline$R e$ & Reynold's number \\
\hline$S_{1}-S_{3}$ & Mie-Gruneisen constants in Eq. 3.12 \\
\hline$t$ & Time $(s)$ \\
\hline$T$ & Temperature $(K)$ \\
\hline$T_{\text {ins }}$ & Instantaneous temperature $(K)$ \\
\hline$T_{m}$ & Melting temperature $(K)$ \\
\hline$T_{\text {ref }}$ & Reference temperature $(K)$ \\
\hline $\bar{T}$ & Maximum tensile strength $(M P a)$ \\
\hline$T^{*}$ & Normalized temperature \\
\hline $\bar{T}^{*}$ & Maximum normalized tensile strength \\
\hline$v_{i j}$ & Relative velocity vector between SPH particle $i$ and $j$ in Eq. 3.3 and 3.5 \\
\hline$V_{\text {air }}$ & Air velocity $(\mathrm{m} / \mathrm{s})$ \\
\hline$V_{S}$ & Shock wave velocity $(\mathrm{m} / \mathrm{s})$ \\
\hline
\end{tabular}




\begin{tabular}{|c|c|}
\hline$V_{\text {particle }}$ & Particle velocity $(\mathrm{m} / \mathrm{s})$ \\
\hline$V x, V y$ & Components of rebound velocity $(\mathrm{m} / \mathrm{s})$ \\
\hline$W$ & Smoothing kernel function in Eq. 3.1 \\
\hline$x^{\prime}$ & Location of all the points inside the influence domain in Eq. 3.1 \\
\hline$x_{i}$ & Coordinate of SPH particle $i$ in Eq. 3.1 \\
\hline$X, Y$ & Coordinates of the Skelton points on the perimeter of the abrasive \\
\hline$X_{c p}$ & Distance from the center of mass to the center of pressure of the abrasive \\
\hline$\alpha$ & Rotation angle of a particle in flight $(\mathrm{rad})$ \\
\hline $\bar{\alpha}$ & Volume correction factor \\
\hline$\dot{\alpha}(t)$ & Particle angular velocity $\left(\frac{\mathrm{rad}}{\mathrm{s}}\right)$ \\
\hline$\ddot{\alpha}(t)$ & Particle angular acceleration $\left(\frac{\mathrm{rad}}{\mathrm{s}^{2}}\right)$ \\
\hline$\beta$ & Fraction of elastic energy loss converted to hydrostatic energy \\
\hline$\varepsilon_{e f f}^{p}$ & Effective plastic strain \\
\hline$\dot{\varepsilon}_{e f f}^{p}$ & Effective plastic strain rate \\
\hline$\dot{\varepsilon}_{e f f}$ & Effective strain rate \\
\hline$\dot{\varepsilon}^{*}$ & Normalized strain rate \\
\hline$\dot{\varepsilon}_{0}$ & Reference strain rate \\
\hline$\varepsilon_{f}^{p}$ & Plastic failure strain \\
\hline$\Delta \varepsilon^{p}$ & Increase in plastic strain \\
\hline$\varepsilon^{f}$ & Failure strain \\
\hline$\Omega$ & Domain of integration in Eq. 3.1 \\
\hline$\gamma$ & Gruneisen Constant \\
\hline$\mu$ & Friction coefficient \\
\hline$\mu_{c}$ & Critical friction coefficient \\
\hline$\mu_{\text {hel }}$ & Constant in Eq. 4.2 and 4.3 \\
\hline $\bar{\mu}$ & Rate of change in density \\
\hline$v$ & Poisson Ratio \\
\hline$\theta$ & Orientation angle (degrees) \\
\hline
\end{tabular}




$\begin{array}{cc}\rho & \text { Density }\left(\frac{\mathrm{kg}}{\mathrm{m}^{3}}\right) \\ \rho_{0} & \text { Initial density }\left(\frac{\mathrm{kg}}{\mathrm{m}^{3}}\right) \\ \rho_{\text {air }} & \text { Air density }\left(\frac{\mathrm{kg}}{\mathrm{m}^{3}}\right) \\ \rho_{j} & \text { Density of SPH particle } j \text { in Eq. 3.2 } \\ \sigma_{0}, & \text { Static yield stress in Eq. 3.6 }(\mathrm{MPa}) \\ \sigma_{e f f} & \text { Effective stress }(\mathrm{MPa}) \\ \sigma_{\text {hel }} & \text { Component of stress tensor for SPH particle } j \text { in Eq. 3.4 }-3.5 \\ \sigma_{j} & \text { Uniaxial yield strength }(\mathrm{MPa}) \\ \sigma_{S t a t i c} & \text { Instantaneous yield stress }(\mathrm{MPa}) \\ \sigma_{y} & \text { Normalized strength of the intact material } \\ \sigma_{i}^{*} & \text { Normalized strength of the damaged material } \\ \sigma_{f}^{*} & \text { Alignment angle }(\text { degrees }) \\ \varphi & \text { Torque }(\mathrm{N} . \mathrm{m})\end{array}$




\section{Introduction}

\subsection{The role of particle embedment and fracture in solid particle erosion}

Solid particle erosion is a complicated phenomenon involving the repeated impingement between the abrasives and a surface that leads to deformation and erosion of target substrate [1-3]. Solid particle erosion is utilized constructively in abrasive jet machining (AJM), abrasive water jet machining (AWJM) and in blast stripping. In such constructive applications, small abrasive particles are accelerated by a pressurized fluid and the resulting jet is directed toward the target substrate through the nozzle exit. Abrasive jet micro-machining (AJM), is a common example of solid particle erosion applications, used to machine various features such as micro-channels and holes, optoelectronic components and micro-electro-mechanical-systems (MEMS) [4]. Destructive applications of solid particle erosion concern the unwanted impact of particles with the surface of mechanical components, such as turbine blade or helicopter propeller.

Depending on the variations of erosion rate (ER) versus impact angle, two erosion modes are often distinguished in the literature: brittle and ductile erosion [1,5,6,7]. Erosion on brittle materials generally involves fracture and crack propagation, while ductile materials are usually eroded through cutting, ploughing and chip separation mechanisms [8]. For brittle materials the maximum erosion rate occurs at perpendicular impact while for ductile materials, it happens at shallow impact angles [9].

One of the undesirable features of constructive solid particle erosion of ductile materials is particle embedment, i.e. the tendency for some particles to not rebound but instead remain buried in the material. Particle embedding can be a problem in a wide variety of applications because embedded abrasives may reduce the erosion rate [10], and for AJM in particular, negatively impact the heat transfer rate in abrasive jet micro-machined heat exchangers, and increase the roughness, thus affecting the flow in micro-machined microfluidic devices [11,12]. However, embedment of particles may also be considered as a beneficial phenomenon when more resistance against erosion is required $[13,14]$. Experimental investigations $[6,15]$ show that in many practical erosion testing and abrasive jet machining scenarios, some of the abrasive particles break upon impact, and this may influence the erosion rate and mechanism.

To the knowledge of author, fracture of abrasives has not been modeled before. Studying the fracture of the particles can help to explain the reason of the breakage of the abrasives and its 
influence on the extent of the embedment and also erosion rate. As will be seen in Section 1.2, the existing purely theoretical models of embedment are valid only under a limited set of process parameters such as fully spherical or rhomboid particles and could not be applied for a strain rate sensitive or strain hardening material. Besides, no practical numerical particle fracture model capable of predicting the fracture of realistic abrasives, geometry and rebound kinematics of fragments was introduced in literature; and the only theoretical model could not be applied in practical applications.

\subsection{Literature review}

In this section, a brief literature review is presented to facilitate a concise overview of the previous investigations about particle embedment of angular and spherical particles and for the fracture of abrasives upon impact. It is intended to illustrate the areas where further research is required. More extensive literature reviews on specific topics are provided at the beginning of Chapters 2-5.

\subsubsection{Particle embedment in erosive processes}

Determining the process conditions that result in particle embedment is a critically important to avoid undesirable consequences in erosion testing or abrasive jet machining applications. Getu and co-workers [16] were the first to rigorously study the required conditions that lead to embedment of spherical and angular abrasives. Although their focus was on embedding into polymers, many of the basic concepts can also be applied for metals as well, since erosive behaviour of both is categorized as ductile erosion.

\subsubsection{Embedment of angular particles}

Getu et al. [16] identified two criteria for particle embedment in solid particle erosion processes: The first criterion was that the particle trajectory during impact would be such that a contiguous surface contact between the target and abrasive would be maintained during the impact, and the second was that the friction force that tended to keep the particle embedded would be larger than the elastic rebound force. Getu et al. [16] defined a critical value of static friction 
coefficient between the target and impacting particle which depended on the angularity of the rhomboid particles that they had modelled. They illustrated that the particle would embed if the realistic static friction coefficient is greater than the critical value. They reported that the critical magnitude of friction coefficient for highly angular particles was relatively small and consequently, highly angular particles are more likely to embed in comparison to the particles that are blockier in shape. This is because, although the direction of the elastic forces on the particles may change as a function of the crater shapes, incident angles, impact velocities and abrasives orientations, assuming that the first condition is satisfied, the ratio of tangential to normal elastic force merely depends on the angularity of the particle (i.e. its sharpness). It can be concluded that for sharper particles with higher angularity, the above mentioned force ratio is smaller and thus such a particle is more likely to embed in the target.

With respect to the aforementioned circumstances it was inferred that if contiguous contact was maintained during impact, then it would be possible to assess the propensity for embedding of each individual abrasive at various incident angles with an acceptable accuracy, provided that the static friction coefficient between the abrasives and target surface could be determined. Getu et al. used a simplified perfectly plastic model to determine the trajectory of idealized rhomboid particles as they impacted, and thus whether contiguous contact was maintained. They also reported that the static friction coefficient for a typical system of particles and target would be approximately the same and independent from abrasives size and incident angle [16]. Getu et al. found that particles do not embed if the impact velocity is smaller than a threshold value, and figured that there was a preferred orientation for each particle that results in maximum likelihood of embedment. Getu et al. [16] observed that particle embedment could occur under the minimum incident velocity, if major axis and velocity vector of particle are aligned. The model of Getu et al. [16] was certainly pioneering, however it suffers from some limitations. Most significantly, it cannot be extended to predict the embedment of abrasives with more realistic angular shapes into a strain rate sensitive material or into a material for which strain hardening is significant; these shortcomings will be addressed in Chapter 3 of this thesis.

\subsubsection{Embedment of spherical particles}

Getu et al. [16] studied the embedment of spherical particles and hypothesized that 
embedment of a spherical abrasive would occur under the condition that the penetration depth into the target was more than its radius. In this case, displaced material would surround the particle, and prevent the elastic rebound forces from ejecting the particle. Walley and Field [15] also studied the embedment of spherical particles and reported that for impact under adequately high impact velocity, displaced material will surround the abrasive and prevent its rebound. As discussed in Section 1.2.1.1, static coefficient of friction is an important key factor that determines the embedding of angular particles. However, for spherical particles, it is the dynamic coefficient of friction that determines the maximum penetration depth and thus determines their embedment.

\subsubsection{Process parameters that affect particle embedment}

There have been numerous experimental works aimed at determining the parameters that affect particle embedment. Particle incident velocity is an important parameters affecting the particle embedding; and impacting particles also embed into a target even at low incident velocities. For example, Lathabai et al. [17] used SEM and EDX analysis and detected $\mathrm{SiO}_{2}$ abrasives embedded in a flame-sprayed Nylon coating blasted at velocities as low as $3.5 \mathrm{~m} / \mathrm{s}$. Generally, embedment will not occur if the incident velocity is below a threshold value; in other words, a particle can theoretically embed into the target if it contains enough kinetic energy [16].

Temperature is an important factor that affects the mechanical properties of various materials (e.g., polymers) [18-20], and therefore may also influence embedding. Getu et al. [21] conducted extensive investigations on the erosive behaviour of polymers using AJM, under cryogenic temperatures and found that the percentage of embedded particles was significantly reduced at cryogenic temperatures, for any combination of impact angle and velocities. They concluded that cryogenic conditions improves the efficiency of abrasive jet machining of polymeric materials such as ABS and PTFE that demonstrate a high extent of particle embedment at room temperature.

Incident angle is an important factor that affects the propensity for particle embedment. For example, it has been reported that the percentage of embedded particles decreases as a shallower impact angle is utilized [16]. Wu et al. [22] eroded different types of 2014 aluminium alloy using $\mathrm{SiC}$ and $\mathrm{Al}_{2} \mathrm{O}_{3}$ abrasives and reported that the percentage of particle embedment was higher at normal incident angle. Sheldon et al. [23] reported that hardness of the target surface also plays an important role in particle embedment. They studied the embedment of abrasives in applications 
that concern the repeated impact of particles and reported a lower percentage of embedded particles due to the presence of a work hardened layer [23]. The influence of impact angle and incident velocity on the likelihood of particle embedment will be covered in Chapter 3.

\subsubsection{Quantification of embedded particles}

Quantification of embedded particles is an important aspect of such studies and is considered as challenging due to the complexities involved in distinguishing the embedded abrasives from the neighbouring surface material. Getu et al. $[16,21]$ used scanning electron microscopy (SEM) and energy dispersive X-ray spectroscopy (EDX) extensively to evaluate the proportion of total surface area of the polymeric materials covered by embedded $\mathrm{Al}_{2} \mathrm{O}_{3}$ particles. Amada et al. [24] examined grit-blasted steel utilizing an electron probe micro-analyser (EPMA) and image analysis, and reported $10 \%$ of area coverage by $\mathrm{Al}_{2} \mathrm{O}_{3}$ and $\mathrm{ZrO}_{2}$ particles. Momber et al. [25] studied the grit contamination on hot-rolled low-carbon steel utilizing SEM and image analysis and reported approx. $8 \%$ of area coverage by embedded $\mathrm{Al}_{2} \mathrm{O}_{3}$ particles.

\subsubsection{Removal of embedded particles}

Various practical methodologies have been proposed for the removal/detachment of embedded abrasives. Toscano and Ahmadi [26] established theoretical models for the removal of embedded abrasive by the impact of $\mathrm{CO}_{2}$ pellets, and found that sufficiently small $\mathrm{CO}_{2}$ pellets can remove the very small embedded particles. Getu et al. [27] applied various techniques to remove the embedded particles caused by AJM, including: post-blasting the target surface using other types of impacting abrasives that would themselves not embed, ultrasonic cleaning of the target

after AJM, pressing a PVC electrical insulation adhesive tape onto the sample surfaces, liquid nitrogen freezing method, and combinations of these techniques. The most effective was found to be the freezing method in which blasted samples were frozen by dipping them in liquid nitrogen $\left(\mathrm{LN}_{2}\right)$ after being dipped in distilled water, and then exposed to room temperature.

\subsubsection{Particle orientation in a flow}

As shown by Hutchings [28], and Papini and co-workers [29-32], the orientation of the 
particles at the moment of impact can strongly affect the resulting erosion mechanism. As mentioned in Section 1.2.1.1, Getu et al. [16] showed that particle orientation may also affect the likelihood of the particles remaining embedded into the target material. While the model of Getu et al. [16] predicted that certain particle orientations are more favorable to particle embedment, the particle orientations within an actual abrasive jet were not measured. These measurements are done in Chapter 2 of the present dissertation.

There are few existing analytical models capable of predicting the influence of the process parameters such as jet pressure and travel distance on the rotation of particles in an air jet. Behavior of spherical particles in a gas-solid flow, such as particle density and size, and the viscosity and regime of the flow on the behavior of the particles have been studied by investigators such as Marchioli et al. [33], Kuerten [34] and Kulick et al. [35]. Most actual applications, however, involve the use of irregularly shaped particles, rather than idealized spherical ones. Because of the forces and moments that act on non-spherical particles in fluid flows, their direction of motion can potentially be influenced by their orientation. Several investigators, including Jeffery [36], Brenner [37, 38] and Harper and Chang [39] studied the behavior of elongated ellipsoidal particles theoretically. Fan and Ahmadi [40], Zhang et al. [41] and Parsheh et al. [42] studied the behavior of non-spherical particles numerically and experimentally. Most of these investigations focused on Stokes flow in which large aspect ratio have been found to be most likely to align with the flow direction [43]. In their review of the literature on the behavior of non-spherical particles in high Reynold's number flows, Mando and Rosendahl [44] noted that depending on the regime of fluid flow and the aspect ratio, the particles may experience a preferred orientation. Mortensen et al. [45], Paschkewitz et al. [46] and Zhang et al. [47] applied numerical simulation (DNS) to study the behavior of ellipsoidal particles in a turbulent flow and reported that the tendency for alignment increases with aspect ratio. The newly presented theoretical model in Chapter 2 is applicable to predict the instantaneous orientation of particles in an abrasive flow, typical of realistic AJM applications.

\subsubsection{Abrasive particle fracture due to impact}

Abrasive particles are generally relatively friable, and thus may fracture upon impact. As early as 1970, Tilly and Sage [48] reported that impacts between quartz particles at approximately 
$91 \mathrm{~m} / \mathrm{s}$ and metal, nylon and fiberglass target not only resulted in target material extrusion and plastic deformation, but also abrasive fracture. Particle fracture depends on different parameters such as the mechanical and geometrical characteristics of the target and particle, particles size and shape, hardness and toughness and also different operation parameters such as particle size, incident velocity, angle, etc. .

\subsubsection{Influence of particle size on particle fracture}

Salman et al. [49] conducted various single impact experiments using 3.14, 5.15 and 7.15 $m m$ spherical $\mathrm{Al}_{2} \mathrm{O}_{3}$ particles at incident velocities under $35 \mathrm{~m} / \mathrm{s}$ and reported that the larger abrasives were more likely to fracture. They attributed this to the larger flaws in the coarser abrasives which resulted in a lower fracture toughness. Akbarzadeh et al. [50] studied particles fracture at 90 and $130 \mathrm{~m} / \mathrm{s}$ incident velocity in different impact angles and reported a higher degree of fracture and fragmentation for the larger abrasives. Tilly and Sage [48] also studied the targetabrasive interaction and reported that larger particles were more susceptible to fracture.

\subsubsection{Influence of particle velocity on particle fracture}

Incident velocity has been found to be critically important to particle fracture in solid particle erosion applications. For example, Salman et al. [49] reported that fracture of particles may occur at velocities as low as $5 \mathrm{~m} / \mathrm{s}$ for $5.15 \mathrm{~mm}$ alumina spheres impacting a streel surface at normal incident angle. They also observed that a decrease in abrasive incident velocity led to fast transition from a full fracture to no fragmentation mode [49]. Such a transition was found to occur at a narrower range of velocity for larger particles than smaller ones. For example, smaller particles

fracture at a higher impact velocity compared to coarser ones, and the maximum incident velocity at which no particle fracture would be observed, is lower for the larger particles. Sparks and Hutchings [51] studied the fragmentation of 125-150 $\mu \mathrm{m}$ silica abrasives upon impact on silicate glass ceramic and reported that particle fracture was strongly proportional to incident velocity. Particle velocity reportedly also influences the fragment size and may also affect fragment shape [49]. Tilly and Sage [48] also figured that the increase in incident velocity has a direct influence on particle fracture and fragmentation. 


\subsubsection{Influence of incident angle on particle fracture}

Experimental observations show that number of unbroken particles increases at shallower incident angles at any typical velocity [49]. It was also reported that using a typical incident velocity, the number of unbroken abrasives at impact angles larger than $50^{\circ}$ was significantly lower than those of impact angles smaller than $50^{\circ}$, and that the critical value of velocity required for onset of particle fracture at smaller impact angles was higher. However, the transition from zero to total fragmentation of particles occurred much faster at higher impact angles in comparison to gradual trend observed at small impact angles [49]. Akbarzadeh et al. [50] figured that particles size would have a greater effect on particle fracture at a larger impact angle.

\subsubsection{Influence of surface hardness on particle fracture}

Surface hardness may change during impact due to the plastic deformation and generation of a work-hardened layer [52-54]. The work hardened layer formed by the impact of particles would be different while using large particles in comparison to small particles [50,55]. Misra and Finnie [52] and Akbarzadeh et al. [50] reported that due to the higher energy content, the coarser abrasives contribute to a higher degree of strain hardening which is one of the reasons for a greater degree of particle fracture for larger particles. Salman et al. [49] also reported that the number of unbroken particles at the same velocity was higher for softer targets.

\subsubsection{Modeling of particle fracture}

There have been some attempts to develop analytical models of particle fracture due to impact. Murugesh et al. [56] presented a theoretical model to predict the fracture of abrasives impacting an alumina target, based on the hypothesis of Lawn and Evans [57] with regards to the presence of a critical penetration depth to cause fracture of a brittle material. In their theoretical model, Muragesh et al. also assumed the existence acritical penetration depth of the target into the particle required for fracture of the particle [56]. The likelihood of particle fracture was found to depend on the ratio of the fracture toughnesses, hardnesses and Young's moduli of the abrasive and target, as well as the intrinsic flaw population of the two materials. Critical penetration depth Increased inversely with the extent of microstructure flaws in the target. This refers to the fact that crack initiation occurs more rapidly in presence of a larger number of flaws. This model, however is based on a simplified assumption of a penetrating target material into the impacting particle, and 
cannot predict the size and shape, rebound velocity and angle of fragments. These shortcomings will be addressed by introducing a numerical model of the impact process in Chapter 4 . The model will also allow the effect of many of the process parameters discussed in Chapter 3 on embedment to be assessed.

\subsubsection{Numerical simulation of particle fracture in solid particle erosion}

Several investigators tried to model cracking/fracture of brittle materials using numerical methods. Brittle and semi brittle materials experience micro-cracking in a localized narrow high strain zone under mechanical load [58]. Numerical simulation of the high strain narrow zone in fractured solids is still a challenging problem.

\subsubsection{Smooth particle hydrodynamics (SPH) versus element free Galerkin (EFG)}

Numerical techniques are powerful tools that help researchers achieve a better understanding about the damage and fracture phenomena. Grid based and mesh free methods have been applied for this type of modeling extensively by different researchers. In general, an unfavorable problem in many of the finite element $(\mathrm{FE})$ simulations of fracture is the distortion of element that results in excessive reduction of the time step that leads to extremely slow, and in some cases, termination of simulation [59].

SPH is a vastly used mesh free methods which has also been utilized for fracture modeling. There are, however, some inaccuracies such as tensile instability and rank deficiency associated with SPH modeling [60-63]. Hiermaier [64] reviewed the different types of formulations and possible solutions for the numerical instabilities. Reidel et al. [65] utilized SPH to model the fracture on $\mathrm{SiC}$ ceramic target under high velocity impact and observed that although SPH can simulate the void opening, the crack patterns were highly influenced by the smoothing functions.

Belytschko and his colleagues [66-68] has had a significant contribution in development of novel numerical techniques applicable for modeling of fracture in solids [66,67]. Element Free Galerkin (EFG) is a practical techniques they have presented [66-68]. Belytschko et al. [68] introduced the EFG to model static and dynamic fracture problems in the mid 1990's. This technique was later verified by simulation of the impact in various experiments [66]. The EFG method has since been used by a variety of researchers and shown to be a good candidate for 
simulation of cracking and fracture in brittle materials without the unfavorable re-meshing required in FE methods. For example, Morka [69] applied EFG to simulate the impact of a projectile on ceramic target supported by an aluminum plate in LS-Dyna and reported less than $20 \%$ error in comparison with experiments. Mesh free techniques in the context of EFG were also utilized to model cracking in ceramic piezoelectric parts under electro-mechanical loads [70]. Chapter 4 of the present thesis utilized EFG method to model particle fracture in realistic AJM applications.

\subsubsection{Constitutive equation for modeling brittle materials}

Choosing an appropriate material constitutive model is an essential part of fracture simulation. The Johnson-Holmquist ( $\mathrm{JH})$ constitutive model for ceramics is a powerful tool in describing the mechanical behavior of ceramic materials [71,72]. JH is capable of calculating the influence of fracture/damage of material on the remaining strength and consequent bulking which happens under compressive failure [73]. Quana et al. [74] utilized JH material model to simulate $\mathrm{SiC}$ part and validated the results through comparison with impact experiments. Guo et al. [75] applied JH in their model for cutting optical glass using SPH method and reported that material removal was in the form of breakage and fracture of the glass ceramic. Wang and Yang [76] studied the erosive behaviour of $\mathrm{SiC}$ brittle material using $\mathrm{JH}$ and reported a reasonable accuracy in comparison with experimental data from literature. Kaufman et al. [77] also applied JH to simulate the behaviour of Alumina ceramics under impact. Reidel et al. [65] used JH model to apply plasticity and failure to the $\mathrm{SiC}$ target. Lee and Yoo [78] also modeled the impact on a ceramic layer using JH model. JH material model was also utilized by Lundberg [79] to simulate the behaviour of different ceramics including $\mathrm{SiC}$ and alumina. In Chapter 4, the JH model will be for the first time applied to a numerical model of particle fracture that will lead to new insights regarding the mechanisms of particle fracture in solid particle erosion.

\subsubsection{Summary}

The preliminary survey on the embedment and fracture of abrasive particles in a solid particle erosion applications $[16,21,27,50]$ demonstrated that these two phenomena play an important role in the efficiency of the erosion mechanism. There are many different factors that influence the likelihood of particle embedment and/or fracture. Despite existing studies of 
embedment, some aspects of the embedment phenomenon remain poorly understood due to the complexity of the involved mechanisms. Existing embedment models such as that of Getu et al. [16] are pioneering, but at the same time they are highly simplified and the influence of, e.g., particle shape, strain rate, strain hardening, damage accumulation, and thermal softening of the material have not been directly considered. Similarly, the very few studies that focused on the fracture of abrasives were mostly experimental and no reliable numerical model capable to predict the fracture of abrasives has yet been developed. Finally, the few existing experimental studies in the realm of solid particle erosion have failed to report particle orientations within the abrasive jet and how they may affect embedment and fracture, and have failed to report accurate particle rebound kinematics. The objectives of the present thesis, address these shortcomings using stateof-the-art laser shadowgraphy methods, together with powerful numerical simulations in order to achieve a better understanding over the dominant embedding/fracture mechanisms and influential parameters.

\subsection{Objectives}

The main objective of this thesis is to identify the fundamental mechanisms of particle embedment and fracture, and to understand the effect that fundamental process parameters have on these two phenomena under conditions typical of solid particle erosion testing or abrasive jet machining applications. This main objective will be achieved by meeting the following secondary objectives:

(i) Measure and model the instantaneous size, shape, and orientations of particles in flight within an abrasive jet, and identify implications for particle embedding (Chapter 2).

(ii) Develop and experimentally verify a three-dimensional numerical model of the impact and possible embedment of realistically-shaped single angular particles on an aluminum alloy target. Use the model to understand the role of process parameters such as particle velocity, shape, etc. on the likelihood of embedment (Chapter 3).

(iii) Develop a three-dimensional numerical model to predict the fracture of realisticallyshaped single angular particles impacting an aluminum alloy target (Chapter 4).

(iv) Develop a novel double pulsed laser shadowgraphy technique in order to characterize the incident and fractured topography of particles before and after impact, and use the 
measurements to experimentally verify the model of (iii), and to understand the role of process parameters on the propensity for particle fracture (Chapter 5). 


\section{Measurements and modeling of instantaneous particle orientation within abrasive air jets and implications for particle embedding}

This chapter is based on the following published paper:

V. Hadavi, B. Michaelsen, M. Papini, Measurements and modeling of instantaneous particle orientation within abrasive air jets and implications for particle embedding, Wear 336-337 (2015) 9-20.

\subsection{Introduction}

Many industrial applications involve damage due to the impingement of a jet of fast moving solid particles propelled by a fluid flow. Solid particle erosion is a complex process involving the successive impact of abrasive particles on a target that results in material removal from the target surface [1-3]. Examples of such solid particle erosion processes include erosion in dust collectors, particle transportation in pipes and channels, and abrasive jet machining processes. Erosion on brittle materials generally involves fracture and crack propagation, while ductile materials are usually eroded through cutting, ploughing and chip separation mechanisms [80,81]. As shown by Hutchings [28], and Papini and co-workers [29-32], the orientation of the particles

at the moment of impact can strongly affect the resulting erosion mechanism. Getu et al. [16] have shown that particle orientation may also affect the likelihood of the particles remaining embedded into the target material.

Particle embedding can be undesirable in a variety of applications. For example, it may cloud the results of solid particle erosion testing of polymers and other soft materials since the embedded particles may shield the target surface from further impacts. In the abrasive jet micromachining (AJM) of polymer microfluidic chips, a similar mechanism reduces the etch rate [10], and also increases the surface roughness, thus affecting fluid flow [11,12]. In the AJM of microheat exchanger applications, the heat transfer rate may also be reduced due to the presence of embedded particles [82].

Getu et al. [16] identified two criteria for particle embedment in solid particle erosion 
processes: (i) that contiguous contact between the particle and target be maintained throughout the impact, and (ii) that the magnitude of the static friction forces reach a critical value. Both of these were hypothesized to strongly depend on particle orientation, i.e. angular particles were more likely to embed when their major axis connecting the leading vertex to the center of mass aligned with the velocity vector upon impact. While the model of Getu et al. [16] predicted that certain particle orientations are more favourable to particle embedment, the particle orientations within an actual abrasive jet were not measured. The present study is thus mainly motivated by the unanswered question of which process parameters lead to the orientations favourable for embedding.

There are few existing analytical models capable of predicting the influence of the process parameters such as jet pressure and travel distance on the rotation of particles in an air jet. Most studies have focused on the behavior of spherical particles in a gas-solid flow, which, due to their symmetry, are simpler to analyze and measure. In these cases, the effect of parameters such as particle density and size, and the viscosity and regime of the flow on the behavior of the particles have been studied by investigators such as Marchioli et al. (2007) [33], Kuerten (2006) [34] and Kulick et al. (1994) [35]. Most actual applications, however, involve the use of irregularly shaped particles, rather than idealized spherical ones.

A number of researchers have defined parameters to describe non-spherical particle shape, in order to assess their behavior in a fluid. For example, Wadell [83] introduced a sphericity factor, $\Phi$, defined as the ratio of the surface area of an equivalent sphere having the same volume as the actual particle, to the surface area of the actual particle. Hözler et al. [84] utilized two measures of sphericity, one in the lengthwise direction and the other in the crosswise direction, in order to relate the drag force to the orientation of particles travelling in a fluid. On the other hand, Loth et al. [85] suggested that the shape of a particle is best described by its aspect ratio.

Because of the forces and moments that act on non-spherical particles in fluid flows, their direction of motion can potentially be influenced by their orientation. Generally, the rotational motion of non-spherical particles and their likelihood of orientation with fluid flow depends on the shape of particle and the Reynolds number regime of the flow. The behavior of elongated ellipsoidal particles has been analytically studied by several investigators. Jeffery (1922) was one of the first [36], while Brenner (1963, 1964) [37,38] and Harper and Chang (1968) [39] also further developed the theoretical models. The behavior of non-spherical particles has also been studied 
numerically and experimentally by several investigators such as Fan and Ahmadi (1995) [40], Zhang et al. (2001) [41] and Parcheh et al. (2005) [42] during the past two decades. However, most of these investigations focused on Stokes flow in which case large aspect ratio have been found to be most likely to align with the flow direction [43]. Other Stokes flow studies such the one by Fan and Ahmadi [40] discuss the types of forces acting on a particle such as the shear induced lift. Studies in the realm of Stokes flow are useful in applications such as those describing blood flow or in the paper industry to analyze fiber flow, rather than the presently considered high speed turbulent abrasive jet flow.

Particles in a turbulent flow may exhibit quite complex behavior which is dramatically different from that in Stokes flow. In general, not only may the motion of particles be influenced by the turbulent flow, but also the characteristics of the flow may be altered by the motion of the particles. For example, depending on the flow regime and the shape of particles, non-spherical particles are often subject to an irregular or wobbling behavior in a turbulent flow [86]. This implies that the particle secondary motion may weaken the turbulent phase of the flow as a portion of their linear kinetic energy is transformed to particle rotational motion. Depending on the particle shape and size, the interaction between non-spherical particles and the flow can potentially intensify or weaken the turbulence.

Most models of the interaction between non-spherical particles and fluid flow focus on idealized disks, cylinders, long fibers and ellipsoids, for which a large variety of shapes can be described using few geometrical parameters. In their review of the literature on the behavior of non-spherical particles in high Reynold's number flows, Mando and Rosendahl [31] noted that flaky (as opposed to blocky) particles have been modeled using flat ellipsoids and disks of various aspect ratios. Depending on the regime of fluid flow and the aspect ratio, the particles may experience a preferred orientation [44]. For example, Christiansen and Barker [87] and Clift et al. [88] claim that particles above an aspect ratio of 1.7 result in significant secondary (i.e. rotational) motion. However, Zhang et al. numerically modeled the behavior of elongated ellipsoidal particles in a turbulent fluid flow, and found that only particles with an aspect ratio greater than 5 are likely to rotate and align with the flow direction [41]. Mortensen et al. [45] applied direct numerical simulation (DNS) to study the behavior of ellipsoidal particles in a turbulent flow and also reported that the tendency for alignment increases with aspect ratio. In another DNS model, Paschkewitz et al. [46] found that that rigid slender fibers are most likely to be aligned. They also calculated 
reductions in drag (up to 26\%) depending on the aspect ratio and reported that particle shape can significantly affect the turbulence. Finally, Zastawny et al. [89] used DNS to estimate the lift, drag forces and torques that act on four different non-spherical particles in a gas flow.

A major difficulty associated with modeling the secondary motion of non-spherical particles is the determination of an appropriate drag coefficient, which in general depends on both the particle shape and instantaneous alignment. While fit parameters have been used to derive drag coefficients [89], this has been done only for a limited range of particle geometries and flow regimes, and most studies ignore the influence of the instantaneous particle alignment relative to the flow direction.

Many techniques have been developed to measure particle behaviour in fluid-particle flows. The earliest experimental studies of the orientation of particles appear to have been conducted by monitoring macroscopic particles in viscous fluids (Okagawa and Mason [90]). Later on, Salem and Fuller [91] studied the behavior of particles using an optical technique that captured the two-dimensional distribution of the small angle light scattering of particles in a flow. Bernstein et al. [43] applied a coupled system of microscopic video-photography and image analysis to determine the orientation of cylindrical particles both in laminar and turbulent water flows. They reported that the orientation of cylindrical particles was influenced by the particle rotational diffusion coefficient and flow velocity gradient. For flows more typical of abrasive jets, Ruff and Ives [92] developed a rotating double disk apparatus that applied the time of flight principle to measure the average abrasive velocity. Andrews and Horsfield [3] utilized a single-frame long exposure camera with a halogen light lamp in order to measure abrasive particle trajectory and velocity. Andrews also developed a particle correlation method that utilized an optical sensor in order to determine the distribution of sand grain velocity in a sand-blast operation [93]. Ghobeity et al. [94] applied a phase-Doppler particle analyzer (PDPA), and Dehnadfar et al. [95] utilized double-pulse laser shadowgraphy in order to measure abrasive velocity distribution. No attempt at measuring particle orientation was made in any of these studies.

In summary, although the behaviour of spherical and non-spherical particles in fluids has been measured and modeled in past investigations, the studies mostly focused on Stokes flow. Very few considered flow regimes approaching those present in solid particle erosion testing and AJM applications, and none conducted measurements of instantaneous particle orientation in such 
flows. While previous studies have identified initial orientations of angular particles that are most likely to give rise to embedding, none considered what process parameters are likely to result in these particular orientations. The aims of this paper were to address this question, by, for the first time, measuring and modeling the distribution of instantaneous angular particle orientations in an abrasive air jet under conditions that are typical of solid particle erosion testing and abrasive jet machining applications.

\subsection{Experiments}

\subsubsection{Particle characterization}

Four sizes of angular silicon carbide $(\mathrm{SiC})$ powders $($ Grit size $=60,90,120,180)$ were used in the experiments. An optical particle sizing system (Clemex PSA Research Unit, Clemex Technologies Inc., Longueuil, Quebec, Canada) was used to obtain the in-plane distribution of area, circular diameter $\left(D_{\text {Circular }}=2 \sqrt{\text { Area } / \pi}\right)$, and roundness $\left(R=\frac{4 \pi \text { Area }}{\text { Perimeter }^{2}}\right)$. Figure 2.1 shows a sample image of the particles obtained using this system.

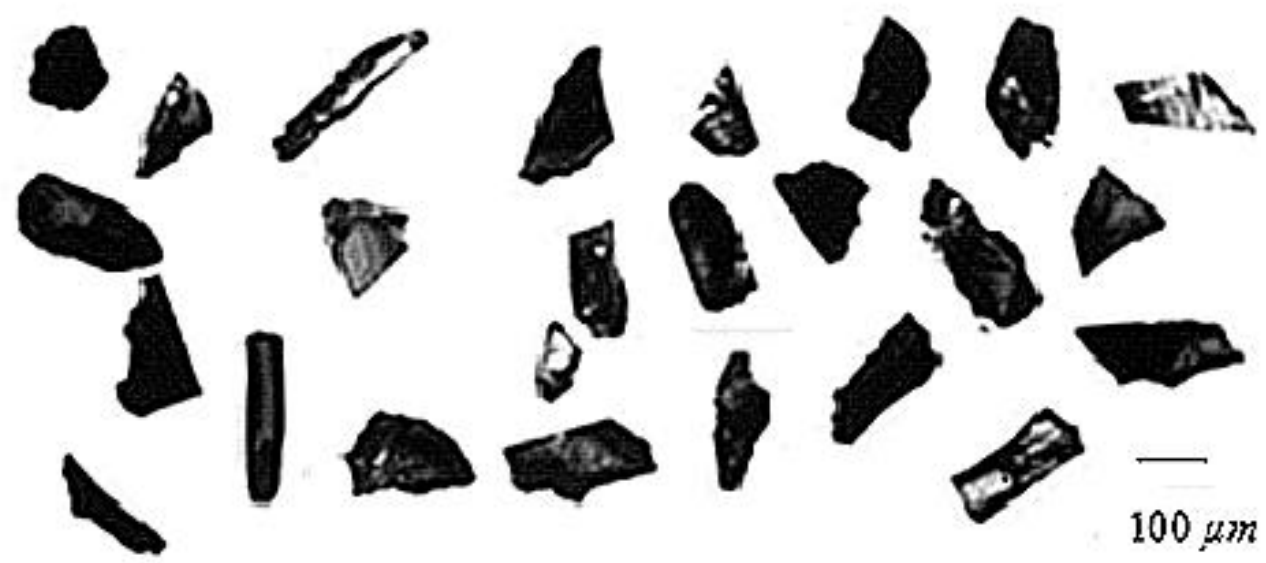

Figure 2.1. Geometry of 90 Grit SiC particles.

The distribution of the average particle out of plane thicknesses was calculated based on noncontact optical profilometer (Nanovea ST400, Micro Photonics Inc., Allentown, PA, USA) measurements of the ratio of the volume to the in-plane area. Parameters describing the obtained distributions of these parameters for all the powders utilized in the experiments are provided in 
Appendix A. As an example, Fig. 2.2 shows the distribution of the $D_{\text {Circular }}$, Area, $R$, thickness and aspect ratio (AR, the ratio of longest to shortest feret) for three particle samples ( 90 grit) containing approximately 1000 particles.

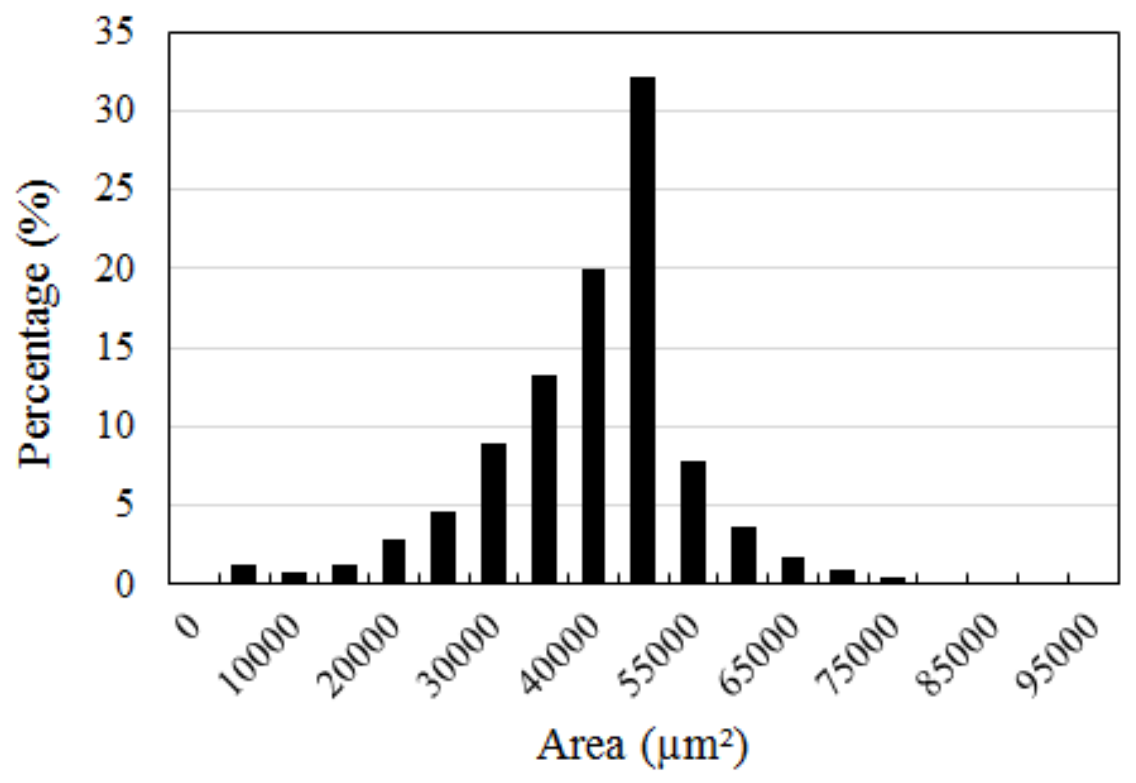

(a)

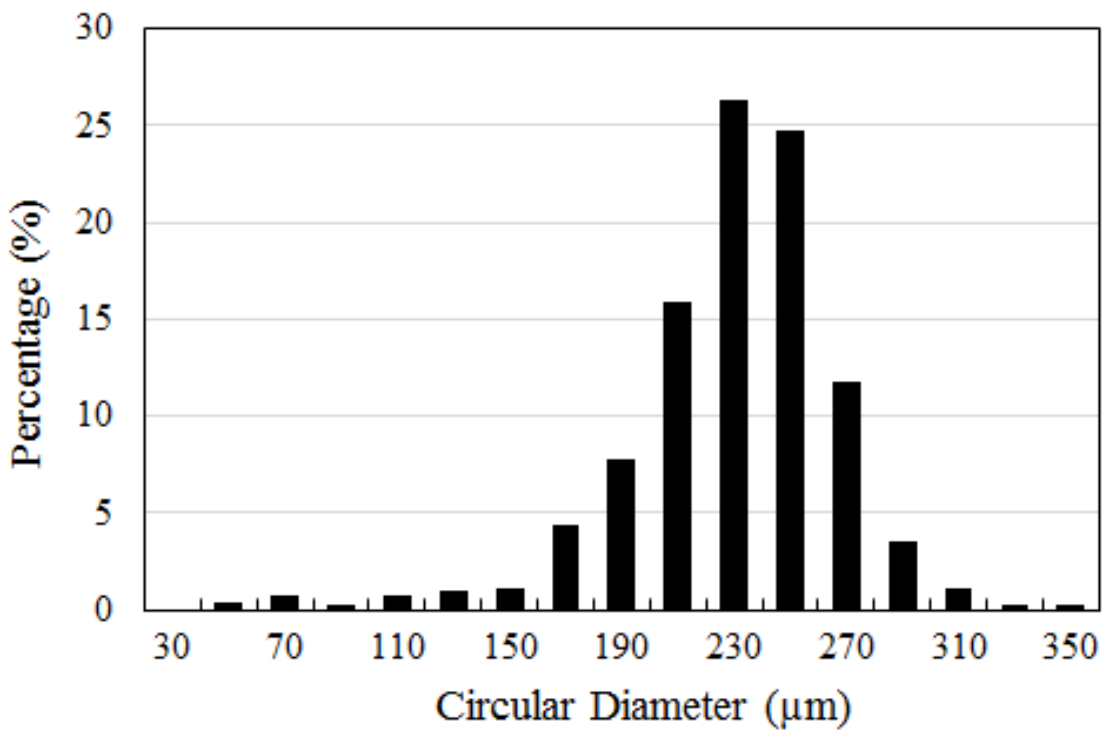

(b) 


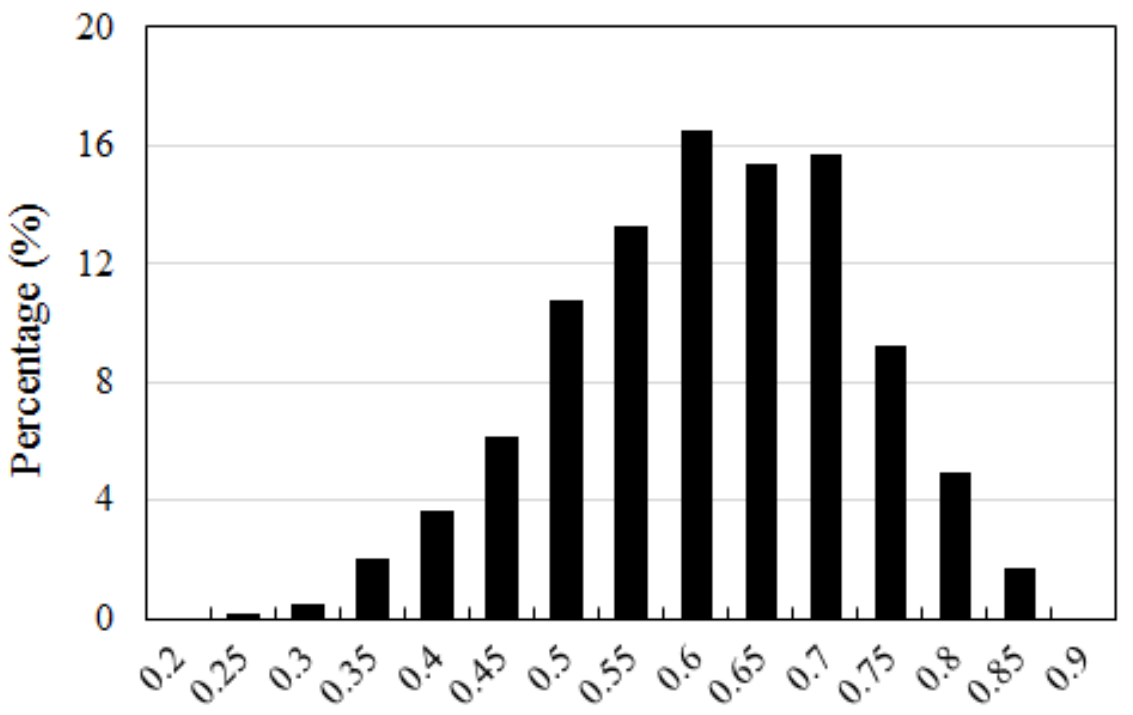

Roundness

(c)

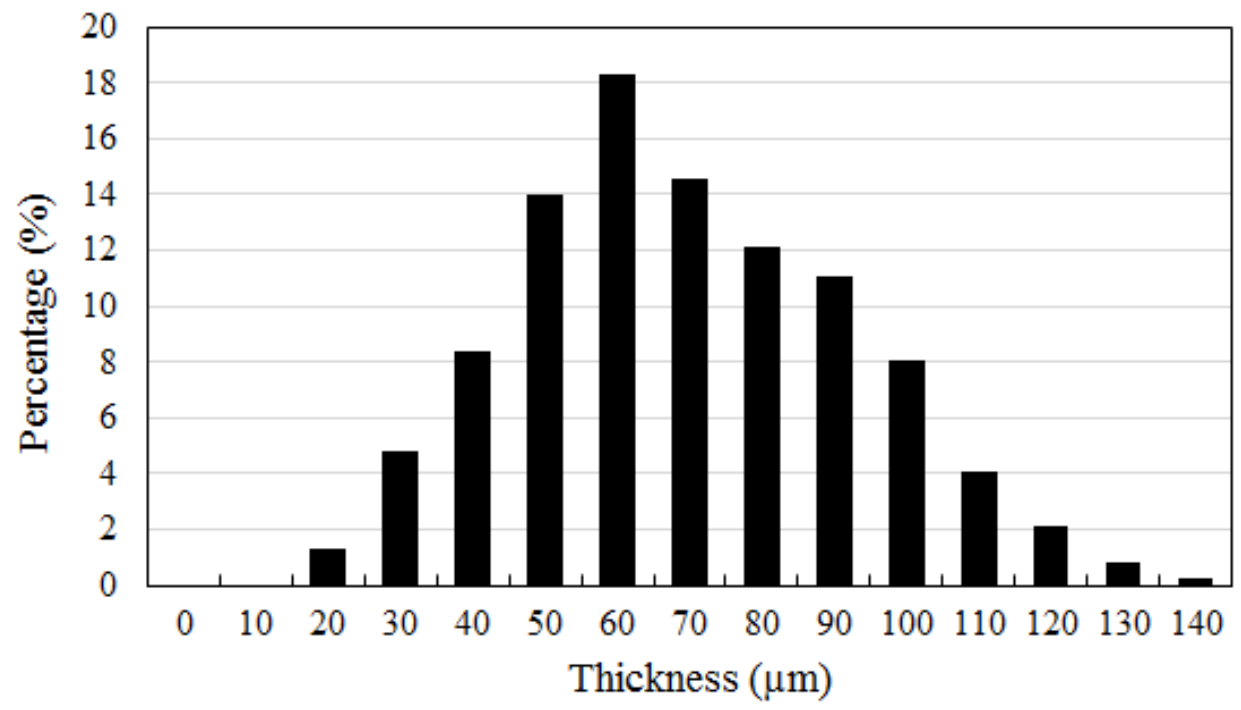

(d) 


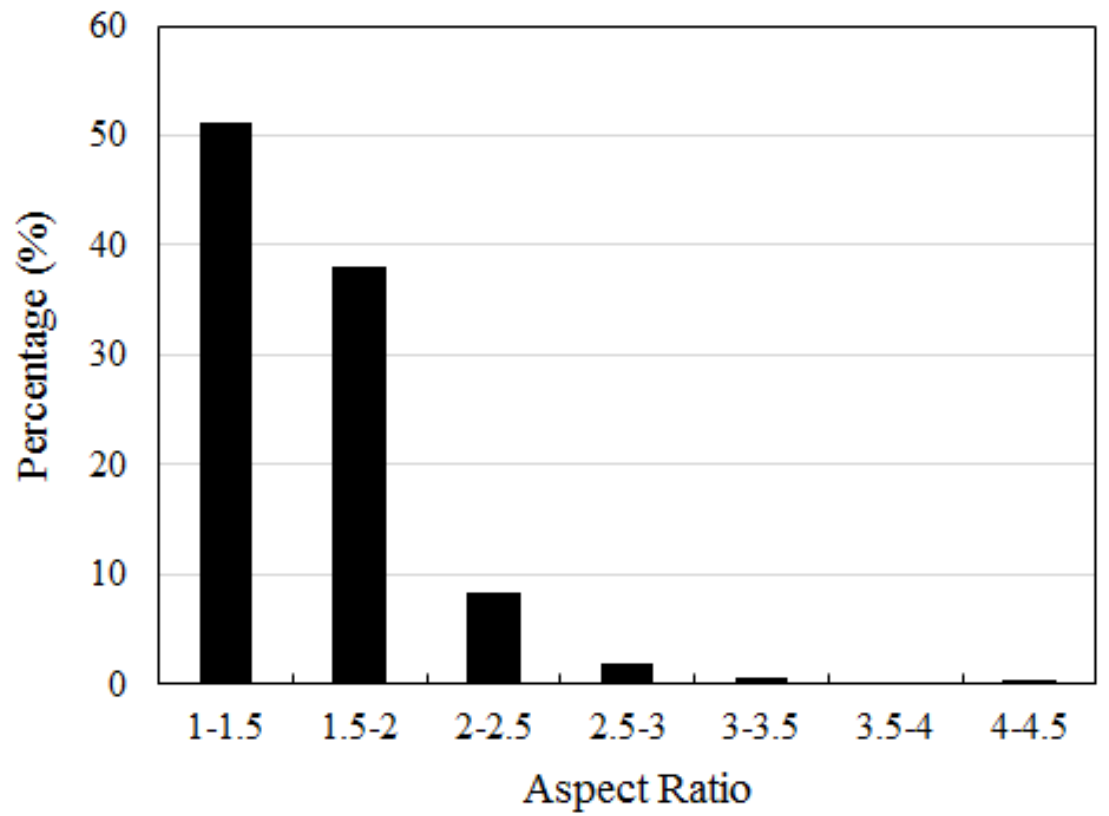

(e)

Figure 2.2. Distribution of: (a) in-plane area; (b) circular diameter; (c) roundness; (d) average thickness; (e) aspect ratio for 90 grit $\mathrm{SiC}$ abrasive.

For all grit sizes, the average thickness was 3-4 times less than the average circular diameter, indicating that the particles were flaky, rather than blocky.

\subsubsection{Shadowgraphy Measurements}

\subsubsection{Apparatus}

The behaviour of the airborne particles within the jet was studied using images obtained from double-pulsed laser shadowgraphy. The jet was formed by a commercial micro-abrasive blaster (Accuflo, Comco Inc., Burbank, CA, USA) operating between 100 and $500 \mathrm{kPa}$, utilizing a $40 \mathrm{~mm}$ long, $1.5 \mathrm{~mm}$ diameter round nozzle. The mass flow rate for each set of conditions was measured by collecting and weighing the particles exiting from the nozzle during a measured time interval, and ranged from $0.41 \mathrm{~g} / \mathrm{min}$ for the smallest particles (grit 180) to $3.12 \mathrm{~g} / \mathrm{min}$ for the largest particles (grit 60). Under these conditions, the average distance between the particles in flight varied between $2 \mathrm{~mm}$ to $10 \mathrm{~mm}$, thus ensuring that there was very little, if any, interaction between them. 
The details of the shadowgraphy apparatus can be found in Ref. [95]. Briefly, a doublepulsed frequency-double Nd: YAG (neodymium:yttrium aluminum garnet) laser, capable of generating a maximum $0.3 \mathrm{~J} /$ pulse pair at $1000 \mathrm{~Hz}$ was coupled to a high efficiency diffuser (Item No.: 1108417, Lavision GmbH, Goettingen, Germany). As shown in Fig. 2.3, the diffuser was placed directly opposite a high speed CCD camera (Imager Pro PlusX, Lavision GmbH, Goettingen, Germany) fitted with a high magnification zoom lens (Navitar zoom 12x, Navitar Inc., Rochester, New York, USA) such that the axis of the diffuser and lens of the CCD camera were aligned. The jet of particles was made to pass in a polycarbonate chamber placed between the diffuser and CCD camera such that the particles on the focal plane of the lens were illuminated by the laser pulses. The laser pulse duration was $1 n s$, and, depending on experimental conditions, the time intervals between pulses were generally in the range of 1-4 $\mu s$. For measurements of rotation angle, the time interval was increased to $15 \mu \mathrm{s}$. In this manner, multiple sets of two images of the particles in flight were obtained and used to determine the particle velocity and orientation.

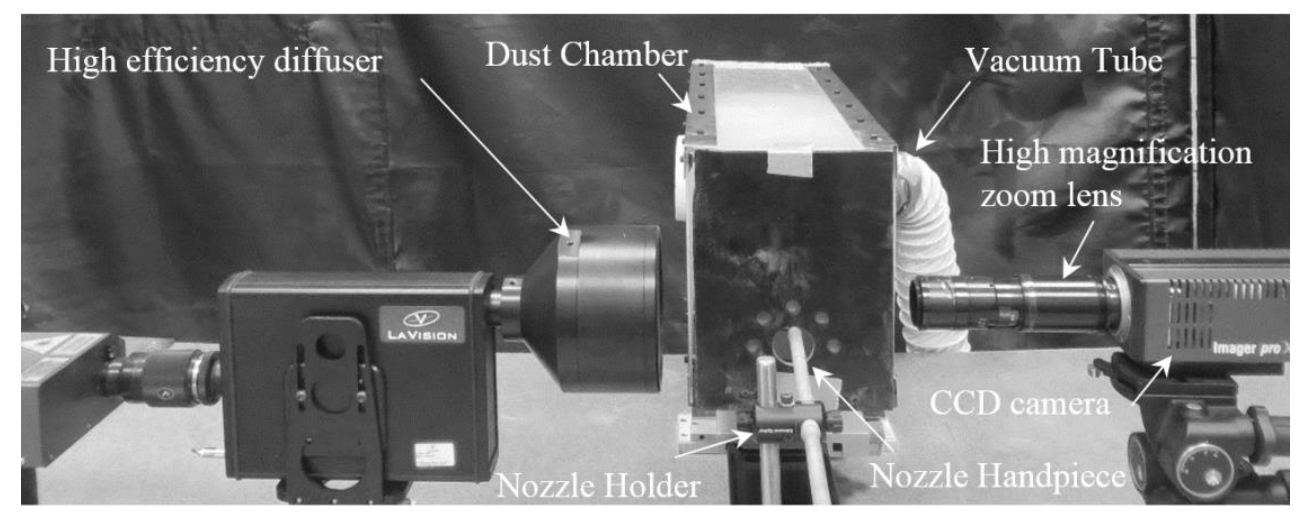

Figure 2.3 Shadowgraphy set up for measurement of particles velocity and orientation.

Shadowgraphic measurements were made on airborne particles within abrasive jets utilizing the four sizes of $\mathrm{SiC}$ particles described in Section 2.1, each using 5 different pressures and at 5 different standoff distances from the nozzle exit (Table 2.1). All measurements at a given combination of particle size, air pressure and standoff were repeated at least twice. Analyses were performed based on 2000 image pairs, since comparison to analyses using 3000 images yielded differences in measured quantities that were at most $6.8 \%$. 
Table 2.1 Combination of process parameters used in the shadowgraphy and embedding measurements. Each condition was used for all four particles sizes described in Appendix A.

\begin{tabular}{|c|c|c|c|c|c|}
\hline \multirow{2}{*}{$\begin{array}{c}\text { Distance } \\
\text { from } \\
\text { Nozzle exit } \\
(\mathrm{mm})\end{array}$} & \multicolumn{5}{|c|}{ Jet Pressure $(k P a)$} \\
\hline & 100 & 200 & 300 & 400 & 500 \\
\hline 0 & $*$ & $*$ & $\checkmark \quad *$ & $*$ & $*$ \\
\hline 10 & $*$ & $*$ & $\checkmark *$ & $*$ & * \\
\hline 20 & $*$ & $*$ & $\checkmark *$ & $*$ & $*$ \\
\hline 30 & $*$ & $*$ & $\checkmark *$ & $*$ & $*$ \\
\hline 40 & $*$ & $*$ & $\checkmark *$ & $*$ & $*$ \\
\hline \multicolumn{6}{|c|}{$\begin{array}{l}* \text { : shadowgraphy experiment } \\
\checkmark \text { : embedding experiment }\end{array}$} \\
\hline
\end{tabular}

\subsubsection{Analysis of the shadowgraphy images}

The particle linear velocity distribution was measured from the image pairs using Davis Software (Lavision GmbH, Goettingen, Germany) and, in order to evaluate the instantaneous angular position of the particles within the jet, the images were also imported into Clemex PSA Professional Research Particle Size and Shape Image Analysis software (Clemex Technologies Inc., Longueuil, Quebec). Two different measures of the angular position were used, the alignment angle and the orientation angle. The alignment angle was defined as the angle between the line along the longest orthogonal distance between any two points on the edge of the particle (longest feret in Fig. 2.4a) and the velocity vector, which was approximately parallel to the jet axis. The alignment angle reflects the likelihood of aerodynamic alignment since it is defined based on the direction of the longest particle dimension relative to the flow direction. Particle embedment, on the other hand, depends more on the location of the particle center of mass relative to its leading vertex (the assumed target impact point), rather than the location of the longest feret. To reflect this, an 'orientation angle' (Fig. 2.4b) was measured as the angle between the jet axis, i.e. the particle velocity vector, and the line connecting the furthest downstream particle vertex and the particle centroid. In this scheme, a zero orientation angle indicates a perfectly 'oriented' particle with its velocity vector parallel to the line connecting the leading vertex and the center of mass. This definition is consistent with that used by Getu et al. [16] in their analytical studies of embedding. 
The $\mathrm{X}$ and $\mathrm{Y}$ coordinates of points defining the perimeters (Section 2.3), centers of mass, longest ferets, and leading vertices of the particles were obtained from analysis of the shadowgraphy images using the Clemex software. A Matlab R2013a (Mathworks) routine was developed to determine the resulting distribution of orientation and alignment angles. Measurement of orientation and alignment angle distributions were based on their absolute values, i.e. direction of rotation was not considered, and the optimum bin size was chosen based on the Freedman-Diaconis rule [96].

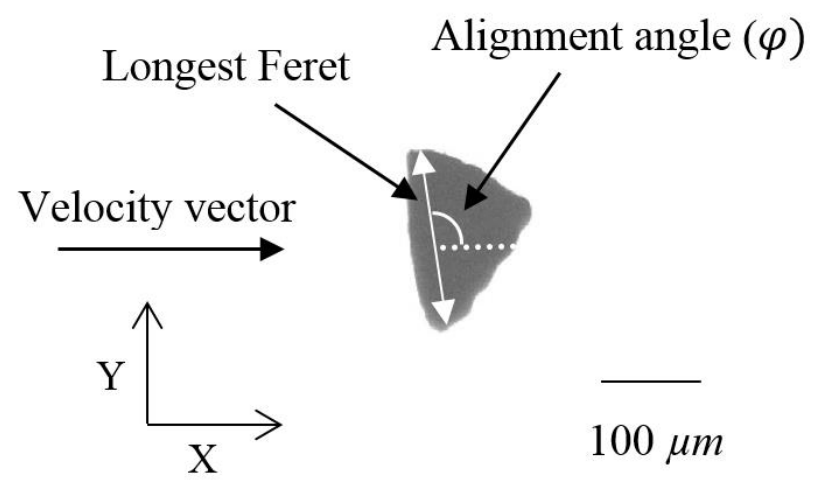

(a)

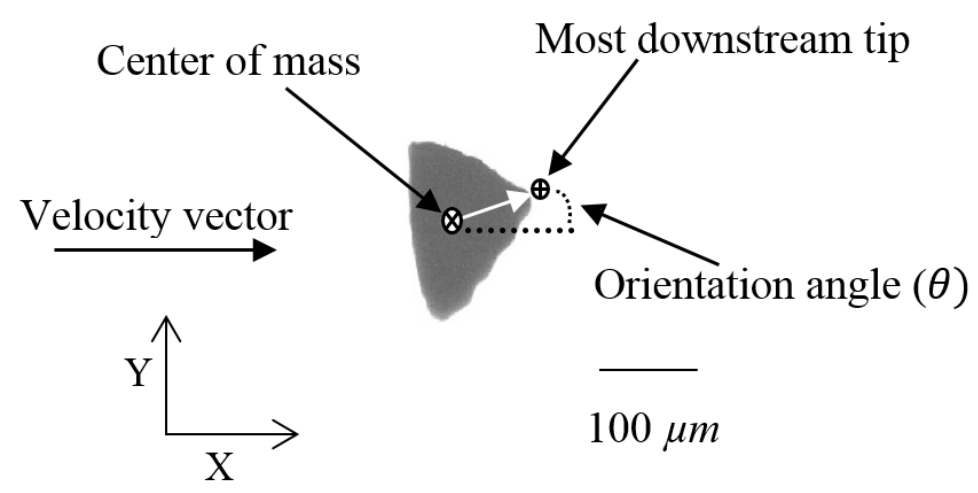

(b)

Figure 2.4 Definition of (a) alignment angle $\varphi$, and (b) orientation angle $\theta$ for a typical particle.

The angular position distributions represent an instantaneous snapshot at a given standoff and cannot be used to infer whether the particles were rotating. Therefore, for grit 60 particles at 
the nozzle exit, 20 and $40 \mathrm{~mm}$ standoff, the particle angular displacements (rotation angle) about their centers of mass that occurred during the interval between laser pulses were also measured by considering the double pulse image pairs. To ensure that particle rotations were only measured in the plane parallel to the velocity vector, only particles with less than $20 \%$ variation in planar area measured in successive images were used. To ensure that the rotation of particle could be detected, the time interval between the two laser pulses was set at $15 \mu s$ so that the distance traveled by the particle was 3-4 times larger than the particle dimensions.

\subsubsection{Particle embedment experiments}

In order determine whether orientation could be correlated with particle embedment, 5.5 $m m$ thick Al 6061-T6 (90 BHN) samples were subjected to short bursts of the SiC abrasive powders using the same setup described in Section 2.2.2.1 at a pressure of $300 \mathrm{kPa}$ (Table 2.1). The samples were polished to a roughness of $0.02 \mu \mathrm{m}$ and a programmable shutter device was utilized so that the substrate would be exposed to the burst of abrasives for less than $12.5 \mathrm{~ms}$ to ensure that individual embedded particles and impact craters could be identified. All blasting was performed with the jet incident perpendicular to the target. After being exposed to the burst, the samples were cleaned with distilled water and dried using compressed air to ensure that all the dust and/or deposited abrasives were removed.

Scanning electron micrographs (SEM's) were taken of the blasted surfaces, and the embedded particles were identified and counted using energy dispersive $\mathrm{x}$-ray spectroscopy (EDX) to confirm the presence of Si. The number of launched particles was determined in a similar manner, except that the number of impact sites (sum of the identified impact craters and embedded particles) were counted. The percentage of embedded particles was determined as the ratio of the number of embedded particles to the total number of launched particles. A calculation of the number of blasted particles based on the average particle size, the measured particle mass flow rate, the particle density, and the exposure time, typically yielded results that were within $8 \%$ of the manually counted impact sites. Figure 2.5 indicates that most of the embedded particles penetrated into the target in the direction perpendicular to their thickness. 


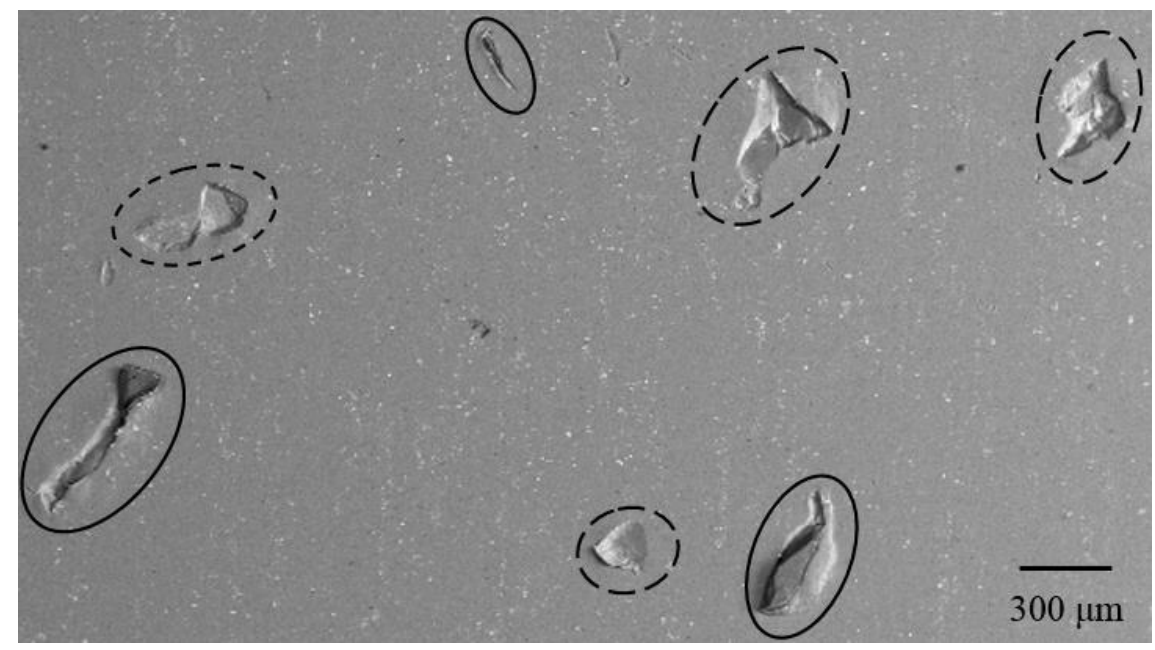

Figure 2.5 SEM - showing embedded SiC particles (solid lines) and impact craters without embedded particles (dashed lines) in Al6061-T6.

\subsection{Model to predict particle orientation}

Li et al. [97] developed a model that predicts the linear velocity of spherical particles in an abrasive flow, based on steady and one-dimensional compressible air-particle flow in a frictionless nozzle, ignoring the collisions between abrasive particles or with the nozzle. The model utilizes the particle and nozzle dimensions, the input pressure and the air flow rate in order to determine the air and particle velocities inside the nozzle and in the free jet. The distance from where the particles are fed into the nozzle to the desired nozzle standoff is divided into cells, and in each cell, the air velocity and resulting particle drag force are used to calculate the acceleration, and thus the linear velocity of the particles. The equations of motion are solved in each cell, until the air and particle velocity are obtained at any position both within and after exiting the nozzle.

The approach of Li et al. [97], developed for nonrotating spherical particles, was modified in the present work to take into account angular particle shape, and rotational motion. The main complication in introducing rotating angular particles was in the calculation of the drag coefficient, which not only depended on the individual particle shape, but also changed instantaneously with orientation as the particle rotated and the frontal area changed. These variations in instantaneous drag coefficient not only influenced the rotational particle motion, but also the translational. For simplicity, it was assumed that all particles travelled with their center of mass on the jet centerline. The translation and rotation of any given particle about its center of mass in any given cell caused 
by the resultant drag force was calculated based on the relative position of the center of mass and the center of pressure of the particles (Fig. 2.6). The model was executed using the following steps:

(i) The skeleton point representation of the particle geometries, i.e. the $X$ and $Y$ coordinates of the perimeter of the particles, were determined from the analysis of the shadowgraphic images of particles at the nozzle exit (Section 2.2.2). For each particle, between 150 and 250 points were used to define the particle perimeter. Therefore, the shapes of the particles used in the simulation were two-dimensional projections of actual particles.

(ii) The interior of the nozzle and the region between the nozzle exit and the desired standoff distance were divided into 2000 cells, so chosen based on convergence studies that ensured a less than $1 \%$ difference in calculated average velocity when 2500 cells were used. In the cell that was furthest upstream within the nozzle, a random alignment angle (Fig. 2.4a) was assigned to each particle which was assumed initially stationary.

(iii) The forces acting on the particle in first cell inside nozzle were found. The equations of motion were solved and the linear velocity, torque and resulting angular acceleration determined, along with the rotation angle and position of center of mass. The torque $(\Psi)$ in any given cell was calculated as:

$\Psi=F_{n} X_{c p}$

where $F_{n}$ is the net drag force and $X_{c p}$ the distance from the center of mass to the center of pressure of the particle, assumed to be located at the mid-point of the instantaneous length of the particle in the $Y$ direction (Fig.2.6). $\quad F_{n}$ was assumed to act along the jet axis, and be constant in a given cell as [97]:

$F_{n}=0.5 \rho_{\text {air }}\left(V_{\text {air }}-V_{\text {particle }}\right)^{2} A_{\text {particle }} C_{D}$ 
where $\rho_{\text {air }}$ is the air density. The air and particle velocities, $V_{\text {air }}$ and $V_{\text {particle }}$, were calculated following the procedure Li et al. [97], except that the instantaneous alignment-dependent equations for drag coefficient, $C_{D}$, presented in Appendix B were used rather than a constant drag coefficient. The instantaneous projected frontal area of the particle, $A_{\text {particle }}$, was determined as the product of the measured average particle thickness and the instantaneous height, calculated from the skeleton point representation of the particle which, as discussed below, was updated in each cell, according to the instantaneous alignment angle $(\varphi)$, between the particle major axis (i.e. longest feret of particle), $L$, and the jet axis. The particle angular acceleration $\ddot{\alpha}$, velocity $\dot{\alpha}$ and rotation angle $\alpha$ about the center of mass were calculated using:

$\Psi=I \ddot{\alpha}$

$\dot{\alpha}(t+d t)=\left(\frac{\ddot{\alpha}(t+d t)+\ddot{\alpha}(t)}{2}\right) d t+\dot{\alpha}(t)$

$\alpha=\dot{\alpha} d t$

where $I$ is the moment of inertia of the particle and $d t$ is the time it takes for the particle to travel the very small length of one cell.

In each cell, the skeleton point representation of the particle periphery was updated considering the rotation occurring in the previous cell by multiplying by an appropriate rotation matrix. The updated instantaneous alignment angle and projected area were utilized at the beginning of next cell to calculate (step iii) the drag force, linear velocity and corresponding rotation angle for that cell. This nested loop was repeated for all the cells to a standoff distance of $40 \mathrm{~mm}$ to obtain the orientation and alignment angles, and the linear and rotational velocities and accelerations in each cell for 200 particles, each of grit size 60 and 90. 


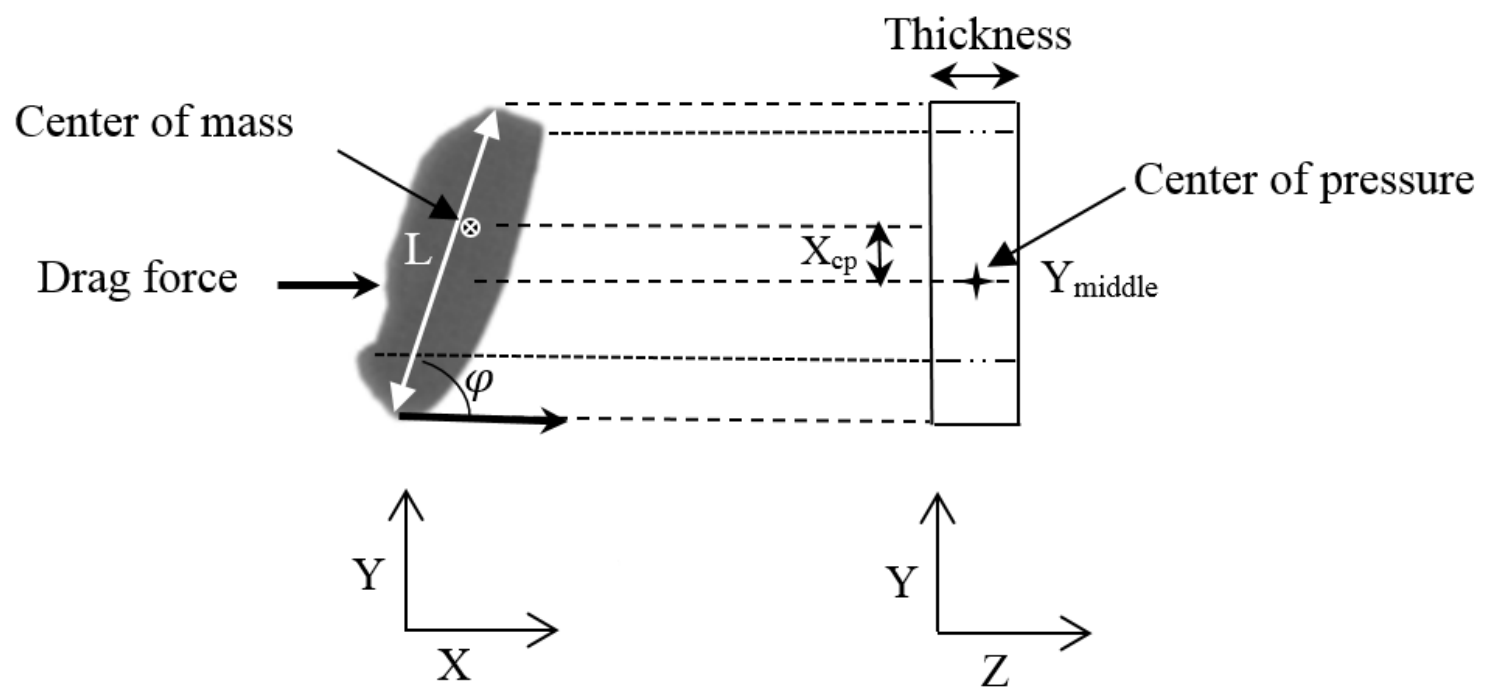

Figure 2.6 Schematic defining particle parameters used in model.

The orientation angle of a given particle as defined in Fig. 2.4b was predicted by determining, at the cell corresponding to the desired standoff, the positions of the most downstream vertex and center of mass of the particle. The predicted orientation angles at different standoffs could then be compared with the corresponding shadowgraphic measurements.

\subsection{Results and discussion}

\subsubsection{Particle linear velocity}

The measured average linear particle velocities at different standoffs and pressures are given in Table 2.2. For the 0 to $40 \mathrm{~mm}$ range of standoff distances, the particle velocity increased with the standoff distance in most cases, implying that the net drag force acted furthest upstream side of the particle, tending to accelerate and rotate the particles. Figure 2.7 shows the trend for two particle sizes at $300 \mathrm{kPa}$, together with the results from the model of Section 2.3. For all particle sizes, the model was able to predict the linear velocities to within a maximum error of $15 \%$. The error is likely due to the fact that the same calibration factor for drag coefficient (Appendix B) was used for both grit 60 and grit 90 particles, resulting in over-estimates for the larger particle size and underestimates for the smaller. 
Table 2.2 Average particle linear velocity $(\mathrm{m} / \mathrm{s})$ of particles at different standoffs and jet pressures.

\begin{tabular}{|c|c|c|c|c|c|c|}
\hline \multirow{3}{*}{$\begin{array}{l}\text { Grit } \\
\text { size }\end{array}$} & \multirow{3}{*}{$\begin{array}{c}\text { Pressure } \\
\qquad(k P a)\end{array}$} & \multicolumn{5}{|c|}{ Average Particle Velocity $(\mathrm{m} / \mathrm{s})$} \\
\hline & & \multicolumn{5}{|c|}{ Standoff $(\mathrm{mm})$} \\
\hline & & $\begin{array}{c}0 \\
\text { (Nozzle } \\
\text { exit) }\end{array}$ & 10 & 20 & 30 & 40 \\
\hline \multirow{5}{*}{60} & 100 & 34 & 36 & 38 & 41 & 39 \\
\hline & 200 & 60 & 66 & 67 & 69 & 67 \\
\hline & 300 & 76 & 83 & 87 & 89 & 89 \\
\hline & 400 & 87 & 96 & 100 & 106 & 106 \\
\hline & 500 & 67 & 108 & 112 & 116 & 115 \\
\hline \multirow{5}{*}{90} & 100 & 64 & 69 & 71 & 81 & 89 \\
\hline & 200 & 88 & 90 & 96 & 101 & 106 \\
\hline & 300 & 101 & 110 & 120 & 128 & 136 \\
\hline & 400 & 110 & 116 & 125 & 131 & 141 \\
\hline & 500 & 122 & 136 & 147 & 152 & 159 \\
\hline \multirow{5}{*}{120} & 100 & 74 & 78 & 80 & 86 & 94 \\
\hline & 200 & 93 & 101 & 104 & 105 & 112 \\
\hline & 300 & 108 & 114 & 131 & 140 & 150 \\
\hline & 400 & 130 & 134 & 146 & 156 & 169 \\
\hline & 500 & 148 & 168 & 175 & 180 & 187 \\
\hline \multirow{5}{*}{180} & 100 & 93 & 101 & 104 & 106 & 109 \\
\hline & 200 & 120 & 128 & 131 & 136 & 142 \\
\hline & 300 & 136 & 141 & 155 & 165 & 177 \\
\hline & 400 & 159 & 166 & 180 & 187 & 196 \\
\hline & 500 & 167 & 185 & 194 & 201 & 208 \\
\hline
\end{tabular}

As expected, increases in pressure led to increases in particle linear velocity; e.g., the average velocity of grit 90 particles at $300 \mathrm{kPa}$ increased from $110 \mathrm{~m} / \mathrm{s}$ to $136 \mathrm{~m} / \mathrm{s}$ as the standoff changed from $10 \mathrm{~mm}$ to $40 \mathrm{~mm}$. At the same pressure and standoff, as expected, the particle 
velocity increased with decreasing particle size. These trends generally agree with the model of Li et al. [97] for spherical particles, and a modification of this model introduced by Dehnadfar et al. [95] for non-rotating angular particles.

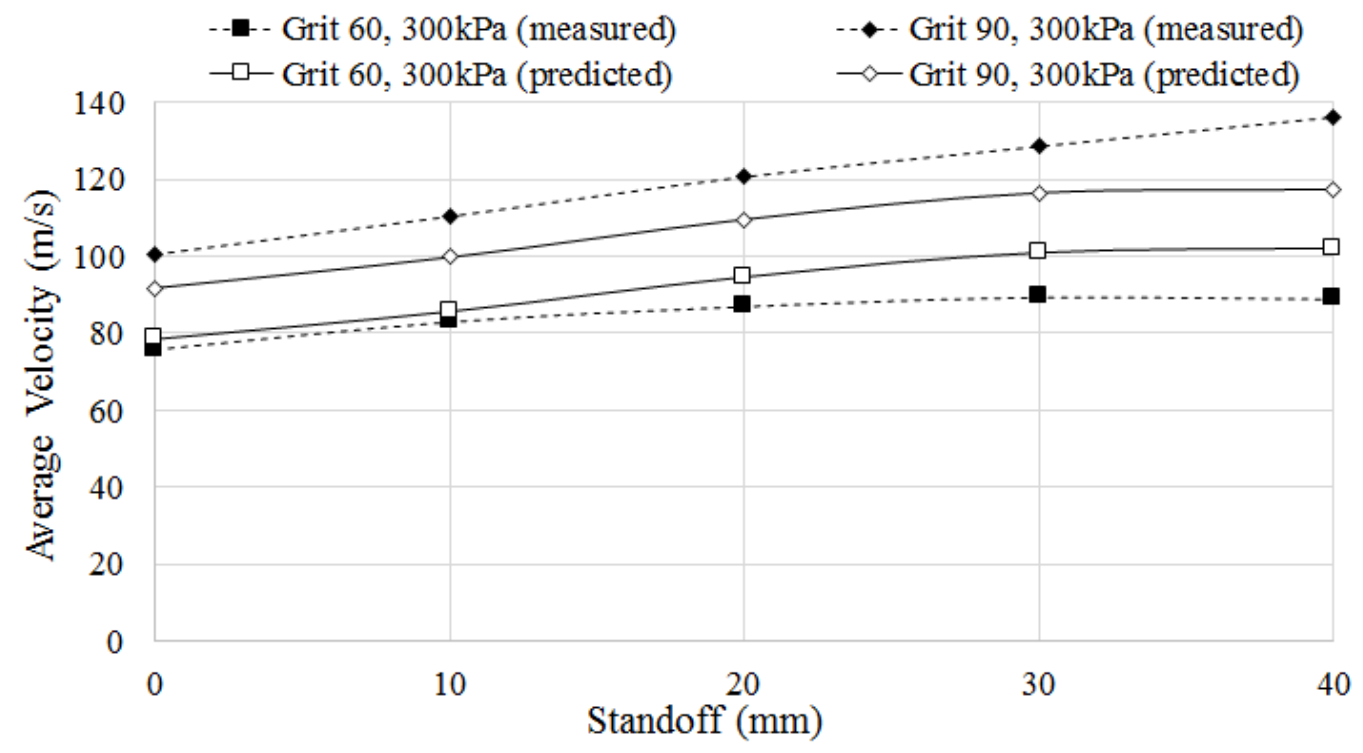

Figure 2.7 Predicted and measured average particle linear velocities for grit 60 and 90 particles at $300 \mathrm{kPa}$.

\subsubsection{Particle rotation angle}

As a representative case, the distribution of measured particle rotation angles at three standoff distances are shown in Figure 2.8 for grit 60 particles. 


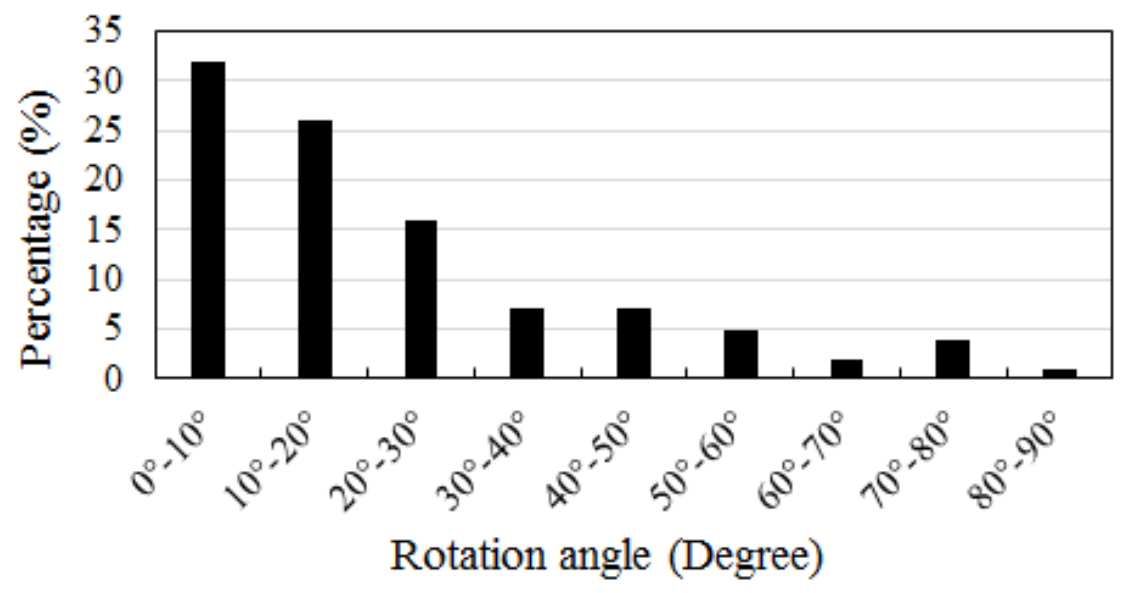

(a)

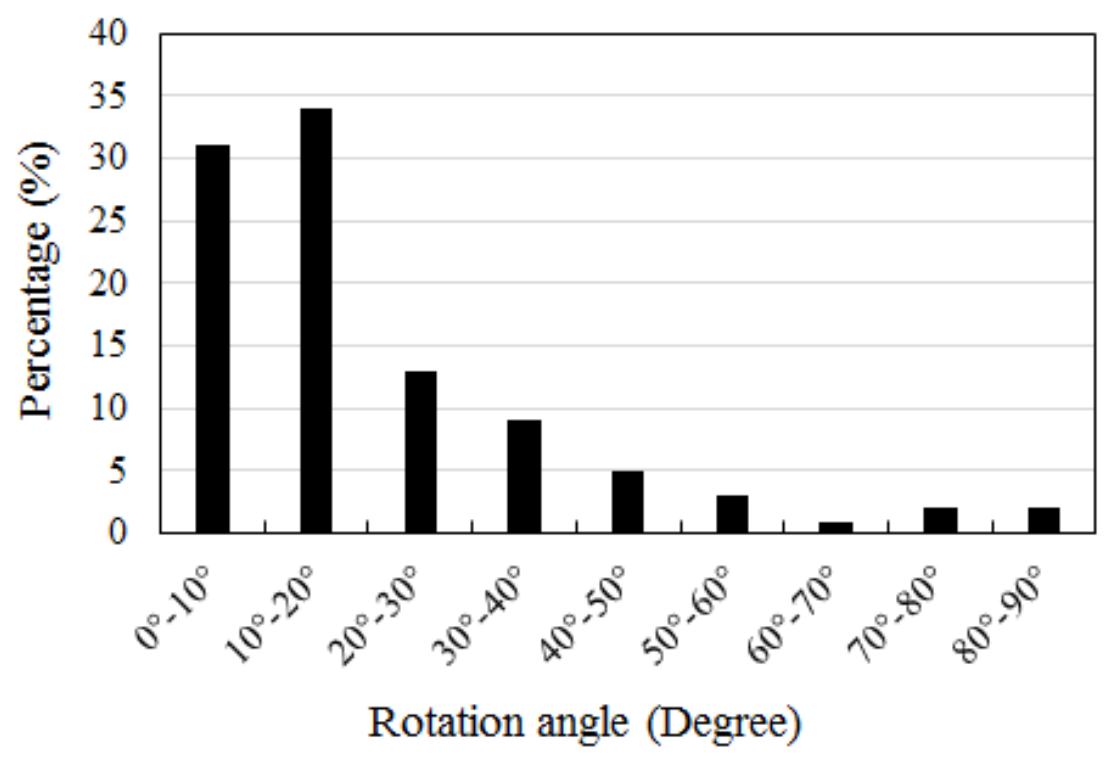

(b) 


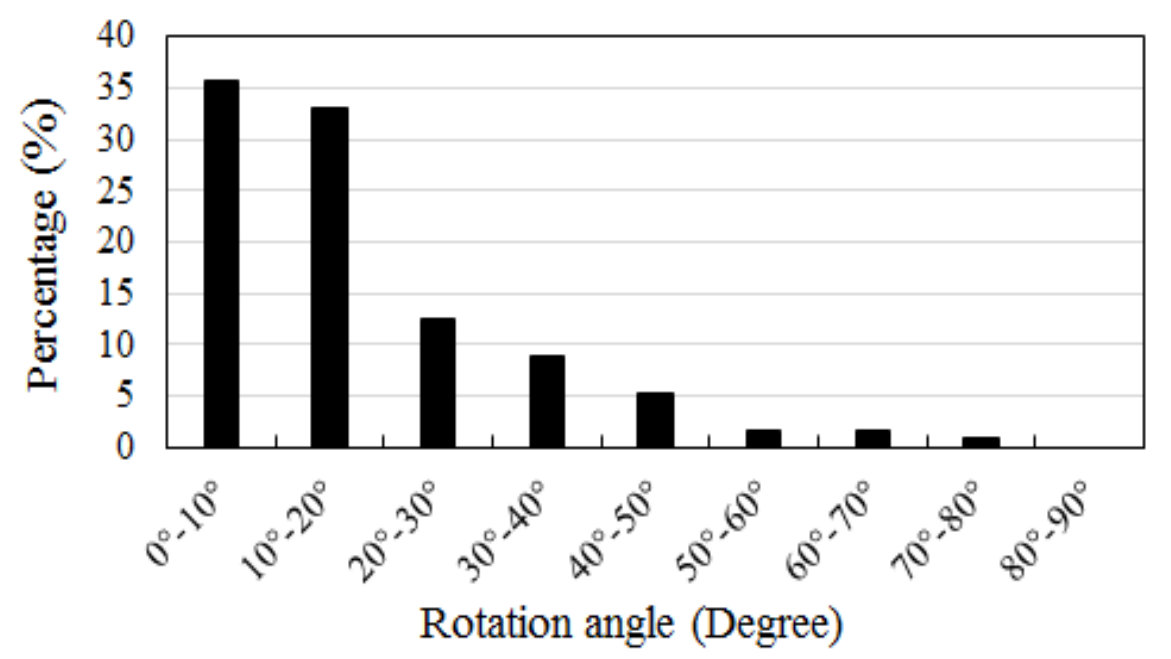

(c)

Figure 2.8 Distribution of angular displacements of grit 60 particles at $300 \mathrm{kPa}$ at the following standoffs (a) nozzle exit; (b) $20 \mathrm{~mm}$; (c) $40 \mathrm{~mm}$. The average linear distance travelled by the particles whilst rotating through these angles was between 1.1 and $1.3 \mathrm{~mm}$.

The distributions follow typical log-normal patterns, with the vast majority of particles rotating less than $20^{\circ}$ over the 1.1-1.3 $\mathrm{mm}$ range of distances over which the measurements were made. There was an approximately $11 \%$ increase (from 58 to 69\%) in particles rotating less than $20^{\circ}$ as the standoff increased from the nozzle exit to $40 \mathrm{~mm}$. This implies a decreased rotational kinetic energy as the particles travel in the jet and became more aligned with the flow. Assuming that particles travel an average distance of $\sim 1.2 \mathrm{~mm}$ during the measurements, then, despite the fact that the average rotational velocity is very high $(\sim 25000 \mathrm{rad} / \mathrm{s})$, it can be concluded that most $(64 \%)$ of the particles rotate less than one full revolution as they travel $20 \mathrm{~mm}$.

\subsubsection{Distribution of alignment angle}

Figure 2.9 shows that, consistent with Mortensen et al. [45], particles that have larger aspect ratios are more likely to align with the direction of fluid flow. For example, there was approximately double the number of particles in the left-most (most aligned) bin for particles having $\mathrm{AR}>2.5$ than those having $\mathrm{AR}<1.5$. This increase is statistically significant ( $\mathrm{t}$-test, $\mathrm{P}$ value $<0.05)$. There was no statistically significant effect of aspect ratio on alignment when the aspect ratio was less than 1.5 . 


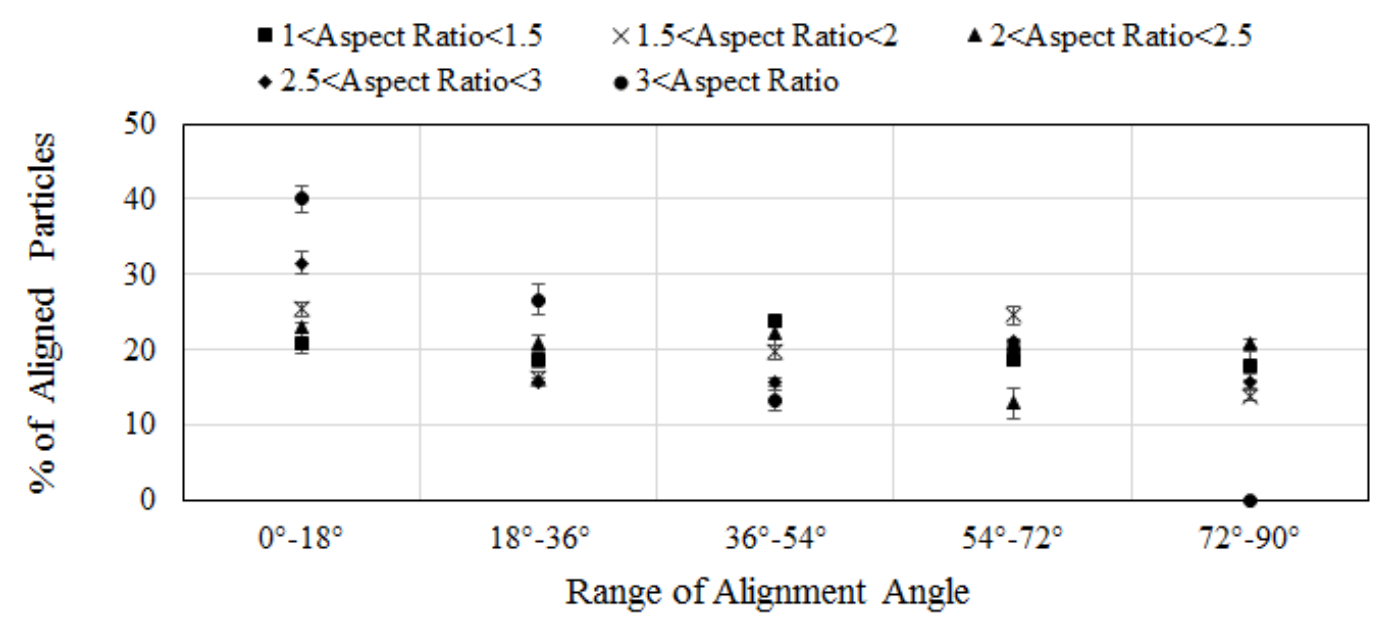

(a)

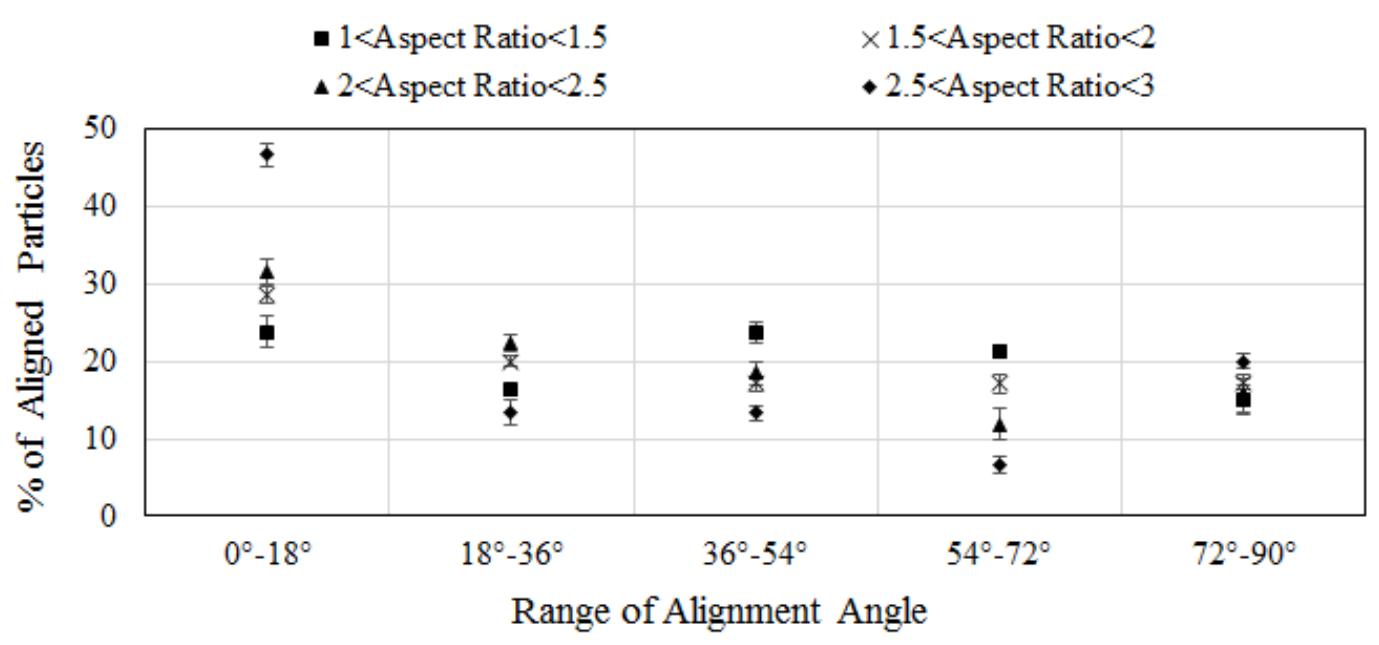

(b)

Figure 2.9 Variation of the alignment angle with aspect ratio at $P=300 \mathrm{kPa}$ and $20 \mathrm{~mm}$ standoff for (a): grit 90; (b): grit 60 particles. Scatter bars show the standard deviation.

\subsubsection{Influence of process parameters on the distribution of orientation angle}

As mentioned in Section 2.2.2, the orientation angle at the moment of impact has been previously shown to strongly affect the material removal mechanism and likelihood of particle embedding in the solid particle erosion of ductile materials [16,28,29,30]. As a representative example, Fig. 2.10 shows the distribution of orientation angle at a $20 \mathrm{~mm}$ standoff for grit 90 particles at 5 different pressures. Similar distributions were obtained for all other particles sizes 
and standoffs. There was a clear tendency for particles to orient themselves with the jet axis at all pressures, i.e. the peaks occurred at low orientation angles. In all cases (i.e., all particles sizes, pressures, and standoffs), the differences in percentages of particles in the first three adjacent bins were all statistically significant ( $\mathrm{t}$-test, $\mathrm{P}$ value $<0.05)$. In most cases, the differences in the relatively small percentage of particles travelling with orientations between $30^{\circ}$ and $90^{\circ}$ were also statistically significant. As can be seen in the example given in Fig. 2.10, in all cases there was also no significant effect (t-test, $\mathrm{P}$ value $<0.05$ ) of pressure on the percentage of the particles at any given range of orientations.

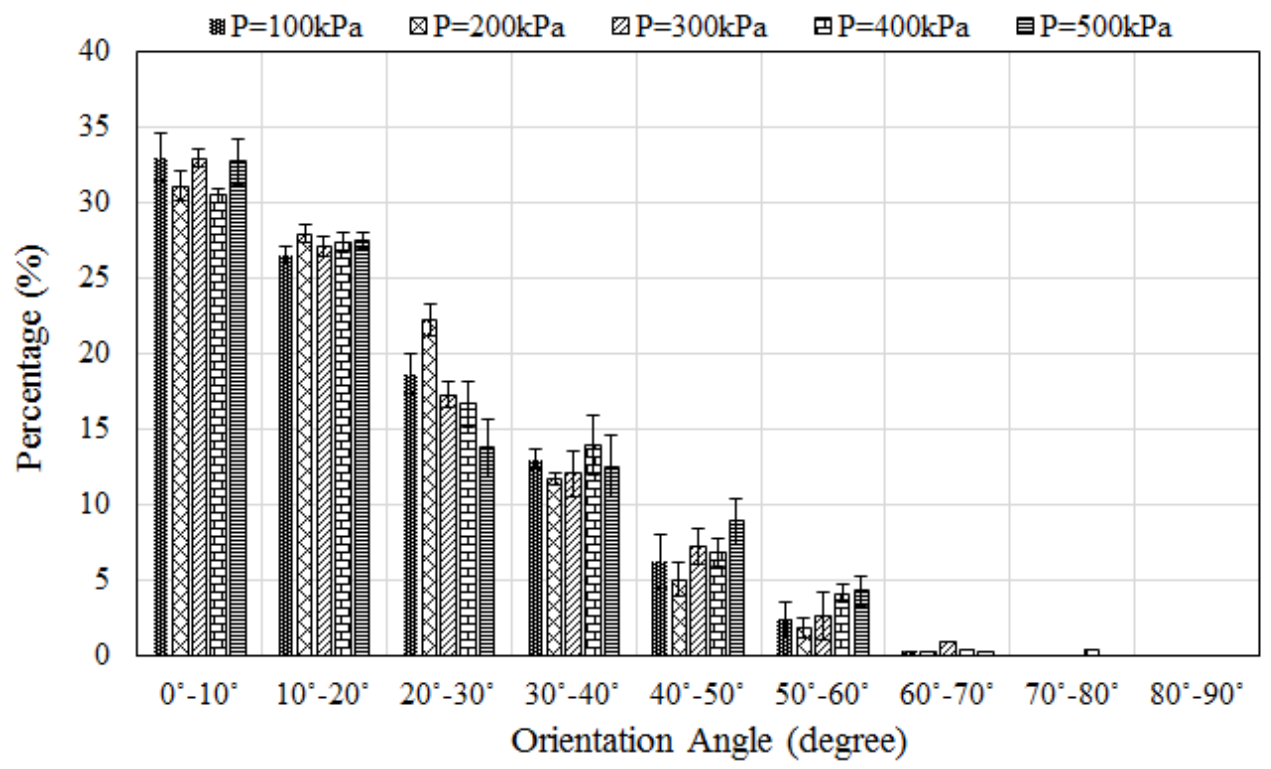

Figure 2.10 Distribution of orientation angle at the standoff of $20 \mathrm{~mm}$ for grit $90 \mathrm{SiC}$ abrasives, Scatter bars show the standard deviation.

Figure 2.11 gives the average percentage of the oriented particles (orientation angle between $0^{\circ}-10^{\circ}$ ) at all air pressures for each particle size and standoff. It shows that larger particles are generally more likely to be oriented with the jet direction at a given standoff than small ones. At all standoffs, the percentage of oriented particles was significantly lower for grit 180 compared to grit 90 and 60, and also grit 120 compared to grit 90 and 60 (t-test, $\mathrm{P}$ value $<0.05$ ). 


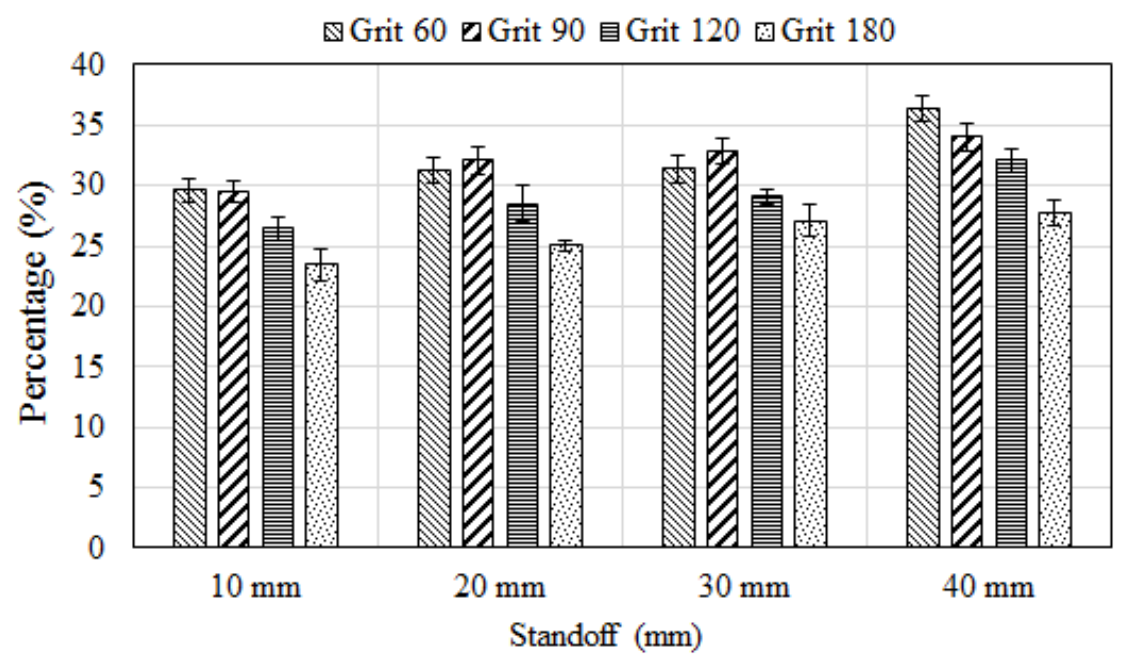

Figure 2.11 Average percentage of oriented particles for different particle sizes at different standoffs. Each bar shows the average of all tested pressures, and scatter bars show the standard deviation.

Figure 2.11 also demonstrates that, for all particle sizes, particles tend to become more oriented with the jet as the standoff is increased. The increase in percentage oriented was statistically significant for any two standoffs with at least $20 \mathrm{~mm}$ difference (t-test, $\mathrm{P}$ value $<0.05$ ). The increase in the number of oriented particles with standoff is expected and consistent with the discussion of the measurements of rotation presented in Section 2.4.2, i.e., the orienting torques due to the drag forces have a longer time to act on the particles.

\subsubsection{Comparison between predicted and measured particle orientation}

Figure 2.12 shows the predicted (model of Section 2.3) and measured orientation distributions of grit 90 and 60 particles at the nozzle exit and at a $40 \mathrm{~mm}$ standoff. The predicted and measured trends are highly consistent, although there was a tendency to slightly over predict the percentage of oriented particles. 


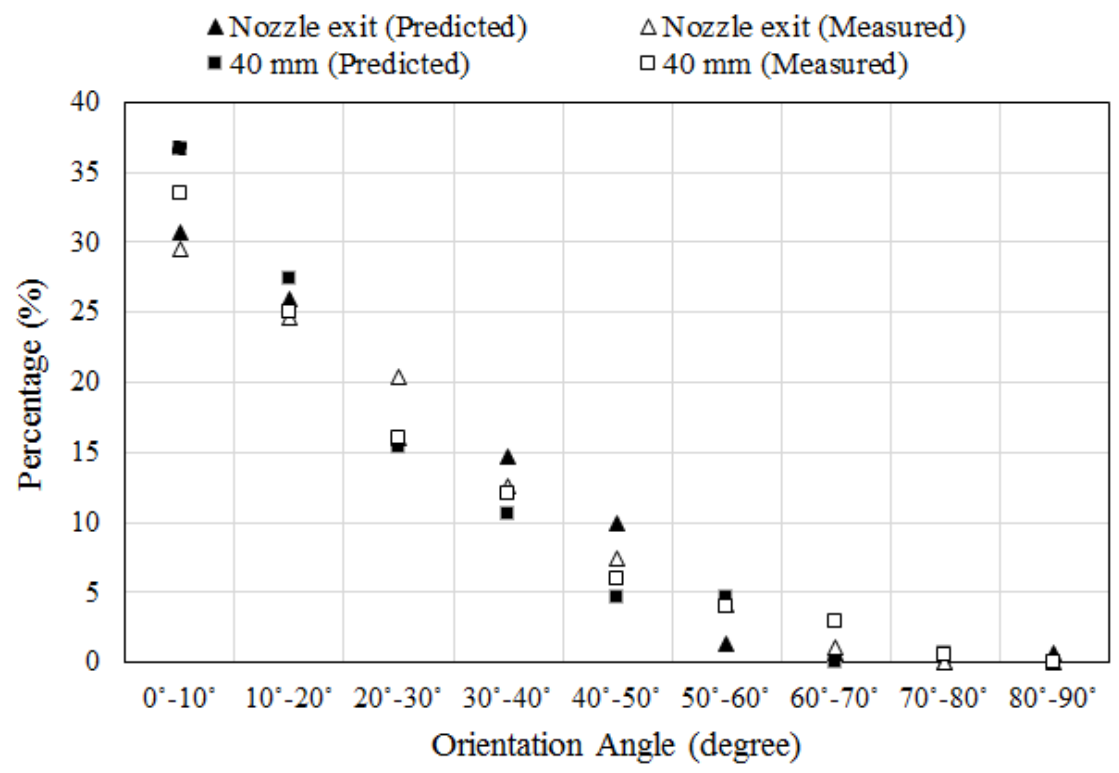

(a)

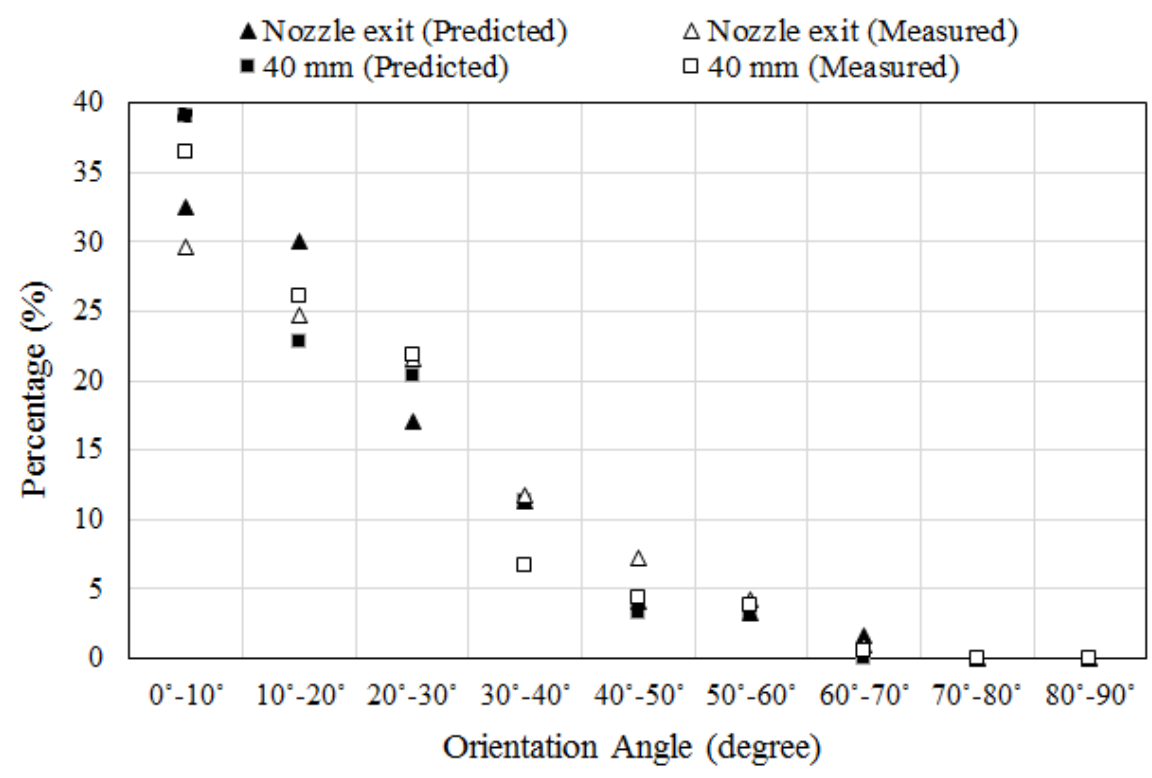

(b)

Figure 2.12 Predicted and measured distribution of particle orientation angles at nozzle exit and $40 \mathrm{~mm}$ standoff for (a) grit 90, and (b) grit 60 particles at $300 \mathrm{kPa}$.

Furthermore, Fig. 2.13 also shows that the model of Section 2.3 was able to quite accurately predict the percentage of oriented particles at any standoff distance. For example, Fig. 2.13 shows 
that, in the worst case, the model predicted approximately $39 \%$ of grit 90 particles oriented with the jet axis at a $40 \mathrm{~mm}$ standoff, while the corresponding measured value was a $36 \%$, i.e. the predicted was 1.08 times the measured.

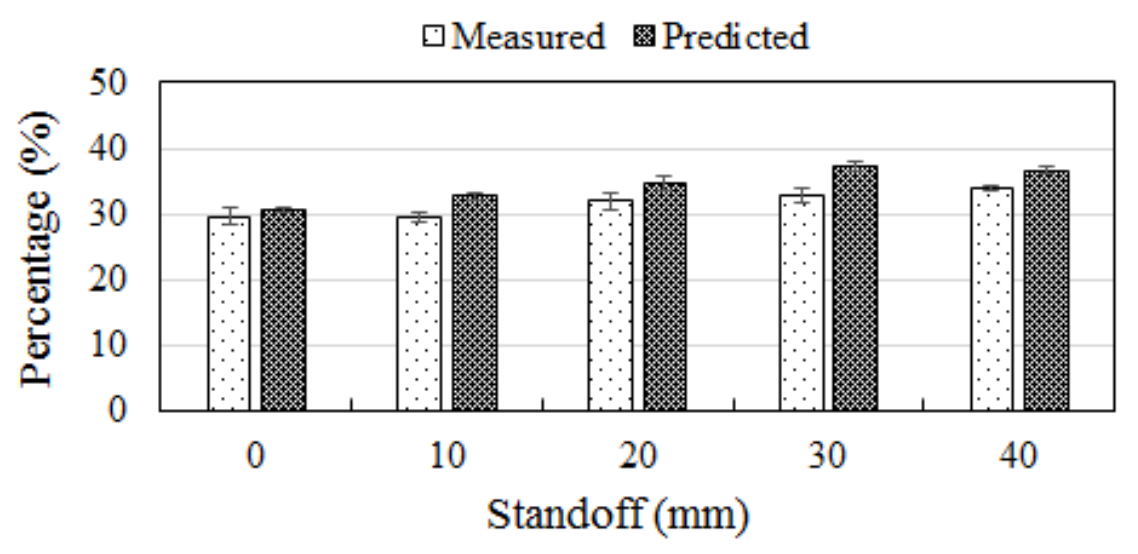

(a)

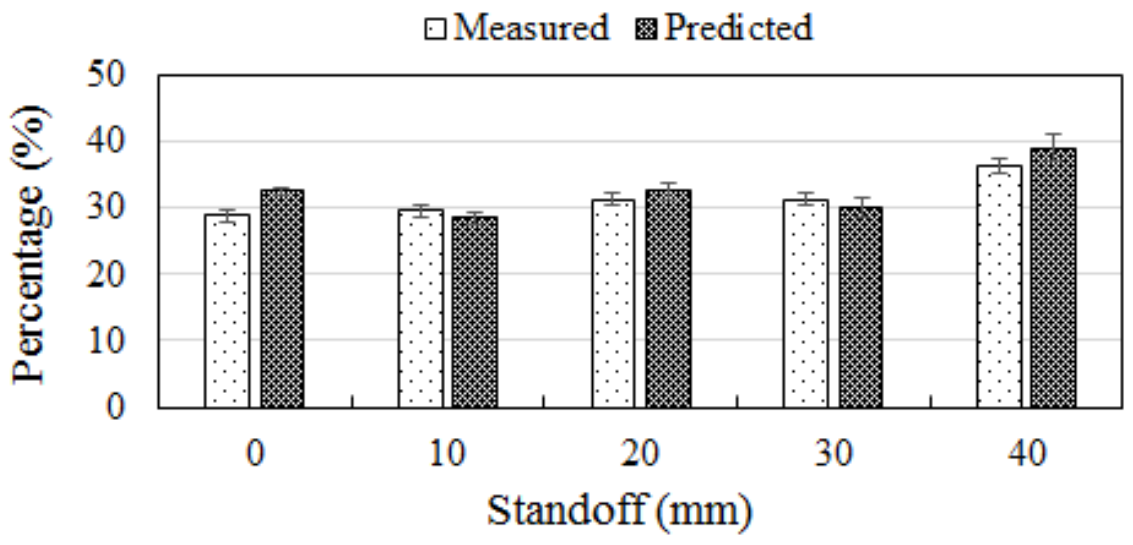

(b)

Figure 2.13 Predicted and measured percentages of oriented particles $\left(0^{\circ}-10^{\circ}\right)$ at different standoffs for (a) grit 90, and (b) grit 60 particles at $300 \mathrm{kPa}$. Scatter bars on predicted values show standard deviation of multiple runs of the model using 200 particles.

\subsection{Influence of particle orientation on particle embedding}

Since it was shown in Section 2.4 that increased standoff distances resulted in higher percentages of oriented particles, it was expected that the number of embedded particles also would increase with standoff. Indeed, Fig. 2.14 shows that the measured percentage of embedded 
particles from the experiments of Section 2.2.3 at $300 \mathrm{kPa}$ increased with standoff. This increase was statistically significant for any two standoffs with at least $20 \mathrm{~mm}$ difference (t-test, P value $<0.05$ ). For example, the percentage of the embedded particles for grit 60 abrasives utilizing 300 $\mathrm{kPa}$ jet pressure was 1.2 times higher at standoff of $40 \mathrm{~mm}$ than at $10 \mathrm{~mm}$. This increase was due to a $14.7 \%$ increase in the average kinetic energy of the particle and a $24 \%$ increase in the percentage of the oriented particles. Figure 2.14 also shows that the percentage of embedded particles at a typical standoff increased with particle size. For example, the percentage of embedded particles at a $30 \mathrm{~mm}$ standoff for grit 90 abrasives, was 1.25 times higher than that for grit 180 abrasives under same test conditions.

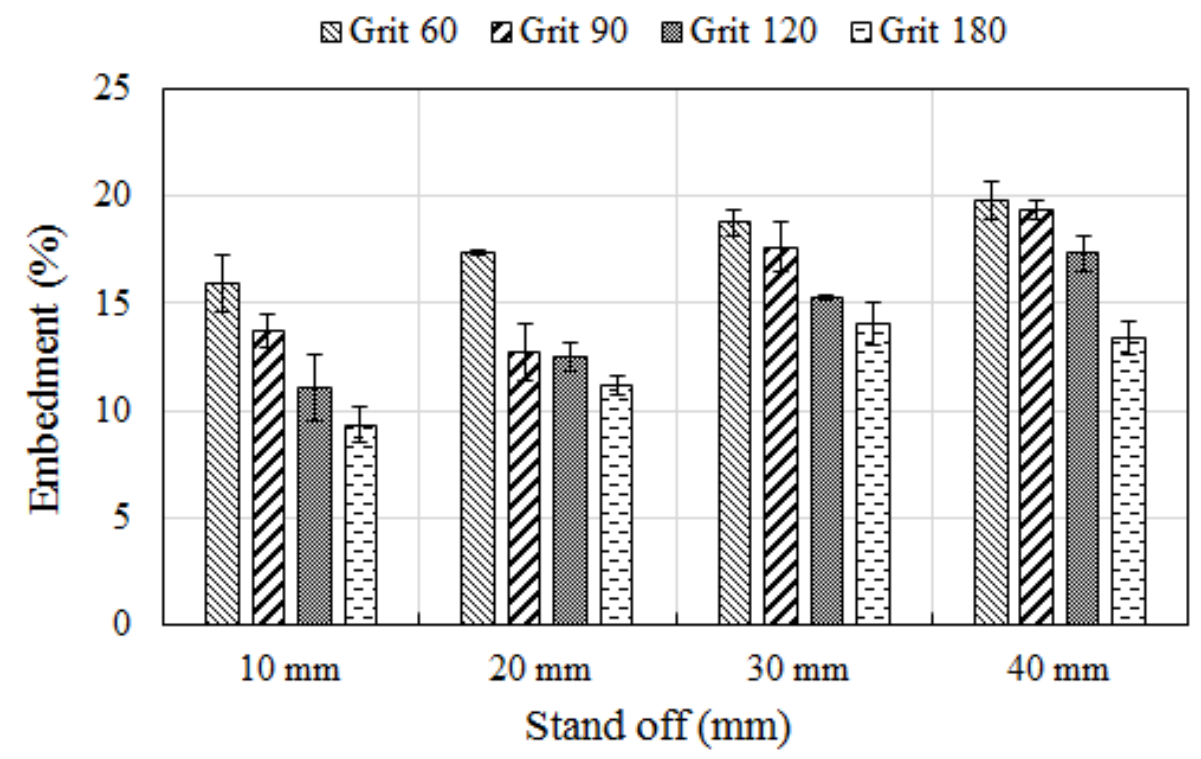

Figure 2.14 Percentage of embedded particles at different standoffs for different particles sizes at $300 \mathrm{kPa}$. Scatter bars show the standard deviation.

Figure 2.15 shows a correlation between the percentage of launched particles that embedded, and the percentage that were oriented (between $0^{\circ}$ and $10^{\circ}$ orientation angle) for all particle sizes and standoffs at a $300 \mathrm{kPa}$ pressure. The approximately linear correlation in Fig. 2.15 indicates that approximately $50 \%$ of the oriented particles actually embedded. 


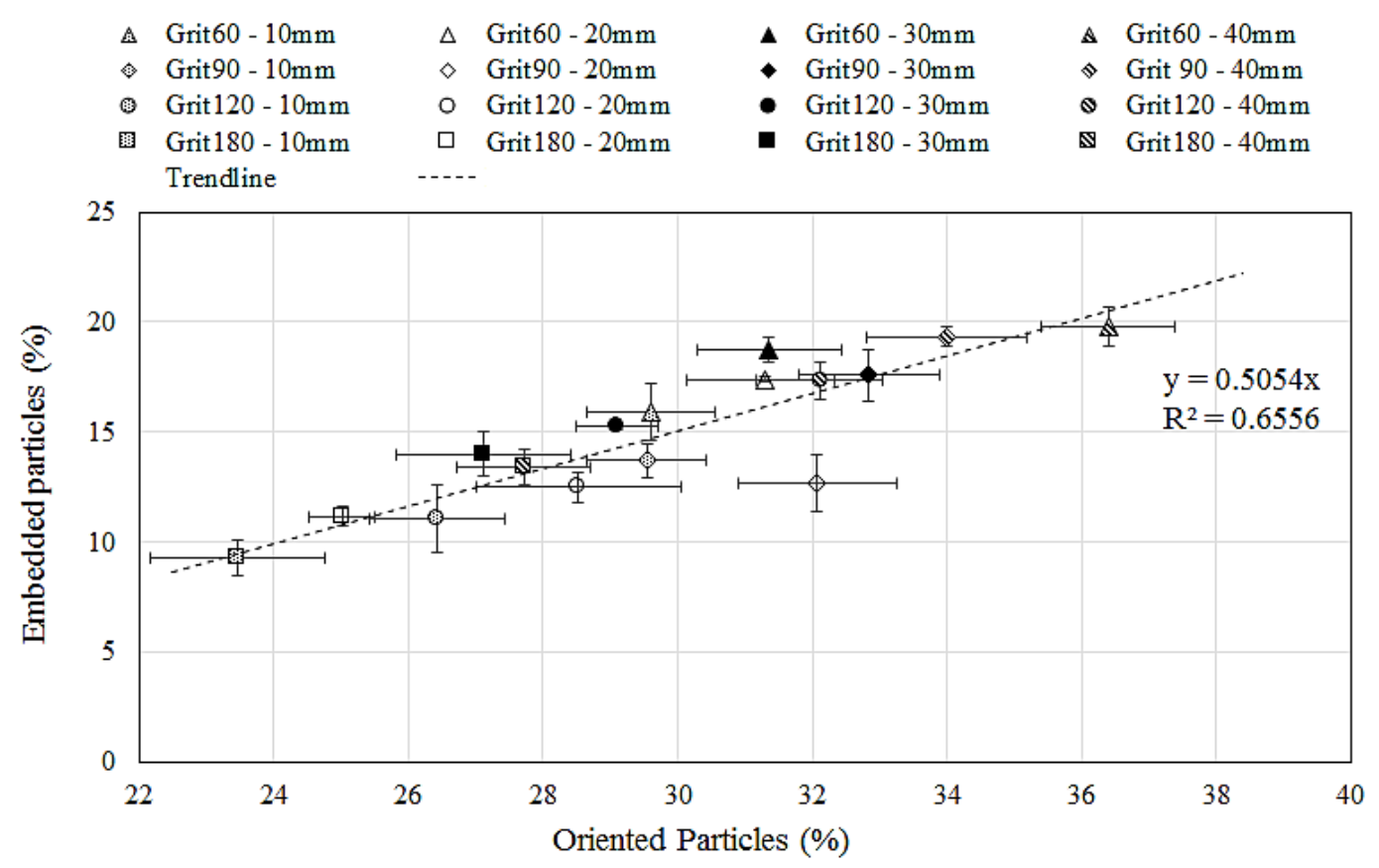

Figure 2.15 Correlation between percentage of embedded and oriented particles at $300 \mathrm{kPa}$. The dashed line indicates a linear fit to all data. Scatter bars show standard deviations.

The shortcoming of the above comparison, based on measurements made at different standoffs for a single pressure, is that the increase in standoff not only leads to a higher percentage of oriented particles, but also to a higher abrasive kinetic energy. Therefore, the correlation in Fig. 2.15 cannot be said to be due to orientation alone. Recalling that pressure had a negligible effect on orientation (Section 2.4.4), another set of experiments was performed in which the pressure was adjusted so that equal average particle velocity was obtained at two different standoffs. Figure 2.16 shows that, for the same particle velocity, the percentage of embedded particles at $40 \mathrm{~mm}$ standoff was 1.095 times higher than at $10 \mathrm{~mm}$, due entirely to the 1.15 times higher percentage of particle orientation at $40 \mathrm{~mm}$. It also shows that, even at a constant velocity, similar to the correlation in Fig. 2.15, around 56\% of oriented particles actually embed.

While these experiments are preliminary since they were performed on only a single material, should this correlation prove to be typical for a class of metals, or were there a method to estimate the correlation, then the demonstrated ability of the model to accurately predict instantaneous orientation suggests a procedure whereby estimates of embedding percentage could 
be obtained for a wide variety of applications. Measurement and modeling of such orientation/embedding correlations is a topic for continued study.

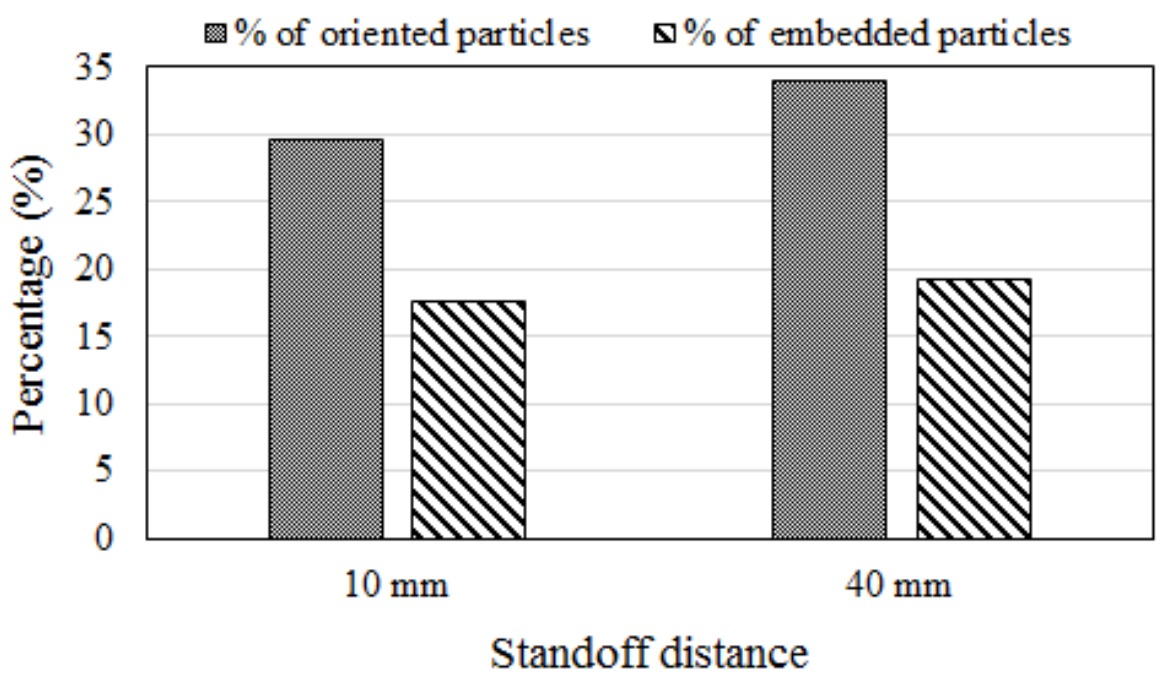

Figure 2.16 Comparison of percentage of oriented particles and embedded particles for the tests at $10 \mathrm{~mm}$ standoff and $500 \mathrm{kPa}$ pressure with $40 \mathrm{~mm}$ standoff and $300 \mathrm{kPa}$ (grit 90). In both cases, the average particle velocity was $\sim 135 \mathrm{~m} / \mathrm{s}$.

Getu et al. [16] modeled embedding by assuming randomly orientated idealized angular particles impacting polymeric targets described by a rigid-plastic material model. While the target is different, some useful conclusions can be drawn by comparison with their work. For example, they reported that there was a preferred orientation for particles to embed with a minimum impact velocity, i.e., at a given incident velocity, particles with preferred orientation were more likely to embed. Their conclusion is that preferred orientation occurs when the sum of impact angle and orientation angle is near $90^{\circ}$, which is consistent with the present study, which found that orientations between $0^{\circ}-10^{\circ}$ are likely to embed at a $90^{\circ}$ impact angle. Getu et al. [16] also reported that approximately $25 \%$ of $136 \mu \mathrm{m}$ garnet particles in their rigid-plastic model met a necessary, but not sufficient condition for embedment, i.e. the particles maintained contiguous contact with the target during impact. This compares well with the $28 \%$ of oriented grit 120 particles found in the present study at the same $20 \mathrm{~mm}$ standoff distance and similar pressures. The ratio between the embedded particles to those meeting this criteria in Getu et al.'s work [16] was 0.34, which is 
roughly comparable with the 0.42 ratio between the percentage of embedded to oriented grit 120 particles in the present study. While Getu et al. [16] did not establish whether particles meeting this first criteria were necessarily those which were oriented, the present results support that view. Finally, Getu et al. [16] also reported an insignificant influence of particle size on particle embedment in polymers; although the abrasive sizes utilized in that work were in a relatively narrow size range $(103$ and $136 \mu \mathrm{m})$. The present work is more in agreement with the experiments of ref. [98] in which a direct correlation between increasing particle size and increasing percentage of embedded particles was reported. A possible reason for this correlation could be the fragmentation of large particles upon initial or subsequent impacts that has been reported by, e.g., Walley and Field [15].

\subsection{Summary}

Double-pulsed laser shadowgraphy and image analysis were used to study the instantaneous orientation of abrasive particles within an air jet. The results of this study may find application in the optimization of abrasive jet machining process parameters, and in solid particle erosion studies. The main conclusions can be summarized as follows:

(i) At all pressures, between 26 and $37 \%$ of the particles were found to be oriented (orientation angle between $0^{\circ}-10^{\circ}$ ) with the jet axis.

(ii) There was a tendency for high aspect ratio particles to align with jet direction.

(iii) Although up to $\sim 30 \%$ of the particles were oriented with the jet axis at the nozzle exit, a statistically significant tendency was found for the likelihood of orientation to increase by up to 1.24 times at a distance of $40 \mathrm{~mm}$ from the jet exit.

(iv) The influence of pressure on particle orientation at a given standoff was found to be insignificant.

(v) Larger particles were more likely to orient themselves in the jet direction than smaller ones. At the same test condition (pressure and standoff), larger particles were more likely to embed. 
(vi) Although particles rotated rapidly, a large portion of them ( $\sim 64 \%)$ did not rotate for more than a full revolution over a $20 \mathrm{~mm}$ distance.

(vii) Orientation was correlated with embedding on virgin surfaces; i.e., approximately $50 \%$ of the oriented abrasives actually embedded. In terms of embedding, particle velocity was found to be much less important than orientation.

(viii) The model presented in this paper that took into account angular particle rotation in the calculation of instantaneous drag force was able to accurately predict measured particle linear velocity and orientation distribution for a wide variety of process conditions. The model may be used in the future to aid in the prediction of particle embedding for a wide variety of applications.

The measurements of instantaneous particle orientation from this chapter will be used in Chapter 3 as inputs for a three-dimensional numerical model that will allow the prediction of their propensity for embedment an $\mathrm{Al}$ 6061-T6 target. 


\section{Numerical Modeling of Particle Embedment during Solid Particle Erosion of Ductile Materials}

This chapter is based on the following published paper:

V. Hadavi, M. Papini, Numerical modeling of particle embedment during solid particle erosion of ductile materials, Wear 342-343 (2015) 310-321.

\subsection{Introduction}

Solid particle erosion involves the successive impact between particles and a surface that results in material removal $[1,2,3,8]$. It occurs in a wide variety of industrial processes including, amongst many others, abrasive jet machining, surface polishing, blast cleaning, and the erosion of gas pipelines and turbo machinery. When the particles impact relatively ductile surfaces, the potential for particle embedment exists, and this may greatly affect the resulting material removal

rate and surface quality. For example, in operations that utilize abrasive jets to purposefully remove target material such as in abrasive air, slurry and water jet micro-machining, embedded abrasives may reduce the erosion rate [10], negatively impact the heat transfer rate in abrasive jet micro-machined heat exchangers, and increase the roughness, thus affecting the flow in micromachined microfluidic devices $[10,11]$.

There have been a number of experimental works aimed at determining the parameters that affect particle embedment. These studies are sometimes contradictory. For example, for a limited range of sizes, Getu et al. [16] reported that particle size did not have a significant influence on particle embedment, while Day et al. [98] and Hadavi et al. [99] found that particle embedment increased with particle size. Getu et al. [16] also reported the existence of a minimum impact velocity required for particle embedment.

Temperature can significantly influence the mechanical properties and behaviour of several types of materials (e.g., polymers) [18,19,20,21,27], and therefore may also influence embedding. Getu et al. [21] conducted extensive investigations on the cryogenic abrasive jet micro-machining of polymers and found that, at all angles of attack and for all the tested materials, the amount of 
particle embedding was significantly reduced.

Particle impact angle and velocity also have important roles in the embedment of particles. Getu et al. [16] reported that the target area covered by embedded particles decreased when blasting at oblique impact angles [16]. They also observed that the minimum velocities that caused embedding occurred when the particle was oriented such that the incident velocity vector was aligned with the major axis of the particle $[16,99]$. This is most likely because such a configuration reduces the probability of rebound rotational energy being induced during the impact, as also noted by Papini and Spelt [30].

A number of investigators have identified an equilibrium phase when the number of embedded particles reaches a plateau. For instance, Getu et al. [16,21] reported that the number of embedded $\mathrm{Al}_{2} \mathrm{O}_{3}$ particles in polymer targets increased with increasing particle dose, until a critical dose had been reached. This is in agreement with $\mathrm{Wu}$ et al. [22], who observed that aluminium alloy specimens initially gained mass due to embedment of $\mathrm{SiC}$ and $\mathrm{Al}_{2} \mathrm{O}_{3}$ abrasives, and that a steady state was achieved only after long transients. Chen et al. [14] found a similar phenomenon when observing $\mathrm{Al}_{2} \mathrm{O}_{3}$ particle embedment into Hastelloy X. $\mathrm{Zu}$ et al. [100] also reported the presence of embedded silica abrasives in a pure aluminium target. The steady-state likely occurs when the rate of particle embedment reaches that of material removal [17].

The criteria for embedment of spherical and angular particles appears to be different [16]. Walley and Field [15], analyzed spherical particle impact craters and hypothesized that embedment occurs when a particle penetrates sufficiently to be surrounded by deformed material, preventing the elastic rebound forces from it. This is consistent with the work of Getu et al. [16], who reported that spherical particle embedment is a function of maximum penetration depth, which depends on the dynamic friction on the incident penetration trajectory. For angular particles, Getu et al. [16] suggested that the embedding criterion was more complex, depending more on the static friction at maximum penetration.

The present work aims to predict embedment of realistically-shaped angular particles into a relatively ductile metal target. In pioneering work with flat square plates, Hutchings [28] determined that initial particle orientation can strongly influence the erosion mechanisms of a ductile material, and developed a rigid-plastic model to predict the trajectory of such plates during impact. Getu et al. [16] utilized similar principles in developing the only existing angular particle embedment model, hypothesizing that impacting idealized rhomboid-shaped angular particles 
would embed into an elastic, perfectly-plastic polymeric material if two basic criteria were met. The first criterion was that the particle trajectory during impact would be such that a contiguous surface contact between the target and abrasive would be maintained during the impact, and the second was that the friction force that tended to keep the particle embedded would be larger than the elastic rebound force. Getu et al. [16] used a two-dimensional rigid-plastic target material model that assumed a constant contact pressure, in order to predict the trajectory of idealized rhomboid particles as they impacted, and thus whether contiguous contact was maintained. For cases where it did, they used an elastic rebound model and found that regardless of particle size and impact angle, the static friction coefficient remained approximately constant for a given particle-target system [16]. Despite its usefulness in providing a baseline to understand embedment, the model is inappropriate for use on metallic materials that strain and strain rate harden. Furthermore, rigid plastic models cannot predict thermal softening, crater edge pileup, and other phenomena such as non-uniform contact stresses that may affect particle kinematics and embedment.

Numerical methods can be used to address some of the shortcomings of rigid-plastic models in the analysis of solid particle erosion. Most previous finite element (FE) models utilized spherical particles that, compared to angular particles, induce a relatively small degree of plastic deformation into the target. For instance, Junkar et al. [101] applied FE to simulate the impact between single spherical particles and a substrate in water jet machining and predicted the crater depth. Shimizu et al. [102-103] used a plain-strain approximated FE model to investigate material removal rate and distribution of plastic strain in the extruded material in the front of an impacting spherical particle in mild steel, ferritic and spherical-graphite cast iron. El Tobgy and Elbestawi [104] developed FE models for the impact of spherical particles on Ti-6Al-4V alloy substrate and studied the effect of particle size and incident angle and velocity on removed material. They reported that steady state was achieved after 3 impacts and found their models to be in fair agreement with the analytical models of Finnie [105], Bitter [106] and Hashish [107]. Wang and Yang [108] used a Lagrangian FE technique to study the influence of incident angle and velocity on the erosion rate resulting from the impact of spherical particles on a Ti-6Al-4V alloy surface, and reported reasonable agreement with experiments performed using angular particles [109].

In reality, most solid particle erosion problems involve the impingement of angular particles that leave much larger amounts of plastic deformation on the substrate. Takaffoli and 
Papini [31] reported that distortion of finite elements in FE modeling of angular particle impacts resulted in inaccurate calculation of strain and stress and increased the computational time dramatically. They also reported that adaptive re-meshing and element deletion techniques could limit element distortion, but at the penalty of a large computational cost, or a large inaccuracy, respectively.

Mesh-free methods such as smoothed particle hydrodynamics (SPH) utilize particles to represent the computational domain that are not connected together, and therefore large deformation problems can be dealt with more effectively [110]. Takaffoli and Papini $[31,111,112]$ recently demonstrated that SPH methods can be used to successfully model single and multiple angular particle impacts in the erosion of metal targets by aluminum oxide particles. Their models utilized realistically shaped particles and considered strain and strain rate hardening, and were thus able to accurately predict measured particle kinematics, crater dimensions, material removal, and pile-up height. They did not, however, consider particle embedment in their models. They focused on dynamic frictional forces affecting the incident trajectory, rather than static ones affecting rebound or embedment.

In summary, there is currently no model that can be used to study the embedment of realistic particles into ductile metal targets. The only existing model, that of Getu et al. [16] for rhomboid particle impact on perfectly plastic polymers, revealed some fundamental aspects of the embedment process. However, it is unsuitable for detailed study of the embedment of more realistic particle geometries on strain and strain rate hardening metals. The aim of the present work was to determine whether SPH could be used with appropriate constitutive models and realistic angular particle geometries to predict embedment in such materials, and to shed more light on the effect of process parameters on the likelihood of embedment.

\subsection{Experiments}

\subsubsection{Measurement of particle embedment}

Experiments were conducted to determine percentage embedment, i.e. the percentage of the total number of impacting particles that remained embedded. Al 6061-T6 (90 BHN) targets were impacted by short bursts of grit 90 and grit 60 angular silicon carbide ( $\mathrm{SiC}$ ) abrasive, whose distribution of planar area, roundness, aspect ratio and circular diameter were measured in a previous study [10] using an optical particle sizing system (Clemex PSA Research Unit, Clemex 
Technologies Inc., Longueuil, Quebec, Canada). The average particle thickness was measured using a non-contact optical profilometer (Nanovea ST400, Micro Photonics Inc., Allentown, PA, USA). The average particle circular diameter and roundness are given in Table 3.1, and the detailed distributions of the other parameters can be found in Chapter 2. In all cases, the average circular diameter of the particles was approximately 3.4-3.8 times larger than their average thickness, indicating that the particles were relatively flat with a uniform thickness.

A micro-abrasive blaster (Accuflo, Comco Inc., Burbank, CA, USA) operating at air pressures of 100, 300 and $500 \mathrm{kPa}$ and fitted with a $1.5 \mathrm{~mm}$ diameter, $40 \mathrm{~mm}$ long nozzle was used to blast the particles. The $5.5 \mathrm{~mm}$ thick targets were placed at 20 and $40 \mathrm{~mm}$ standoff distance from the nozzle exit, and the jet impacted perpendicular $\left(90^{\circ}\right)$ to the target, and at three oblique incidences $\left(30^{\circ}, 45^{\circ}\right.$ and $\left.60^{\circ}\right)$. The mass flow rate was set sufficiently low $(1.8-2.4 \mathrm{~g} / \mathrm{min})$ to ensure minimal particle to particle interaction. The targets were polished to a roughness of $0.02 \mu \mathrm{m}$ and the jet was directed through a programmable shutter device having an opening width of $5 \mathrm{~mm}$ operating at a linear velocity of $0.4 \mathrm{~m} / \mathrm{s}$, in order to limit the number of particles impacting the surface. The target was exposed to the jet of particles for approximately $12.5 \mathrm{~ms}$, sufficient to ensure non-overlapping impacts so that individual impact craters and embedded particles could be counted. Table 3.1 summarizes the process parameters used in the experiments and simulations.

Table 3.1 Process parameters for embedding experiments and numerical simulations.

\begin{tabular}{|c|c|c|c|c|c|c|}
\hline $\begin{array}{c}\text { Grit } \\
\text { Size }\end{array}$ & $\begin{array}{c}\text { Average } \\
\text { Circular } \\
\text { Diameter } \\
(\mu m)[99]\end{array}$ & $\begin{array}{c}\text { Roundness } \\
{[99]}\end{array}$ & $\begin{array}{c}\text { Pressure } \\
(\mathrm{kPa})\end{array}$ & $\begin{array}{c}\text { Average } \\
\text { Velocity } \\
(\mathrm{m} / \mathrm{s})[99]\end{array}$ & $\begin{array}{c}\text { Impact Angles } \\
(\mathrm{deg} .)\end{array}$ & $\begin{array}{c}\text { Standoff } \\
\text { Distance }(\mathrm{mm})\end{array}$ \\
\hline 90 & 219 & 0.59 & 160 & 87 & 90 & 20 \\
\hline 90 & 219 & 0.59 & 300 & 120 & $30,45,60,90$ & 20 \\
\hline 60 & 363 & 0.60 & 100 & 39 & 90 & 40 \\
\hline 60 & 363 & 0.60 & 300 & 87 & 45,90 & 20 \\
\hline 60 & 363 & 0.6 & 500 & 115 & 90 & 40 \\
\hline
\end{tabular}

The blasted surfaces were cleaned using distilled water and dried using compressed air, and the blasted footprints were analyzed using scanning electron micrographs (SEM) to count the 
number of impact craters, and energy dispersive x-ray spectroscopy (EDX) to count the embedded particles, based on detection of $\mathrm{Si}$ in the craters (Fig.3.1). The number of incident particles was assumed to be equal to the sum of the impact crater and embedded particle sites. The incident number of particles was also calculated based on the dimensions of an average particle and the particle mass flow rate. For example, given that the average area, thickness and density of the utilized particles with $D_{\text {circular }}=219.2 \mu \mathrm{m}$ were $38909 \mu \mathrm{m}^{2}, 64.7 \mu \mathrm{m}$ and $3100 \mathrm{~kg} / \mathrm{m}^{3}$ respectively, the average mass of one particle was calculated to be $7.937 \times 10^{-3} \mathrm{mg}$. Based on a measured mass flow rate at $300 \mathrm{kPa}$ of $1.76 \mathrm{~g} / \mathrm{min}$, this yields a calculated value of 46 particles for the $12.5 \mathrm{~ms}$ exposure time, which agreed well with the 41 obtained by counting impact and embedded sites on the blasted surface. Figure 3.1 shows the SEM and EDS image of some of the SiC particles embedded in an Al 6061-T6 target. Each experiment was repeated 3 times.

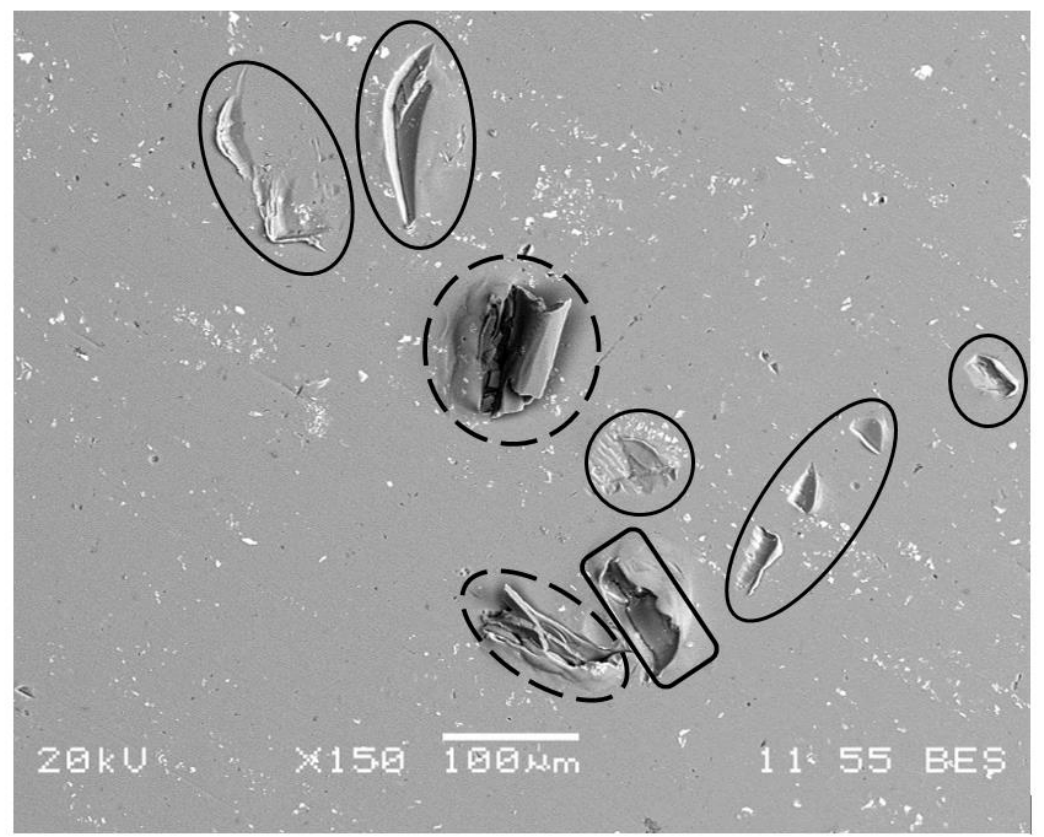

(a) 


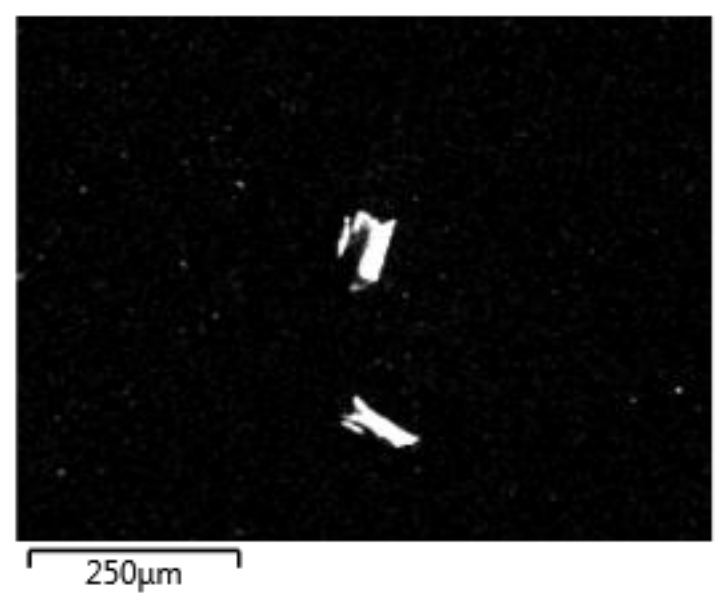

(b)

Figure 3.1 Images of typical craters with (dashed lines) and without (solid lines) embedded SiC particles using (a) SEM; (b) EDS with white indicating presence of Si.

\subsubsection{Measurement of particle orientation and velocity}

Since previous work [16] indicated that the likelihood of embedment depended strongly on particle orientation upon impact and, to a lesser extent, on the incident particle velocity, measurements of these quantities were required as inputs for the models of Section 3.3.3. Distribution of particle orientations and velocities within an abrasive jet under operating conditions identical to those presently utilized was previously measured in Chapter 2. Briefly, high resolution images of the abrasives in flight were obtained using a pulsed laser shadow-graphic technique, explained in detail in [95], and analyzed using PSA Professional Research Particle Size and Shape Image Analysis software (Clemex Technologies Inc., Longueuil, Quebec). The orientation of the particles was defined as the angle between the line connecting center of mass and most downstream vertex of particle, as shown in Fig. 3.2. 


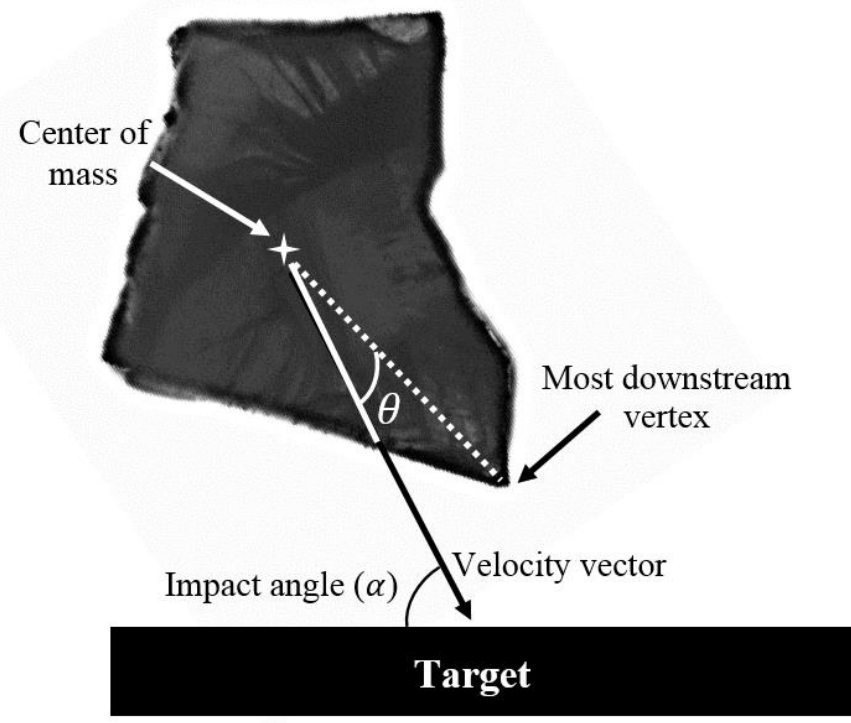

Figure 3.2 Orientation angle $(\theta)$ of an abrasive particle in the jet.

As an example, the distribution of the measured orientation angles of grit $90 \mathrm{SiC}$ particles at $20 \mathrm{~mm}$ standoff and $300 \mathrm{kPa}$ is shown in Fig. 3.3. The average velocity under these conditions was measured as $120 \mathrm{~m} / \mathrm{s}$. Approximately $32 \%$ of the grit 90 particles blasted at $300 \mathrm{kPa}$ had an orientation angle between $0^{\circ}-10^{\circ}$ at a $20 \mathrm{~mm}$ standoff. SEM analysis of the impacted target showed that the majority of particles impacted the target perpendicular to their thickness. The distributions of orientation (not shown) and average velocity (Table 3.1) at the other standoffs and pressures, and for the grit 60 abrasives are also taken from Chapter 2. The use of these distributions in the simulation is explained in Section 3.3.3. 


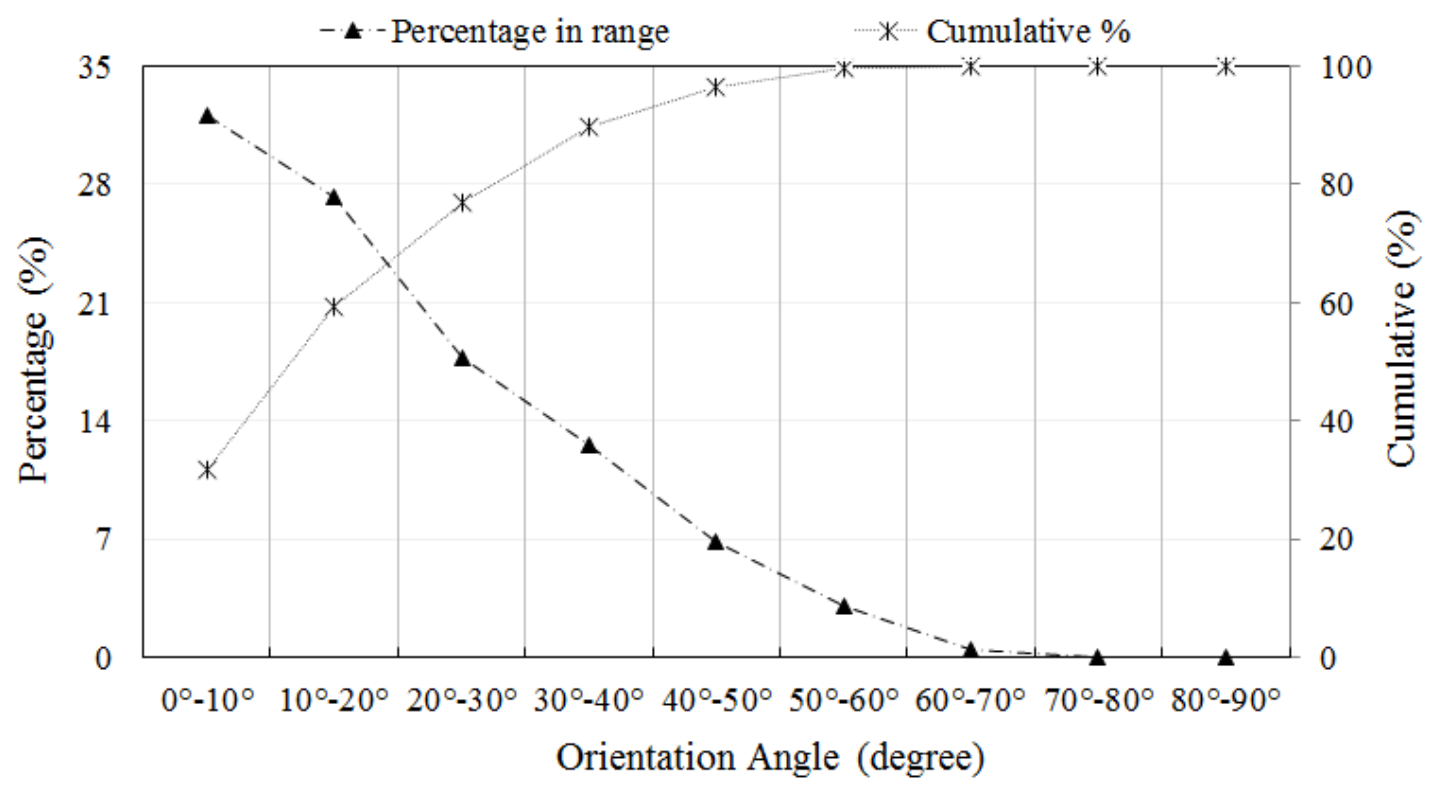

Figure 3.3 Distribution of orientation angles at a $20 \mathrm{~mm}$ standoff distance from the nozzle. Grit 90 particles at $300 \mathrm{kPa}$ jet pressure [99]. The lines are only to guide the eye.

\subsection{Numerical simulations}

Numerical models were implemented using LS-Dyna Version 971 (Livermore Software Technology Corporation, Livermore, CA, USA), to simulate the embedment of a number of single non-overlapping SiC particles in an A16061-T6 target, consistent with the experiments of Section 3.2.1, which ensured that no point on the target was impacted by more than one particle. The use of various target surface areas, thicknesses and distances between SPH particles was investigated in order to compromise between computational time and accuracy. It was found that for target surface areas larger than $300 \mu \mathrm{m} \times 300 \mu \mathrm{m}$, the influence of edge of the target on the resulting stresses and deformations could be ignored. The influence of the thickness of target was found to be insignificant when the thickness was larger than 150-200 $\mu \mathrm{m}$. Therefore, the target was modeled as a $300 \mu m \times 300 \mu m \times 200 \mu m$ box. Decreasing the SPH distance in the range 2-5 $\mu m$ for this target size resulted in an exponential increase in the computational time, while the rebound velocity only changed by $2 \%$. Therefore, a $5 \mu \mathrm{m}$ spacing was utilized in all further modeling, in agreement with SPH spacing used by Takaffoli and Papini [111-112] for similarly sized impacting particles of a different type. As recommended in previous studies [113], uniform particle spacing was used in the present models rather than a biased SPH model with a finer spacing at the impact site. 
Preliminary simulations showed that the influence of boundary conditions at the target free surfaces on the particle rebound velocity and likelihood of embedding was insignificant. For a typical rebound phase of particles that took an average $0.5 \mu \mathrm{s}$, the induced stress wave travelling at $5240 \mathrm{~m} / \mathrm{s}$ in Al6061T6 traversed the target thickness $\sim 15$ times. Therefore, fixed rotation and displacement conditions were assigned to the SPH nodes at the target walls and bottom.

The impacting abrasive particles were modeled using three dimensional finite element representations of the geometries of the actual particles used in the experiments, as described in Section 3.3.3.1.

\subsubsection{Modeling of target}

\subsubsection{SPH theory}

Particles without fixed connectivity are used in SPH to discretize the domain of the problem. The material properties are attributed to the SPH particles which can also move relative to each other according to the governing conservation equations. The SPH formulation is based on the integral approximation or kernel approximation of a field function $f$ with a function $\langle f\rangle$ introduced by [110]:

$$
\langle f(\boldsymbol{x})\rangle=\int_{\Omega} f\left(\boldsymbol{x}^{\prime}\right) \cdot W\left(\boldsymbol{x}-\boldsymbol{x}^{\prime}, l\right) d \boldsymbol{x}^{\prime}
$$

where $W$ is the smoothing kernel function with the influence domain equal to a sphere with a radius of $2 l$ [113], and $\Omega$ is the domain of integration. The location of all the points inside the influence domain is determined by the vector $\boldsymbol{x}^{\prime}$. Consequently, the integration remains localized over the influence domain of the smoothing function when the value of the field function is calculated at a specific $\boldsymbol{x}$. A "particle approximation" of the field functions is defined as the discretized summation that represents the continuous integral of eq. (3.1). This conversion can be performed for any type of particle distribution over the influence domain. In other words, the particle approximation for $\langle f(\boldsymbol{x})\rangle$, assuming $N$ particles in the influence domain of the smoothing function $W$, is [110]:

$$
\left\langle f\left(\boldsymbol{x}_{i}\right)\right\rangle=\sum_{j=1}^{N} \frac{m_{j}}{\rho_{j}} f\left(\boldsymbol{x}_{i}\right) W\left(\boldsymbol{x}_{i}-\boldsymbol{x}_{j}, l\right)
$$


where $m_{j}$ and $\rho_{j}$ are the mass and density of the particles $j(=1,2,3, \ldots, N)$, respectively; and $\boldsymbol{x}_{i}$ indicates the location of particle $i$. Conservation equations for mass, moment and energy are also derived from particle approximations and are considered as the applicable terms for inter-particle forces [110], i.e.,

Conservation of Mass: $\frac{\partial \rho_{i}}{\partial t}=\sum_{j=1}^{N} m_{j} v_{i j} \frac{\partial W_{i j}}{\partial x_{i}}$

Conservation of Momentum: $\frac{\partial v_{i}}{\partial t}=\sum_{j=1}^{N} m_{j}\left(\frac{\sigma_{i}}{\rho_{i}^{2}}+\frac{\sigma_{j}}{\rho_{j}^{2}}\right) \frac{\partial W_{i j}}{\partial x_{i}}$

Conservation of Energy: $\frac{\partial u_{i}}{\partial t}=\sum_{j=1}^{N} m_{j}\left(\frac{\sigma_{i} \sigma_{j}}{\rho_{i} \rho_{j}}\right) v_{i j} \frac{\partial W_{i j}}{\partial x_{i}}$

$W_{i j}=W\left(\boldsymbol{x}_{j}-\boldsymbol{x}_{i}, l\right)$, and $\sigma_{i}$ and $\sigma_{j}$ are components of the stress tensor for particles $i$ and $j$, respectively. $v_{i j}$ is the relative velocity vector between particle $i$ and $j$.

The above conservation equations introduce inaccuracies for particles located at boundaries which lack neighbors. To address this, the renormalization formulation proposed in [32,113] was implemented. Local clustering of particles under tensile loads can also lead to instabilities in SPH simulations which may result in generation of unexpected fragments of the SPH target $[62,114]$. Plots of energy exchanges during a simulation can be used to detect the presence of such instabilities, usually characterized by the generation of artificial energy [110]. For the present models, such plots showed that the artificial energy generated in all cases was less than $5 \%$.

\subsubsection{Constitutive equation}

The Johnson-Cook material model in LS-Dyna, shown to be appropriate in previous SPH modeling of solid particle erosion processes that did not consider embedding [32], was used to represent the A16061-T6 target material in the present work. The Johnson-Cook model relates the magnitude of instantaneous yield stress, $\sigma_{y}$, to static yield stress, $\sigma_{0}$, the effective plastic strain rate, $\dot{\varepsilon}_{e f f}^{p}$, effective plastic strain $\varepsilon_{e f f}^{p}$ and the temperature, $T$ [115] as

$$
\sigma_{y}=\left(\sigma_{0}+B\left(\varepsilon_{e f f}^{p}\right)^{n}\right)\left(1+\operatorname{cln} \dot{\varepsilon}^{*}\right)\left(1-T^{* m}\right)
$$


where $\mathrm{c}$ and $m$ characterize the strain rate and thermal softening of the material, respectively, and $B$ and $n$ the strain hardening at a reference strain rate, $\dot{\varepsilon}_{0}$, and reference temperature, $T_{r e f}$. The values of these parameters are taken from previous studies in the literature [116] for Split Hopkinson bar tests on A16061-T6, and are shown in Table 3.2. The normalized temperature $\left(T^{*}\right)$ and strain rate $\left(\dot{\varepsilon}^{*}\right)$ are

$$
\begin{aligned}
T^{*} & =\frac{T_{\text {inst }}-T_{\text {ref }}}{T_{\text {melt }}-T_{\text {ref }}} \\
\dot{\varepsilon}^{*} & =\frac{\dot{\varepsilon}_{\text {eff }}^{p}}{\dot{\varepsilon}_{0}}
\end{aligned}
$$

where $\dot{\varepsilon}_{0}$ and $T_{\text {ref }}$ were considered to be $1 \mathrm{~s}^{-1}$ and $292 \mathrm{~K}$.

\begin{tabular}{|c|c|c|c|}
\hline Material & Parameter & Value & Unit \\
\hline \multirow{12}{*}{$\begin{array}{l}0 \\
\frac{1}{1} \\
\frac{1}{8} \\
8 \\
\frac{8}{2}\end{array}$} & Density, $\rho$ & 2800 & $\frac{\mathrm{kg}}{\mathrm{m}^{3}}$ \\
\hline & Poisson Ratio, $(v)$ & 0.33 & \\
\hline & Shear Modulus (G) & 26 & $G P a$ \\
\hline & Melting Temp., $T_{m}$ & 925 & $K$ \\
\hline & Specific heat, $C_{p}$ & 885 & $\mathrm{~J} / \mathrm{kg} \mathrm{K}$ \\
\hline & $\sigma_{0}[116]$ & 335 & $M P a$ \\
\hline & $\mathrm{B}[116]$ & 85 & $M P a$ \\
\hline & c [116] & 0.012 & \\
\hline & $\mathrm{n}[116]$ & 0.11 & \\
\hline & $\mathrm{m}[116]$ & 1 & \\
\hline & $\begin{array}{c}\text { D1, D2,D3,D4,D5 } \\
{[117]}\end{array}$ & $-0.77,1.45,0.47,0,1.6$ & \\
\hline & $\begin{array}{l}\text { Bulk Speed of Sound } \\
\text { (C) }[114]\end{array}$ & 5240 & $\mathrm{~m} / \mathrm{s}$ \\
\hline
\end{tabular}

Table 3.2 Properties of $\mathrm{Al} 6061 \mathrm{~T} 6$ target and $\mathrm{SiC}$ abrasive particles. 


\begin{tabular}{|c|c|c|c|}
\hline \multirow{4}{*}{} & $S_{1}, S_{2}, S_{3}[114]$ & $1.4,0,0$ & \\
\cline { 2 - 4 } & $\begin{array}{c}\text { Gruneisen Constant, } \gamma \\
{[114]}\end{array}$ & 1.97 & \\
\cline { 2 - 4 } & $\bar{\alpha}[114]$ & 0.48 & $\mathrm{~s}^{-1}$ \\
\cline { 2 - 4 } & $\mathrm{D}[118]$ & 6500 & $\mathrm{HV}$ \\
\cline { 2 - 4 } & $\mathrm{q}[118]$ & 4 & $\frac{\mathrm{kg}}{\mathrm{m}^{3}}$ \\
\hline & Hardness & 107 & $\mathrm{GPa}$ \\
\hline \multirow{4}{*}{$=$} & Density & 3100 & $\mathrm{HV}$ \\
\cline { 2 - 4 } & Young Modulus & 410 & \\
\cline { 2 - 4 } & Poisson Ratio $(v)$ & 0.14 & \\
\cline { 2 - 4 } & Hardness & 2600 & \\
\cline { 2 - 4 } & & & \\
\hline
\end{tabular}

The second term in the right hand side of equation (3.6) reflects the strain rate sensitivity which becomes significant for $\mathrm{Al}$ 6061-T6 at strain rates higher than $10^{3}\left(\mathrm{~s}^{-1}\right)$ [117]. For solid particle erosion simulations, the Cowper-Symonds equation which relates the yield stress in a quasi-static condition to the magnitude of yield stress in dynamic loading has been found to provide a better fit to experimental data than the Johnson-Cook [118]:

$$
\frac{\sigma_{e f f}}{\sigma_{\text {Static }}}=1+\left(\frac{\dot{\varepsilon}_{e f f}}{D}\right)^{\frac{1}{q}}
$$

where $\sigma_{e f f}, \sigma_{\text {Static }}$ and $\dot{\varepsilon}_{e f f}$ are the effective stress, uniaxial yield strength (static) and effective strain rate, respectively. Thus strain rate hardening following eq. (3.9) was implemented in LSDyna instead of that appearing in the second term of eq. (3.6). The empirical constants $D$ and $q$ (Table 3.2) were based on those used in Ref. [118].

\subsubsection{Damage model}

The Johnson-Cook damage model was utilized to assess the accumulation of damage during the impact. Failure was assumed to occur when the damage parameter $F[119,120]$ : 


$$
F=\frac{\sum \Delta \varepsilon_{e f f}^{p}}{\varepsilon^{f}}
$$

reached a value of 1 , after which the stress in the SPH particles were set to zero and the induced stress was shared by neighboring particles. The failure strain $\varepsilon^{f}$ was defined as a function of stress triaxiality (the ratio of hydrostatic stress, $p$, to effective stress $\sigma_{e f f}$ ), strain rate and temperature as $[119,120]$

$$
\varepsilon^{f}=\left(D_{1}+D_{2} \exp \left[D_{3} \frac{p}{\sigma_{e f f}}\right]\right)\left(1+D_{4} \ln \dot{\varepsilon}\right)\left(1+D_{5} T^{*}\right)
$$

The values of $D_{i}$ for Al6061-T6 are taken from the literature [117] and given in Table 3.2.

\subsubsection{Equation of state}

The equation of state (EOS), required by the Johnson-Cook material model in LS-Dyna, relates the pressure to the changes in the volume of the material. The Mie-Gruneisen EOS was used to define the pressure, $P$, for the compressed material as follows [120]:

$$
P=\frac{\rho_{0} C^{2} \bar{\mu}\left(1+\left(1-\left(\frac{\gamma}{2}\right)\right) \bar{\mu}-\left(\frac{\bar{\alpha}}{2}\right) \bar{\mu}^{2}\right)}{\left(\left(1-\left(S_{1}-1\right) \bar{\mu}\right)-S_{2}\left(\frac{\bar{\mu}^{2}}{(\bar{\mu}+1)}\right)-\left(\frac{S_{3} \bar{\mu}^{3}}{(\bar{\mu}+1)^{2}}\right)\right)^{2}}+(\gamma+\bar{\alpha} \bar{\mu}) E_{0}
$$

and for expanded material as [120]:

$P=\rho_{0} C^{2} \bar{\mu}+\left(\gamma_{0}+\bar{\alpha} \bar{\mu}\right) E$

where $S_{1}, S_{2}, S_{3}$ and $C$ are unitless coefficients of, respectively, the slope and intercept of the shock velocity $\left(V_{s}\right)$-particle velocity curve $\left(V_{p}\right), \gamma$ is the unitless Gruneisen factor, $E_{0}$ is the internal

energy, and $\bar{\alpha}$ is a volume correction factor. The compression factor $\bar{\mu}=\frac{\rho}{\rho_{0}}-1$ is based on $\rho$ and 
$\rho_{0}$, the current and initial densities, respectively. The required constants for the Gruneisen EOS are available for Al 6061-T6 [114].

\subsubsection{Contact and friction}

As mentioned in Section 3.3, the abrasive particles were modeled using finite elements, and the target using SPH particles. As described by Takaffoli and Papini [111,112], the Automatic_Node_To_Surface contact option in LS-Dyna is the most appropriate algorithm for the contact between the FE particles and SPH target. Previous work on polymers indicated that particle embedment strongly depended on the static friction coefficient upon particle rebound [16]. The temperature rise induced by impacts of similarly sized particles on the same aluminum target used in the present study has been previously reported as significantly below the melting point of the aluminum alloy target [121]. Therefore, the friction coefficient was approximated as independent of temperature. The dry coulomb friction formulation of LS-Dyna uses an exponential interpolation function to smooth the transition between the static and dynamic friction coefficient so that its effective value is a function of relative velocity [120]. Given that the dynamic and static coefficients of friction are relatively close to each other for many contacting material pairs [120], for simplicity, they were considered equal in the present work.

Direct measurement of the static friction coefficient for such small angular particles on the target is challenging, if not impossible. Therefore, the friction coefficient was treated as a free parameter to be adjusted for a best fit of predicted particle embedding coverage to that measured in experiments performed using one particle size and velocity at perpendicular incidence. As will be discussed in Section 3.4.2, the friction coefficient determined in this manner was found to be within reasonable physical bounds. The performance of the model was then evaluated by using this friction coefficient to predict embedment using other angles of incidence, particle sizes, and velocities, and comparing to measured results (Section 3.4.2).

\subsubsection{Modeling of abrasive particles}

\subsubsection{Generation of 3D particle geometries}

The algorithm introduced by Takaffoli and Papini [111] was used to generate realistic 3D geometries of the actual $\mathrm{SiC}$ particles used in the experiments of Section 3.2.1, based on measurements of their planar area, circular diameter, thickness, and roundness distributions from 
Chapter 2. The profilometry measurements of Section 3.2.1 revealed that that the thickness and area were not correlated; therefore, a random value from the measured average particle thickness distribution was assigned to each particle. The area generated by the four vertices was extruded equal to the thickness to generate the CAD representation of the abrasives. Figure 3.4 shows the 2D projection of the generated particles and a sample of the real particles. It is noted that some of the particles impacted the target a second time during the rebound phase leaving secondary craters, as was also reported in refs. [31, 111]. Since this behavior had very little influence on the likelihood of particle embedment, i.e. the particles were far more likely to embed on the first impact, it was not considered further in the present work.

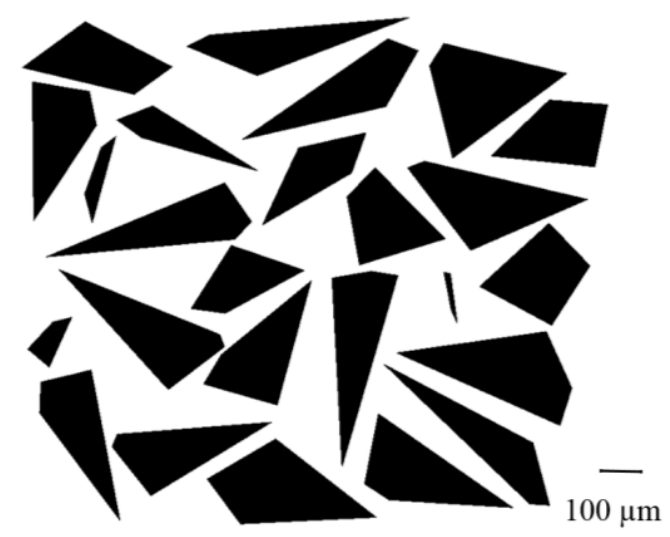

(a)

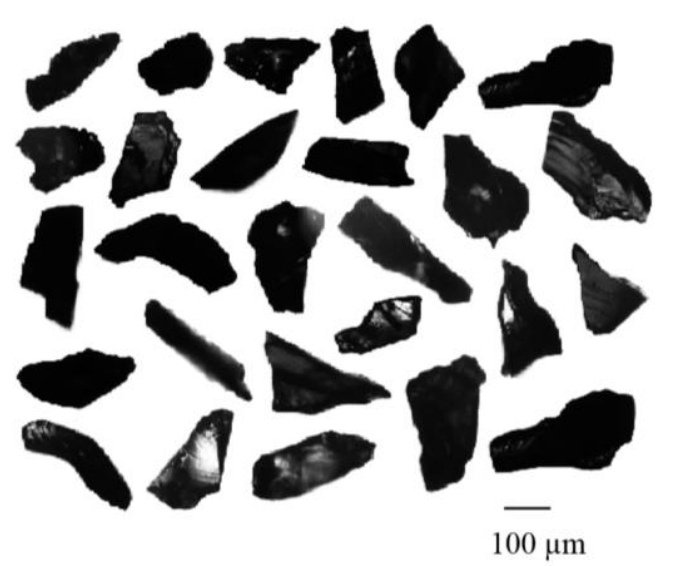

(b)

Figure 3.4 Geometry of a random sample of: (a) modeled particles and (b) actual SiC particles. 


\subsubsection{Constitutive equation and meshing of abrasive particles}

Since the hardness of $\mathrm{SiC}$ particles $(2600 \mathrm{HV})$ is much greater than the Al6061-T6 target material (107 HV), the particles were modeled using a rigid (non-deforming) material model. In order to reduce the computational time, each particle was modeled using a single solid element. The following image shows a typical mesh and geometry used in modeling a single impact. Using an Intel quad-core Core ${ }^{\mathrm{TM}} \mathrm{i} 7-3770 \mathrm{CPU}$ with $12 \mathrm{~GB}$ of RAM, it took approximately 20 mins to complete a typical impact simulation of $0.002 \mathrm{~ms}$. For each process condition in Table 3.1, consistent with the 48-109 impact sites of the experiments in Section 3.2.1, 100 particle impacts were modeled, each with an orientation assigned based on the measured distribution described in Section 3.2.2, and with the measured average velocity given in Table 3.1. The algorithm developed by Takaffoli and Papini [111] was modified so that center of mass and most downstream vertex of the particle (Fig. 3.2) could be detected and the angle between the lines connecting these two points (major axis) and the velocity vector calculated. Each modeled particle was then rotated by the aforementioned angle to obtain the prescribed orientation. Figure 3.5 illustrates a typical model.

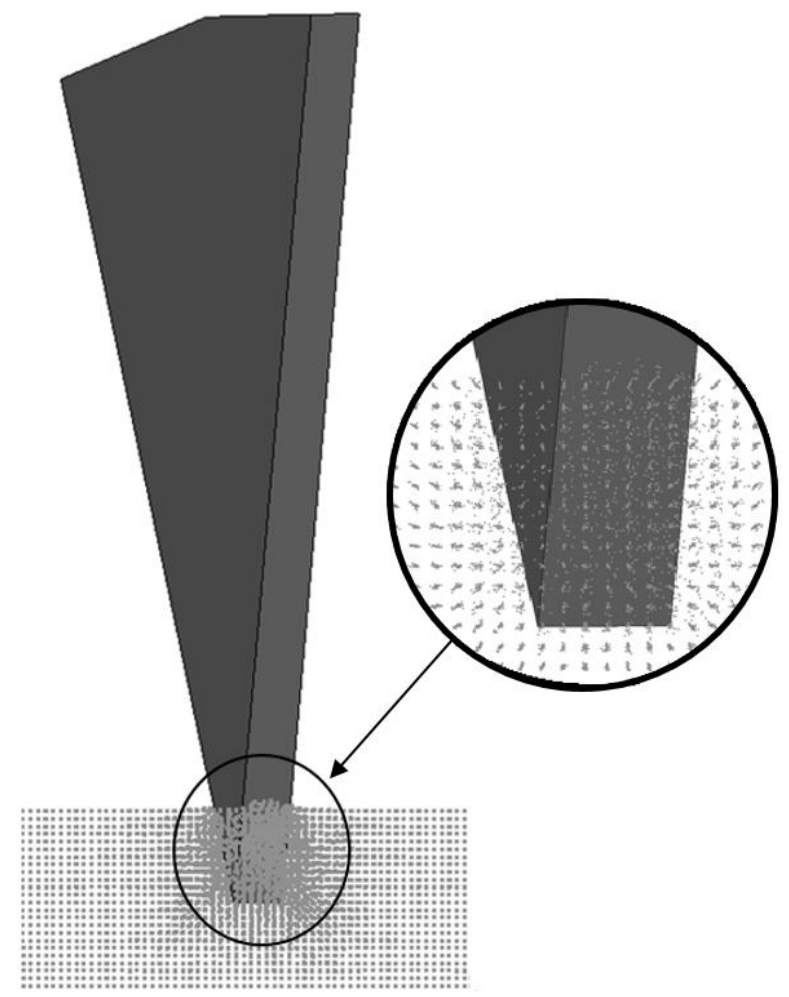

Figure 3.5 3D view of atypical single particle impact model after embedment. 


\subsection{Results and discussion}

As mentioned previously, the value of the friction coefficient between the $\mathrm{SiC}$ particles and the Al6061-T6 target was determined by calibrating the model such that the measured and predicted embedment percentage matched under one set of conditions (perpendicular incidence, $120 \mathrm{~m} / \mathrm{s}$, grit 90). As expected, preliminary SPH modelling indicated that particles striking the target on a blunt face rebounded even for very high values of friction coefficient. However, particles that struck the target with a sharp edge oriented to their velocity vector (jet axis) were predicted to embed if an appropriate value of friction coefficient was used.

\subsubsection{Determination of embedment in numerical models}

Figure 3.6 shows the dependence of the predicted velocity versus time curves on the friction coefficient (range: 0-2.5) used for a typical simulation of a single particle impact at normal incidence. It is noted that the resultant center of mass velocity need not reach zero at the maximum penetration depth (when time $\sim 0.55-0.8 \mu s$ ) due to induced rotations about the contact point. Despite this, particles may nevertheless embed. As expected, the rebound velocity decreased with increasing friction coefficient as the friction forces due to the clamping of the material surrounding the particle increased. A particle was considered to be embedded if the predicted rebound particle velocity approached zero as shown in the bottom curve of Fig. 3.6. As shown in Fig. 3.7a, the embedment was confirmed by the presence of the small final contact force which was zero for the rebounding particle of Fig. 3.7b. 


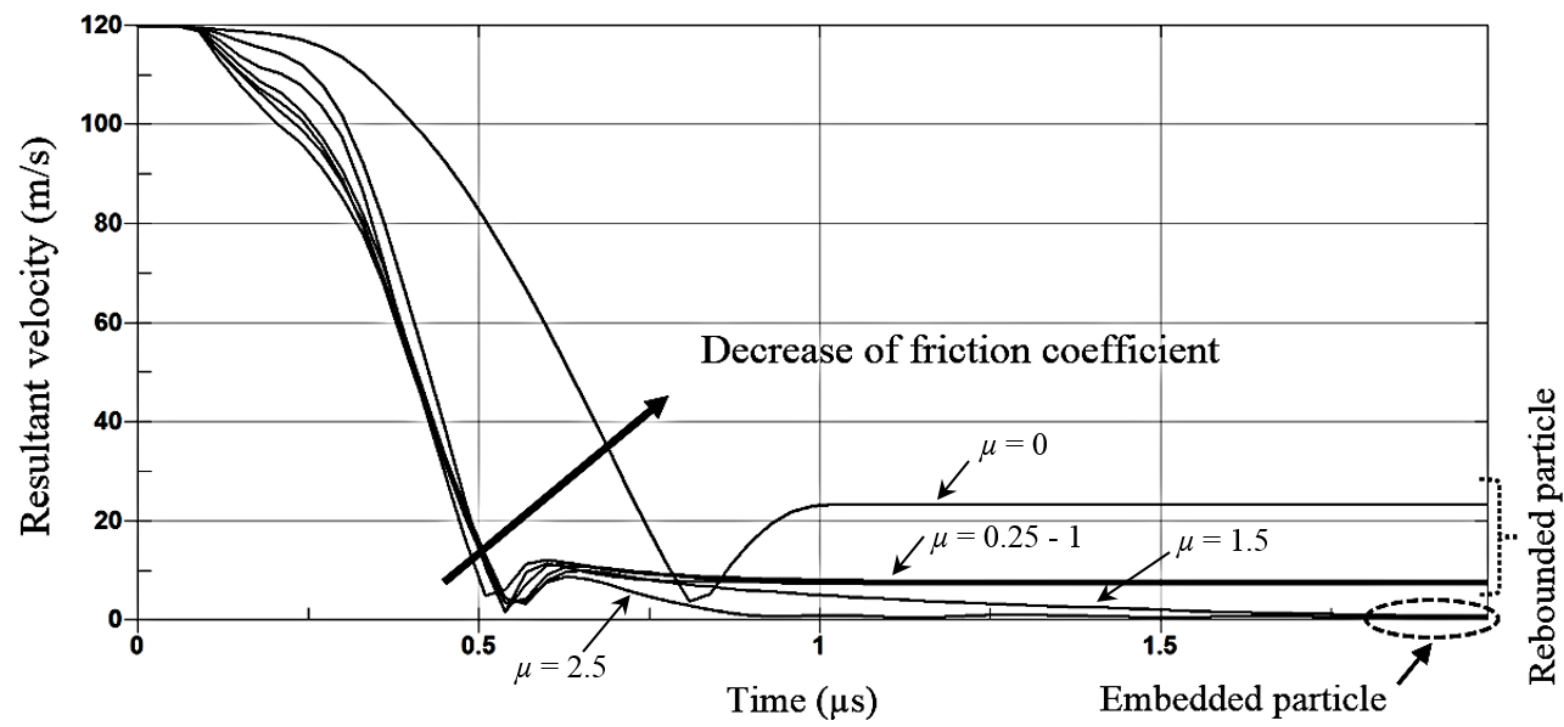

Figure 3.6 Variations of particle center of mass velocity vs impact time for different values of friction coefficient (0-2.5) for a typical grit 90 particle, $\theta=5^{\circ}$, Impact angle $=90^{\circ}, P=300 \mathrm{kPa}$, $V=120 \mathrm{~m} / \mathrm{s}$.

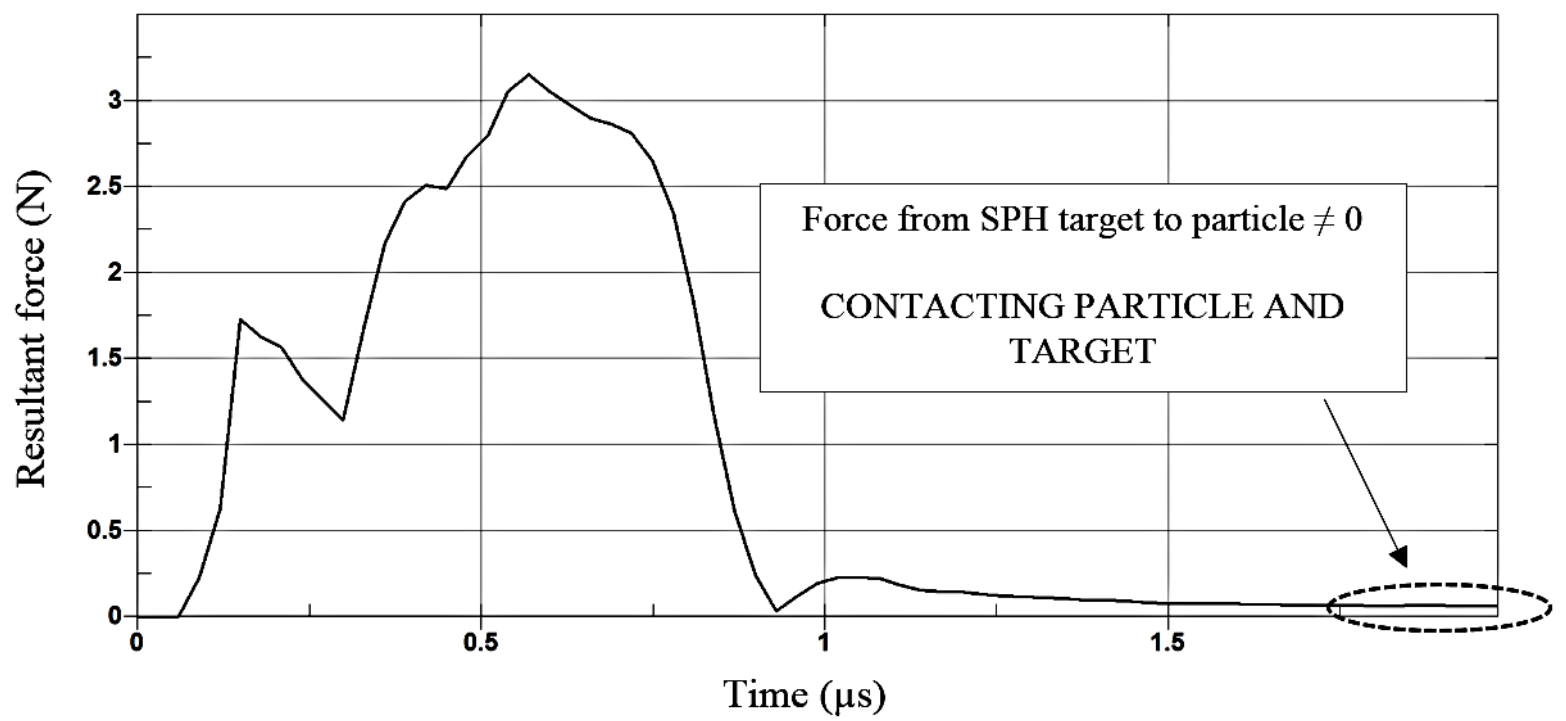

(a) 


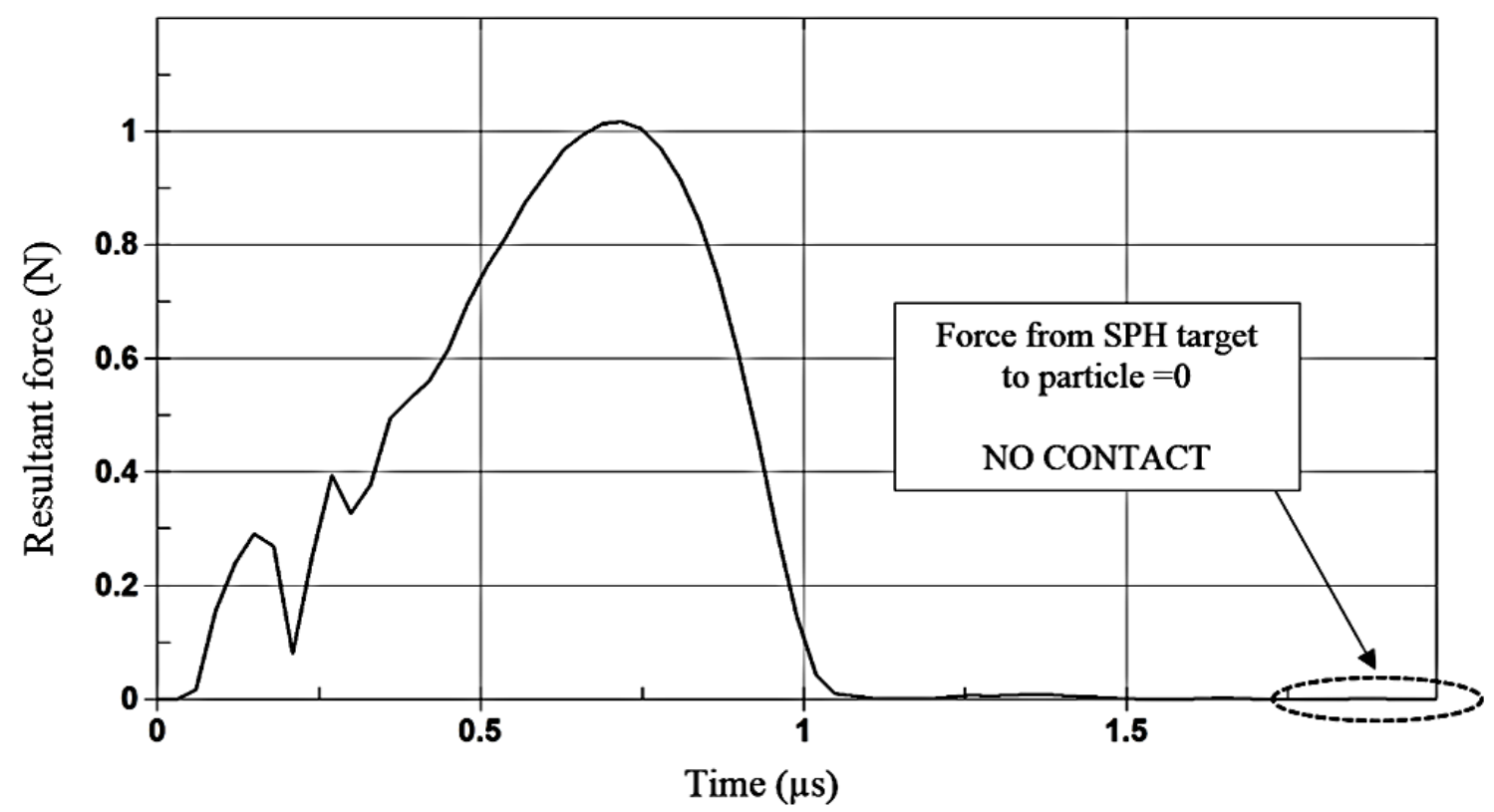

(b)

Figure 3.7 Variations of resultant force from SPH target to (a) an embedded particle, $\mu=1.5$; (b) a rebounding particle, $\mu=0.75$.

\subsubsection{Determination of friction coefficient}

Table 3.3 shows the measured and predicted percentage of embedded grit 90 particles for different values of modeled friction coefficient, for 100 modeled impacts at perpendicular incidence at $300 \mathrm{kPa}(120 \mathrm{~m} / \mathrm{s}$ average velocity). Depending on mass flow rate and exposure time, between 48 and 89 total impact sites (embedded plus impact crater) were found in the experiments which were repeated 3 times.

Table 3.3 Model predicted (100 incident particles) percentage of the embedded particles using different friction coefficients, and corresponding measured value. Experimental conditions: grit $90 \mathrm{SiC}$ abrasives, perpendicular impact, $P=300 \mathrm{kPa}$, Impact angle $=90^{\circ}, \mathrm{V}=120 \mathrm{~m} / \mathrm{s}$.

\begin{tabular}{|c|c|c|c|c|c|}
\hline Experiment & $\begin{array}{c}\text { Numerical } \\
\mu=0.75\end{array}$ & $\begin{array}{c}\text { Numerical } \\
\mu=1\end{array}$ & $\begin{array}{c}\text { Numerical } \\
\mu=1.4\end{array}$ & $\begin{array}{c}\text { Numerical } \\
\mu=1.5\end{array}$ & $\begin{array}{c}\text { Numerical } \\
\mu=2.5\end{array}$ \\
\hline $12.2 \%$ & $5 \%$ & $6 \%$ & $9 \%$ & $11 \%$ & $14 \%$ \\
\hline
\end{tabular}


Table 3.3 indicates that a static friction coefficient of 1.5 results in a reasonable fit between the predicted and measured embedment data. To the knowledge of the author, friction coefficient data between a $\mathrm{SiC}$ grain and an $\mathrm{Al}$ alloy does not exist. Although coefficients of friction do not only depend on material properties, comparisons of the determined coefficient with others reported in the literature can be made, to lend confidence that the determined one is physically reasonable. Given the relatively high dynamic friction coefficients of 0.8-1.4 reported in the literature for other ceramic/metal pairs [122], the present value does not seem unreasonable, and was used in all further models. For example, the friction coefficient between a rough titanium surface and single crystal $\mathrm{SiC}$ at $25^{\circ} \mathrm{C}$ and a sliding velocity of $0.7 \mathrm{~mm} / \mathrm{min}$ in vacuum condition was reported to be between 1 and 1.4 [123-125].

\subsubsection{Prediction of embedding}

Figures 3.8-3.10 show that the models that utilized $\mu=1.5$ were able to quite accurately predict the embedment for combinations of particle size, velocity, and angle of attack, despite the simplifications that were made, which included neglecting particle deformation, assuming a uniform particle thickness and a constant friction coefficient. In most cases, the model slightly over-predicted embedment. Overall, the ratio between predicted to measured embedment was in the range of 0.86-1.06, with an average of 0.98. The effect of various parameters are discussed in the following sections.

\subsubsection{Effect of impact angle}

As expected, both the numerical and experimental results in Figs. 3.8 and 3.9 showed that the percentage of embedded particles at a given velocity and for a given particle size, decreased with decreasing impact angle, in agreement with the findings of Getu et al. [16] for polymers. 


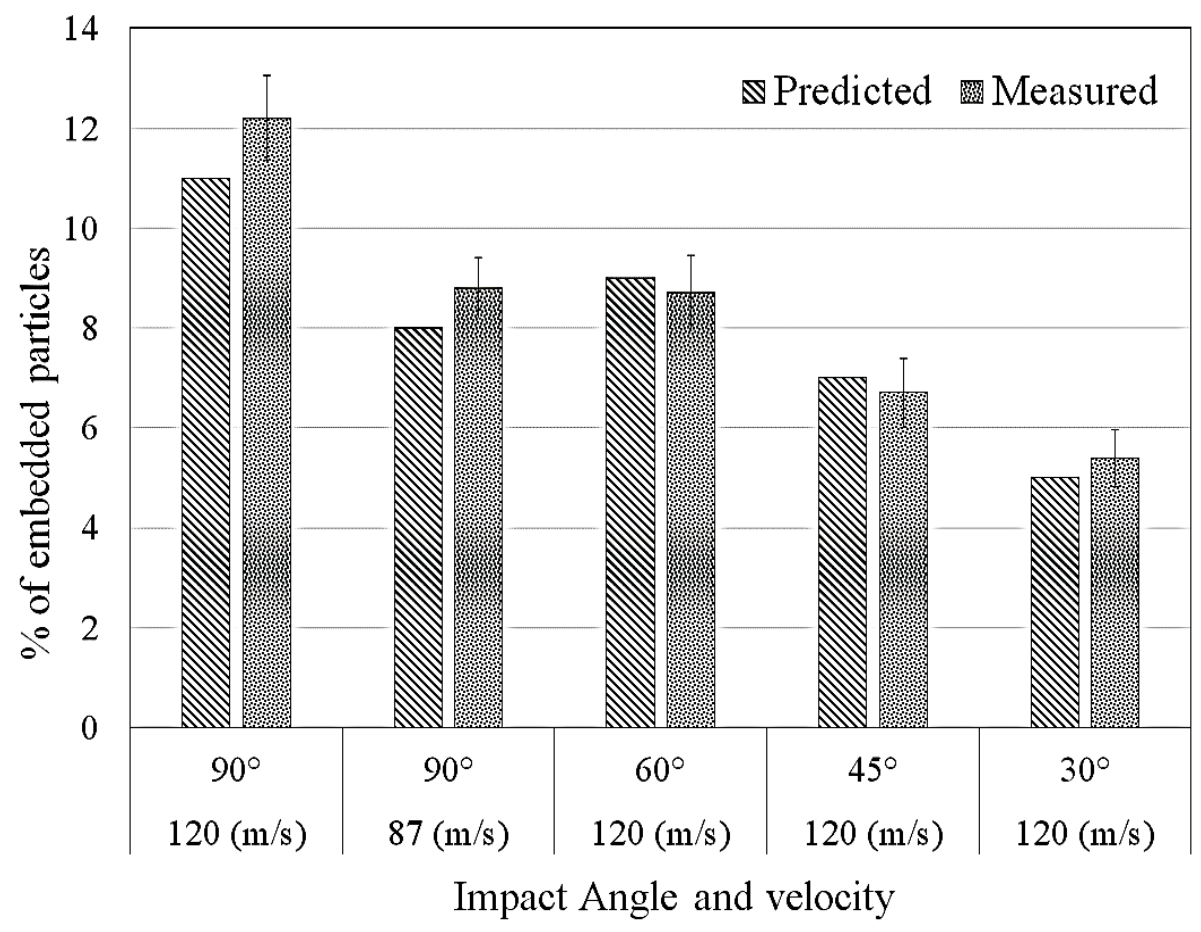

Figure 3.8 Comparison of predicted and measured percentage of particle embedment at various impact angles and two velocities, for grit 90 particles. Scatter bars show the standard deviation of 3 repeated experiments. 100 total impacts modeled.

Analysis of the orientation angle of the modeled particles that embedded in the target demonstrated that in all cases between $68 \%$ to $88 \%$ of them hit the target with an orientation angle of less than $20^{\circ}$ and that particles with an orientation angle greater than $30^{\circ}$ did not embed. This is in general agreement with references [16,99] where it was hypothesized that particles that are oriented with the jet axis are more likely to embed in the target. For example, Getu et al. [16] predicted that most of particles that embed into a polymer under $90^{\circ}$ impact angle, hit the target with an orientation angle of less than $20^{\circ}$. Moreover, they reported no particle embedment at orientation angles of larger than $25^{\circ}$.

\subsubsection{Effect of particle size and velocity}

Previous measurements under identical conditions to the present, showed that distribution of orientation angles and roundness (Table 3.1) for grit 60 and 90 particles at the same standoff distance were approximately the same [99]. This made it possible to directly compare the effect 
of particle size and velocity on embedment, by conducting further blast experiments and numerical simulations using grit $60 \mathrm{SiC}$ abrasives (Table 3.1).

In order to compare the embedment of grit 60 and 90 abrasives, numerical simulations and experimental measurements were conducted using same velocity $(87 \mathrm{~m} / \mathrm{s})$, standoff $(20 \mathrm{~mm})$ at various impact angles (Table 3.1). Comparison of Figs. 3.8 and 3.9 reveals that the percentage embedded for grit 90 particles was lower than that of grit 60 . For example, $16.2 \%$ of the grit 60 particles embedded, approximately 1.79 times more than for grit 90 particles under the same conditions. Thus, at the same velocity, larger particles were more likely to embed in the target.

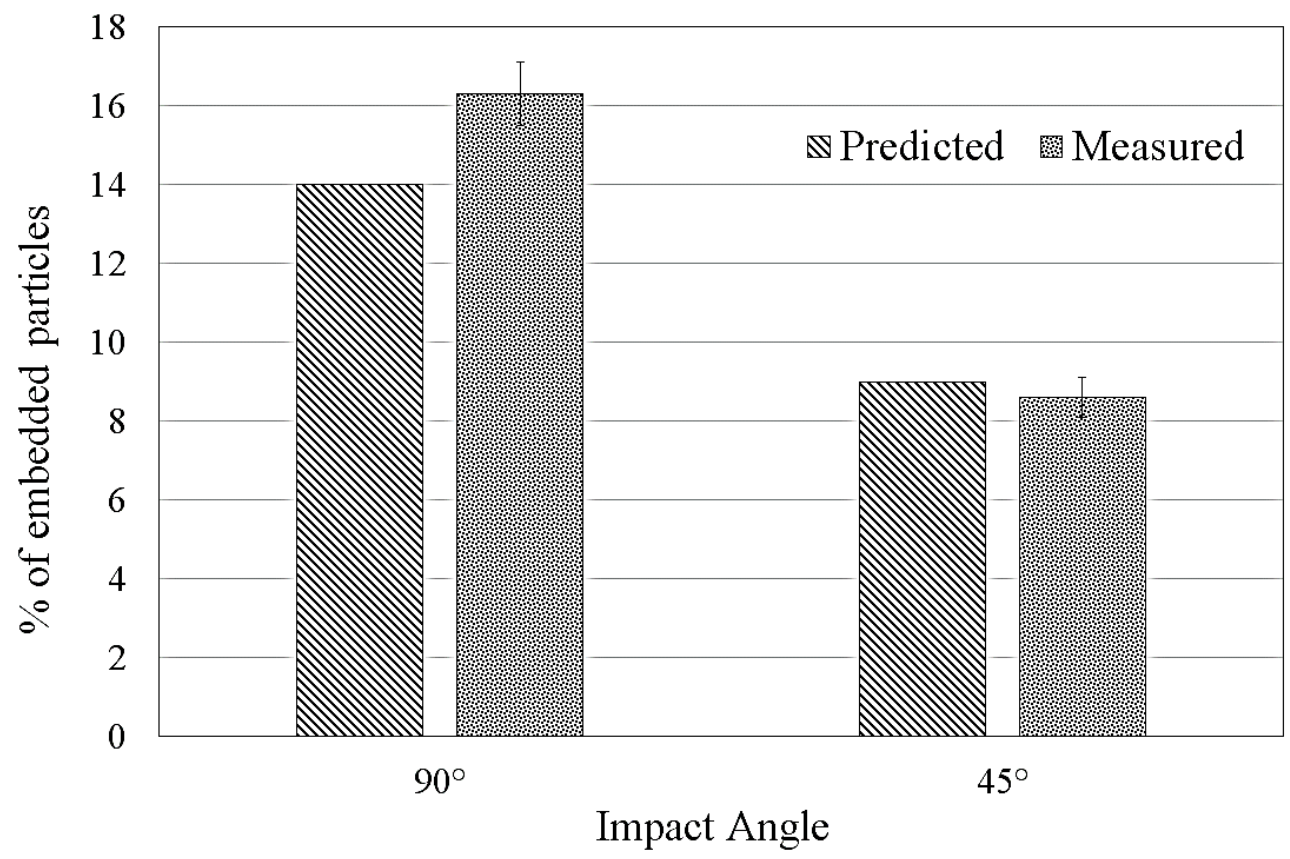

Figure 3.9 Comparison of predicted and measured percentage of particle embedment at $V=87$ $\mathrm{m} / \mathrm{s}$ (grit 60 , standoff $=20 \mathrm{~mm}, P=300 \mathrm{kPa}$ ). Scatter bars show the standard deviation of 3 repeated experiments. 100 total impacts modeled.

As shown in Fig. 3.8, an increase in velocity of grit 90 particles from $87 \mathrm{~m} / \mathrm{s}$ to $120 \mathrm{~m} / \mathrm{s}$ while other parameters were the same, resulted in a higher embedment percentage. The influence of a greater range in particle velocities on embedment was investigated by modelling the impact of grit 60 particles at a larger $40 \mathrm{~mm}$ standoff distance using air pressures of $100 \mathrm{kPa}$ and $500 \mathrm{kPa}$, corresponding to 39 and $115 \mathrm{~m} / \mathrm{s}$ average particle velocity, and comparing to experiments. As 
shown in Fig. 3.10, this increase in particle velocity resulted in an approximately 5 times higher embedment.

Another set of numerical simulations for the same package of models at $87 \mathrm{~m} / \mathrm{s}$ showed $15 \%$ embedded particles, forming an approximately linear correlation that predicts that there should be no embedment for this combination of process parameters at incident velocities below a certain threshold. Indeed, numerical simulations showed no embedded particles below $16 \mathrm{~m} / \mathrm{s}$. Equipment limitations unfortunately made it impossible to test this embedment threshold.

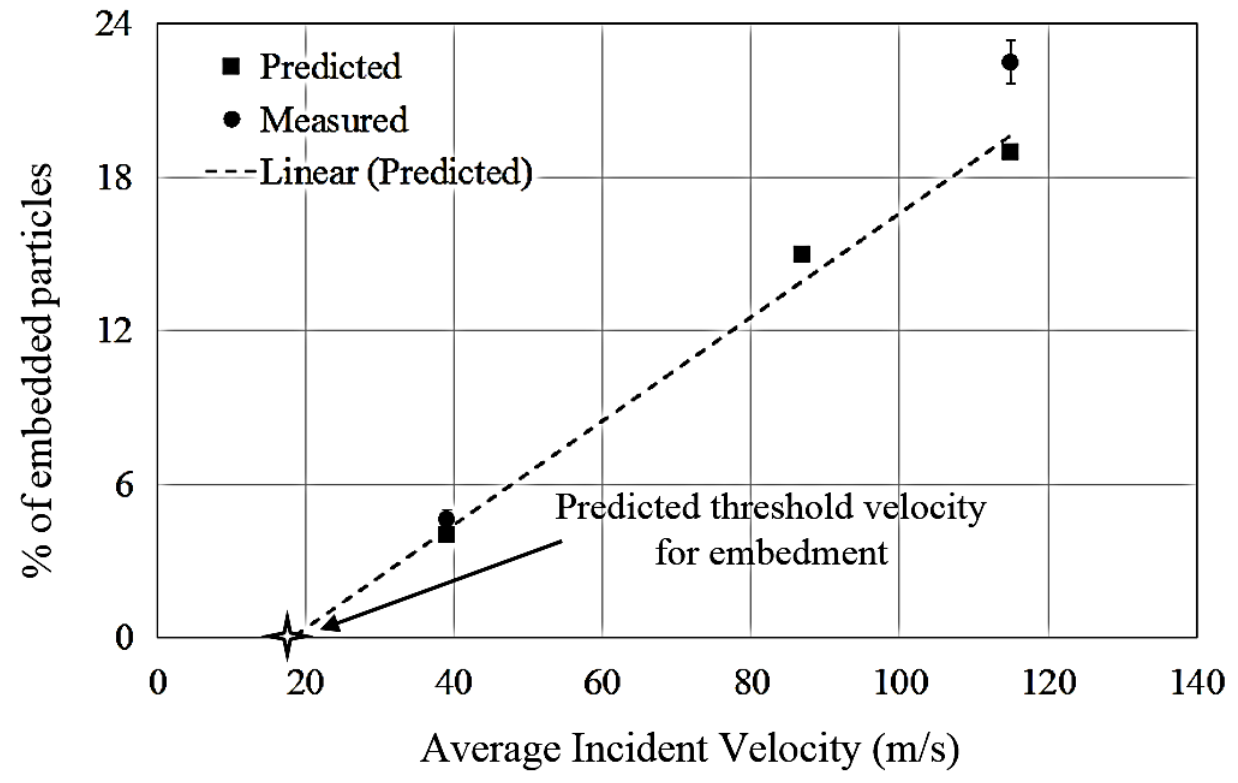

Figure 3.10 Comparison of percentage of embedded grit 60 particles at various velocities at 40 $\mathrm{mm}$ standoff and $90^{\circ}$ impact angle.

Comparison between the percentage of embedded particles at $20 \mathrm{~mm}$ and $40 \mathrm{~mm}$ standoffs (impact velocity $=115 \mathrm{~m} / \mathrm{s}$ and $120 \mathrm{~m} / \mathrm{s}$, respectively) of grit 60 particles shows that the percentage of embedded particles was 1.05 times higher. Numerical analysis revealed that the relatively small $\sim 4 \%$ difference in incident velocity had no significant influence on embedment. However, the percentage of particles oriented (orientation angle less than $30^{\circ}$ ) was approximately 1.1 times higher at $40 \mathrm{~mm}$ than $20 \mathrm{~mm}$ [99]. This implies that the increase in the percentage of embedded particles was due to the increase in the amount of oriented particles, rather than the small increase in velocity. 
For a constant pressure of $300 \mathrm{kPa}$, at a $20 \mathrm{~mm}$ standoff, although the average velocity of the smaller grit 90 particles at the same pressure was 1.4 times greater than grit 60 particles, the percentage of the larger embedded grit 60 particles nevertheless increased by 1.34 and 1.28 times at $90^{\circ}$ and $45^{\circ}$, respectively (Fig. 3.8 and 3.9). This indicates that particle size affects embedment to a greater extent than velocity.

In summary, embedment increased with increasing particle size at the same velocity, in agreement with the experiments of, e.g. Akbarzadeh et al. [50], but contrary to those of Getu et al. [16]. Embedment increased with velocity for the same particle size, in agreement with the experimental results of, e.g., Day et al. [98]. As will also be discussed in Section 3.4.4, these trends can be linked to the kinematics of the particles as they strike the surface, and whether contiguous contact is maintained. For example, Fig. 3.11 shows the impact of two identically shaped particles, one of size grit 60 , and the other size grit 90 , at the same velocity, with the grit 60 embedding and the grit 90 rebounding. It is evident that the smaller particle rotated more than the larger one, causing it to lose contact with the target and rebound. Although the smaller particle had a lower rotational moment because of its associated lower moment arm and impact force, the lower mass and dimensions generated a much lower moment of inertia. Assuming a rhomboid shape, the moment of inertia of the particle is proportional to $\frac{1}{6} \rho h^{4}$ where $\rho$ and $h$ are the density and side length of the particle. Therefore the angular acceleration is approximately proportional to $F / h^{3}$, where $F$ is the resultant of the contact force. This implies that, even if the contact force is much lower in the case of a small particle, the lower $h$ will dominate, leading to a higher propensity to rotate and lose contact (i.e. not embed). Moreover, the deeper penetration of the larger particle (Fig. 3.12) meant that there was more target material in contact to resist the rotation once it started.

Similarly, when the kinematics of two identical particles but at different velocities were compared, with the higher velocity one embedding and the lower not, less rotation occurred for the higher velocity particle. This was because when the higher velocity particle started to rotate, its deeper penetration meant that there was more material to resist rotation on the opposite side of the particle. 

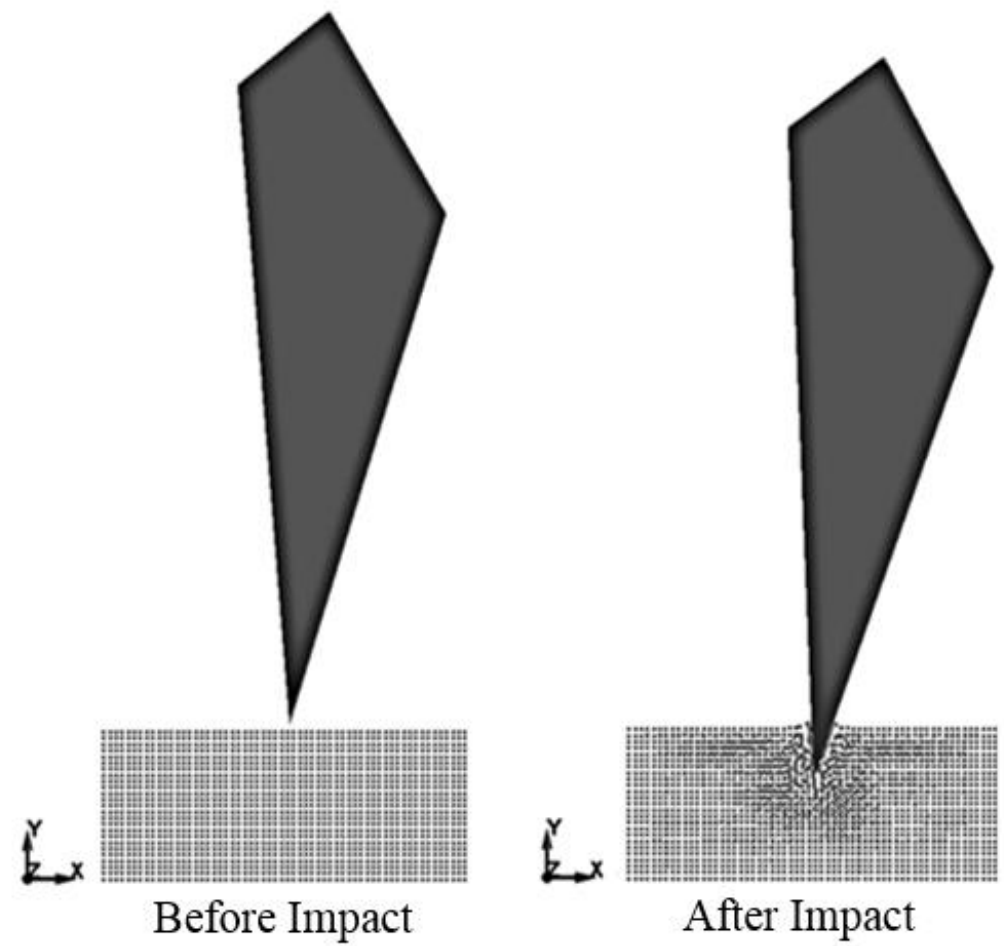

(a)

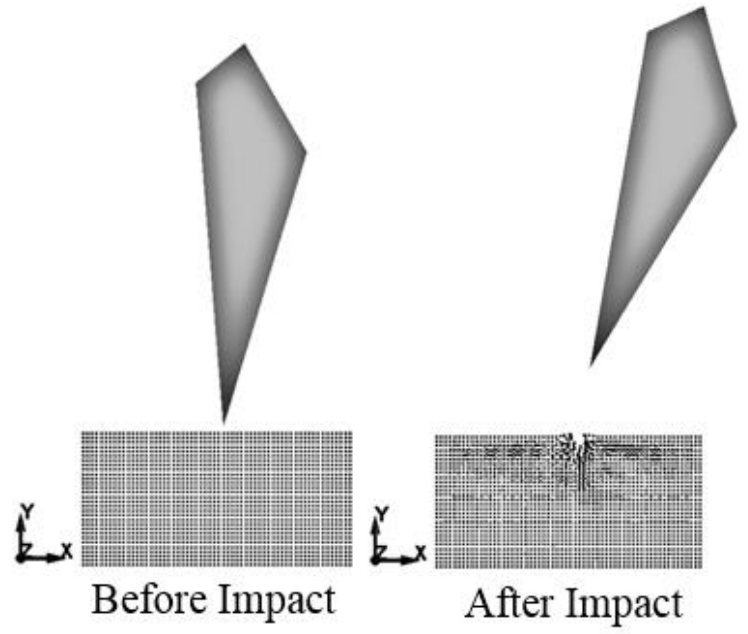

(b)

Figure 3.11 Impact and rotation of identically-shaped particles at $87 \mathrm{~m} / \mathrm{s}$ velocity and $90^{\circ}$ impact angle for (a) grit 60 (embedment), and (b) grit 90 (rebounded). 


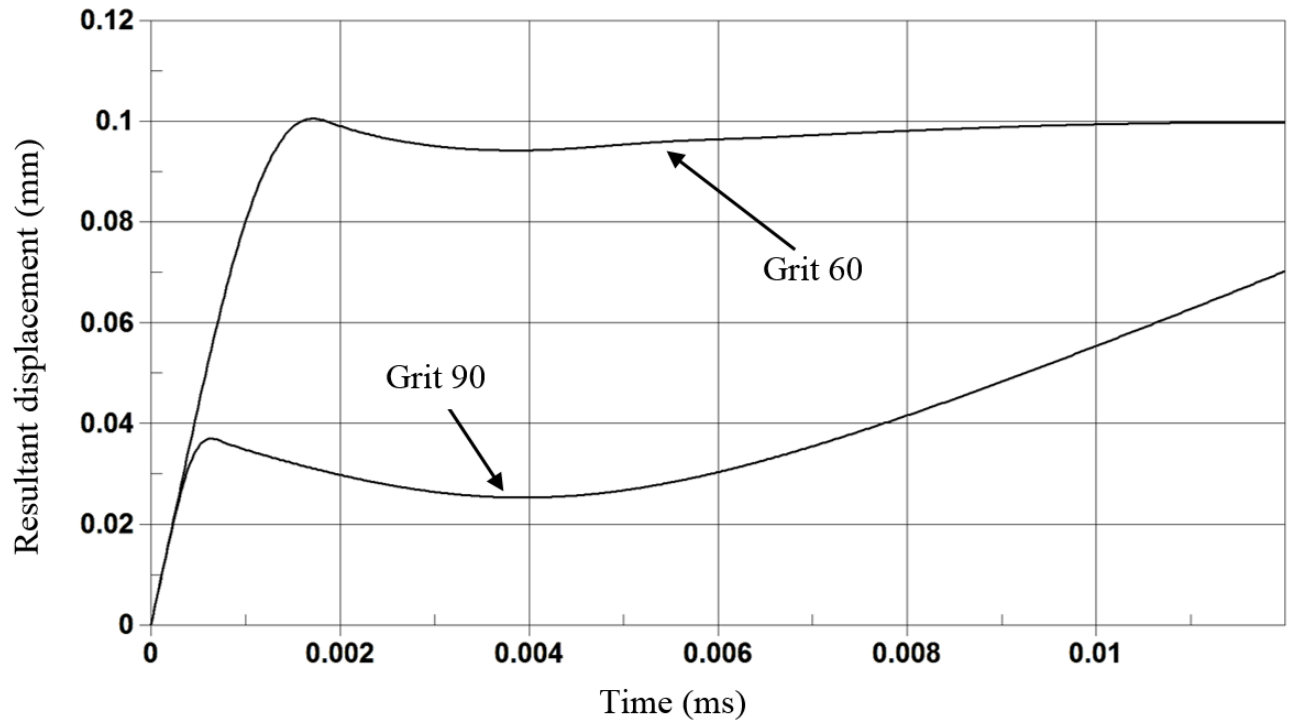

Figure 3.12 Resultant displacement of particle center of mass versus time for the two particles of Fig. 3.11 .

\subsubsection{Embedding mechanisms}

Using a simplified analysis which balanced the elastic rebound and frictional forces, Getu et al. [16] concluded that, for an elastic, perfectly plastic material, a necessary condition for the embedment of a rhomboid particle of angularity $A$ (Fig.3.13), was that the static friction coefficient between the particle and target be at least,

$$
\mu_{c}=\frac{1}{\tan (A)}
$$




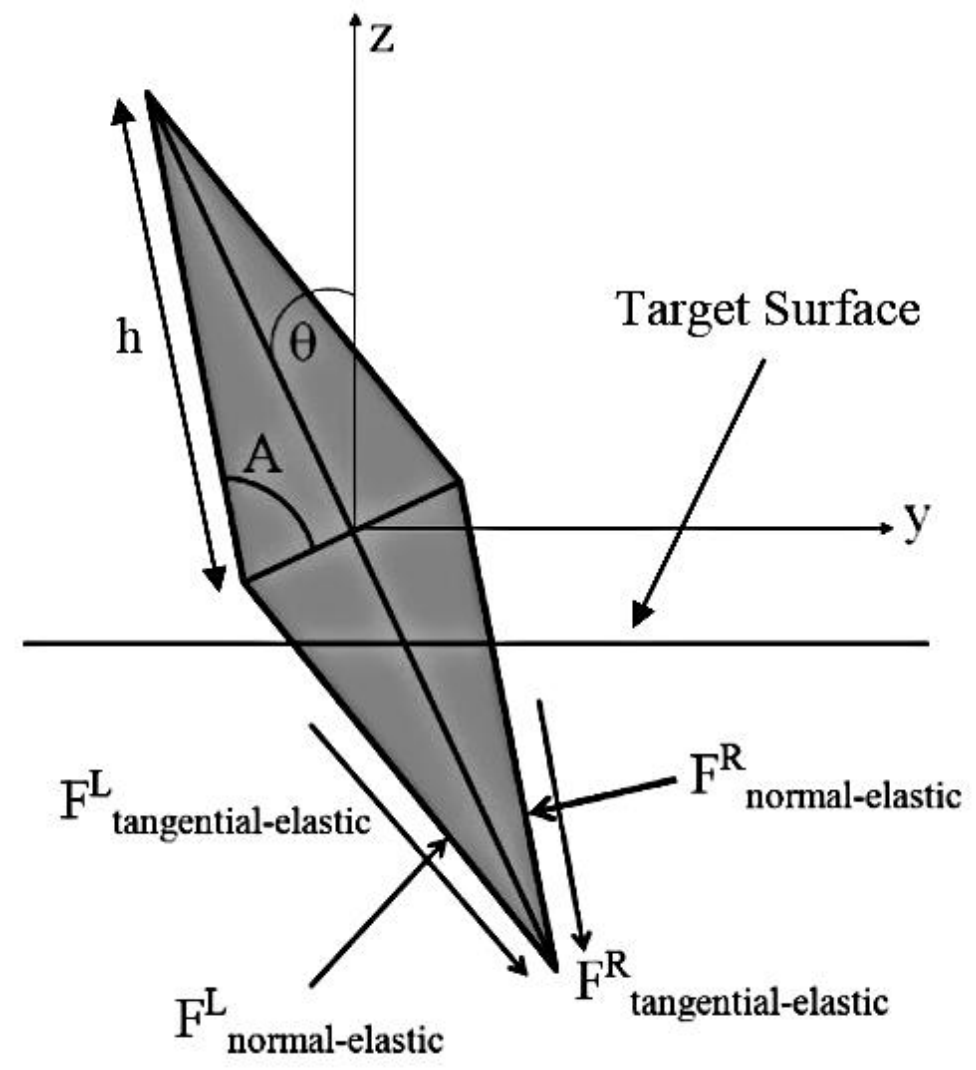

Figure 3.13 Forces acting on an angular particle in contact along both faces [16]. $F_{\text {normal-elastic }}{ }^{L}$ and $F_{\text {normal-elastic }}{ }^{R}$ are the elastic normal forces and $F_{\text {tangential-elastic }}{ }^{L}$ and $F_{\text {tangential-elastic }}{ }^{R}$ are the tangential forces acting on a particle upon rebound. $A$ is the angularity and $\theta$ orientation of particles.

Getu et al. [16] thus required that the system provide a critical amount of friction, depending on the particle angularity $(A)$, in order for his idealized rhomboid particles to embed. Although the study of ref. [16] assumed idealized particles impacting an idealized (rigid-plastic) material, since it is the only other existing model of embedment, it was of interest to draw some comparisons. Assuming the present particles to be ideal rhomboids, and noting that both grit 60 and 90 had similar shapes (roundness, Table 3.1), their angularity was calculated based on their measured length and width distributions. Figure 3.14 shows the critical friction coefficient (eq. 3.14) required for embedment of the present particles, according to Getu et al.'s criterion. 


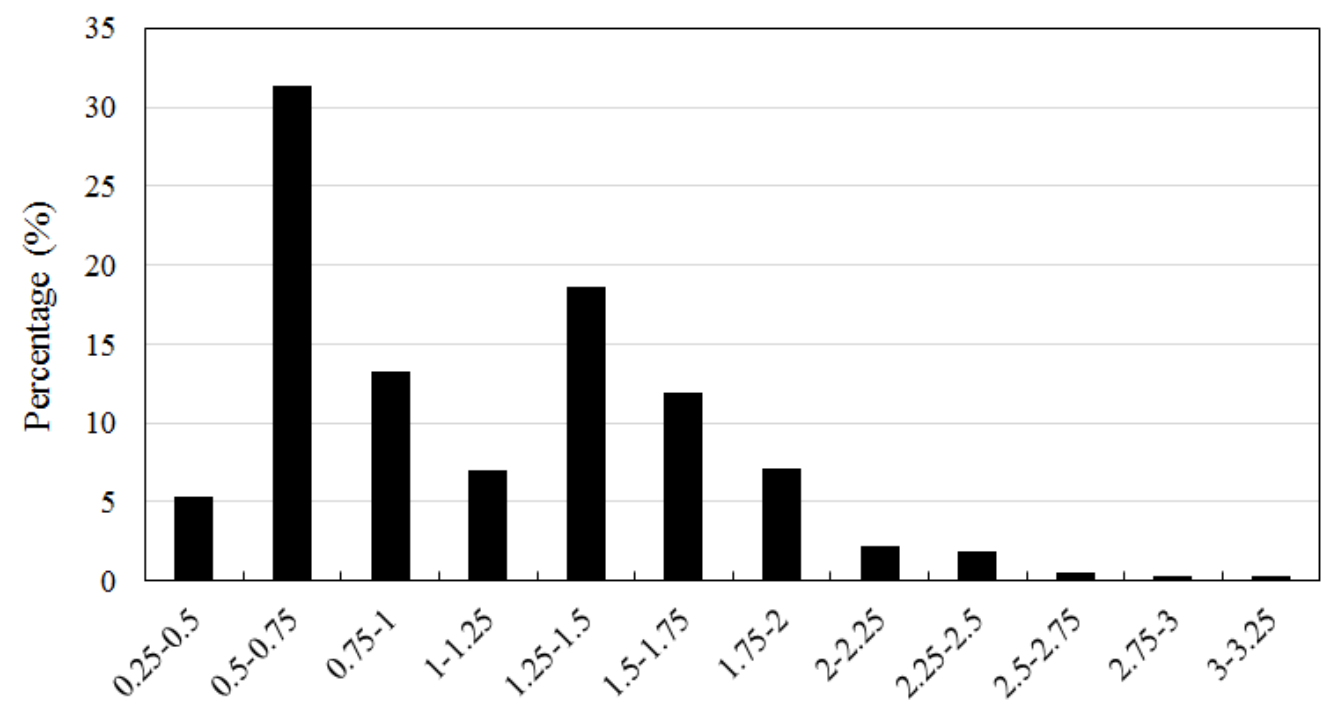

Critical value of static friction coefficient

Figure 3.14 Distribution of critical value of friction coefficient as predicted by Getu et al. [16] for equivalent ideal rhomboid particles. All incident particles considered.

It can be concluded that more than $75 \%$ of the incident particles in the present system had a critical friction coefficient less than 1.5, i.e. they meet Getu et al.'s criterion [16].

Figures 3.15a and 3.15b show the distribution of critical friction coefficient for those incident particles that actually were predicted to embed in the numerical simulations at $120 \mathrm{~m} / \mathrm{s}$ for both particle sizes. The distribution of angularities of embedded particles, and therefore the critical friction coefficients, were similar, but the average critical friction coefficient was slightly larger (0.65, compared to 0.59) for the grit 60 particles, i.e. the larger particles allowed for less angular particles to embed than the smaller ones $\left(60^{\circ}\right.$ compared to $\left.57^{\circ}\right)$. A similar analysis (not shown) of critical angularity/friction coefficient showed that slightly less angular particles embedded into the target at higher incident velocity $\left(56.5^{\circ}\right.$ at $115 \mathrm{~m} / \mathrm{s}$, compared to $61.1^{\circ}$ at $\left.39 \mathrm{~m} / \mathrm{s}\right)$. In all cases, the critical friction coefficient was less than 1.5, and therefore all the embedded particles met the criterion in Getu et al.'s work [16]. However, Getu et al. also required that the particle maintain contiguous contact during impact in order to embed. Analysis of the numerically predicted contact forces indicated that indeed full contact was maintained on both sides of those particles that embedded, while those that did not embed lost contiguous contact during the impact, rotated, and rebounded. These observations reinforce the arguments of Section 3.4.3.2 regarding the 
differences in rebound kinematics and propensity to maintain contact occurring when smaller versus larger and higher velocity vs lower velocity particles impact the target.

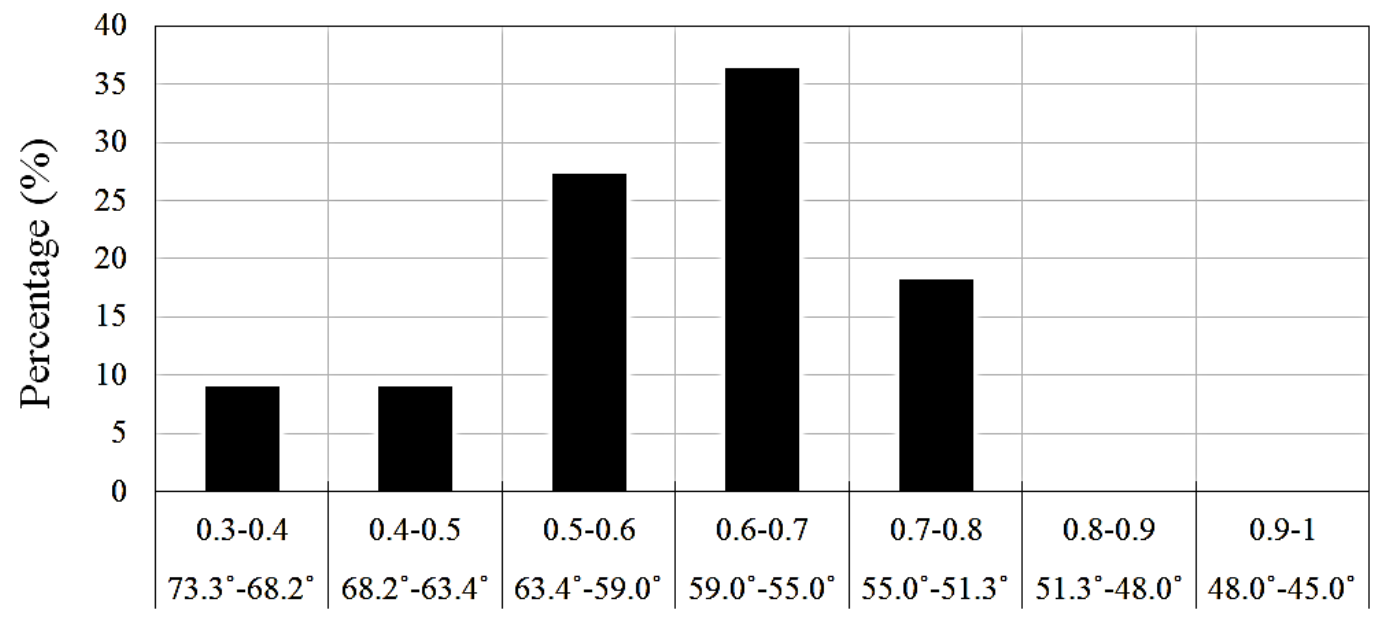

Critical friction coefficient and angularity

(a)

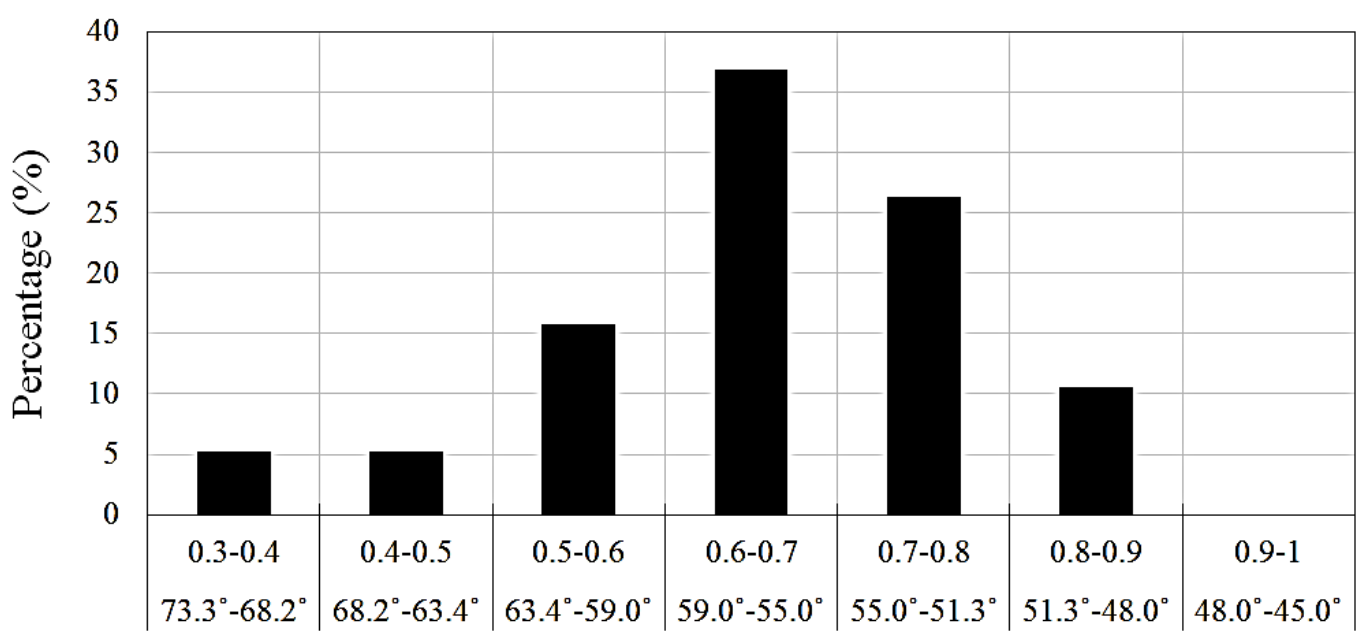

Critical friction coefficient and angularity

(b)

Figure 3.15 Distribution of critical value of friction coefficient for modeled embedded particles at $120 \mathrm{~m} / \mathrm{s}, 90^{\circ}$ impact angle (a) grit 90, (b) grit 60.

\subsection{Summary}

Coupled FEM and SPH modeling was utilized for the first time to simulate the impact and embedment of different single abrasive particles drawn from distributions of various sizes at 
various process conditions on a ductile metal that strain and strain rate hardened. The friction coefficient was found to strongly affect the likelihood of embedment, and was determined as 1.5 by comparing the predicted and measured embedment percentage from the numerical model for one particle size, standoff, and velocity at perpendicular incidence. Use of the determined coefficient in other models utilizing different particle sizes, impact angles, standoffs, and velocities yielded predictions that were at most 1.16 times higher than measured ones, thus validating the model.

Consistent with Ref. [16,99], it was shown the likelihood of embedment strongly depended on the particle orientation. Particles that hit the target in orientations that were not aligned with the jet were found to not embed even when using large friction coefficients. This demonstrated the importance of using realistic particle orientation angles in numerical modeling of erosion. All other parameters being equal, the percentage of embedded particles was shown to increase with increasing standoff distance due to the increase in the percentage of particles oriented with the jet. Numerical modeling confirmed that particles striking the target with an orientation angle larger than $30^{\circ}$ generally did not embed, and that the vast majority of embedded particles had orientation angles below $20^{\circ}$, that the likelihood of particle embedment increased for highly oriented particles. The models and experiments showed that, for the same shape and impact velocity, larger particles were more likely to embed, in agreement with the findings reported by Akbarzadeh et al. [50]. It was also shown that for a given particle size, increases in particle velocity enhanced the likelihood of particle embedment, consistent with the reports in Day et al. [98]. The reasons for these trends were the greater tendency of smaller particles to rotate and the stronger resistance against particle rotation under higher impact velocity. Finally, the study indicated that there was a minimum critical velocity required for embedment to occur.

The model presented in this chapter will be modified in Chapter 4 to allow prediction of particle fracture due to impact, by utilizing a Johnson-Holmquist material model for the particle, and by applying a new technique for generation of CAD representations of the particles. 


\section{Numerical Modeling and Experimental Analysis of Particle Fracture During Solid Particle Erosion}

This chapter is based on the following paper:

V. Hadavi, C. E. Moreno, M. Papini, Numerical and Experimental Analysis of Particle Fracture During Solid Particle Erosion, Part I: Modeling and Experimental Verification, Wear (Article in Press)

\subsection{Introduction}

\subsubsection{Abrasive particle fracture due to impact}

Material wear due to the impact of a high speed jet of abrasive particles, i.e. solid particle erosion, occurs in many industrial applications including steam and wind turbomachinery [126], pipeline wear [127,128], to clean and strip surfaces [129], and to machine surfaces [130]. Despite the fact that the energy consumed in particle fracture can affect the erosion rate and mechanisms $[48,50,131]$, this aspect of solid particle erosion has received relatively little attention in the literature. Most investigations have focused on the target material removal mechanisms associated with the impact of non-deforming erodent particles, because the abrasives usually are relatively hard [132-134]. However, hard particles are generally relatively friable, and thus may fracture upon impact. For example, as early as 1970, Tilly and Sage [48] reported that impacts between abrasive particles at approximately $91 \mathrm{~m} / \mathrm{s}$ and metal, nylon and fiberglass target not only resulted in target material extrusion and plastic deformation, but also abrasive fracture.

Fragmentation of particles can influence the material removal mechanism in a number of ways. As first suggested by Tilly and Sage [48], radial translation of particle fragments may leave impact craters that can result in secondary erosion [48], and may also result in a larger eroded footprint than expected, with the interaction between the impacting particles and the fragments

resulting in further increases. Akbarzadeh et al. [50] suggested that such interactions may serve to reduce the effective kinetic energy content of the jet, thus reducing the erosion rate. In some cases, only $20-30 \%$ of the initial energy of a typical particle is transferred to the fragments [48]. Slikkerveer and in't Veld [135] utilized a one-dimensional Laser Doppler system to determine the rebound characteristics of a jet of $23 \mu \mathrm{m}$ alumina at $200 \mathrm{~m} / \mathrm{s}$, and reported that the rebound velocity 
after impact on a glass surface was between $0.2-0.5$ of the incident velocity. Winter and Hutchings [136] suggested that, when cutting or ploughing a crater, if a small angle between the substrate and cutting edge of the abrasive is formed, the fracture of particle occurs in a plane perpendicular the leading/cutting edge, resulting in two separate fragments, each of which can separately erode the surface on two planes. In this scenario, the crater lips generated by the initial impact may be removed by the secondary impact due to the fragments. Winter and Hutchings' study [136] illustrated that abrasive fracture was possibly more influential on erosion than Tilly and Sage [48] proposed.

Several studies have focused on determining the minimum load required for particle fracture. Nahvi et al. [137] measured the crushing load of bottom-ash abrasives and silica particles by crushing them between steel surfaces coupled to a load cell. They found that sudden fracture of silica abrasives occurred at a critical load. Nouraei et al. [138] utilized an apparatus to crush single abrasive particles, and found no significant difference between the crushing load of dry and wet alumina particles. Murugesh and Scattergood [139] studied the indentation of $\mathrm{Al}_{2} \mathrm{O}_{3}$ and $\mathrm{SiC}$ abrasives on alumina targets of different hardness and reported that fragmentation affected the erosion and that the impact load influenced the likelihood of particle fracture. They found that the threshold load was directly proportional to the hardness of the particles. They also suggested that the average particle sharpness may also decrease due to fracture, thus affecting the material removal mechanism and rate.

In order to study particle fracture independently of particle interaction effects, some investigators have performed single impact studies using a gas gun [48, 49]. For example, Salman et al. [49] studied the fracture of single spherical $\mathrm{Al}_{2} \mathrm{O}_{3}$ particles of $5.15 \mathrm{~mm}$ diameter due to impact with a flat target at incident velocities up to $35 \mathrm{~m} / \mathrm{s}$. They detected two different modes of fragmentation; i.e., normal and oblique fragmentation occurring at perpendicular and shallow impact angles, respectively. The fractured surface for normal fragmentation was smooth and flat and fragments were rather symmetrical and of equal size. On the other hand, the fractured surfaces for oblique fragmentation were rough and the fragments were dissimilar [49].

Target material properties can also affect abrasive fracture. Salman et al. [49] utilized plexiglass, glass, steel, and aluminum targets and reported that, for a given incident velocity larger than a threshold value, the number of fractured particles was highest for steel, and lowest for plexiglass targets. They thus concluded that particle fracture is less likely to occur on softer targets 
because a larger portion of the particle kinetic energy is transferred to a soft target, making less energy available to cause the fracture [49]. They also found that increasing target thickness, up to a threshold value, tended to increase the number of fractured particles due to the greater tendency for thin targets to absorb kinetic energy in bending [49].

There have been very few attempts to develop analytical models of particle fracture due to impact. Murugesh and Scattergood [56] presented an analytical model to predict the fracture of abrasives impacting an alumina target, based on the hypothesis of Lawn and Evans [57] with regards to the presence of a critical penetration depth to cause fracture of a brittle material. In their model, Murugesh and Scattergood assumed the existence of a critical penetration depth of the target into the particle required for fracture of the particle [56]. The likelihood of particle fracture was found to depend on the ratio of the fracture toughnesses, hardnesses and Young's moduli of the abrasive and target, as well as the intrinsic flaw population of the two materials. They evaluated their model by measurement of the erosion rate using alumina and $\mathrm{SiC}$ abrasives, and reported that the erosion rate decreased with an increase in the number of fractured particles [56].

In summary, despite some intriguing experimental data, there appears to be only one particle fracture model. It is based on the assumption of a crack propagation due to impact with a brittle target, making it unsuitable for application in cases where the target is ductile. Moreover, the model cannot be used to predict the size and shape of particle fragments as a function of particle size, shape and other process parameters. Such predictions can only be obtained using numerical simulations that consider actual particle geometries.

\subsubsection{Numerical modeling of fracture due to impact}

Since the present work will utilize numerical models of particle fracture due to impact, a brief review of numerical techniques in impact fracture mechanics is appropriate. Cracks are due to intensive strain localization which ultimately results in fracture [58], and two approaches have been suggested to numerically simulate this localization, the re-meshing method, and the local approach. In the re-meshing method, the discontinuity at the highly strained zone at the crack tip is re-meshed [140,141] at each crack extension. For example, Rashid [140] applied the re-meshing technique to a finite element simulation of cracking for a fully brittle material. In the local approach, the cracking evolution is described through micromechanical models. For example, Xie 
and Waas [142], and Qian and Xie [143], developed a detaching algorithm which can simulate the separation in neighboring elements during cracking.

The discretization of the domains used in numerical modeling of fracture can be generally categorized into two main approaches, i.e., the grid based and mesh free [144]. A critical issue in the modeling of cracks using grid based finite element methods is how to generate the cracked surface explicitly [145]. The deficiencies of grid-based methods in the simulation of impact problems mainly involve the existence of instabilities due to large deformation and element distortion. Problems with element distortion can be avoided using mesh free techniques $[32,111,112]$ in which there is no connectivity among the nodes. Smoothed particle hydrodynamics (SPH) is a powerful mesh free method developed by Lucy [146] and Gingold and Monaghan [147] which has been broadly applied in simulation of large deformation problems and fracture of solid materials. Libersky and Petschek [148] and Swegle et al. [60] applied SPH to solid mechanics for the first time. The SPH method is capable of simulating micro voids; but the continuity of the material and the relationship between the nodes described by the smoothing function depends on the interpolation length [65], thus preventing accurate crack modeling. To address this, Randles and Libersky [149] proposed that a damage model be applied while decreasing the interpolation length. They suggested that two rows of SPH particles should fail in order to optimize the predicted crack opening width. They also proposed that for maximum accuracy the meshed zone should be refined by splitting the particles in the vicinity of the crack tip.

Belytschko and his colleagues [66,68,150] have made significant contributions in the development of novel numerical techniques for modeling dynamic fracture in solids by introducing the element free Galerkin (EFG) method. EFG applies a moving least squares formulation to generate the numerical discretization. Applying visibility and transformation techniques, EFG can model the crack formation and the boundary conditions in the cracked material $[68,150]$, and thus give insight regarding the stresses along and at the tip of the crack [70]. Belytschko and his coworkers verified the applicability of EFG to model dynamic fracture by comparison with measurements taken during an impact in the Taylor experiment [66]. The EFG method has since been used in a variety of applications and shown to perform well for the simulation of cracking and fracture in brittle materials without the unfavorable re-meshing required in adaptive FE 
methods. For example, Guo et al. [70] and Morka [69] applied EFG to simulate the fracture of different ceramic parts and found the results in reasonable agreement with experiments.

The use of an appropriate material constitutive model is an essential part of fracture simulation. The Johnson-Holmquist $(\mathrm{JH})$ model $[71,72]$ has been most widely used for ceramics and other brittle materials, and especially in numerical simulation of problems involving large deformation. Two classes of $\mathrm{JH}$ constitutive models have been developed: $\mathrm{JH}-1$ describes the behaviour and strength of ceramics based on a linear approximation method; while JH-2 [73,77] applies a smoothing method and also considers the decrease in strength due to a damage criteria. $\mathrm{JH}$ models have been used in many numerical simulations of ceramic fracture, including studies by Quan et al. [74], Guo et al. [75], Kaufmann et al [77] and Reidel et al. [65]. More relevant to the present work, Wang and Yang [76] used the JH-2 model to model the solid particle erosion of a $\mathrm{SiC}$ target, and reported a reasonable accuracy in comparison with experimental data from the literature.

The present work will present a numerical and experimental analysis of particle fracture occurring in a typical abrasive jet machining application. The numerical models were implemented in LS-Dyna utilizing the JH-2 material model, and EFG formulations were used to simulate the fracture of abrasives. The models were verified by comparison with measurements made using double-pulsed laser shadowgraphy, which made it possible to capture the fracture of the particles

at the instant of impingement. These models and further experiments will be used in Chapter 5 to determine the influence of process parameters such as particle size, incident velocity, impact angle, etc. on the extent of fracture, fragment shape and size, and rebound kinematics.

\subsection{Experiments}

\subsubsection{Erosion experiments}

A commercial micro- blaster (Accuflo, Comco Inc., Burbank, CA, USA) was used to blast grit 60 Silicon Carbide (SiC) abrasive particles with an average circular diameter of $363 \mu \mathrm{m}$ and the geometry described in Chapter 2 at a working pressure of $400 \mathrm{kPa}$. $5 \mathrm{~mm}$ thick Al6061-T6 $(90 \mathrm{BHN})$ targets were placed at a $20 \mathrm{~mm}$ standoff distance from the exit of a $1.5 \mathrm{~mm}$ inner diameter, and $40 \mathrm{~mm}$ long nozzle, at an impact angle of $60^{\circ}$ as shown in Fig. 4.1. The impact angle was chosen as $60^{\circ}$ to ensure that rebounding and incoming particles could be distinguished in the 
shadowgraphy measurements (Section 4.2.2). For the measured mass flow rate of $\sim 3.4 \mathrm{~g} / \mathrm{min}$, the analysis of refs. $[152,153]$ indicated that there was no interaction between the impacting particles.

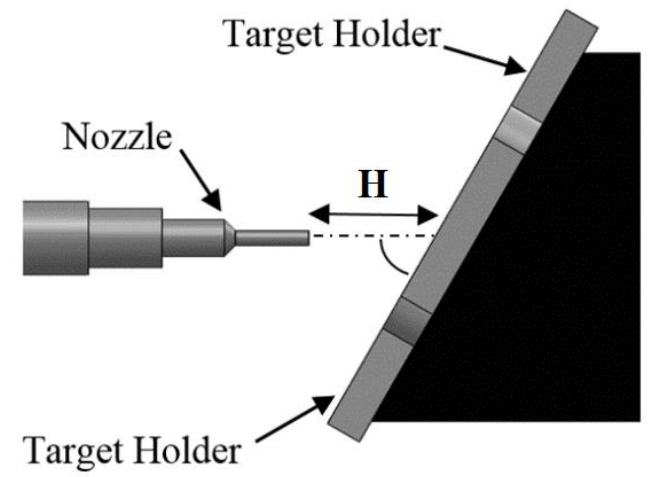

Figure 4.1 AJM setup, using impact angle of $60^{\circ}$, standoff $H=20 \mathrm{~mm}$.

The blasted abrasives were collected from the dust chamber at the end of each experiment and the distributions of circular diameter $\left(D_{\text {Circular }}=2 \sqrt{\text { Area } / \pi}\right)$, and roundness $\left(R=\frac{4 \pi \text { Area }}{\text { Perimeter }^{2}}\right)$ were measured using an optical particle sizing system based on image analysis (Clemex PSA Research Unit, Clemex Technologies Inc., Longueuil, Quebec, Canada). The measured distributions were compared with the numerical simulation, as described in Section 4.4.1.

\subsubsection{Shadowgraphy}

During the experiments of Section 4.2.1 pulsed laser shadowgraphy was used to record images of particles just before, and after impacting the surface, in order to capture their fracture and fragmentation. Details on the apparatus and technique can be found in Ref. [95,99]. As shown in Fig. 4.2, an inclined target was fixed in front of the dust chamber between the diffuser and lens of camera. The relatively low abrasive mass flow rate (Section 4.2.1) ensured that there was no interaction between the particles in flight or during the impact phase and there were no more than two particles in any of the shadowgraphy images. 


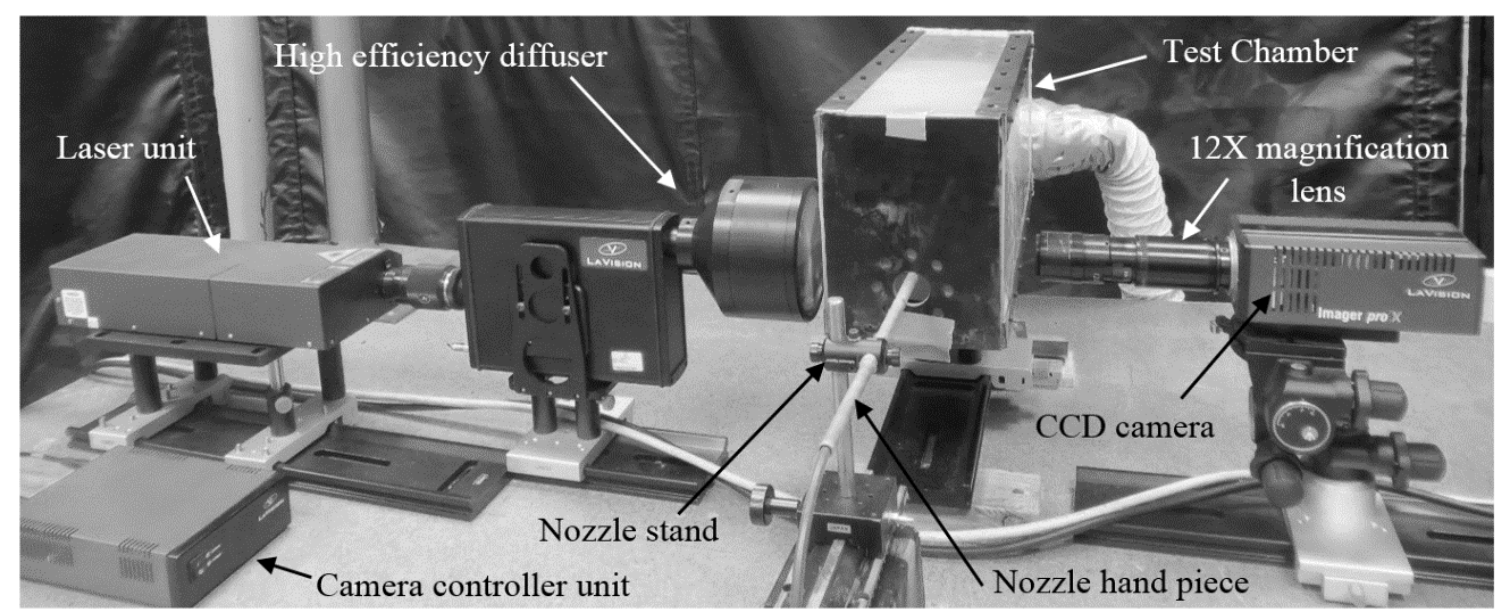

(a)

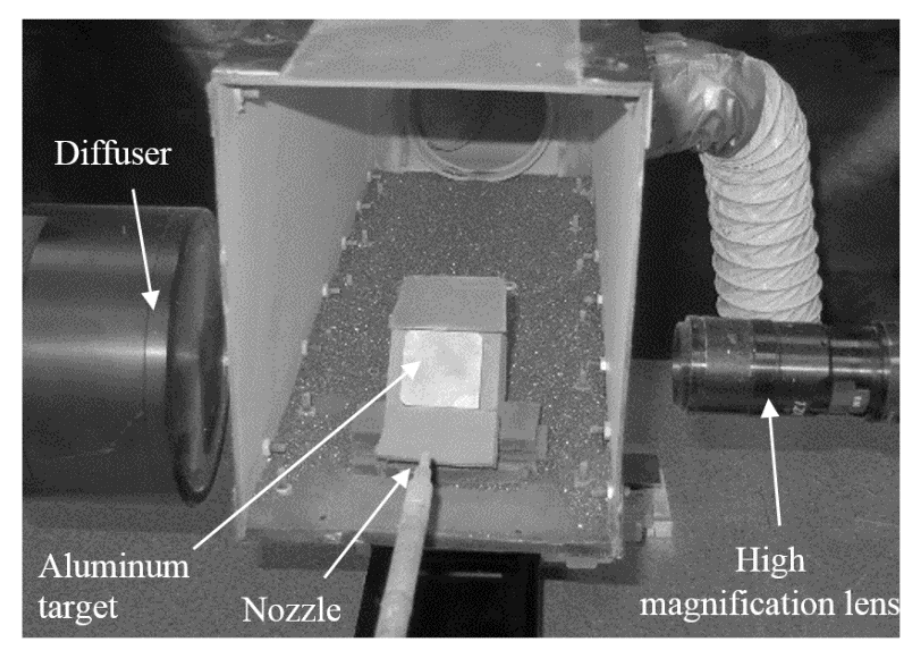

(b)

Figure 4.2 Experimental set up used for shadowgraphy experiments: (a) Shadowgraphic measurement system; (b) inclined target inside test chamber.

It was found that for an average particle velocity of $\sim 100 \mathrm{~m} / \mathrm{s}$, and a camera field of view of $4.5 \times 3.5 \mathrm{~mm}$, a $15 \mu \mathrm{s}$ delay between successive laser pulses ensured that multiple images before and immediately subsequent to impact could be obtained. Figure 4.3 shows typical images of particle impact fragmentation captured using the system. Using this technique, images of a total of more than 1500 particles were captured and used to measure the distributions of the resultant velocity of the abrasives before, and of the fragments, after impact. As expected, the fragments tended to rebound with a shallower angle than the incident, as will be discussed in detail in Chapter 
5. 100 images of the particles before impact, taken at random, were also utilized for generation of the particle geometries as discussed in Section 4.3.2.1.

Impact

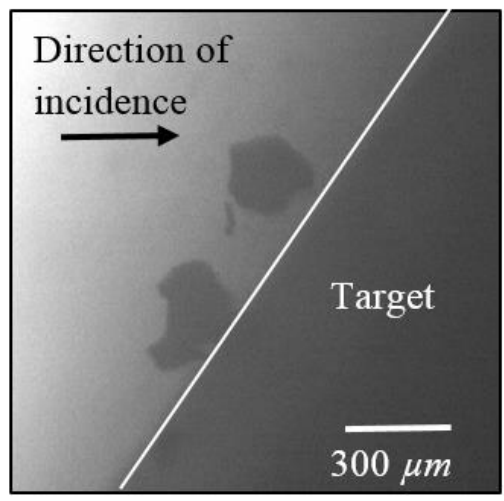

(a)
Rebound

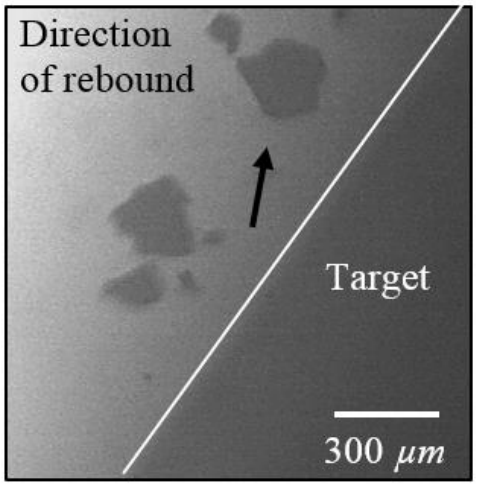

(b)

Figure 4.3 Typical shadowgraphy image showing the fracture and fragmentation of two typical particles impacting at a $60^{\circ}$ incidence angle and $100.3 \mathrm{~m} / \mathrm{s}:$ (a) before impact (b) after impact.

\subsection{Numerical simulation}

The numerical models, implemented in LS-dyna Version 971 (Livermore Software Technology Corporation, Livermore, CA, USA), focused on simulating the kinematics, and fracture and fragmentation of particles impacting the aluminum alloy target. Models were constructed for 100 particle impacts chosen randomly from the shadowgraphy images of the incident particles described in Section 4.2.2.

\subsubsection{Discretization and modeling of target material}

Previous work $[111,112,154]$ involved the numerical modelling of the solid particle erosion of the same target using the same particles as present, but did not consider particle fracture. As in that work, the smoothed particle hydrodynamic (SPH) technique with an SPH particle spacing of $5 \mu \mathrm{m}$ was used to model the Al6061-T6 target material, in order to avoid the mesh distortion problems associated with finite element analysis of such a large deformation problem. As before $[111,112,154]$, the Johnson-Cook constitutive $[115,116]$ and damage $[117,119,120]$ models, the Mie-Gruneisen equation of state [114,120], and the Cowper-Symonds model for strain rate 
sensitivity [118] were implemented to describe the target material behaviour. The renormalization approximation technique was utilized as the particle approximation method.

\subsubsection{Modeling of particles}

\subsubsection{CAD representation of particles}

Earlier numerical simulations by the present authors aimed at predicting erosion and particle embedment used collections of particle geometries with four vertices to represent the distribution of particle shapes in an abrasive powder [111, 154]. The present work, however, required a more refined approach in order to obtain the actual geometries of the impacting particles directly from the shadowgraphic images. Therefore, a new particle generation scheme was developed, based on the analysis of high resolution shadowgraphy pictures of the particles in the abrasive jet flow (Fig.4.4a) using PSA Professional Research Particle Size and Shape Image Analysis software (Clemex Technologies Inc., Longueuil, Quebec). The new methodology can be summarized as:

(i) The skeleton point representation (a one pixel wide list of the coordinates of the periphery) of the image of the particles in flight (Fig 4.4a) were obtained (Fig. 4.4b) using a routine developed for the Clemex software. Edge detection was used to detect the particles in the shadowgraphy images, whose areas were then assigned a typical color (bitplane 1). Then, a duplicate copy of the particle was generated and assigned to a second bitplane (bitplane 2). Using the "Erod" command in the Clemex software, one pixel was removed at each peripheral point of the area in bitplane 2 , i.e. bitplane 1 is one pixel larger than bitplane 2. The skeleton line representation was then obtained by Boolean subtraction of bitplane 2 from bitplane 1. Finally, the Clemex "Disconnect" operation was used to separate each individual pixel point from the line representation, and the coordinates of each skeleton point (pixel) were then exported to an output file.

(ii) The coordinates of the skeleton points were imported to computer aided design (CAD) modeling software (Solid Works 2014, Dassault Systems SolidWorks Corp.). The twodimensional (2D) particle geometries were created by connecting every two successive points along the perimeter (Fig. 4.4c) and generating a planar surface. 
(iii) The 2D particle geometries from (ii) were extruded in the third dimension to create the 3D geometry (Fig. 4.4d). The distance of extrusion was based on measurements of particle thickness distribution from Chapter 2. As discussed in Chapter 2, the utilized particles had flaky shapes, thus justifying the uniform extrusion.

(iv) The 3D particle geometries were saved in IGES format and imported into LS-Dyna, where they were meshed using tetrahedral finite elements (Fig. 4.4e) and used for generation of the impact model in the LS- Dyna Keyword format using the EFG formulation, as described in section 4.3.2.2.

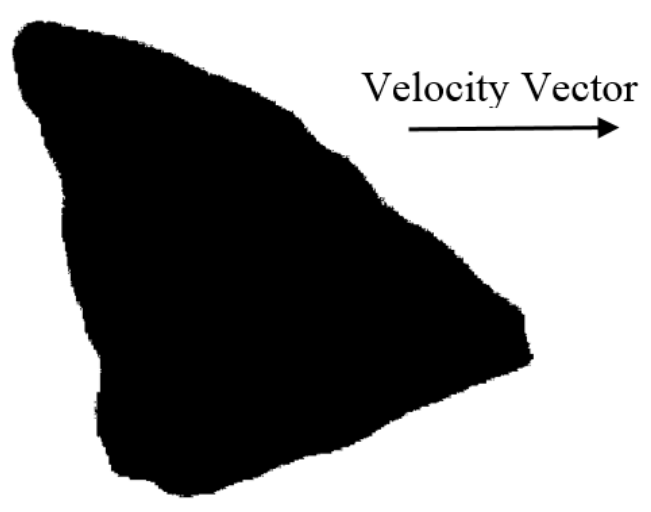

(a)

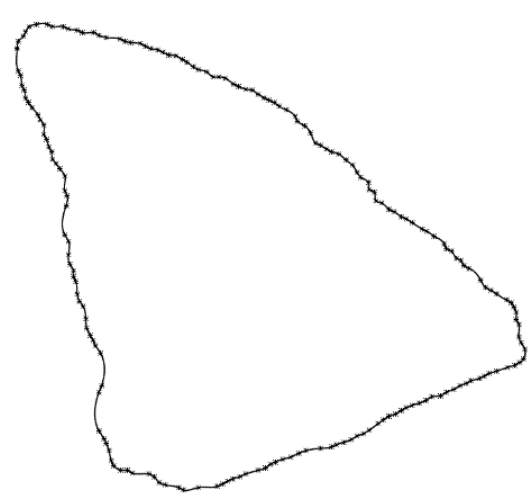

(c)

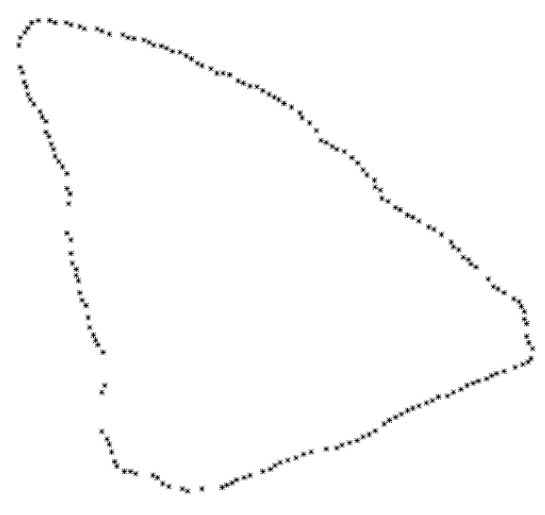

(b)

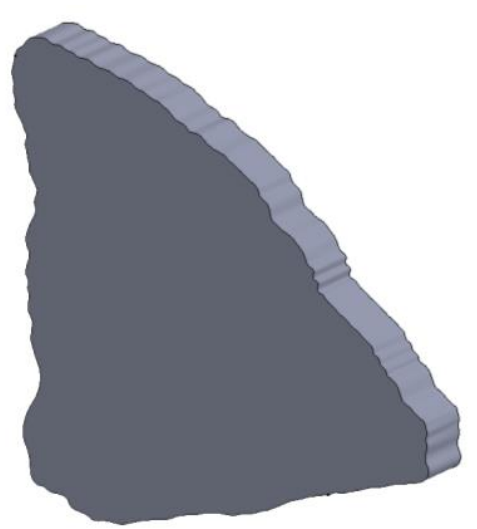

(d) 


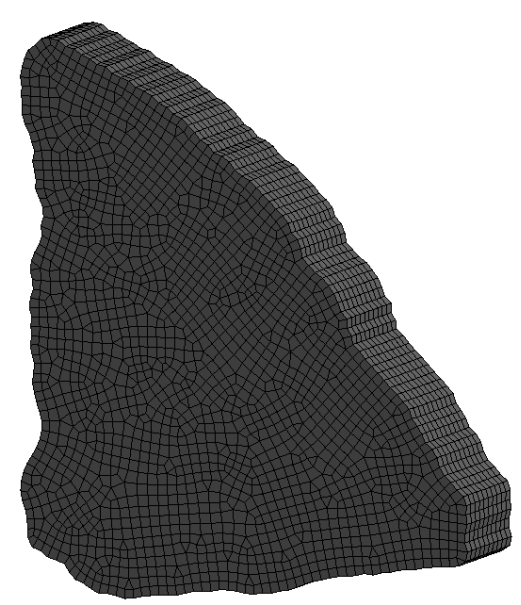

(e)

Figure 4.4 Particle geometry generation. (a) shadowgraphic image of a particle in flight (b) skeleton point representation (c) planar area (d) generated volume (e) meshed FE representation of the particle.

This method of particle generation not only resulted in geometries that were closer to the actual geometries than any other method developed thus far, but also had the advantage that the particle orientation in flight was automatically reflected in the resulting geometry.

\subsubsection{Section formulation for $\mathrm{SiC}$ abrasives}

The EFG solid element formulation (EQ.41) in the *SECTION_SOLID_EFG card of LSDYNA was used in modelling the fracture of the particles The full transformation method in the 3D Solid EFG card was used for treatment of essential boundary conditions. The EFG default fracture method (Local boundary integration), was used as the "domain integration method" (IDIM) [120]. The moving-least-square approximation technique was chosen as the principle formulation to generate the numerical discretization. The EFG implementation in LS-DYNA with the Automatic_Node_To_Surface contact card detects and recognizes the meshless geometry of the modeled parts, and the appropriate contacting surfaces, by implementing automatic sorting of background elements. 


\subsubsection{Johnson-Holmquist material model for $\mathrm{SiC}$ particles}

As discussed in section 4.1, the Johnson-Holmquist (JH-2) constitutive model (material 110, *MAT_JOHNSON_HOLMQUIST_CERAMICS in LS-Dyna) [71] appears to best represent the behavior of ceramics, and was therefore adopted to model the SiC particles. The JohnsonHolmquist constitutive model contains three major parameters; i.e., strength, which defines the strength of the undamaged and damaged ceramic, pressure which describes the relation between pressure and volume, and damage, representing the failure criterion in the context of change from an intact to a damaged mode [155]. The JH-2 material model considers the residual strength of the ceramic material after damage and also incorporates the bulking of the loaded ceramic.

In general, the failure of ceramics under impact is based on the birth and growth of microcracks which accumulate as damage and ultimately result in fracture. The compressive stress wave generated upon impact propagates in the radial direction from the impact point [77]. Crack initiation begins when the compressive stress becomes greater than the dynamic strength of the ceramic. Accumulation of such cracks results in fracture of the ceramic that propagates at the elastic wave velocity. When the compressive wave reaches the free boundary of the material, it is a reflected as a tensile wave that may cause additional cracking when the magnitude of the tensile stress becomes greater than local material strength.

Development of highly strained zones results in growth of damage and reduction of the strength of the damaged ceramic. The $\mathrm{JH}-2$ model incorporates a state (damage) variable to determine the failure of an infinitesimal volume of the ceramic. LS-Dyna applies the material constitutive model through an iterative algorithm to correlate the physical changes in the loaded ceramic such as the induced strain to the consequent variations in the stress [155]. Damage of the material in compression is accumulated if the magnitude of the deviatoric stress is greater than a specific (critical) value and is reflected as an increase in the damage variable $(D)$ varying between 0-1.0 (the plastic strain). In other words, the strength of the material at each time step is calculated based on the strength of the intact and damaged material. It is noted that sudden brittle fracture failure under tensile loading can occur when the material's load capacity (tensile strength) is reached.

In the $\mathrm{JH}$ formulation summarized below, the variables marked by an asterix (*) are normalized. For example, stresses and pressures are normalized with respect to the equivalent stress $\left(\sigma_{h e l}\right)$ and pressure $\left(P_{h e l}\right)$ calculated at Hugoniot elastic limit $(h e l)$ as follows [120]: 
$P_{h e l}=K_{1} \mu_{h e l}+K_{2} \mu_{h e l}{ }^{2}+K_{3} \mu_{h e l}{ }^{3}$

$\sigma_{\text {hel }}=1.5\left(\right.$ hel $\left.-P_{\text {hel }}\right)$

where $K_{1}, K_{2}, K_{3}$ are the first, second and third pressure coefficients and $\mu_{h e l}$ is calculated iteratively as,

hel $=K_{1} \mu_{\text {hel }}+K_{2} \mu_{\text {hel }}{ }^{2}+K_{3} \mu_{\text {hel }}{ }^{3}+\frac{4}{3} G \frac{\mu_{\text {hel }}}{1+\mu_{\text {hel }}}$

where $G$ is the shear modulus. The instantaneous strength of the ceramic material is then calculated as [120],

$\sigma^{*}=\sigma_{i}^{*}-\bar{D}\left(\sigma_{i}^{*}-\sigma_{f}^{*}\right)$

where $\bar{D}$ is the damage factor, and $\sigma_{i}^{*}, \sigma_{f}^{*}$ are the normalized magnitudes of the strength of the intact and damaged material, respectively, calculated as,

$\sigma_{i}^{*}=\bar{A}\left(P^{*}+T^{*}\right)^{N}(1+\bar{C} \ln \dot{\varepsilon})$

$\sigma_{f}^{*}=\bar{B}\left(P^{*}\right)^{M}(1+\bar{C} \ln \dot{\varepsilon})$

where $\bar{A}, \bar{B}$ and $\bar{C}$ are the intact normalized strength parameter, damaged normalized strength parameter, and strength parameter (for strain rate dependence) respectively. $M$ and $N$ are damaged and intact, strength parameters, respectively and $T^{*}, P^{*}$ are the maximum normalized tensile strength and the normalized hydrostatic pressure, respectively. The hydrostatic pressures in the intact ceramic under compression and tension are calculated as,

$$
\begin{array}{lc}
P=K_{1} \bar{\mu}+K_{2} \bar{\mu}^{2}+K_{3} \bar{\mu}^{3}+\Delta P_{n+1} & \text { (Compression) } \\
P=K_{1} \bar{\mu} & \text { (Tension) }
\end{array}
$$

where $\bar{\mu}$ is a measure of the change in volume

$\bar{\mu}=\frac{\rho}{\rho_{0}}-1$

$\rho_{0}, \rho$ are the initial and instantaneous densities of the material, respectively. 
Knowing the present magnitude of strain and the time step, the accumulated strain and effective strain rate are calculated at each step. The accumulated damage is

$\bar{D}=\sum \frac{\Delta \varepsilon^{p}}{\varepsilon_{f}^{p}}$

where $\Delta \varepsilon^{p}$ represents the increase in plastic strain and $\varepsilon_{f}^{p}$ is the plastic failure strain. The magnitude of the plastic strain resulting in damage to the ceramic under a typical pressure is calculated as

$\varepsilon_{f}^{p}=\bar{D}_{1}\left(P^{*}+T^{*}\right)^{\bar{D}_{2}}$

where $\bar{D}_{1}$ and $\bar{D}_{2}$ are constants.

Onset and development of damage is accompanied by an increase in pressure with a parameter $\beta$ (between 0 and 1) representing the proportion of the elastic energy which is transformed to pressure (hydrostatic potential energy) as calculated by LS-Dyna. More details on this can be found in LS-Dyna theory manual [155]. The JH parameters used for the SiC particles are available in the literature [156] and are summarized in Table 4.1.

Table 4.1 Material constants for SiC ceramic [156].

\begin{tabular}{|l|c|}
\hline $\bar{A}$ (normalized strength parameter for undamaged material) & 0.96 \\
\hline $\bar{B}$ (normalized strength parameter for damaged material) & 0.35 \\
\hline $\bar{C}$ (Strength parameter for strain rate dependence) & 0.0 \\
\hline$M$ (strength parameter for damaged material) & 0.0 \\
\hline$N$ (strength parameter for undamaged material) & 1.0 \\
\hline$E P S I$, Ref. Strain rate & 0.37 \\
\hline $\bar{T}$, Maximum tensile strength $(G P a)$ & 0.8 \\
\hline Maximum normalized failure strength (SFMAX) & 14.567 \\
\hline$H E L$, Hugoniot elastic limit $(G P a)$ & 5.9 \\
\hline$P H E L$, Pressure component at the Hugoniot elastic limit $(G P a)$ & 13.0 \\
\hline$T H E L$, HEL Strength $(G P a)$ & 0.48 \\
\hline $\bar{D}_{1}$ (Parameter for plastic strain to cause damage) & 0.48 \\
\hline $\bar{D}_{2}$ (Parameter for plastic strain to cause damage) & \\
\hline
\end{tabular}




\begin{tabular}{|l|c|}
\hline$K_{1}$ (First pressure coefficient) & 204.785 \\
\hline$K_{2}$ (Second pressure coefficient) & 0 \\
\hline$K_{3}$ (Third pressure coefficient) & 0 \\
\hline$\beta$, (Fraction of elastic energy loss converted to hydrostatic energy) & 1.0 \\
\hline$G$, Shear Modulus $(G P a)$ & 183 \\
\hline$\rho$, Density $\left(\frac{K g}{m^{3}}\right)$ & 3163 \\
\hline
\end{tabular}

\subsubsection{Modeling of fracture}

The JH material described in section 4.3.2.3 characterizes the damage, stresses and strains in the $\mathrm{SiC}$ ceramic material but does not alone allow for actual formation of cracked surfaces. For cracked surfaces to form (i.e. separation of the EFG elements representing the particle), criteria for generation of the cracked surfaces (a 'failure criterion') must be specified.

Failure strain is a widely used parameter to model damage and fracture of particles in literature [76]. To gain an initial insight into the magnitudes of strains and the possible fracture patterns generated during these impacts, the impacts of several particles were simulated with the failure criteria (FS) in JH material model set to zero. This meant that, while no elements would be eliminated from the modeled particle (i.e. no actual separation of material occurred), elements with sufficient damage to be considered 'failed' by the model, would be assigned zero stress. It was found that there were clear patterns of failed elements (corresponding to zero stress), and as shown in the example, these elements had maximum strain, between $0.75 \%-1.25 \%$ (Fig.4.5), values that are consistent with the failure strain for $\mathrm{SiC}$ particles found in literature. For example, Ayyar et al. [157] reported that the fracture of $\mathrm{SiC}$ particulates occurred at a failure strain of about 0.64-1\%, and Prewo [158] found the failure strain of $\mathrm{SiC}$ to be equal to $1 \%$. Based on this initial analysis, a critical failure strain value of $1 \%$ was used in the present work. 

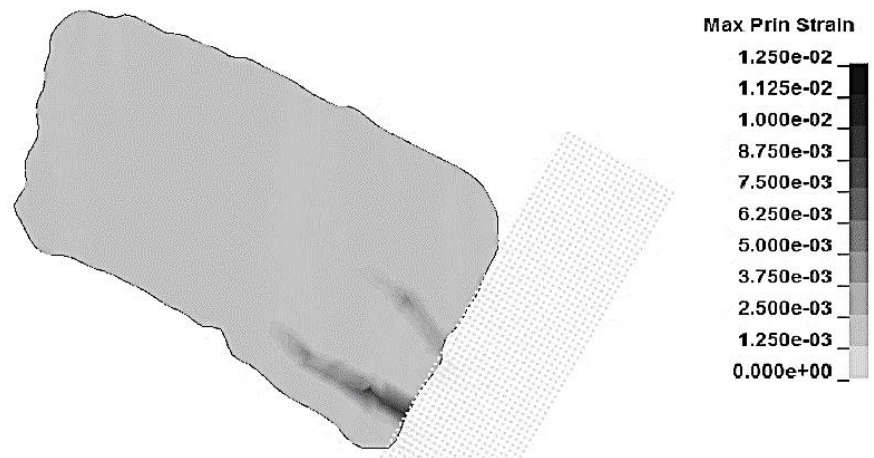

(a)
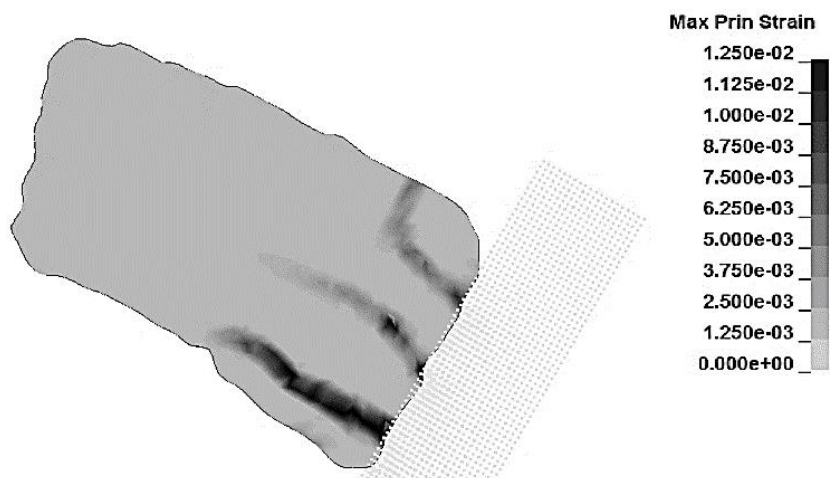

(b)

Figure 4.5 Contour plot of maximum principle strain for an impacting particle at (a) $t=0.1 \mu s$ (b) $t=0.2 \mu \mathrm{s}$

Two techniques for modelling the separation of material (fracture) were compared. The first utilized the JH failure parameter based on a critical failure strain. In other words, the elements in the particle corresponding to fracture were eliminated once the plastic strain was greater than the user defined value for failure strain (FS), assumed as 1\%. However, it was found that this approach resulted in excessive and unrealistic erosion at the tip of some of the particles, and, in some cases, the loss of contact between the particles and the SPH target. In order to prevent such contact loss, the contact between the slave and master parts must be redefined and continue on the 
new surface generated by the interior elements that are exposed due to fracture. LS-Dyna had difficulties redefining these contacts. It was found, however, that these difficulties could be eliminated by using the Mat-add-erosion failure criterion in LS Dyna, which eliminates the failed element from the calculations based on a user-defined criterion. The criterion applied in the *MAT_ADD_EROSION card for all further considered models was that the maximum principle strain at failure be equal to $1 \%$. The simulated fracture and fragmentation of a typical particle upon impact is shown in Fig. 4.6.

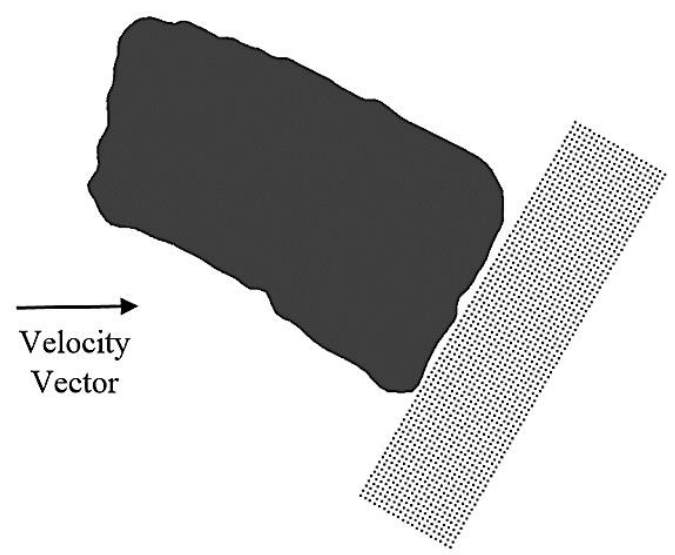

(a)

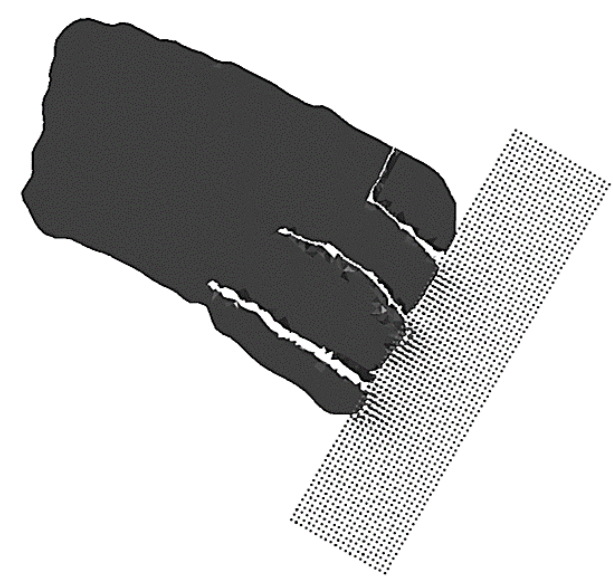

(c)

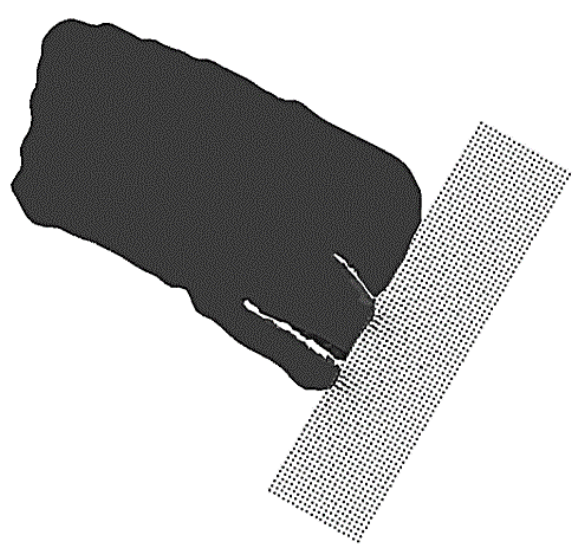

(b)

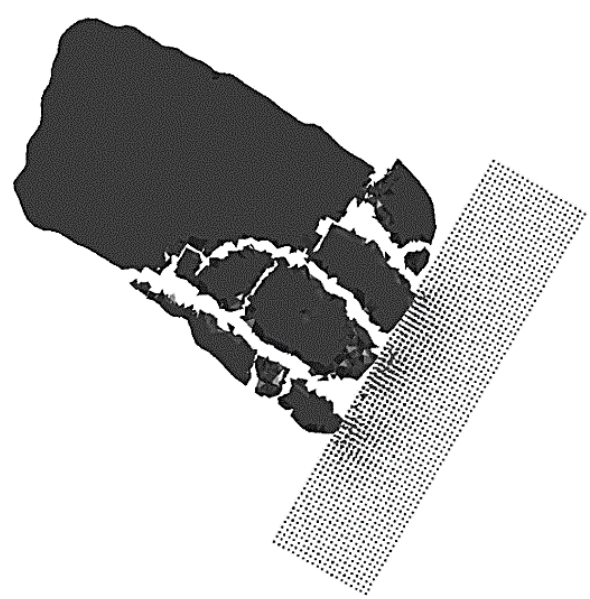

(d)

Figure 4.6 Crack initiation and propagation and fracture of particles at $V=120 \mathrm{~m} / \mathrm{s}$, impact
angle $=60^{\circ}$
(a) $t=0$
(b) $t=0.1 \mu \mathrm{s}$
(c) $t=0.15 \mu \mathrm{s}$
(d) $t=0.5 \mu \mathrm{s}$ 
In order to compare the measured size and shape of the abrasives retrieved from the erosion experiments with their corresponding predicted values, the geometrical characteristics of the fractured particles from the model were analyzed using the same PSA Professional Research Particle Size and Shape Image Analysis software (Clemex Technologies Inc., Longueuil, Quebec) that was also utilized in the measurements of the actual retrieved fragments described in section 4.2.1.

\subsection{Results and discussion}

Utilizing an Intel quad-core Core ${ }^{\mathrm{TM}} \mathrm{i7}-3770 \mathrm{CPU}$ with $12 \mathrm{~GB}$ of RAM, simulation of each 0.5 $\mu s$ of real time typically took approximately 2 hours.

As explained in more detail in Ref. [32,111,114,154], drawbacks of the SPH formulation include tensile instability and lack of neighboring nodes, both of which may influence the stability of the solution, and the accuracy of results. Changes in the magnitude of the energies during the simulation derived in post-processing stage can be used to assess these effects. In other words, the sum of the internal, kinetic, and sliding energies was analyzed to ensure that significant artificial energy was not generated due to these effects. As an example, Fig. 4.7 shows that the total energy increased by only $0.47 \%$ during the impact, and in general for all simulations, the total energy increased by no more than $3 \%$ illustrating that the simulation was numerically stable. 


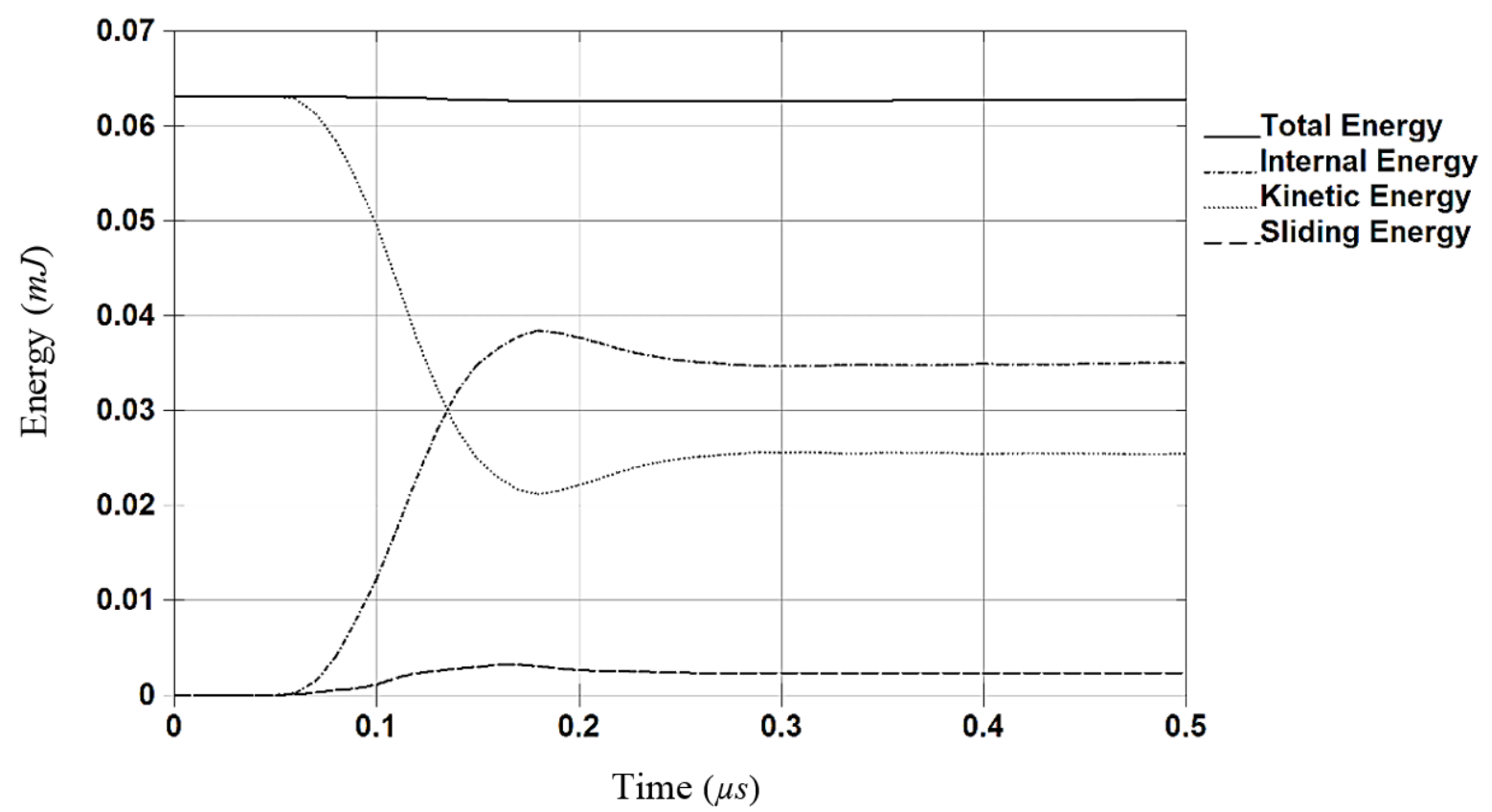

Figure 4.7 Changes in the magnitude of sliding, kinetic, internal and total energy during the impact and fracture simulation for a typical grit 60 particle at $100 \mathrm{~m} / \mathrm{s}$ incident velocity and $60^{\circ}$ impact angle.

The numerical simulation predicted that $53 \%$ of the particles fractured upon impact. The performance of the models were assessed by comparing measured and predicted fragment size, and particle rebound kinematics.

\subsubsection{Fragment geometry}

The measured (Section 4.2.1) average circular diameter of the abrasives before (Fig. 4.8a) and after erosion experiments (Fig. 4.8b) are $363 \mu \mathrm{m}$ and $156 \mu \mathrm{m}$, i.e. a more than two times reduction in size due to fracture. Figure $4.8 \mathrm{~b}$ compare the measured and predicted distributions of abrasive circular diameter after blasting, which were in reasonable agreement. The average circular diameter of the simulated particles after impact was $133 \mu \mathrm{m}$, compared to the measured value of $156 \mu \mathrm{m}$. This difference was in large part attributed to the fact that the CAD representation of the particles were generated based on shadowgraphic images of the particles, while the measurements of the fragments collected in the dust chamber were conducted using microscopic techniques. The particles are measured in all orientations while in flight using the shadowgraphic technique, but 
they tend to lay on their larger side under microscope, thereby introducing an orientation bias that tends to increase the measured size. An estimate of the strength of this bias can be obtained by comparing the average circular diameter of intact particles (before blasting) using both techniques. Figure 4.8a shows that using the microscopic technique results in an average particle size of 363 $\mu m$, which is 1.18 times larger than the $307 \mu m$ that resulted from using the shadowgraphic technique. With this in mind, the model predicted circular diameter of the particles was adjusted to include this microscope measurement bias by multiplying by 1.18. As shown in Fig.4.8b, the corrected predictions of average particle size were in excellent agreement with the measured $(<1 \%$ difference). Comparison between the circular diameter distributions of the intact and damaged particles (Fig. 4.8a and 4.8b) shows a clear change in the shape of distribution due to the fracture of the particles.

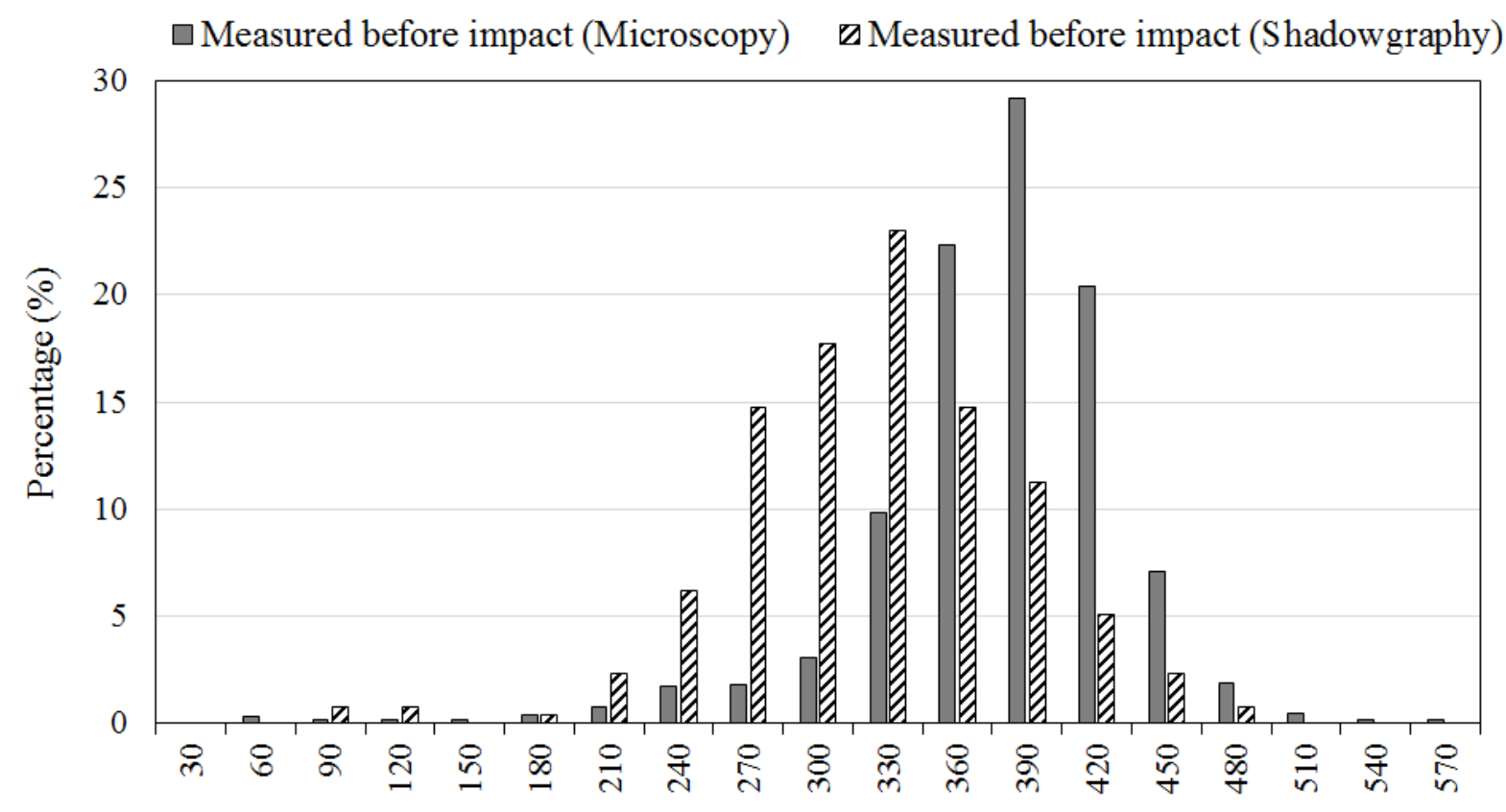

Circular Diameter $(\mu m)$

(a) 


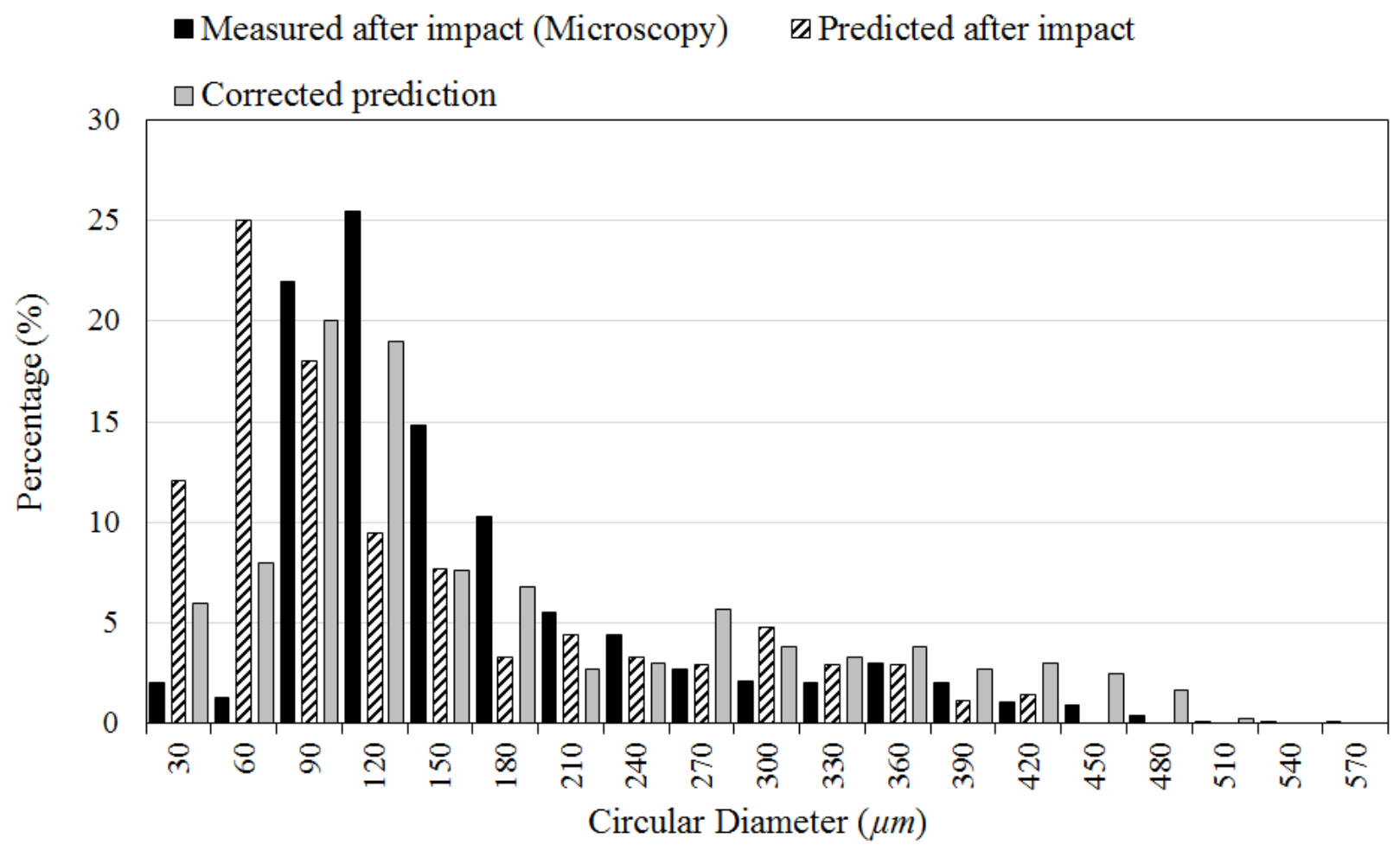

(b)

Figure 4.8 Distribution of particle circular diameter (a) before blasting, measured using microscope (average $=363 \mu \mathrm{m}$ ) and shadowgraphy (average $=307 \mu \mathrm{m}$ ), and $(\mathrm{b})$ after impact, measured using microscope (average $=156 \mu \mathrm{m}$ ), predicted from numerical simulation (average $=133 \mu \mathrm{m})$ and modified prediction to account for measurement bias (average $\left.D_{\text {Circular }}=157.5 \mu \mathrm{m}\right)$.

The measured distribution of particle roundness before impact, and the predicted and measured distributions of particle roundness after impact are shown in Fig. 4.9. The predicted and measured roundness distributions (Figs 4.9) are similar, although the simulated fragments were predicted to be slightly sharper (average roundness $=0.54$ ) than those measured (average roundness $=0.59$ ). The average measured particle roundness did not significantly change due to impact and fracture ( 0.6 before compared to 0.59 afterwards). 


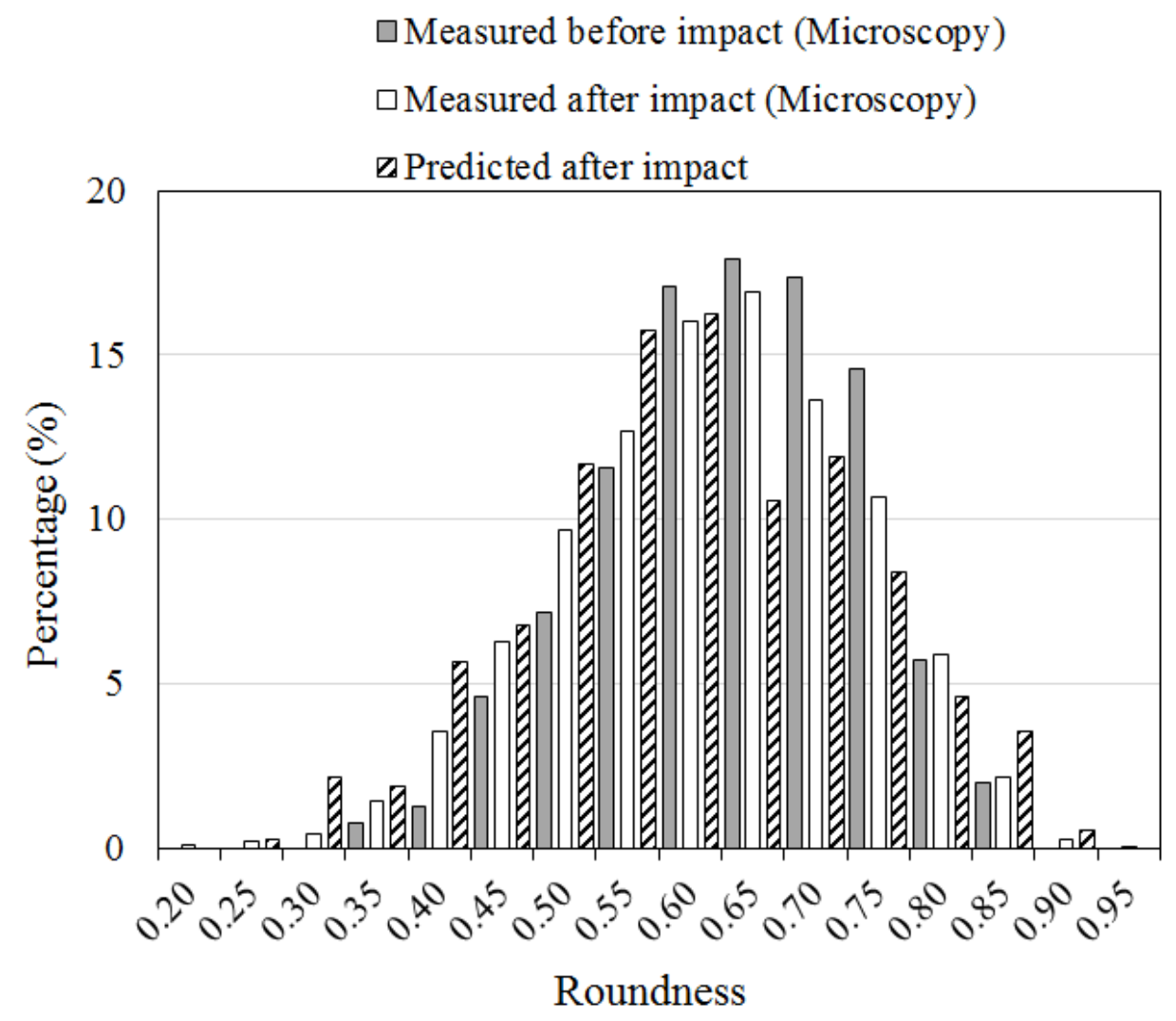

Figure 4.9 Distribution of particle roundness: Intact particles (average $=0.6$ ), predicted (average $=0.54)$, and measured after blasting (average $=0.59$ ).

In general, the particles were found to fracture either by cleavage, or by larger scale crushing. The abrasive residuals were found to be dissimilar and rough after impact at an oblique angle which is consistent with the findings of Salman et al. [49].

Figure 4.10 shows a direct comparison between the simulated and actual impact of a typical particle. The model predicted the same fracture pattern, number of fragments, and approximate fragment size as in the actual impact, thus lending confidence to an analysis of a larger number of impacts. 


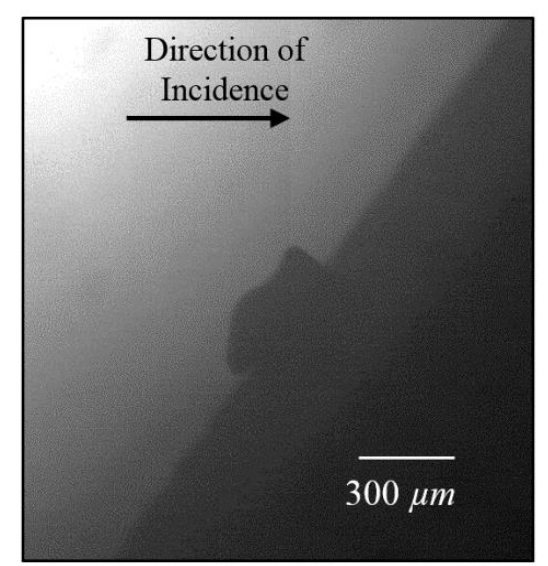

(a)

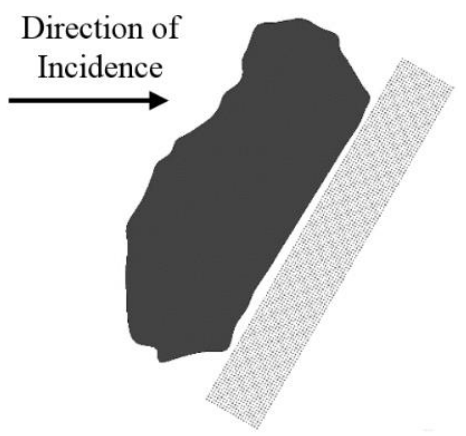

(c)

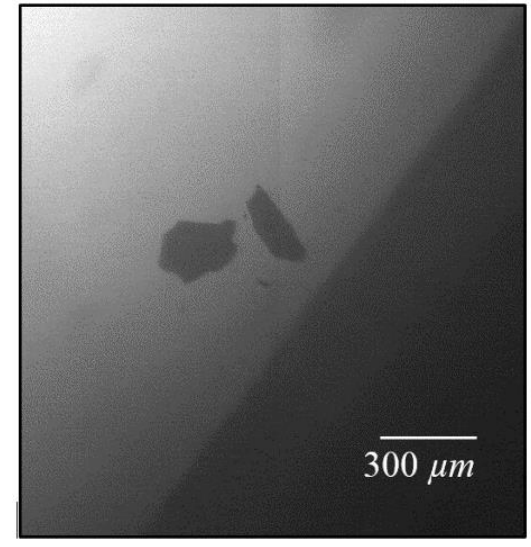

(b)

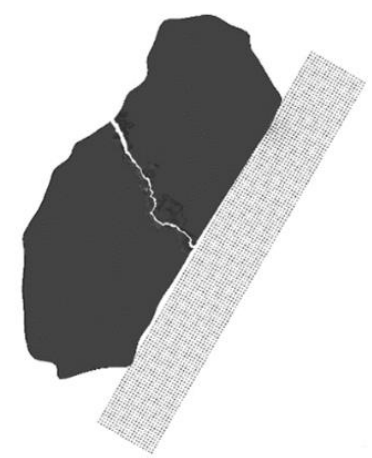

(d)

Figure 4.10 Typical images showing the impact and fragmentation of a particle (a) shadowgraphy image before impact, (b) shadowgraphy image after impact (c) modeled particle before impact and (d) modeled particle after impact.

\subsubsection{Particle rebound velocity}

Comparison of the distribution of the particle resultant incident and rebound velocities as measured using shadowgraphy in Fig. 4.11a and 4.11b shows that they follow approximately the same pattern, with averages that compare well. The ratios of the measured and predicted average rebounding velocities, to the average incident velocity (velocity ratio) were 0.34 and 0.36 , respectively. This implies that, almost $87 \%$ of the initial kinetic energy of the particles, i.e. in plastic deformation of the target and in particle fracture, was lost during the impact. Although a different target, particles, and velocities were considered, the present values are in the same range of 0.2 to 0.5 reported by Slikkerveer and in't Veld [135], for $23 \mu \mathrm{m}$ alumina particles impacting glass at $200 \mathrm{~m} / \mathrm{s}$. 
The fairly good agreement between predicted and measured rebound parameters shows the ability of the simulations to successfully predict impact kinematics, useful perhaps in future studies of the effect of collisions between incident particles and rebounding fragments under conditions of high particle flux.

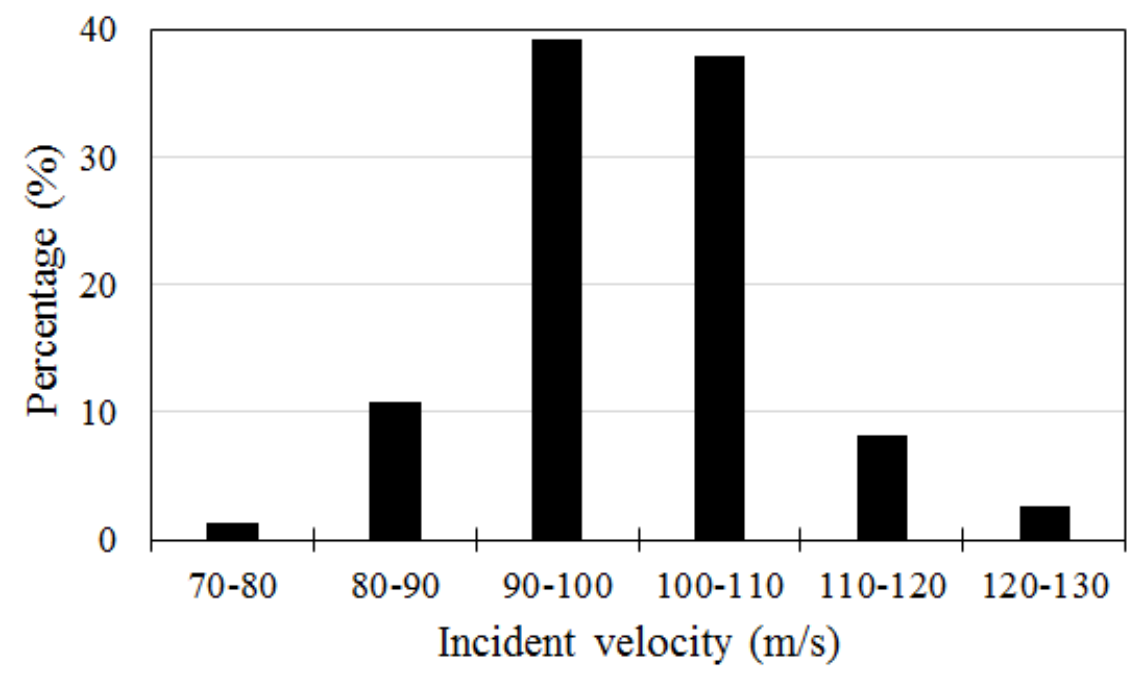

(a)

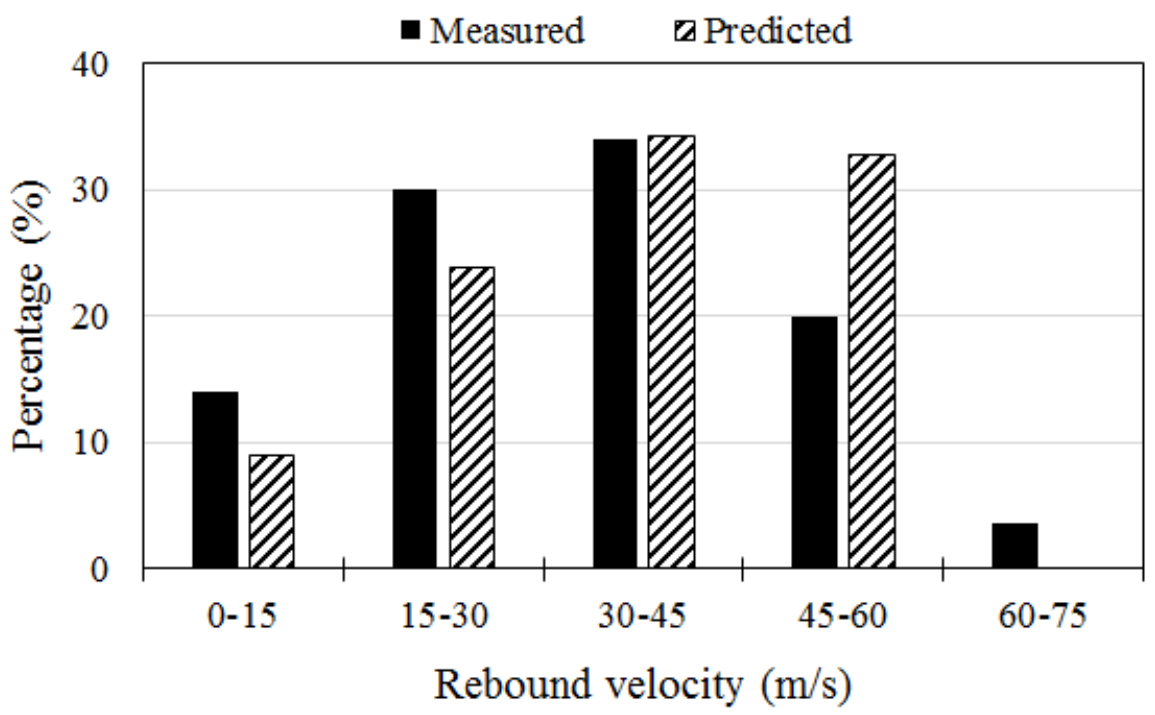

(b)

Figure 4.11 Distribution of (a) measured abrasive impact velocity (average $=100.3 \mathrm{~m} / \mathrm{s}$ ) (b) measured abrasive rebound velocity (average: $34.1 \mathrm{~m} / \mathrm{s}$ ) and predicted rebound velocity (average $36.1 \mathrm{~m} / \mathrm{s}$ ). 


\subsection{Summary}

Double pulsed shadowgraphy was used to, for the first time, capture the impact, fracture and fragmentation of grit $60 \mathrm{SiC}$ particles impacting a Al6061-T6 target at $\sim 100 \mathrm{~m} / \mathrm{s}$ and a $60^{\circ}$ impact angle. Analysis of the images and measurement of the particle fragments after impact revealed a marked change in the particle size distribution due to fragmentation upon impact. The shadowgraphic method also made it possible to measure the incident and rebound velocity of the particles and fragments. The average rebound velocity was typically 0.34 times lower than the average incident velocity. In other words, almost $87 \%$ of the initial kinetic energy of the launched particles was consumed during the impact process, mainly attributed to plastic deformation of the target, friction and the fracture of the particles.

A coupled EFG and SPH technique was utilized for the first time to simulate the impact and fracture of $\mathrm{SiC}$ particles on an aluminum alloy target. A novel algorithm was introduced to directly convert shadowgraphic images of particles in flight to 3D CAD geometries suitable for numerical modelling. The numerical models were found to accurately predict the measured particle average size ( $<1 \%$ difference), roundness $(9.2 \%$ difference) and rebound velocity (6\% average difference) as well as their distributions. Chapter 5 will use these models with further shadowgraphic measurements in order to further verify the model, and to shed light on the influence of process parameters such as abrasive size, incident velocity and impact angle on the likelihood and extent of particle fracture, and the resulting particle rebound kinematics for a typical solid particle erosion application. 


\section{Numerical and Experimental Investigation of the Influence of Process Parameters on Particle Fracture during Solid Particle Erosion}

This chapter is based on the following submitted paper

V. Hadavi, C. E. Moreno, M. Papini, Numerical and Experimental Analysis of Particle Fracture during Solid Particle Erosion, Part II: Effect of Incident Angle, Velocity and Abrasive Size, Wear (Article in Press)

\subsection{Introduction}

Fracture of abrasive particles during solid particle erosion processes is an important phenomena that may change the material removal mechanism, rebound velocity, angle and ultimately the erosion rate. The extent and likelihood of particle fracture depends on parameters such as the mechanical and geometrical characteristics of the target and particle, particle size and shape, hardness, toughness, incident velocity and angle. Chapter 4 presented and verified a numerical model that was shown to be appropriate for the prediction of particle fracture in a typical solid particle erosion process. The present work provides further experimental verification of the model, while using the results to study the effect of process parameters such as particle incident velocity, angle and size on the fracture of abrasives upon impact.

Incident velocity has been found to greatly affect particle fracture in solid particle erosion [48]. For example, Salman et al. [49] reported that, at a relatively low incident particle velocity of less than $9 \mathrm{~m} / \mathrm{s}, 5.15 \mathrm{~mm}$ alumina spheres impacting on steel target only elastically deformed, whereas at $25 \mathrm{~m} / \mathrm{s}$ and above, they all fractured. They also observed that the transition from undamaged particles to fragmentation occurred suddenly at a critical velocity, and that smaller particles fractured at a higher velocity than larger ones [49]. Sparks and Hutchings [51] studied the fragmentation of 125-150 $\mu \mathrm{m}$ silica abrasives upon impact on silicate glass ceramic and also found that likelihood of particle fracture was strongly proportional to incident velocity. For instance, they reported no particle fragmentation at $44 \mathrm{~m} / \mathrm{s}$ incident velocity and $30^{\circ}$ impact angle even after recycling the abrasives 5 times. There is also empirical evidence $[49,56]$ that the average 
fragment diameter decreases with increasing impact velocity, and that the fragment shape may also be influenced by velocity.

Impact angle has also been found to significantly affect the likelihood and extent of particle fracture. For example, the experiments performed by Salman et al. [49] showed that, at a constant velocity, the number of incident particles that did not fracture upon impact increased when the impact angle was shallower. They also reported that, for a given incident velocity, the number of unbroken abrasives at impact angles greater than $50^{\circ}$ was significantly lower than below $50^{\circ}$. Finally, they found a higher critical value of velocity was required for onset of particle fracture at lower impact angles. However, the transition from no fracture to fragmentation of all particles occurred much faster at higher impact angles compared to the more gradual trend observed at small impact angles [49].

Abrasive size has been found to affect the likelihood of fracture at a given velocity [159,160]. Salman et al. [49] conducted various single impact experiments using 3.14, 5.15 and $7.15 \mathrm{~mm}$ spherical $\mathrm{Al}_{2} \mathrm{O}_{3}$ particles at incident velocities under $35 \mathrm{~m} / \mathrm{s}$ and reported that the larger abrasives were more likely to fracture. They also reported that transition from full fracture to a no fragmentation mode occurred at a narrower range of velocities for larger particles than smaller ones [49]. Akbarzadeh et al. [50] studied particle fracture using 12 different types of target materials impacted by magnetite abrasives of $6.9 \mu \mathrm{m}$ and $30.4 \mu \mathrm{m}$ average diameters at 90 and 130 $\mathrm{m} / \mathrm{s}$ incident velocity at different impact angles. They found a higher degree of fracture and fragmentation for the larger abrasives, and also hypothesized that particle size would have a greater effect on fracture at a larger impact angle.

The dependence of fragmentation on particle size is likely linked to both the instantaneous target hardness, and the apparent particle fracture toughness. At a given incident velocity, particle fracture is also linked to particle size through changes in the kinetic energy available for creating fractures. Salman et al. [49] reported that the number of unbroken particles at the same velocity is higher for softer targets. Surface hardness may instantaneously change due to the generation of an impact-induced work-hardened layer [52-54], which may depend on particle size [50,55], which, in turn may influence the likelihood particle fracture, as discussed by Misra and Finnie [52] and Akbarzadeh et al. [50]. Particle size may also affect fragmentation due to the intrinsic differences in particle strength per unit volume. As suggested by Salman et al. [49] and Murugesh and Scattergood [56], this can be correlated to the larger number and size of flaws found in larger 
particles which, according to Harold [161] and Finnie [162] serve to reduce their brittle strength. Shipway and Hutchings [163] conducted extensive experiments on a sintered boron carbide ceramic target using silica, alumina and silicon carbide abrasives. They suggested that the silica abrasives of lower fracture toughness fractured before the boron carbide target, so that impact energy was consumed in fracturing the silica rather than the target, which resulted in erosion by small scale chipping. They also collected the silica particles after impact and reported that the silica fractured by cleavage, resulting in particles that were sharper than before impact.

Sparks and Hutchings [51] collected and re-used the fragmented silica and glass spheres after each abrasion phase on a glass-ceramic target. Their survey on recycling the abrasives illustrated that this process can result in progressive fragmentation of abrasives which can change the erosion condition at each stage. Their study showed that the erosion rate of a glass-ceramic target eroded by silica abrasives slightly improved while using the fractured particles after one cycle. However, using fragments after more than two cycles reduced the erosion rate. They suggested that the observed variation in erosion rate was due to the influence of changes in size, shape and aerodynamic condition of the fragments.

As discussed in Chapter 4, there is controversy regarding the correlation between particle fracture and erosion rate in literature. For example, Goodwin et al. [164] suggested that the piled up material could be eroded and separated by the radial wash of fragments, and Akbarzadeh et al. reported that the secondary impact of fragments may enhance the erosion [50]. However, abrasive breakage and fracture may also result in blunting of sharp particles that can decrease the overall erosion rate [139]. Moreover, the portion of the kinetic energy used for fragmentation of abrasives may not be available to erode the target, implying that the resulting erosion rate should be lower. These discussions of the effect of particle fracture on erosion rate have also been used to explain the apparent plateau $[48,162,165]$ or even reduction [53] in erosion that occurs when the particle size is increased above approximately 100-150 $\mu \mathrm{m}$. Increased interaction between incident and rebounding particles with increasing particle size, even if it does not lead to fracture, may also contribute to loss of energy that decreases the resulting erosion rate [50].

In summary, there have been a number of experimental observations regarding the effect of various process and material parameters on the extent of abrasive particle fracture due to impact and the resulting fragment geometry. However, most of these previous studies were performed in a time when modern computational and experimental tools did not exist. The present study 
demonstrates that double-pulsed laser shadowgraphic measurements and the computational model of Chapter 4 can be used as tools to more rigorously study the effect of particle size, impact angle and incident velocity on the extent of fracture, the fragment shapes and sizes, and their rebound kinematics.

\subsection{Experiments}

In order to analyze the influence of incident velocity, impact angle, and particle size on the extent of abrasive fracture, and to further experimentally verify a numerical model, jet impact experiments further to those presented in Chapter 4 were performed (Table 5.1). In all cases, shadowgraphy was used to measure rebound particle velocities and angles, and the distributions of size and shape of collected abrasive residues after impact were measured using the same microscopic technique as in Chapter 4. The geometrical characteristics of both the grit 60 and grit 120 abrasives are given in Table 5.2, and are based on measurements taken from an earlier study by the present authors [99].

Table 5.1 Process parameters using in shadowgraphy experiments. The last column indicates whether numerical models were also developed for a particular set of parameters. *Data in last row from Chapter 4.

\begin{tabular}{|c|c|c|c|c|c|}
\hline $\begin{array}{c}\text { Grit } \\
\text { Size }\end{array}$ & $\begin{array}{c}\text { Pressure } \\
(\mathrm{kPa})\end{array}$ & $\begin{array}{c}\text { Average Velocity } \\
(\mathrm{m} / \mathrm{s})\end{array}$ & $\begin{array}{c}\text { Impact } \\
\text { Angles }(\text { deg. })\end{array}$ & $\begin{array}{c}\text { Standoff } \\
\text { Distance }(\mathrm{mm})\end{array}$ & $\begin{array}{c}\text { Numerical } \\
\text { Modeled }\end{array}$ \\
\hline 120 & 160 & 100.1 & $60^{\circ}$ & 20 & YES \\
\hline 60 & 100 & 46 & $30^{\circ}$ & 20 & YES \\
\hline 60 & 100 & 46 & $60^{\circ}$ & 20 & NO \\
\hline 60 & 400 & 100.3 & $30^{\circ}$ & 20 & NO \\
\hline 60 & 400 & 100.3 & $60^{\circ}$ & 20 & YES* \\
\hline
\end{tabular}


Table 5.2 Distribution of various characterizing parameters for the utilized SiC particles [99].

\begin{tabular}{|c|c|c|c|c|c|c|c|c|}
\cline { 2 - 9 } \multicolumn{1}{c|}{} & \multicolumn{2}{c|}{ Area } & \multicolumn{2}{c|}{$D_{\text {circular }}$} & \multicolumn{2}{c|}{ Thickness } & \multicolumn{2}{c|}{ Roundness } \\
\hline $\begin{array}{c}\text { Grit } \\
\text { Size }\end{array}$ & $\begin{array}{c}\text { Mean } \\
\left(\mu m^{2}\right)\end{array}$ & $\begin{array}{c}\text { Standard } \\
\text { Deviation } \\
\left(\mu m^{2}\right)\end{array}$ & $\begin{array}{c}\text { Mean } \\
(\mu m)\end{array}$ & $\begin{array}{c}\text { Standard } \\
\text { Deviation } \\
(\mu m)\end{array}$ & $\begin{array}{c}\text { Mean } \\
(\mu m)\end{array}$ & $\begin{array}{c}\text { Standard } \\
\text { Deviation } \\
(\mu m)\end{array}$ & Mean & $\begin{array}{c}\text { Standard } \\
\text { Deviation }\end{array}$ \\
\hline 120 & 18507 & 5183 & 151.9 & 21.8 & 39.44 & 12.2 & 0.58 & 0.12 \\
\hline 60 & 107357 & 32629 & 362.9 & 70.6 & 94.9 & 31.6 & 0.6 & 0.11 \\
\hline
\end{tabular}

\subsection{Numerical simulations}

In order to evaluate the influence of impact angle, incident velocity and particle size on different aspects of particle fracture, new models in addition to those presented in Chapter 4 were constructed (Table 5.1). In all cases, as explained in Chapter 4, results from 100 models of impacts from the shadowgraphic incident data were presented. The study of the influence of particle size on fracture required the modelling of grit 120 particles. Since the shapes of grit 60 and grit 120 abrasives were very similar (Table 5.2, Roundness data), the geometries of the same 100 particles which had already been generated to represent grit 60 abrasives in Chapter 4, were scaled down to fit the measured size distribution of the grit 120 particles. In all cases, the resulting size and shape of the particles after blasting (intact plus fragmented particles), the ratio of rebound to incident velocity, and the rebound angles were analyzed and compared.

As explained in Chapter 4, differences in measurement technique used for model input (shadowgraphy) and fragment measurement (microscope) introduced a bias that caused size measurements made with the microscope to be approximately 1.18 times larger than with shadowgraphy. Therefore, all model-predicted particle sizes after impact were corrected for this bias, i.e. they were multiplied by the 1.18 factor so that they could be directly compared to measurements made with the microscope.

\subsection{Results and discussion}

\subsubsection{Further verification of numerical model for low impact angle and velocity}

In general, examination of the shadowgraphy images and numerical models revealed that particle fracture through both large scale crushing and cleavage. The numerical model developed 
for fracture of the grit 60 particles was experimentally verified in Chapter 4 for the most severe combination of process parameters used in this study, i.e. the conditions at which fracture is most likely to occur, at the highest $\left(60^{\circ}\right)$ impact angle and incident velocity $(100 \mathrm{~m} / \mathrm{s})$. As is shown in Figs. 5.1 and 5.2, the predictions of the model also match experimental ones reasonably well at the much less severe condition of a $30^{\circ}$ impact angle and $46 \mathrm{~m} / \mathrm{s}$ incident velocity. The predicted and measured particle size and roundness distributions after blasting are of similar shape, and the average predicted circular diameter and roundness are within $2 \%$, and $4 \%$, respectively of those measured.

As was found for the more severe condition in Chapter 4, the impacted particles and fragments were only slightly sharper than the intact abrasives. Comparison of the bimodal distribution of particle circular diameter under this less severe condition (Fig. 5.1), with the lognormal distribution at the more severe condition in Fig. 4.8b, shows that the process parameters have a strong influence on fragmentation. The less severe condition in Fig. 5.1 mainly resulted in small fractures at the particle tips, which led to the first peak in fragment size around 60-120 $\mu \mathrm{m}$, with the second peak occurring at fragment sizes only slightly smaller than the original particle size of $363 \mu \mathrm{m}$. On the other hand, the more severe condition in Chapter 4 led to a larger scale fracture of all the particles. The effect of impact velocity and angle will be discussed in more detail in Sections 5.4.3 and 5.4.4. 


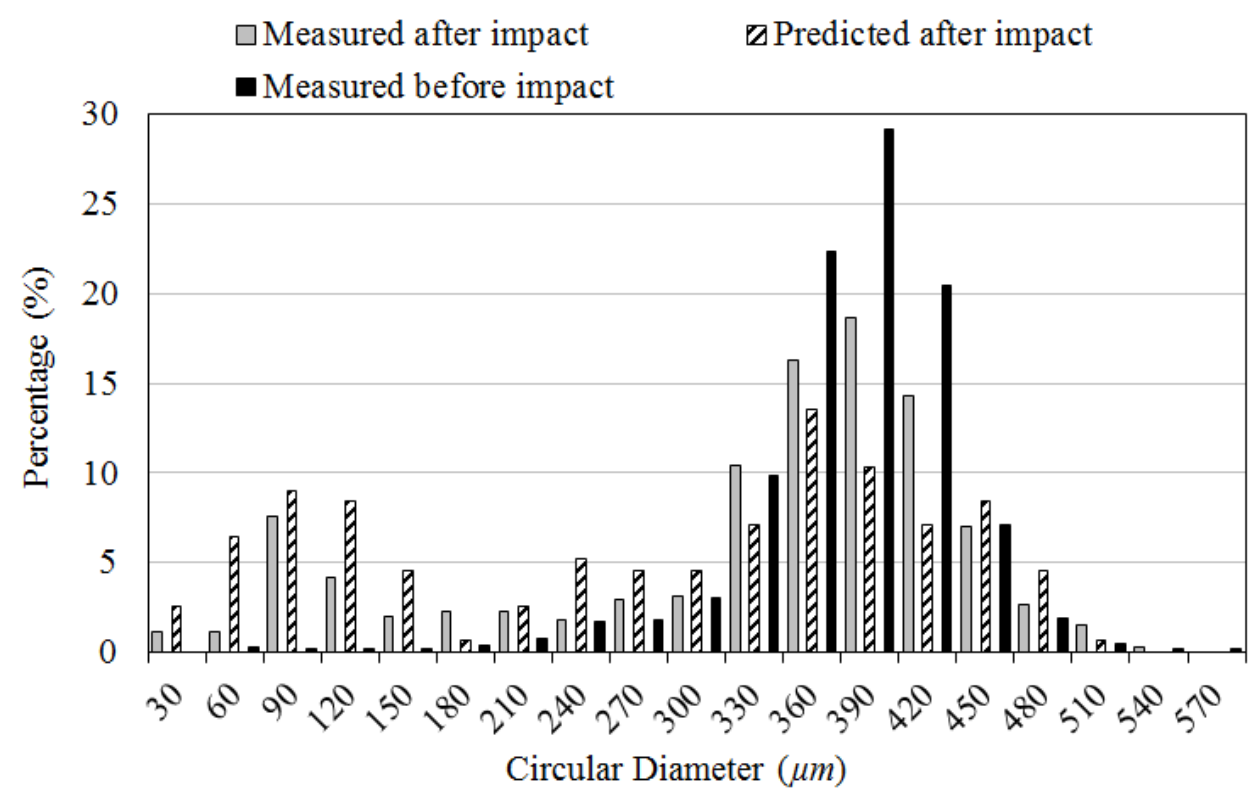

Figure 5.1 Predicted (average $=317 \mu \mathrm{m})$ and measured (average $=323 \mu \mathrm{m}$ ) size distributions of grit 60 particles after impact at $46 \mathrm{~m} / \mathrm{s}$ and a $30^{\circ}$ impact angle, compared to before impact $($ average $=363 \mu \mathrm{m})$.

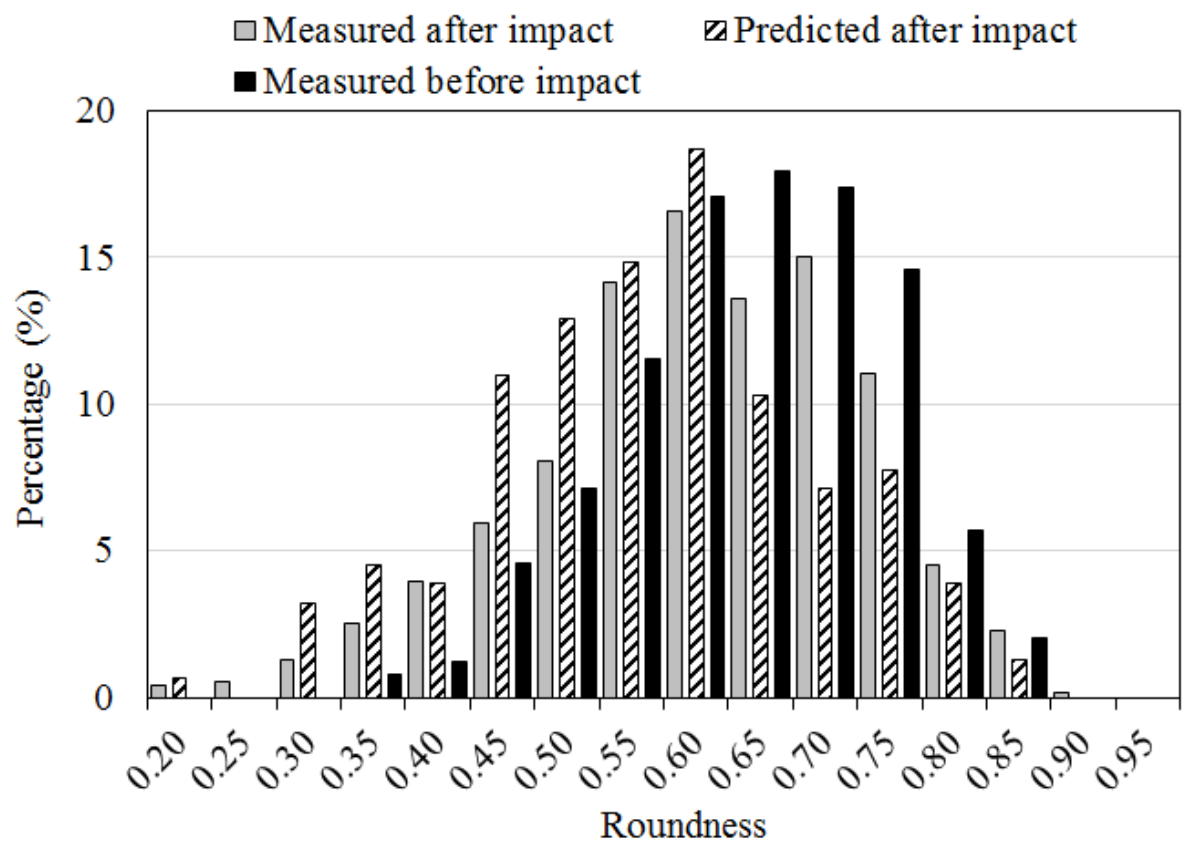

Figure 5.2 Predicted (average $=0.56)$ and measured (average $=0.58)$ roundness distributions of grit 60 particles after impact at $46 \mathrm{~m} / \mathrm{s}$ and a $30^{\circ}$ impact angle, compared to before impact (average $=0.6$ ). 
Figure 5.3 shows the distribution of predicted and measured $V x$ and $V y$ components of rebound velocity for the grit 60 particles at $30^{\circ}$ impact angle and $46 \mathrm{~m} / \mathrm{s}$. The measured and predicted rebound angles for $30^{\circ}$ impact angle at $46 \mathrm{~m} / \mathrm{s}$ vary between $5^{\circ}$ to $49^{\circ}$ (average $=27^{\circ}$ ) and $2^{\circ}$ to $59^{\circ}$ (average $=26^{\circ}$ ), respectively. Although the average values are very close, the predicted range is slightly wider than that measured. It can therefore be concluded that the kinematics of the fragments are also fairly well predicted by the model, making them suitable for use in, e.g., the assessment of particle interference effects. For example, refs [152,153] show that the probability of interference between incident and rebounding particles depends on the coefficient of restitution, and the 'stream density', a measure of the free space between particles in the incident and rebound jets. These kinematic quantities can all be predicted by the present model.

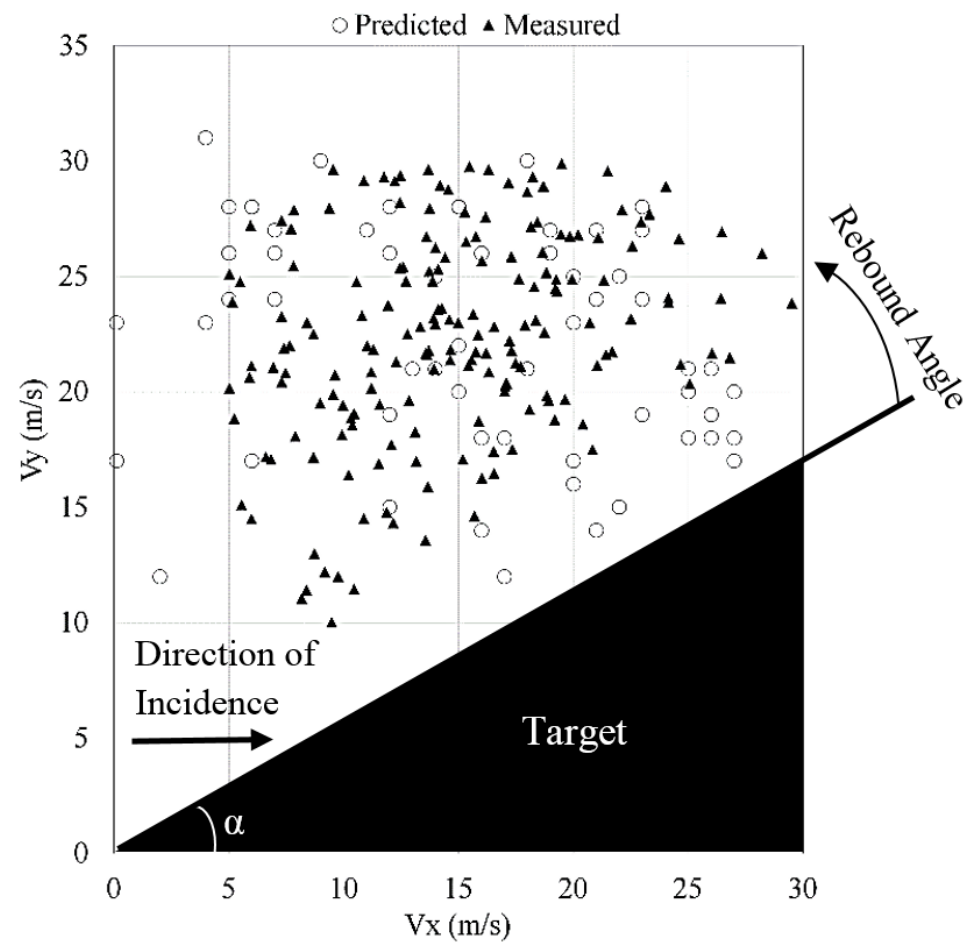

Figure 5.3 Distribution of predicted and measured velocity components for grit 60 particles at $V x=46 \mathrm{~m} / \mathrm{s}$ incident velocity and $30^{\circ}$ impact angle. 


\subsubsection{Relationship between average diameter of particles after impact and number of fractured particles}

A major advantage of the numerical models was that they allowed for tracking of individual particles through the impact and fracture process, so that accurate assessments of the proportion of launched particles that actually fractured could be obtained. In the past, such information could only be obtained by a series of tedious single macro-particle impacts where relatively large particles were individually measured, blasted, and the fragments collected. Even modern experimental tools such as shadowgraphy cannot be reliably used to speed up the process since they require fortuitous timing where images of the same particles before and after impact are obtained. In order to discuss the effects of the process parameters on the propensity for fracture, it was of interest to determine whether the average particle diameter after impact, a relatively simple measurement, could be related to the number of incident particles that fractured. Indeed, Fig. 5.4, based on the numerical results for the cases considered in Table 5.1, shows that the ratio of the average particle diameter after to before impact decreases monotonically with an increase in the percentage of incident particles that fractured. In other words, a lower average particle size after blasting indicates that a greater proportion of launched particles fractured. Therefore, further assessments of propensity for particle fracture were based on the size of the particles after impact.

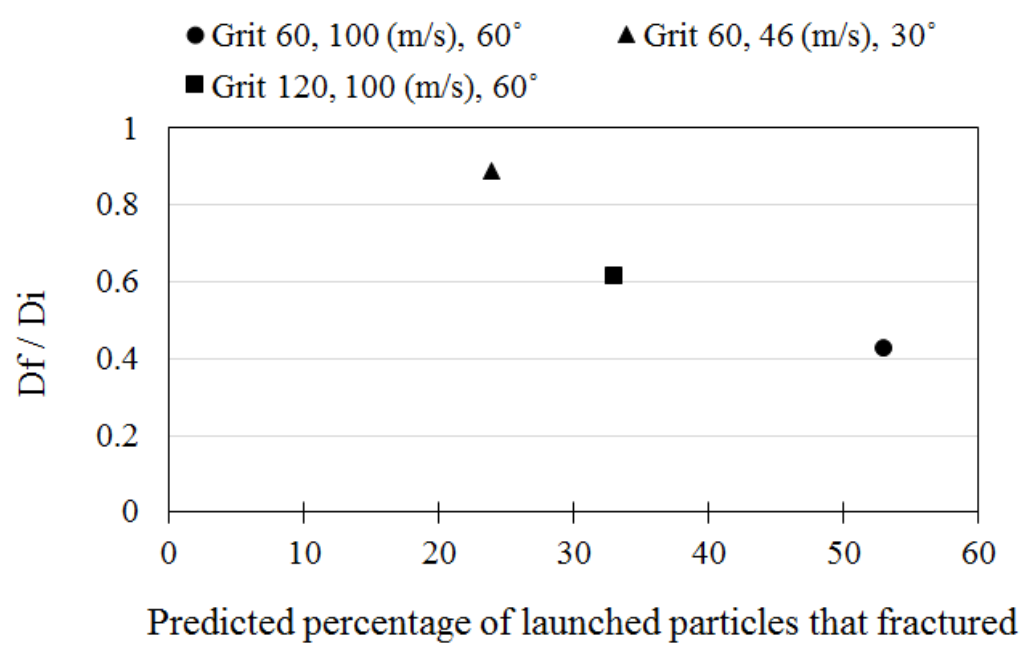

Figure 5.4 Relationship between measured normalized particle size after impact and predicted percentage of launched particles that fractured. $D f$ and $D i$ indicate measured average particle diameters before and after impact, respectively. 


\subsubsection{Influence of impact velocity and threshold for particle fracture}

Comparison between the measured size distributions of the collected grit 60 particles after impact at $30^{\circ}$ for incident velocities of 46 and $100.3 \mathrm{~m} / \mathrm{s}$ (Fig. 5.5a) illustrates that the higher velocity resulted in far smaller fragments; i.e., the average circular diameter of the particles after blasting decreased by 1.54 times. This is in agreement with the findings of Salman et al. [49] and Murugesh and Scattergood [56] who reported that the extent of particle fracture increased with incident velocity and that particles would be broken into more fragments as the velocity increases. At a larger impact angle of $60^{\circ}$ (Fig. 5.5b), the effect of velocity was even greater, with an almost twofold decrease in average circular diameter.

As mentioned in section 5.4.1, the process parameters strongly influence the distribution of fragment circular diameter. Figure 5.5 illustrates that increases in incident velocity increase the number of fragments found at the first peak around $120 \mu \mathrm{m}$, while decreasing the number of intact particles near the original particle size of $363 \mu \mathrm{m}$. Further runs of the numerical model indicated that the threshold velocity below which no particles fracture at $30^{\circ}$ incidence was $\sim 10 \mathrm{~m} / \mathrm{s}$. As will be discussed further in Section 5.4.5 and 5.4.6, particle fracture correlates with the kinetic energy transferred to the target in a direction perpendicular to the surface. Therefore, using this threshold fracture velocity, the corresponding magnitude of threshold kinetic energy transferred to the particle in perpendicular direction to surface could also be estimated and utilized to calculate the minimum velocity required for fracture at any other impact angle. For example, the threshold incident velocity for fracture of grit 60 particles at $60^{\circ}$ estimated in this manner is $5.8 \mathrm{~m} / \mathrm{s}$. Numerical simulations illustrated that, as expected, the minimum incident velocity for particle fracture at $60^{\circ}$ was lower than that of $30^{\circ}$ and was around $6 \mathrm{~m} / \mathrm{s}$. 


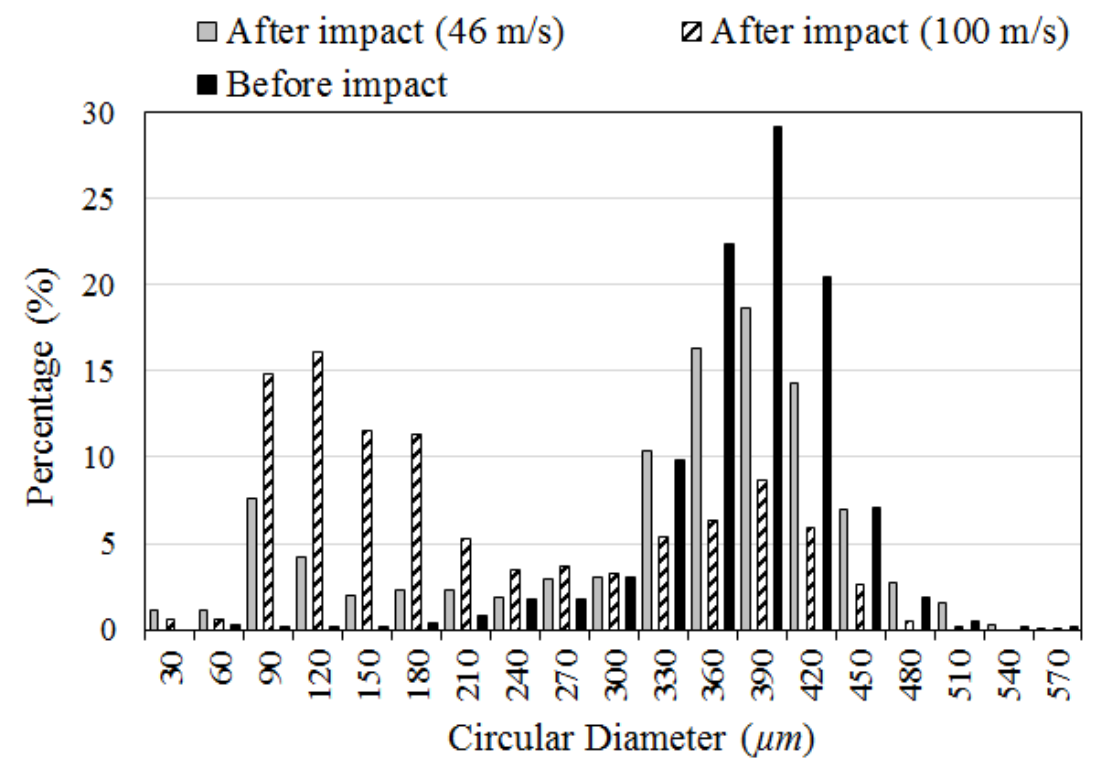

(a)

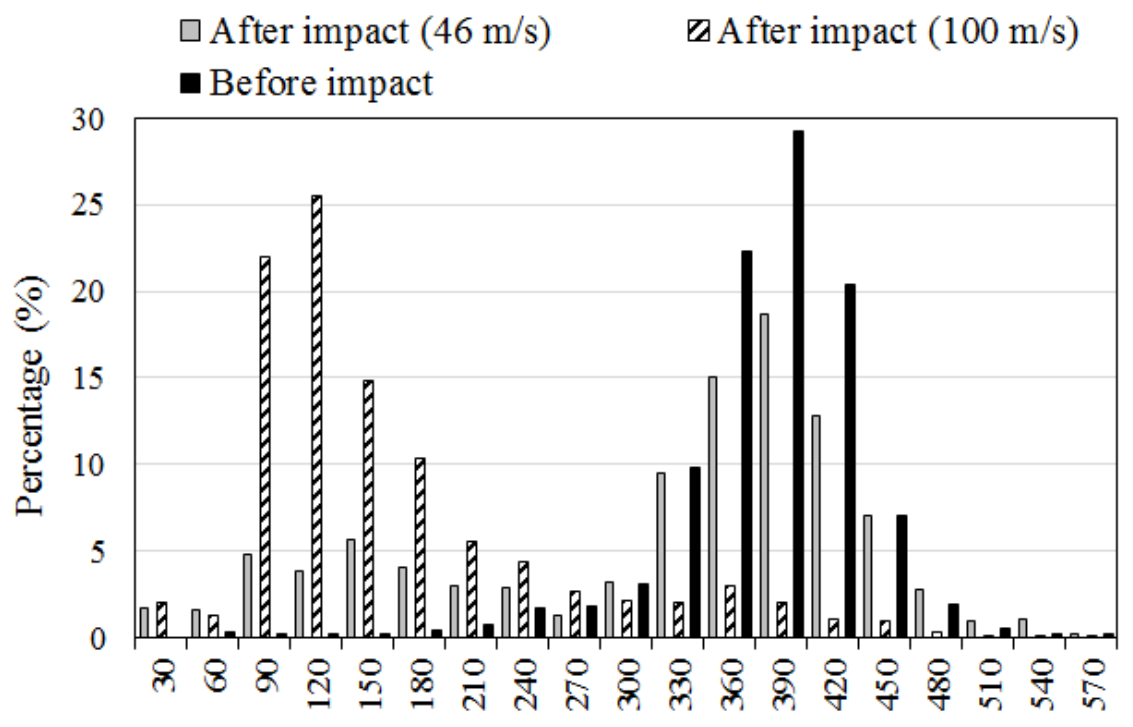

Circular Diameter $(\mu m)$

(b)

Figure 5.5 Measured size distributions of grit 60 particles before (average $=363 \mu \mathrm{m}$ ), and after impact for: (a) $30^{\circ}$ impact angle at $46 \mathrm{~m} / \mathrm{s}($ average $=323 \mu \mathrm{m})$ and $100 \mathrm{~m} / \mathrm{s}($ average $=211 \mu \mathrm{m})$;

(b) $60^{\circ}$ impact angle at $46 \mathrm{~m} / \mathrm{s}$ (average $\left.=309 \mu \mathrm{m}\right)$ and $100 \mathrm{~m} / \mathrm{s}($ average $=156 \mu \mathrm{m})$.

To better understand these trends, the model-simulated impact of a typical grit 60 particle at $30^{\circ}$ at $46 \mathrm{~m} / \mathrm{s}$ and $100 \mathrm{~m} / \mathrm{s}$ is shown in Fig. 5.6. Consistent with Figs. 5.5, the higher velocity particle resulted in a higher number of smaller fragments. 


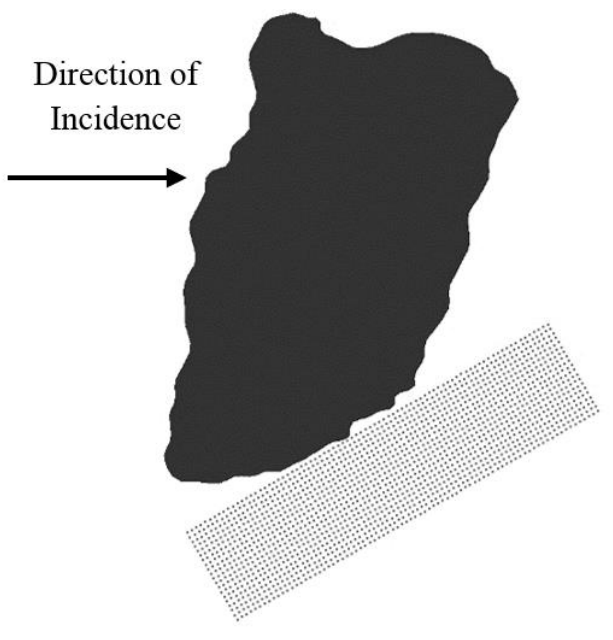

(a)

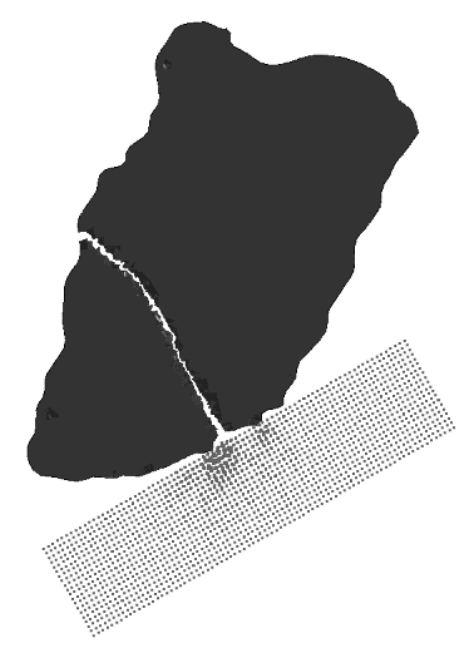

(b)

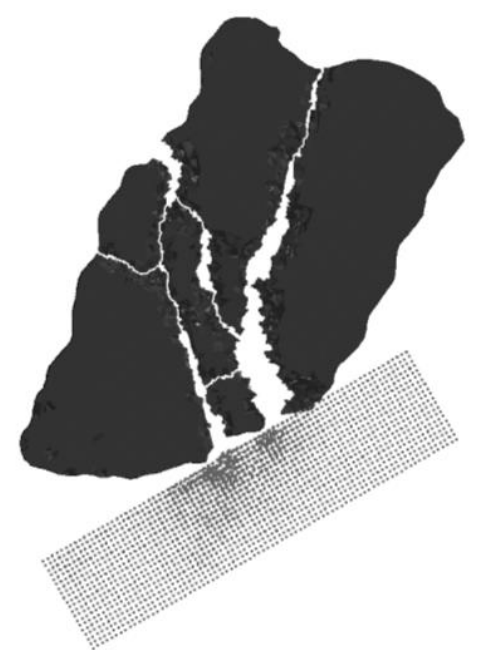

(c)

Figure 5.6 Typical grit 60 particle at $30^{\circ}$ impact angle (a) before impact (b) $7.5 \mu \mathrm{s}$ after impact at $46 \mathrm{~m} / \mathrm{s}$ (c) $7.5 \mu \mathrm{s}$ after impact at $100.3 \mathrm{~m} / \mathrm{s}$.

Figure 5.7 shows that the roundness of the grit 60 particles changed very little after impact under all conditions. Sparks and Hutchings [51] hypothesized that recycling of abrasives can result in progressive fracture and consequently changes in particle sharpness. The present results indicate that, while the particles did indeed become slightly sharper, the effect was not very strong after one cycle of blasting. 


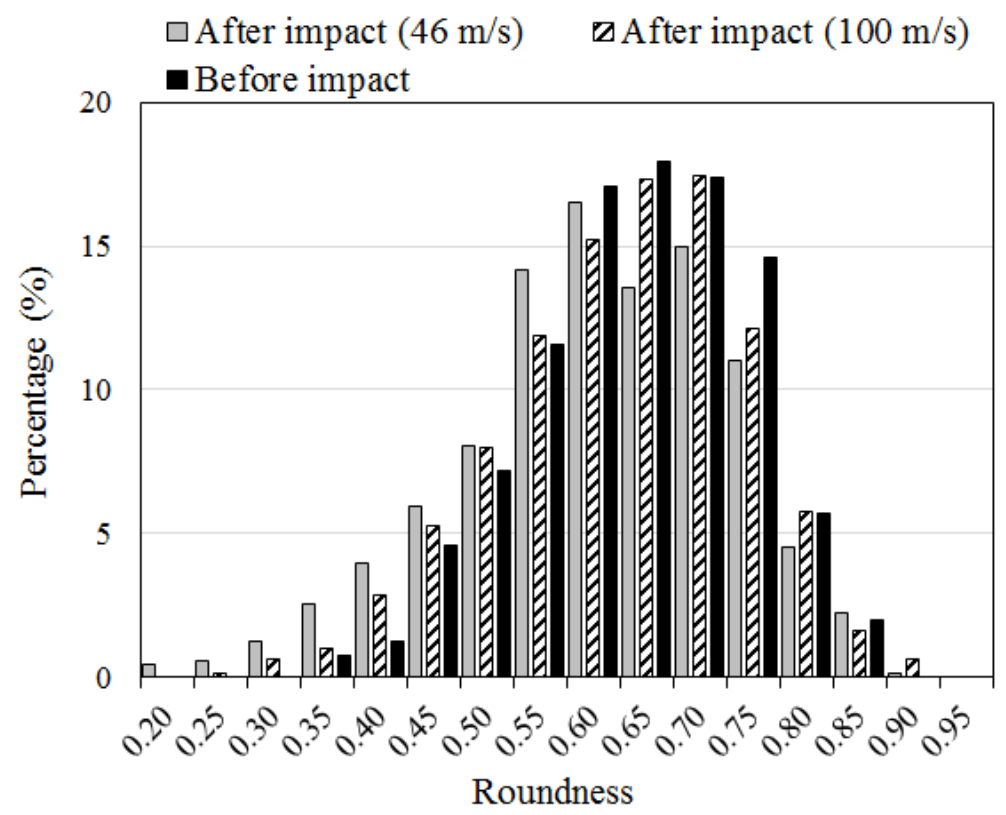

(a)

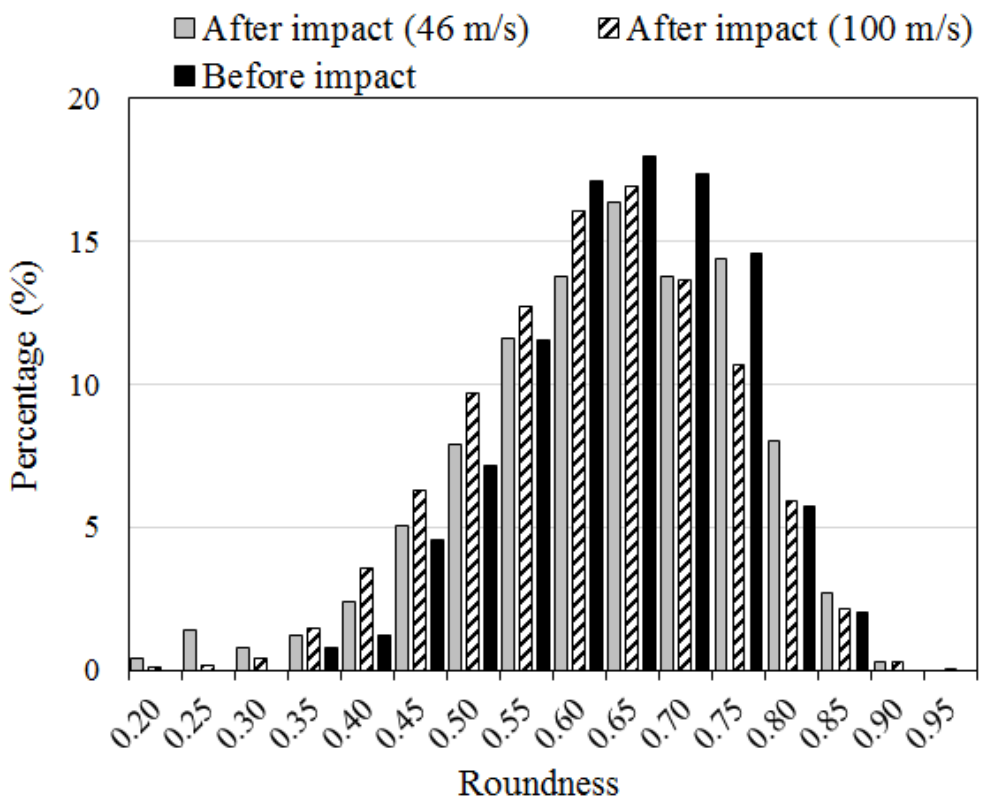

(b)

Figure 5.7 Measured roundness distributions of grit 60 particles before (average $=0.6$ ), and after impact for: (a) $30^{\circ}$ impact angle at $46 \mathrm{~m} / \mathrm{s}$ (average $=0.58$ ) and $100 \mathrm{~m} / \mathrm{s}$ (average $=0.6$ ); (b) $60^{\circ}$ impact angle at $46 \mathrm{~m} / \mathrm{s}$ (average $=0.6)$ and $100 \mathrm{~m} / \mathrm{s}($ average $=0.59)$.

Figure 5.8 shows the measured rebound angles for grit 60 particles after impact at incident velocities of $46 \mathrm{~m} / \mathrm{s}$ and $100 \mathrm{~m} / \mathrm{s}$, for incident angles of $30^{\circ}$ and $60^{\circ}$. For both impact angles, the 
average rebound angles were virtually unaffected by changes in velocity; however, for both impact angles, the range of rebound angles was wider at the higher velocity than the lower. The measured range of rebound angles in the present study was found to be wider than the range of 0.8-1.2 times the incident angle, measured by Slikkerveer and in't Veld for the impact of $23 \mu \mathrm{m}$ alumina particles at $200 \mathrm{~m} / \mathrm{s}$ on a soda-lime glass target [135].

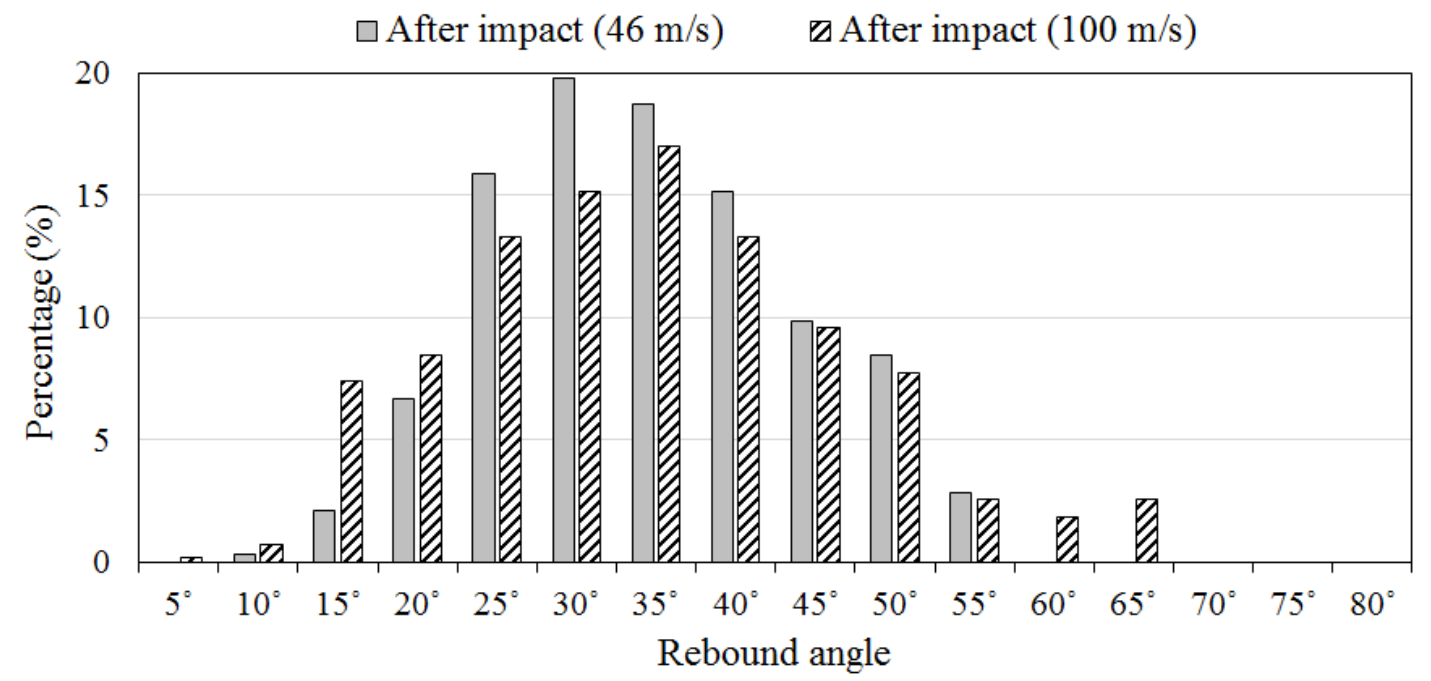

(a)

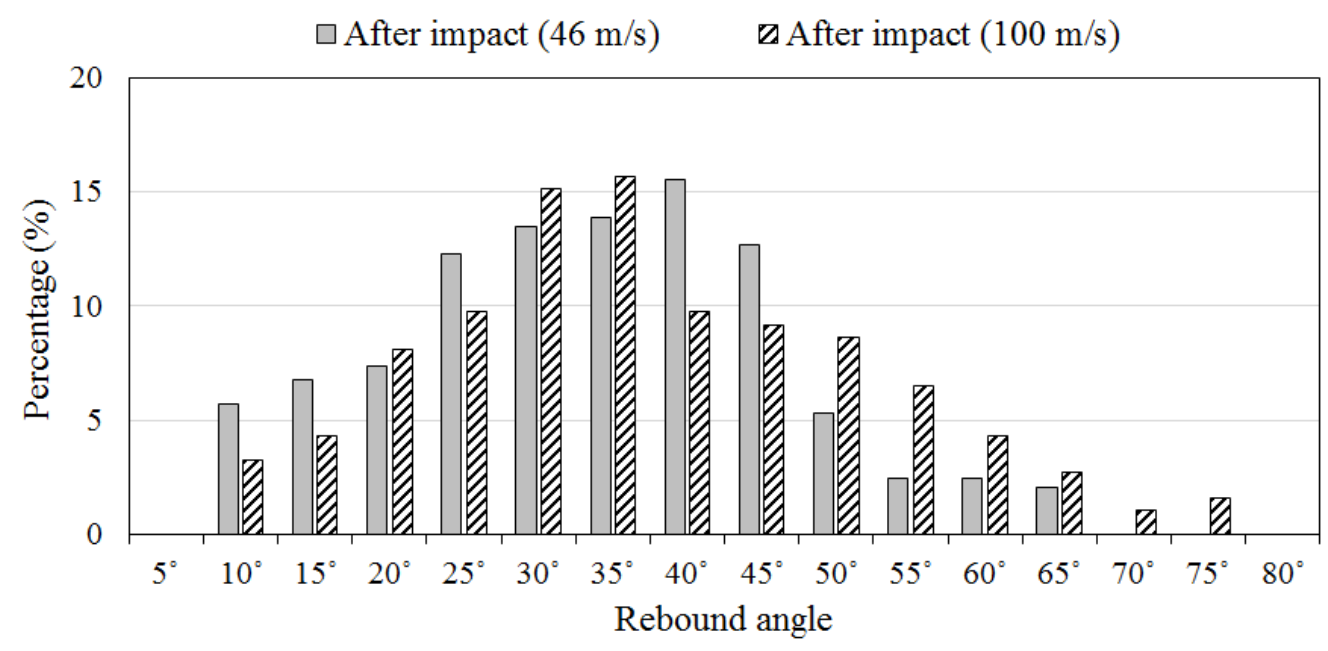

(b)

Figure 5.8 Measured rebound angle distribution of grit 60 particles after impact at 46 and 100.3 $\mathrm{m} / \mathrm{s}$ for (a) $30^{\circ}$ impact angle (averages: $27^{\circ}$ for $46 \mathrm{~m} / \mathrm{s}$ and $100 \mathrm{~m} / \mathrm{s}$ ) (b) $60^{\circ}$ impact angle (averages: $29.5^{\circ}$ and $31^{\circ}$ for $46 \mathrm{~m} / \mathrm{s}$ and $100 \mathrm{~m} / \mathrm{s}$, respectively). 
Since the total mass of abrasive remains constant before and after impact, the ratio of the average rebound to incident velocity (velocity ratio) can be used to evaluate the kinetic energy lost during impact, if rotational kinetic energy, usually small compared to linear [29,30], is neglected. The distributions of the velocity ratio for grit 60 particles at a $30^{\circ}$ and $60^{\circ}$ impact angle and for incident velocities of 46 and $100 \mathrm{~m} / \mathrm{s}$ are shown in Fig. 5.9. The ranges of the present study are in approximate agreement with the 0.2-0.5 range reported by Slikkerveer and in't Veld [135], even though their particle size and material, as well as target, was different than in the present study. The average velocity ratios at $30^{\circ}$ or $60^{\circ}$ (Fig. 5.9) did not change much with velocity. Since it was already demonstrated that fragmentation (and therefore energy consumed to fracture) increased with incident velocity, this implies that a higher percentage of initial incident energy was used in damaging the surface in the case of lower incident velocity.

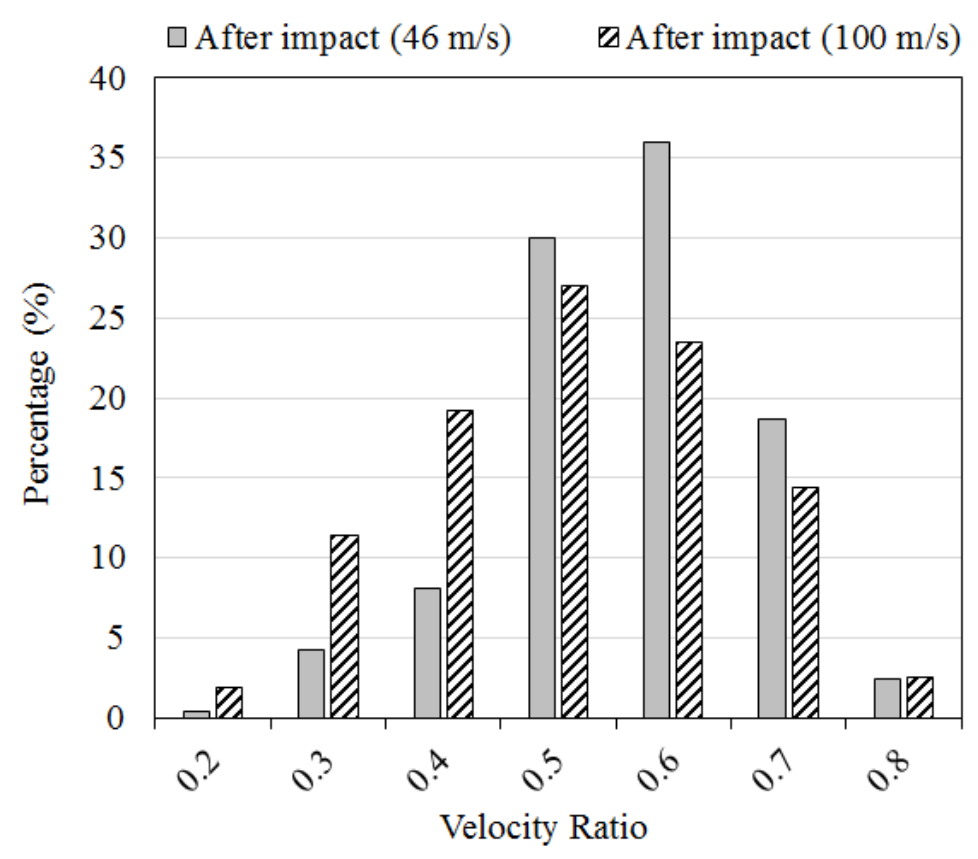

(a) 


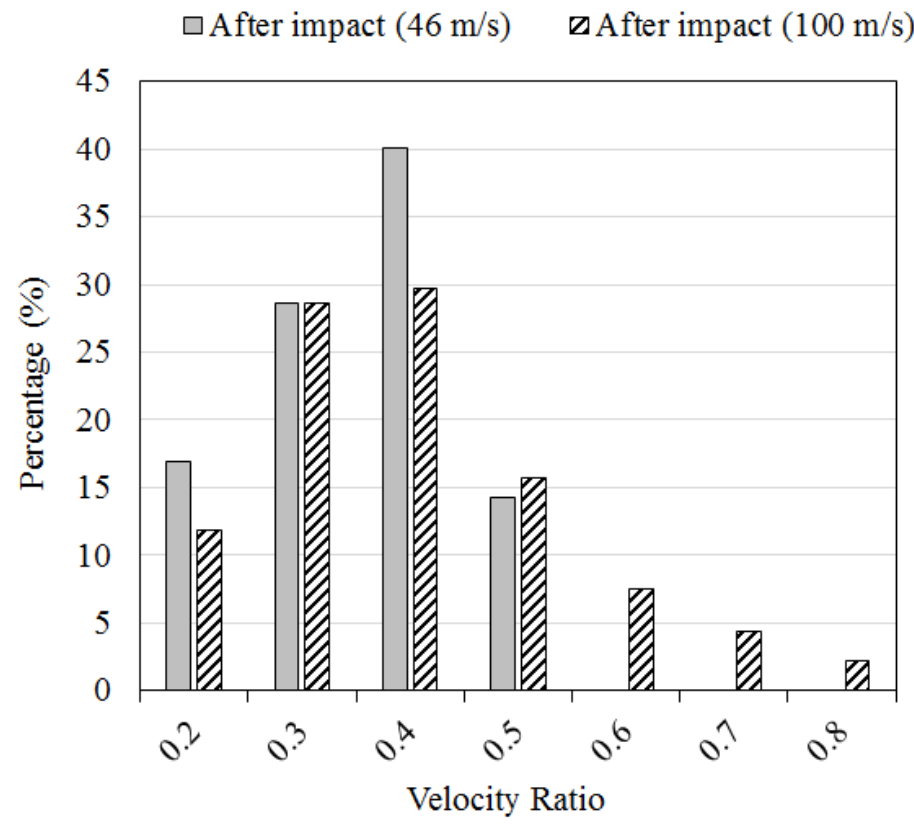

(b)

Figure 5.9 Measured velocity ratio distribution for grit 60 particles at $46 \mathrm{~m} / \mathrm{s}$ and $100 \mathrm{~m} / \mathrm{s}$ incident velocity at (a) $30^{\circ}$ impact angle (average: 0.51 and 0.48 for 46 and $100 \mathrm{~m} / \mathrm{s}$, respectively) (b) $60^{\circ}$ impact angle (average: 0.29 and 0.34 for 46 and $100 \mathrm{~m} / \mathrm{s}$, respectively).

\subsubsection{Influence of impact angle}

Comparisons of Figures 5.5a and 5.5b can be used to assess the influence of impact angle on fragmentation. At the relatively low velocity of $46 \mathrm{~m} / \mathrm{s}$, the dependence on impact angle was weak, i.e., the average circular diameters after blasting at $30^{\circ}$ and $60^{\circ}$ impact angle differed by $<5 \%$. However, at the higher $100 \mathrm{~m} / \mathrm{s}$ incident velocity, the dependence on angle was stronger, i.e. the average particle size after blasting differed by $\sim 36 \%$. These finding agree with those of Salman et al. [49] who reported that the number of fractured particles at a typical velocity decreases at lower impact angles. Even though the propensity to fracture was affected by the process parameters, Fig. 5.7 again shows that the average shape of the particles after impact did not change much regardless of impact angle and velocity, remaining close to that of particles before blasting.

Figure 5.9 shows that the average velocity ratio decreased with increasing impact angle (0.51 and 0.29 , respectively, for $30^{\circ}$ and $60^{\circ}$ at $46 \mathrm{~m} / \mathrm{s}$, and 0.48 and 0.34 , respectively for $30^{\circ}$ and $60^{\circ}$ at $100 \mathrm{~m} / \mathrm{s}$ ). This implies that the kinetic energy content of the rebounding particles after impact at a given velocity and $30^{\circ}$ was 2-3.1 times higher than at $60^{\circ}$. 
One of the reasons for the observed reduction in the kinetic energy content at higher impact angles is the expected dependencies of particle fracture and surface damage on energy transfers perpendicular to the surface, discussed further in Section 5.4.6. In other words, at higher impact angles, a greater percentage of initial kinetic energy was transferred perpendicular to the target, and consumed in damaging the surface and in large scale fracture of the particles. At shallower impact angles, most of the energy transfers occur parallel to the surface, resulting in a small amount of kinetic energy converted to rotational energy, and as discussed above, only small scale fracture occurring at the particle tips.

To better understand this, the model-simulated impact of a typical grit 60 particle at 100 $\mathrm{m} / \mathrm{s}$ incident velocity at $30^{\circ}$ and $60^{\circ}$ impact angle is shown in Fig. 5.10. As expected, a higher impact angle caused a greater extent of particle fracture and target damage.

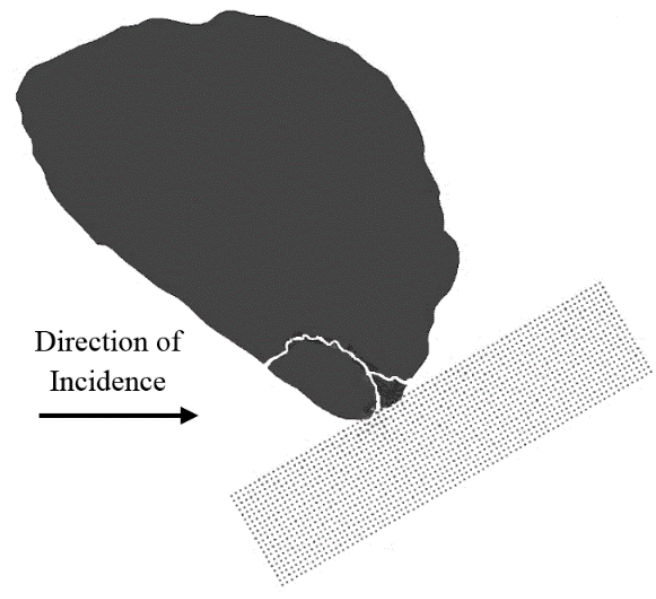

(a)

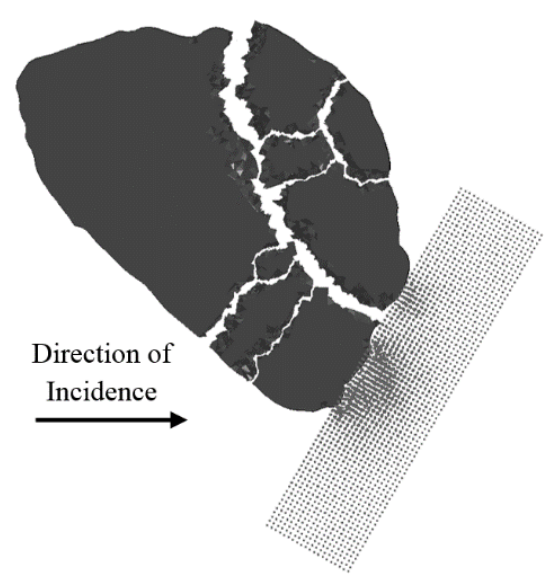

(b)

Figure 5.10 Typical grit 60 particle at $100 \mathrm{~m} / \mathrm{s}$ incident velocity (a) $7.5 \mu \mathrm{s}$ after impact at $30^{\circ}$ impact angle (b) $7.5 \mu \mathrm{s}$ after impact at $60^{\circ}$ impact angle.

Figure 5.8 shows that, as expected, the average rebound angle increased with increasing incident angle for both considered velocities. As illustrated more directly for the case of $46 \mathrm{~m} / \mathrm{s}$ in Fig. 5.11, the range of rebound angles also increased with increasing incident angle at both velocities. For example, at $46 \mathrm{~m} / \mathrm{s}$ and $30^{\circ}$, the rebound angle range was $5^{\circ}-49^{\circ}$, compared to the $1^{\circ}-61^{\circ}$ range at $60^{\circ}$. 


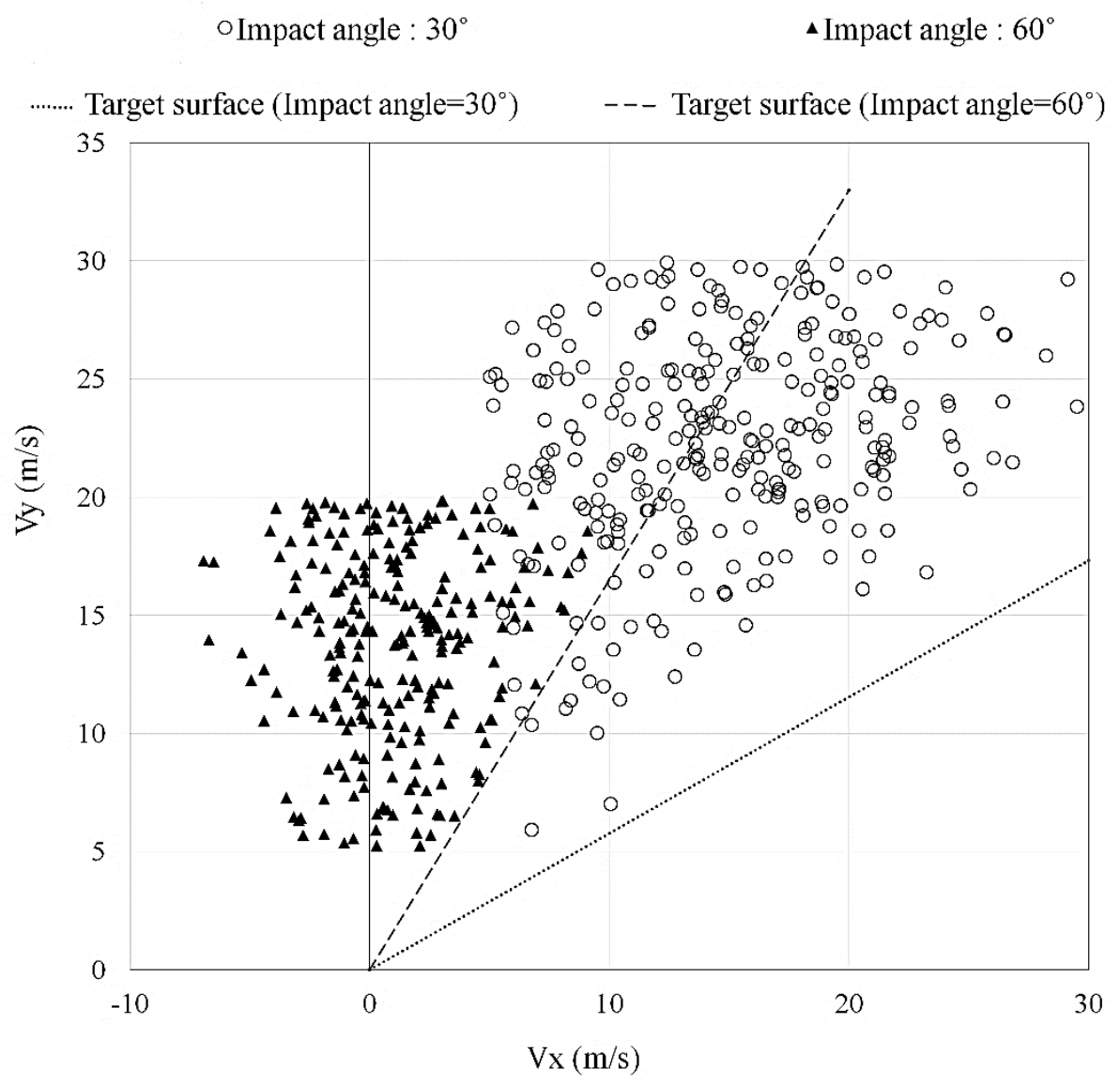

Figure 5.11 Scatter of $V x$ - $V y$ components of rebound velocity for grit 60 particles at $46 \mathrm{~m} / \mathrm{s}$ incident velocity and $30^{\circ}$ and $60^{\circ}$ impact angle. Lines indicate position of target surface.

\subsubsection{Influence of particle size}

Figure 5.12 shows that the numerical model was able to quite accurately predict particle fragmentation at the smaller grit 120 particle size. The average measured and predicted circular diameter after blasting at a $60^{\circ}$ impact angle and $100 \mathrm{~m} / \mathrm{s}$ differed by only $3.2 \%$, and the predicted and measured distributions were also very similar. The ratio of the average circular diameter of the particles before and after impact under these conditions was 1.62 for the grit 120 particles, which is lower than the 2.33 for grit 60 abrasives in Fig. 5.5b.

Analysis of the numerical results indicated that only $33 \%$ of the smaller grit 120 particles fractured compared to 53\% reported in Chapter 4 for the larger grit 60 particles at the same velocity and angle. Thus, the present model and experiments indicate that at a given velocity, larger particles are more prone to fragmentation, in agreement with the findings of Date and Malkin [166], Salman et al. [49], Akbarzadeh et al. [50] and Tilly and Sage [48]. 


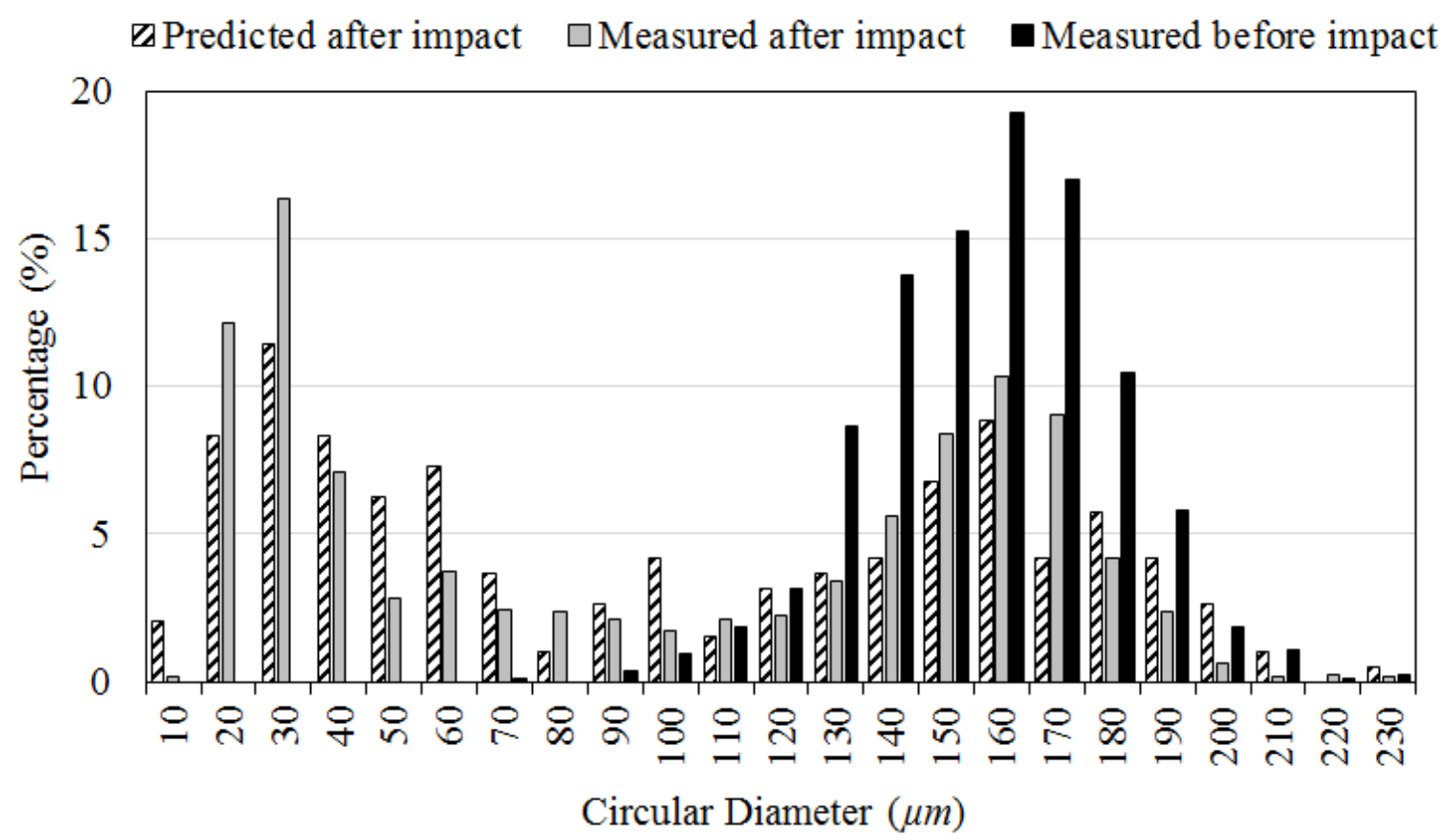

Figure 5.12 Predicted (average $=97 \mu \mathrm{m})$ and measured (average $=94 \mu \mathrm{m})$ size distributions of grit 120 particles after impact at $100 \mathrm{~m} / \mathrm{s}$ and a $60^{\circ}$ impact angle, compared to before impact (average $=152 \mu \mathrm{m})$.

The measured and predicted roundness of 120 particles after impact are shown in Fig. 5.13 with identical average values of 0.57 that differed only slightly from the roundness before blasting (0.58). Comparison of this roundness with that of grit 60 abrasives under the same test conditions (average $=0.59$, Fig. 5.7) shows that the abrasive size had only a small effect on the roundness of fragments. 


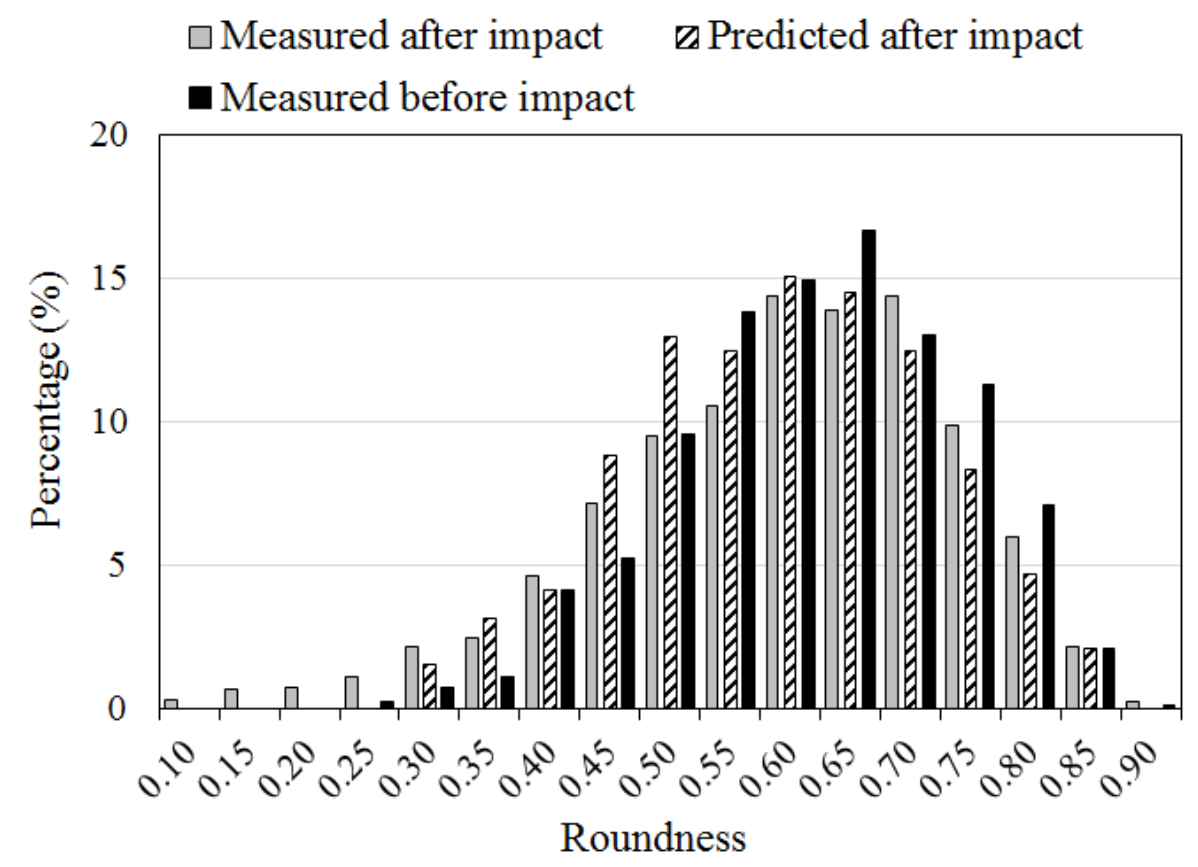

Figure 5.13 Predicted (average $=0.57$ ) and measured (average $=0.57$ ) roundness distributions of grit 120 particles after impact at $100 \mathrm{~m} / \mathrm{s}$ and a $60^{\circ}$ impact angle, compared to before impact (average $=0.58)$.

The influence of particle size on the extent of fracture at a given incident velocity is illustrated in Fig. 5.14 where typical abrasive particles were modeled with identical geometry but different size (grit 120 and 60) under the same impact conditions. In both cases, there was more fragmentation for the larger particle due to the higher kinetic energy content. In the first case (Figs 14a and b) the larger particle fractured, while there was no fracture for the smaller particle. The second case (Fig. 5.14c and 5.14d) show that the larger grit 60 abrasive particle fractured into larger number of fragments than the smaller grit 120. These trends of the dependence of fragmentation of particle size and kinetic energy are confirmed in Fig.5.15, which shows SEM images of the particles before and after impact at the same condition. 


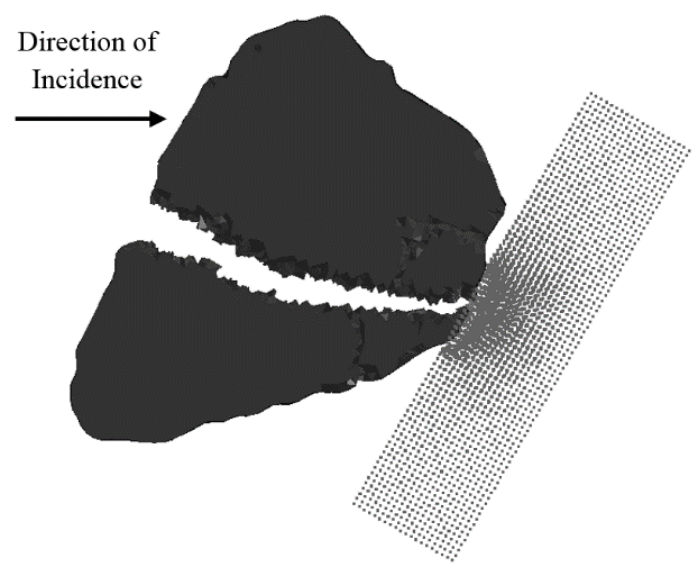

(a)

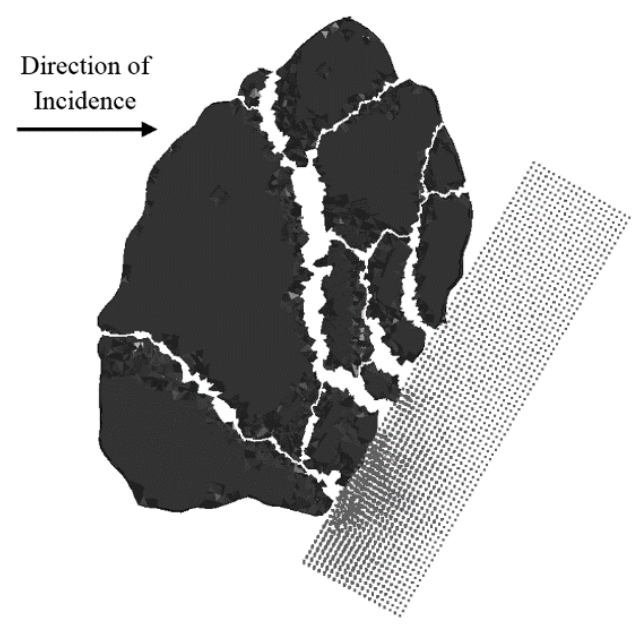

(c)

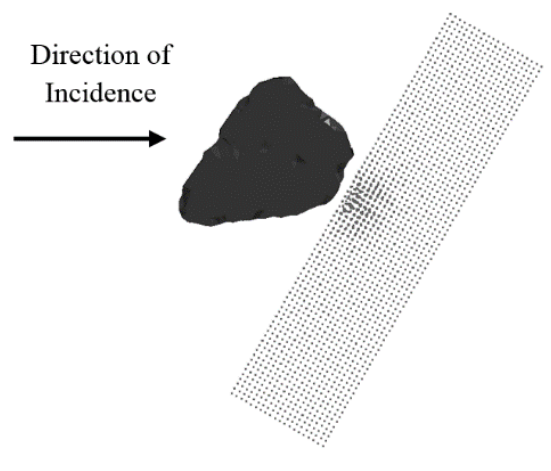

(b)

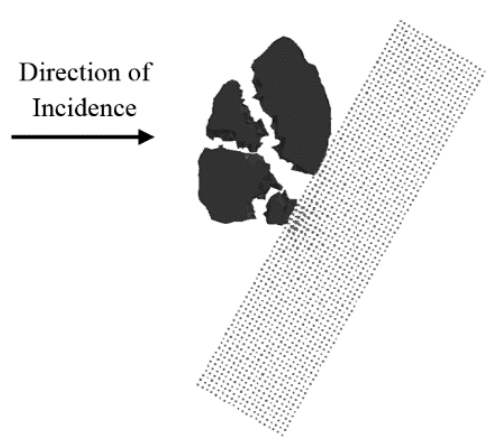

(d)

Figure 5.14 Comparison between the fracture of typical modeled particles of different sizes at $100 \mathrm{~m} / \mathrm{s}$ incident velocity $60^{\circ}$ impact angle (a) particle \#1, grit 60 ; (b) particle \#1, grit 120 ; (c) particle \#2, grit 60 (d) particle \#2, grit 120. 


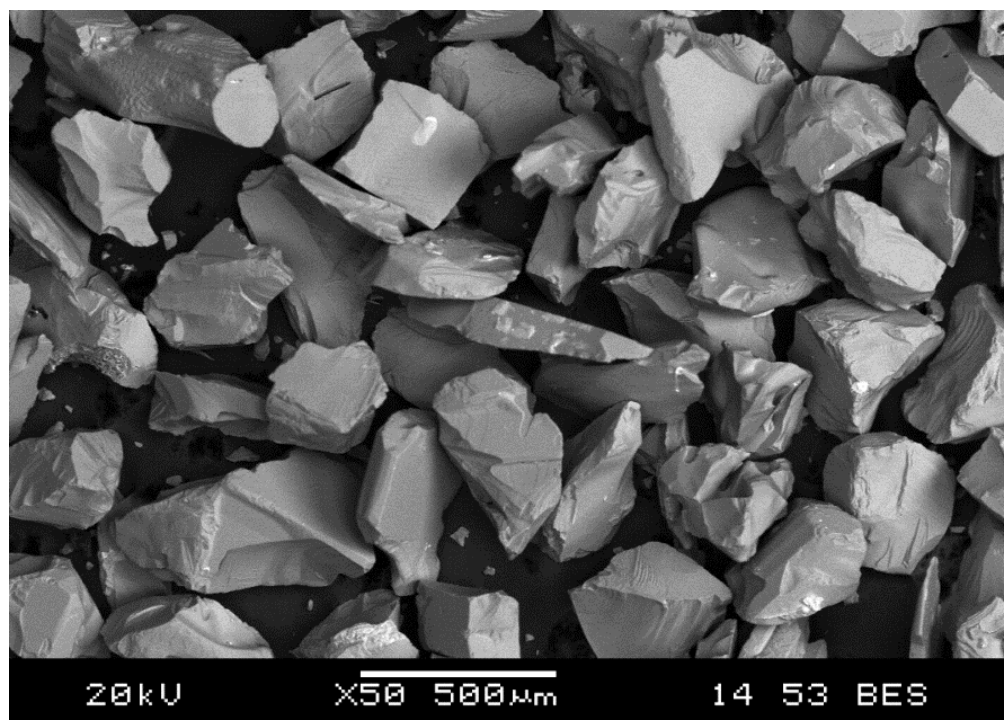

(a)

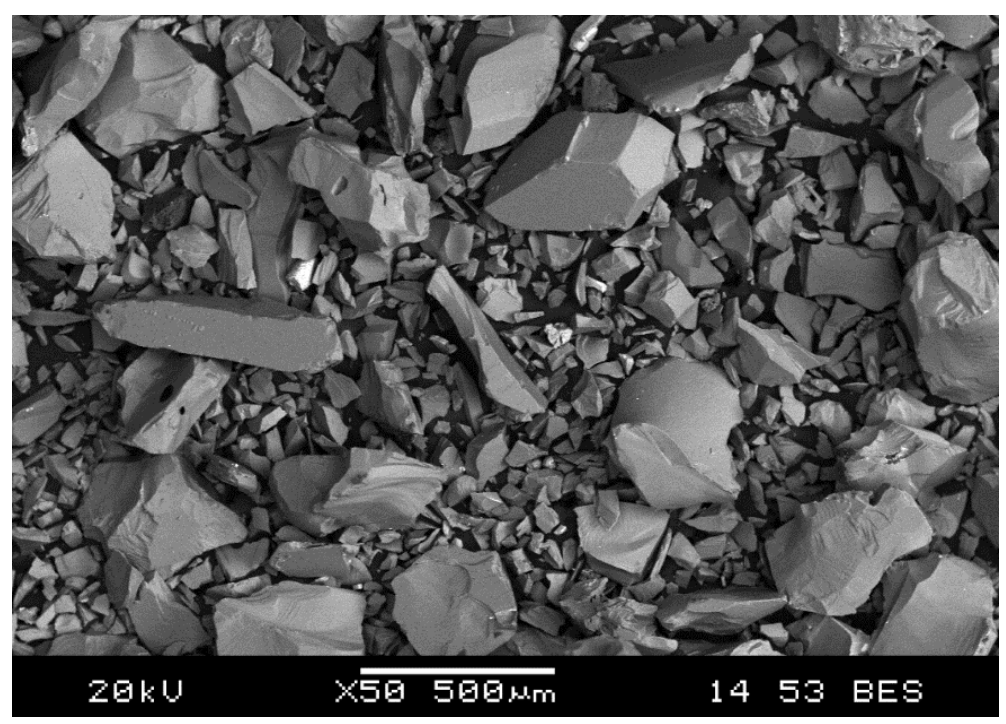

(b) 


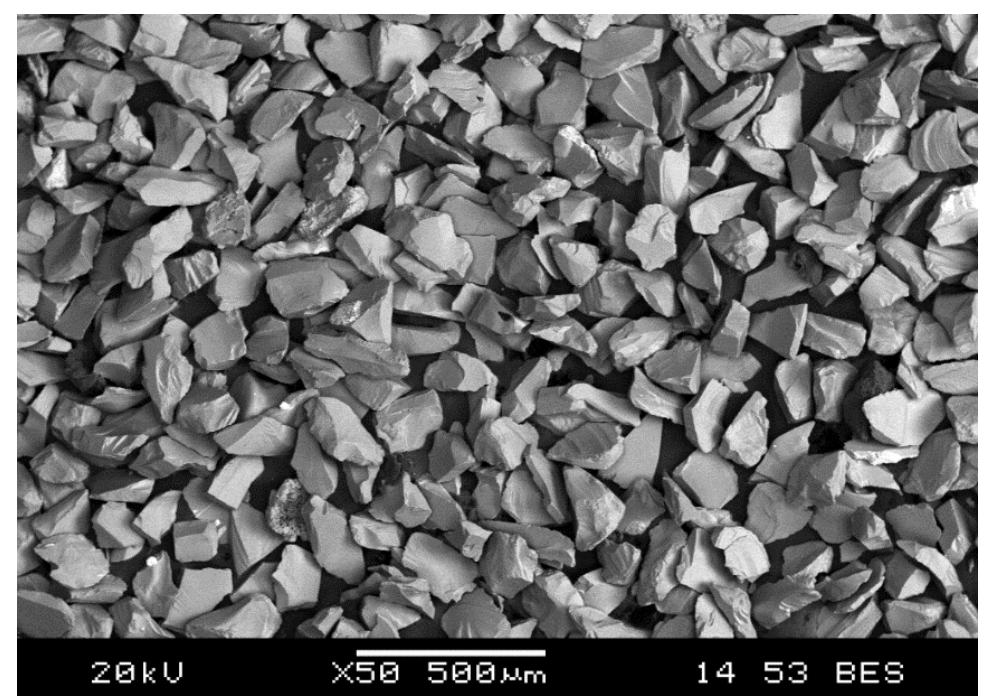

(c)

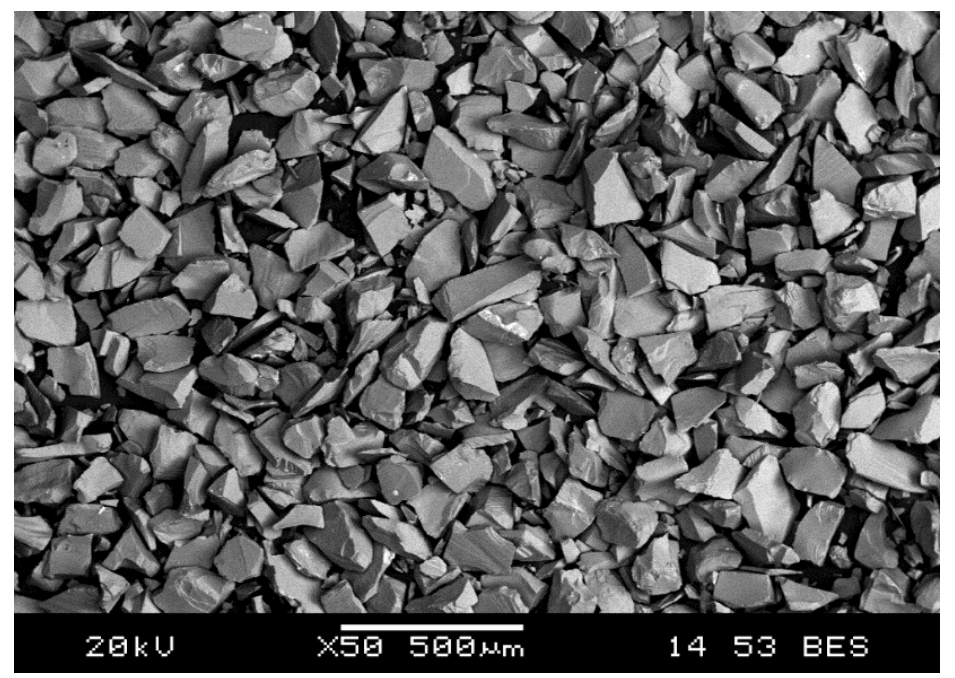

(d)

Figure 5.15 SEM images of particles before and after impact at $100 \mathrm{~m} / \mathrm{s}$ and $60^{\circ} \mathrm{impact}$ angle for (a) grit 60 before impact (b) grit 60 after impact (c) grit 120 before impact (d) grit 120 after impact.

The $X$ and $Y$ components of measured and predicted rebound velocity for grit 60 and 120 abrasives at $60^{\circ}$ impact angle at $100 \mathrm{~m} / \mathrm{s}$ are shown in Fig. 5.16. As with other cases, the model and experiments are in good agreement. 


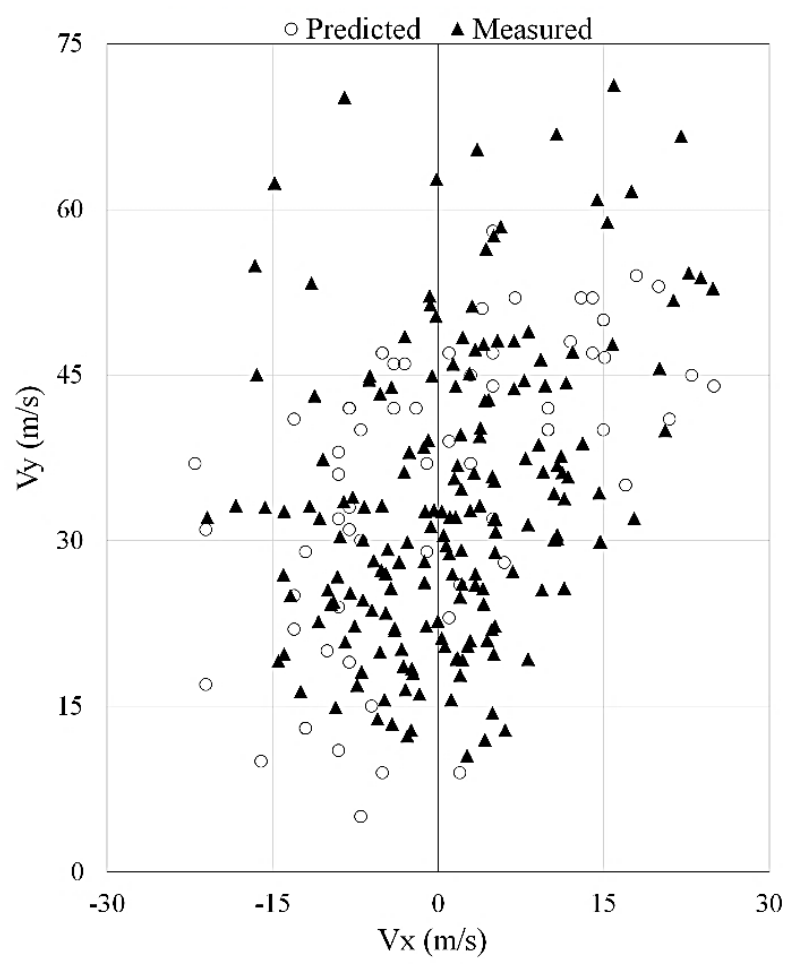

(a)

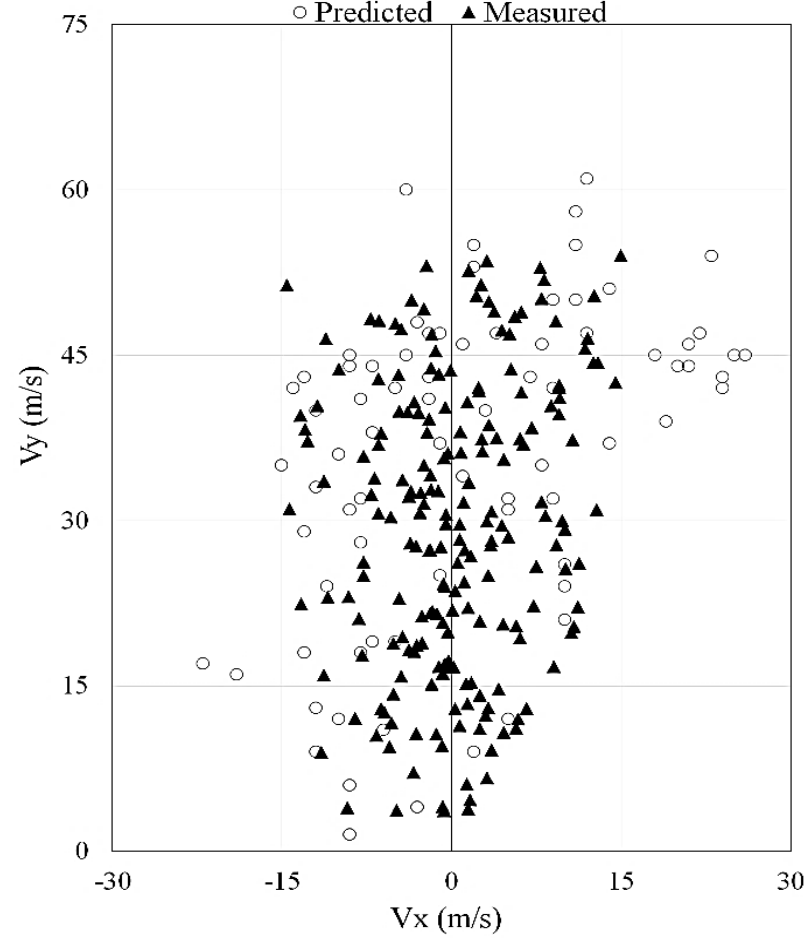

(b)

Figure 5.16 Distribution of measured and predicted values of $V x$ - $V y$ on rebound at $100 \mathrm{~m} / \mathrm{s}$ incident velocity and $60^{\circ}$ impact angle (a) for grit 60 and (b) for grit 120 abrasives.

The distribution of the velocity ratio for grit 60 and grit 120 abrasive particles after impact at $100 \mathrm{~m} / \mathrm{s}$ and $60^{\circ}$ are plotted in Fig. 5.17. The average measured velocity ratios for grit 60 and 120 particles were approximately 0.34 and 0.30 , which corresponds to $88 \%$ and $91 \%$ energy loss, respectively. Thus, at the same incident velocity and angle, the rebound velocities of the larger grit 60 particles after impact and fracture were slightly larger than that of the smaller grit 120 particles. 


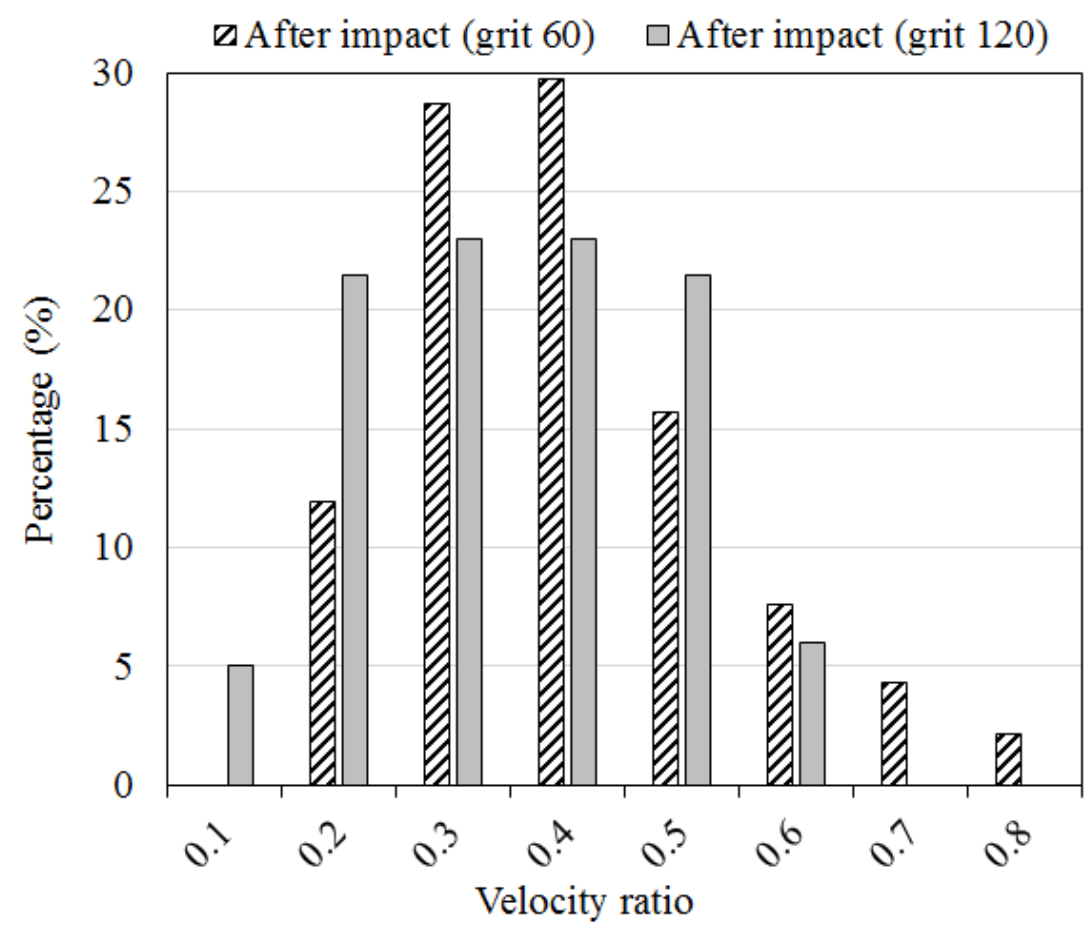

Figure 5.17 Distribution of the measured velocity ratio for grit 120 and 60 particles at $100 \mathrm{~m} / \mathrm{s}$ incident velocity and $60^{\circ}$ impact angle.

The range of measured rebound angle for grit 60 and grit 120 abrasives at $60^{\circ}$ incident angle is $1^{\circ}-67^{\circ}\left(\right.$ average $\left.=29.5^{\circ}\right)$ and $1.7^{\circ}-97^{\circ}\left(\right.$ average $\left.=30.5^{\circ}\right)$, respectively; and the predicted range of rebound velocity for grit 60 and grit 120 particles after impact under same condition is $1^{\circ}-72^{\circ}$ (average $=38.5^{\circ}$ ) and $2.5^{\circ}-111.5^{\circ}\left(\right.$ average $\left.=38^{\circ}\right)$, respectively. This comparison shows an acceptable consistency between the numerical predictions and experimental measurements. Moreover, comparison between the rebound angle of grit 60 and grit 120 abrasives after impact (Fig. 5.18) showed that at the same impact angle and incident velocity, the larger particles rebound at a narrower range of rebound angles, although the average values are fairly close. 


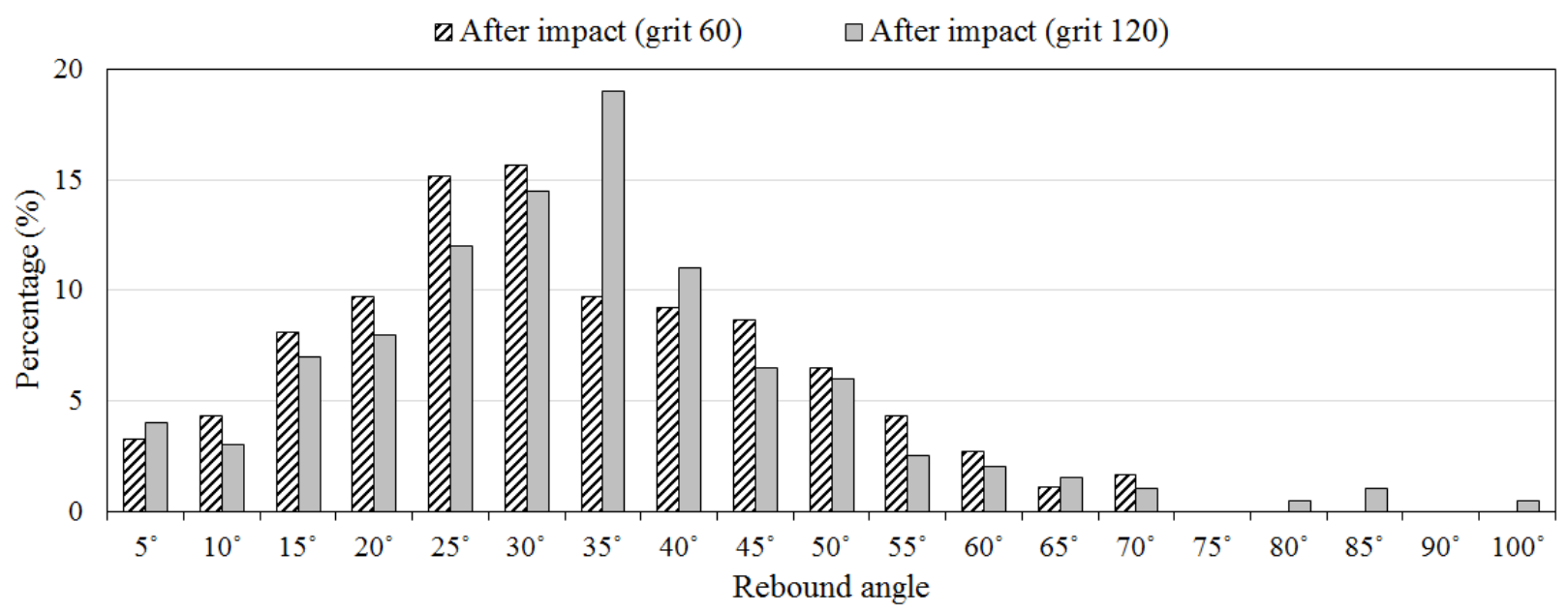

Figure 5.18 Distribution of the measured rebound angle for grit 60 (average $31^{\circ}$ ) and grit 120 (average $30.5^{\circ}$ ) particles at $100 \mathrm{~m} / \mathrm{s}$ incident velocity and $60^{\circ}$ impact angle.

As discussed in Section 5.1, investigators have attributed the tendency for larger particles to fracture at the same incident velocity to both the higher kinetic energy, and the apparent lower strength of the larger abrasives. Numerical models of particle fracture for 100 grit 60 and grit 120 particles at identical average incident kinetic energies of $0.1574 \mathrm{~mJ}$ at $60^{\circ}$ incidence were constructed in order to separate these two factors. The results for grit 120 abrasives at $373 \mathrm{~m} / \mathrm{s}$ and grit 60 particles at $100 \mathrm{~m} / \mathrm{s}$ (so as to produce the same average kinetic energy) indicated that all of the grit 120 particles fractured, compared to only $53 \%$ of the grit 60 abrasives. Moreover, numerical results showed that $33 \%$ of grit 120 particles fractured at $100 \mathrm{~m} / \mathrm{s}$, which is greater than the $6 \%$ found for larger grit 60 particles at $27 \mathrm{~m} / \mathrm{s}$ (average incident kinetic energy of $0.0115 \mathrm{~mJ}$ in both cases).

As discussed above, the present model predicts that smaller particles show a lower resistance to fracture at the same incident kinetic energy. Simulation of the same particles shown in Fig. 5.14c and 5.14d, but now impacting at the same kinetic energy, shows that the principle strain is around $0.3 \%$ for the grit 60 particle (Fig. 5.19a), which is noticeably lower than the $\sim 1 \%$ generated for the same modeled grit 120 particle (Fig. 5.19b). This suggests that the smaller particles show a higher propensity for fracture at a given kinetic energy because the generated strain is more localized. Limitations in the utilized blasting apparatus unfortunately made it impossible to directly verify this prediction. Although it appears to be no data for fracture of multiple sizes of angular particles at the same kinetic energy in the literature, analysis of the data 
reported by [49] for fracture of $3.15 \mathrm{~mm} .5 .15 \mathrm{~mm}$ and $7.15 \mathrm{~mm}$ spherical alumina particles after impact at various velocities can be used indirectly to justify this prediction. For example, extrapolating the data from [49], for the $5.15 \mathrm{~mm}$ particle at $20 \mathrm{~m} / \mathrm{s}, \sim 60 \%$ of particles were reported to fracture, while at $42 \mathrm{~m} / \mathrm{s} 100 \%$ of $3.15 \mathrm{~mm}$ particles were reported to fracture. Since both of these particles have the same initial kinetic energy, i.e. $20 \mathrm{~m} / \mathrm{s}$ x $(0.00515 \mathrm{~m})^{3} \sim 42 \mathrm{~m} / \mathrm{s}$ $\mathrm{x}(0.00315 \mathrm{~m})^{3}$, the data indicates that the smaller particles were more likely to fracture at the same incident kinetic energy. Similarly, $30 \%$ of $7.15 \mathrm{~mm}$ particles were reported to fracture at $10 \mathrm{~m} / \mathrm{s}$, while for $3.15 \mathrm{~mm}$ particles at the same kinetic energy, i.e. at $34 \mathrm{~m} / \mathrm{s}, 100 \%$ of the particles fractured.

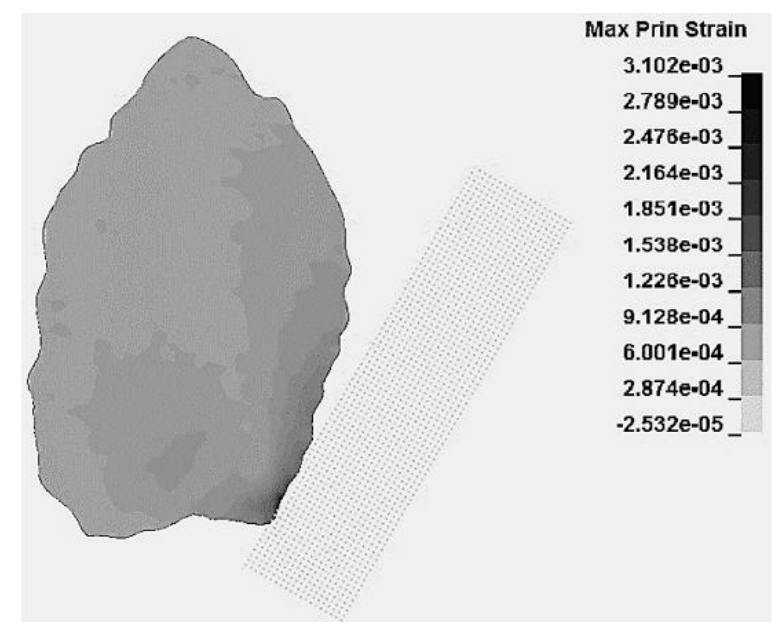

(a)

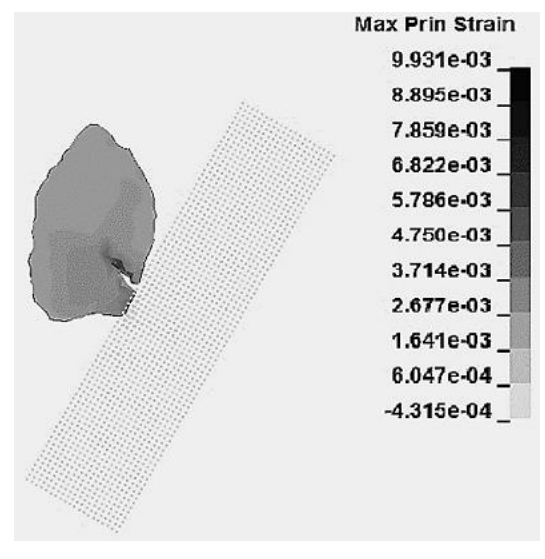

(b)

Figure 5.19 Contour plot of maximum principle strain for an impacting particle modeled in different sizes, after $t=0.25 \mu \mathrm{s}$ from impact at $60^{\circ}$ angle (a) particle \#2, grit $60,27 \mathrm{~m} / \mathrm{s}$ incident velocity ; (b) particle \#2, grit $120,100 \mathrm{~m} / \mathrm{s}$ incident velocity.

\subsubsection{Influence of kinetic energy transfers perpendicular to surface on particle fracture}

As shown in sections 5.4.3 and 5.4.4, the particle diameter after impact decreased with increasing incident velocity and angle of attack, indicating that energy transfers perpendicular to the surface played a large role on the propensity for fracture. As shown in Fig. 5.20, the average number of fragments per incident particle $\left(N_{f} / N_{i}\right)$, where $N_{f}$ and $N_{i}$ are the number of particles after and before impact, respectively) was found to correlate strongly in an almost linear fashion with the kinetic energy associated with incident normal velocity (the 'normal kinetic energy'). 
$N_{f} / N_{i}=\left(D_{i} / D_{f}\right)^{3}$ was calculated based on conservation of mass before and after impact, i.e.,.:

$N f * \rho\left(\frac{4}{3} \pi\right) *\left(\frac{D_{f}}{2}\right)^{3}=N i * \rho\left(\frac{4}{3} \pi\right) *\left(\frac{D_{i}}{2}\right)^{3}$

where $\rho, D_{i}$ and $D_{f}$ are the particle density, the average measured circular diameter of abrasives before and after impact, respectively.

The average threshold value of normal kinetic energy required for fracture of grit 60 abrasive was $\sim 4 \times 10^{-4} \mathrm{~mJ}$. This threshold can be achieved at different combinations of incident angle and velocity; i.e., $10 \mathrm{~m} / \mathrm{s}$ incident velocity at $30^{\circ}$ impact angle, or $\sim 6 \mathrm{~m} / \mathrm{s}$ incident velocity at $60^{\circ}$ as mentioned in section 5.4.3.

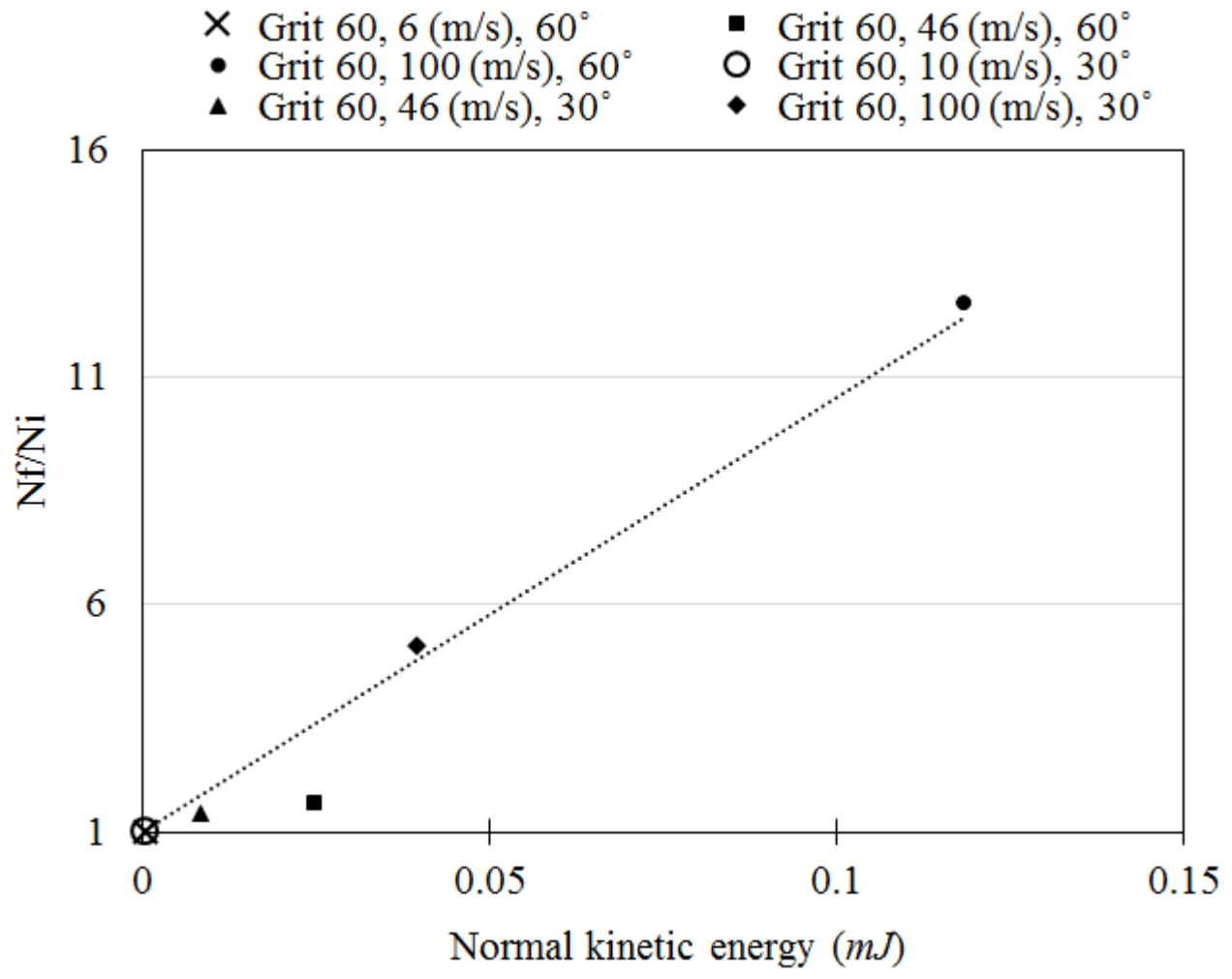

Figure 5.20 Dependence on number of particle fragments per launched particle on normal kinetic energy for grit $60 \mathrm{SiC}$ abrasives under different operating conditions. The dotted line is a least squares linear fit. 


\subsection{Summary}

Numerical modeling and shadowgraphy experiments were used to study the influence of process parameters on the fracture and rebound kinematics of abrasives upon impact. Further experimental evidence was presented to show that the numerical fracture model could be applied for variety of conditions; i.e., for a less severe impact condition and for much smaller particles than considered in Chapter 4. It was demonstrated that fundamental data provided by the model such as percentage of incident particles that fracture, could be used to better understand the process mechanics. Other principle findings can be summarized as follows:

i) The particle size after impact gives a good indication of the propensity for fracture since it can be correlated to the percentage of incident particles that fractured.

ii) Particles were more likely to fracture at high incident angle and velocity, or more fundamentally, under conditions that transferred higher kinetic energy to the particles in a direction normal to the target. A threshold normal kinetic energy for fracture of grit 60 $\mathrm{SiC}$ particles was identified.

iii) Utilizing a same incident velocity, larger particles showed a higher propensity for fracture than smaller ones. This was shown to be due to the fact that the associated incident kinetic energy was higher for larger particles.

iv) At the same incident kinetic energy, smaller particles were found to be more likely to fracture than large ones. This was found to be due to the more localized strain in the smaller particles.

v) Measurement of particle residuals after impact under various conditions illustrated that the average roundness did not change noticeably, however, a wider range of roundness was observed after impact than before.

vi) Numerical modeling and shadowgraphy experiments showed that, while using a same impact angle and particle size, variation of incident velocity did not change the velocity ratio and percentage of lost kinetic energy; however, the velocity ratio and remaining kinetic energy reversely depend on impact angle. 


\section{Summary and Conclusions}

\subsection{Summary}

Solid particle erosion is an important phenomenon with many constructive and destructive applications in various applications. Particle embedment and particle fracture are two important, but little studied, phenomena that occur during solid particle erosion. Chapter 2 presented measurements of, and a model for predicting, the distribution of instantaneous angular particle orientations in an abrasive air jet under conditions that are typical of solid particle erosion testing and abrasive jet machining applications. Chapter 3 utilized the results of Chapter 2 to develop and experimentally verify a SPH-FEM simulation with appropriate constitutive models and realistic angular particle geometries, capable of predicting the embedment of $\mathrm{SiC}$ abrasives in Aluminum 6061 -T6 target, and to assess the influence of different operating parameters on the propensity for particles embedment. Chapter 4 introduced a numerical model that utilized a $\mathrm{JH}$ material model, and EFG formulations to simulate the fracture of abrasives upon impact occurring in a typical solid particle erosion application. The models utilized a novel particle geometry generation scheme, and were verified by comparison with measurements made using double-pulsed laser shadowgraphy, which made it possible to capture the fracture of the particles at the instant of impingement. Chapter 5 used the models of Chapter 4 and further experiments to determine the influence of process parameters such as particle size, incident velocity, impact angle and kinetic energy on the extent of fracture, the fragment shape and size, and fragment rebound kinematics. A minimum velocity required for particle fracture at any angle of incidence was determined and linked to a minimum threshold kinetic energy incident perpendicular to the surface required for particle fracture.

\subsection{Conclusions}

The principle conclusions of the present dissertation can be summarized as follows:

(i) Although up to $\sim 30 \%$ of the particles were oriented with the jet axis at the nozzle exit, a statistically significant tendency was found for the likelihood of orientation to increase by up to 1.24 times at a distance of $40 \mathrm{~mm}$ from the jet exit. 
(ii) The model that considered rotation of angular particles in the calculation of the instantaneous drag force was found to predict the linear velocity and instantaneous orientation distribution of particles in flight with a good agreement with experiments.

(iii) Larger particles were more likely to orient themselves with the jet than smaller ones. There was also a tendency for high aspect ratio particles to align with the jet. However, the influence of pressure on particle orientation at a given standoff was found to be insignificant.

(iv) The friction coefficient between the SiC particles and the Al6061-T6 target was determined as 1.5 through calibration of the numerical model by comparing the predicted and measured embedment percentage from the numerical model for one particle size, standoff, and velocity at perpendicular incidence.

(v) Using this friction coefficient in the numerical models under a wide variety of conditions resulted in predictions of embedment that were at most 1.16 times higher than those measured.

(vi) The likelihood of embedment strongly depended on the particle orientation. Numerical simulations illustrated that particles that hit the target in orientations that were not aligned with the jet were found to not embed even when using large friction coefficients.

(vii) The models and experiments showed that, for the same shape and impact velocity, larger particles were more likely to embed. It was also shown that for a given particle size, increases in particle velocity enhanced the likelihood of particle embedment.

(viii) For the first time, double-pulsed laser shadowgraphy was utilized to capture the impact, fracture and fragmentation of grit $60 \mathrm{SiC}$ particles impacting an Al6061-T6 target at 46 and $100 \mathrm{~m} / \mathrm{s}$ and $30^{\circ}$ and $60^{\circ}$ impact angle. Analysis of the captured images and measurement of the particle fragments after impact revealed a marked change in the particle size distribution due to fragmentation upon impact. 
(ix) The incident and rebound velocity of the particles was measured using the shadowgraphic method. The average rebound velocity was typically $\sim 0.29-0.34$ times lower than the average incident velocity.

(x) For the first time, a coupled EFG and SPH technique was used to model the fracture of $\mathrm{SiC}$ particles upon impact on an aluminum alloy target. Numerical predictions and experimental measurements of particles geometrical characteristics after impact/fracture, were found to be in agreement; i.e., average size $(<3.2 \%$ difference), roundness $(<9.2 \%$ difference). The model also could predict the kinematics of the rebounding particle fragments with good accuracy.

(xi) Particles were more likely to fracture at high incident angle and velocity, or more fundamentally, under conditions that transferred higher kinetic energy to the particles in a direction normal to the target. A threshold normal kinetic energy for fracture of grit $60 \mathrm{SiC}$ particles was identified.

(xii) Utilizing a same incident velocity, larger particles showed a higher propensity for fracture than smaller ones. This was shown to be due to the fact that the associated incident kinetic energy was higher for larger particles. Nevertheless, at the same incident kinetic energy, smaller particles were found to be more likely to fracture than large ones. This was found to be due to the more localized strain in the smaller particles.

(xiii) Measurement of particle residuals after impact under various conditions illustrated that the average roundness did not change noticeably, however, a wider range of roundness was observed after impact than before.

(xiv) Numerical modeling and shadowgraphy experiments showed that, for a given impact angle and particle size, changes in incident velocity did not affect the velocity ratio and percentage of lost kinetic energy; however, the velocity ratio and remaining kinetic energy inversely depend on impact angle. 


\subsection{Practical implications}

The results of this research can be utilized to optimize industrial processes such as solid particle erosion testing, abrasive jet machining and blast stripping. For instance, considering that particle embedment is an unwanted phenomenon that can reduce the erosion rate, and with respect to the direct influence of particle size and standoff distance on particle orientation and embedment, the present research suggested that large standoff distances and large abrasive particles should be avoided. Moreover, it is also suggested that high impact angles and incident velocities that increase the particle embedding should not be used. The results with respect to the effect of friction on particle embedment and penetration depth suggest that utilizing sharp particles with smooth surfaces are also likely to reduce particle embedment whilst also increasing the erosion rate.

The fracture of abrasives during solid particle erosion processes can waste a portion of incident kinetic energy that might otherwise be used to cause surface erosion due to the primary impacts. For example, as mentioned in section 5.4.3, the velocity ratios of grit 60 particles at 100 and $46 \mathrm{~m} / \mathrm{s}$ incident velocity were almost similar, implying a similar level of consumed energy during impact. Given that the extent of particle fracture at $100 \mathrm{~m} / \mathrm{s}$ was noticeably higher, this suggests that a greater portion of the initial incident kinetic energy was consumed in erosion at the lower incident velocity. With respect to the influence of energy consumed for fracture, in reducing the erosion caused by the initial abrasive impacts, it can be concluded that smaller abrasives at shallower impact angles are more appropriate for maximizing erosion, and that high incident velocities which result in excessive particle fracture should to be avoided. Numerical predictions and experimental measurements of rebound angle and velocity can also be utilized to predict the propensity of particle collision in an erosion test. For example, a low rebound velocity can result in a higher likelihood of particle collision and thus, more wasted energy due to particle fracture. The presently developed methodology for predicting and measuring particle rebound velocity and angle can also be utilized to improve the surface evolution models while considering the secondary impact of particles in an abrasive jet machining application. 


\subsection{Contributions}

The novel contributions of the present study are:

(i) For the first time, the distributions of the instantaneous orientation angle of particles within abrasive jet were measured. The presented model is the first that allows prediction of orientation, and considers orientation in the prediction of linear velocity. It is also the first attempt to correlate embedment directly to actual particle orientation measurements within a jet.

(ii) The model presented in Chapter 3 is the first numerical model capable of predicting particle embedment into a ductile metal. It distinguishes itself from the only other existing model for embedment by Getu et al. [16] through its use of irregular and realistic particle geometries, the consideration of strain, strain rate hardening and thermal softening, and the use of a much larger range of particle sizes and impact velocities. In contrast, Getu et al.'s model utilized idealized rhomboid particle geometries impacting a perfectly plastic target [16].

(iii) A new methodology for determination of the friction coefficient for single abrasive grains in contact with the target was proposed in Chapter 3. The technique is based on calibration of the numerical model through a comparison of measured and predicted percentage of embedded particles.

(iv) The coupled EFG-SPH numerical model presented in Chapter 4 is the first simulation capable of predicting particle fracture for typical solid particle erosion applications. To provide geometrical input to this model, a novel technique was introduced to directly convert shadowgraphic images into realistic three-dimensional CAD representation of particles in their actual orientation while in flight.

(v) For the first time, images of the fracture of particles upon impact on a target were captured using double-pulsed laser shadowgraphy. The technique allowed the distributions of particle fragment size, roundness, velocity, and rebound angle to be measured for the first time.

(vi) For the first time, a numerical model was used to determine the minimum normal kinetic energy and corresponding value of threshold incident velocity required for the fracture of abrasive particles. A correlation between the proportion of launched 
particles that fractured and the average size of particle residuals after impact was established for the first time.

(vii) Previously, prevailing knowledge was that larger particles were more likely to fracture than smaller ones. Novel experiments and modeling of fracture at both constant kinetic energy and constant incident velocity allowed for a deeper insight into the mechanisms governing the dependence of fracture on particle size.

\subsection{Recommendations for future work}

The research presented in this dissertation could be extended to study the following other interesting aspects of solid particle erosion

(i) Modeling of particle embedment after previous embedment has occurred. The present embedment model used a virgin un-eroded target. In reality, a target will be rapidly subjected to embedment, and it is important to investigate how or whether previously embedded particles affect the embedment of subsequent particles. This could be accomplished by changing the target geometry in the model of Chapter 3 from a homogeneous aluminum alloy to a composite material containing previously embedded particles.

(ii) Simulation of particle embedment in multi-particle impact applications. In real AJM applications, multiple particles strike the surface simultaneously and a steady state level of embedment is reached. This multi-impact case could be modeled to gain insight about the cumulative effect of strain rate, strain hardening and temperature softening induced in the material by repeated impacts.

(iii) Analysis of radial wash of fragments on erosion rate. The present study did not consider the impact between the particle residual fragments and target surface. However, some investigators have suggested that secondary impacts of the fragments may accelerate the erosion. Such an effect could be studied using longer simulation of models and comparisons of numerical results with the craters caused by realistic secondary impact of particles.

(iv) Separation of the different energy components during impact. It is important to isolate the energy components during impact to determine the proportion of impact energy consumed for particle fracture, and dissipated in friction and target deformation. 
Such a partitioning might find application in optimizing the efficiency of material removal, and would help to determine the influence of particle fracture on erosion rate. The portioning could be done through numerical modeling. For example, a comparison between the ratios of rebound to initial kinetic energy in models that utilize friable particles and a rigid target and those that utilize rigid particles and a target while using zero friction coefficient would show the magnitude of kinetic energy consumed for particle fracture.

(v) Extension of models for different target and particle materials. The present study was focused on solid particle erosion of Al 6061-T6 as a typical ductile target using angular abrasives. Though, many of the realistic AJM applications use other types of ductile and brittle targets and also various abrasive types. This investigation needs to be extended for a wider range of ductile and brittle targets and particles to determine the influence of target and abrasive characteristics on different aspects of particle fracture and rebound kinematics.

(vi) Combination of fracture, embedment and erosion models. With respect to the different phenomena occurring during solid particle erosion, development of an inclusive model capable of simulating the realistic aspects such as particle embedment, fracture, and secondary impact will be a powerful tool to predict the erosion rate, roughness and geometry of the eroded surface in real solid particle erosion applications such as AJM.

(vii) Measurement of friction coefficient between the abrasive powder and target. The magnitudes of the static and dynamic friction coefficients between the abrasive particles and targets greatly affect the penetration depth and propensity for particle embedment. To the author's best knowledge, a methodology for direct measurement of this friction coefficient does not yet exist. One possible approach to this measurement might involve gluing abrasive grains to a pad that is loaded perpendicular to the surface and moved across a target surface at high velocity. Alternatively, a penshaped tool with a sample particle stuck on it could be made to rub the surface. In both cases, the tangential and normal forces would need to be measured. 


\section{Appendix A Particle Size and Shape Distributions}

As shown in Table A.1, the particle areas were found to follow a normal distribution:

$f(x \mid \mu, \sigma)=\frac{1}{\sigma \sqrt{2 \pi}} e^{\frac{-(x-\mu)^{2}}{2 \sigma^{2}}}$

The circular diameters and roundness were found to best fit a Weibull distribution of the following form:

$f(x \mid a, b)=\frac{b}{a}\left(\frac{x}{a}\right)^{b-1} e^{-(x / a)^{b}}$

Finally, the thicknesses followed a log-normal distribution:

$f(x \mid \mu, \sigma)=\frac{1}{x \sigma \sqrt{2 \pi}} e^{\frac{-(\operatorname{Ln}(x)-\mu)^{2}}{2 \sigma^{2}}} \quad ; x>0$

Table A.1 Distribution of various characterizing parameters for the utilized SiC particles.

\begin{tabular}{|c|c|c|c|c|c|}
\hline & \multicolumn{4}{|c|}{ Grit Size } \\
\hline & & 60 & 90 & 120 & 180 \\
\hline \multirow{3}{*}{ 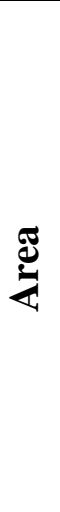 } & Mean $\left(\mu m^{2}\right)$ & 107357 & 38909 & 18507 & 6791 \\
\hline & $\begin{array}{c}\text { Standard } \\
\text { Deviation } \\
\left(\mu m^{2}\right)\end{array}$ & 32629 & 12085 & 5183 & 4008 \\
\hline & $\begin{array}{c}\text { Distribution } \\
\text { and Parameters }\end{array}$ & $\begin{array}{c}\text { Normal } \\
\mu=1.07 \times 10^{5} \\
\sigma=2.82 \times 10^{4}\end{array}$ & $\begin{array}{c}\text { Normal } \\
\mu=3.89 \times 10^{4} \\
\sigma=1.21 \times 10^{4}\end{array}$ & $\begin{array}{c}\text { Normal } \\
\mu=1.77 \times 10^{4} \\
\sigma=6.35 \times 10^{3}\end{array}$ & $\begin{array}{c}\text { Normal } \\
\mu=6.80 \times 10^{3} \\
\sigma=4.04 \times 10^{3}\end{array}$ \\
\hline \multirow{3}{*}{ 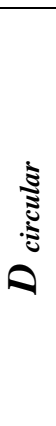 } & Mean $(\mu m)$ & 362.9 & 219.2 & 151.9 & 88.8 \\
\hline & $\begin{array}{c}\text { Standard } \\
\text { Deviation }(\mu m)\end{array}$ & 70.6 & 38.7 & 21.8 & 27.7 \\
\hline & Distribution & $\begin{array}{c}\text { Weibull } \\
a=386.34 \\
b=7.03\end{array}$ & $\begin{array}{c}\text { Weibull } \\
a=233.87 \\
b=6.89\end{array}$ & $\begin{array}{c}\text { Weibull } \\
a=161.26 \\
b=7.58\end{array}$ & $\begin{array}{c}\text { Weibull } \\
a=98.66 \\
b=3.50\end{array}$ \\
\hline$\approx$ & Mean & 0.60 & 0.59 & 0.58 & 0.56 \\
\hline
\end{tabular}




\begin{tabular}{|c|c|c|c|c|c|}
\hline & $\begin{array}{l}\text { Standard } \\
\text { Deviation }\end{array}$ & 0.11 & 0.11 & 0.12 & 0.12 \\
\hline & $\begin{array}{l}\text { Distribution } \\
\text { (Roundness) }\end{array}$ & $\begin{array}{l}\text { Weibull } \\
a=0.64 \\
b=6.51\end{array}$ & $\begin{array}{l}\text { Weibull } \\
a=0.63 \\
b=5.94\end{array}$ & $\begin{array}{l}\text { Weibull } \\
a=0.62 \\
b=5.94\end{array}$ & $\begin{array}{l}\text { Weibull } \\
a=0.61 \\
b=5.07\end{array}$ \\
\hline \multirow{3}{*}{ 号 } & $\operatorname{Mean}(\mu m)$ & 94.9 & 64.5 & 39.44 & 27.86 \\
\hline & $\begin{array}{c}\text { Standard } \\
\text { Deviation }(\mu m)\end{array}$ & 31.6 & 23.2 & 12.2 & 10.48 \\
\hline & $\begin{array}{l}\text { Distribution } \\
\text { (Thickness) }\end{array}$ & $\begin{array}{c}\text { Log normal } \\
\begin{array}{c}\mu=4.48 \\
\sigma=0.41\end{array}\end{array}$ & $\begin{array}{l}\text { Log normal } \\
\begin{array}{c}\mu=4.09 \\
\sigma=0.40\end{array}\end{array}$ & $\begin{array}{c}\text { Log normal } \\
\begin{array}{c}\mu=3.61 \\
\sigma=0.33\end{array}\end{array}$ & $\begin{array}{c}\text { Log normal } \\
\begin{array}{c}\mu=3.28 \\
\sigma=0.36\end{array}\end{array}$ \\
\hline
\end{tabular}




\section{Appendix B Modeling of Instantaneous Drag Force}

Rosendahl [167] suggested that the drag coefficient of non-spherical particles could be based on their alignment in a flow (Fig. B.1) as follows:

$C_{D}(\varphi)=C_{D, \varphi=0^{\circ}}+\left(C_{D, \varphi=90^{\circ}}-C_{D, \varphi=0^{\circ}}\right)(\sin \varphi)^{3}$

In the present work, the angular particles were approximated as two differently shaped ellipsoids for the purposes of the calculation of drag coefficient, based on the expressions for ellipsoids derived by Zastawny et al. [89] in their direct numerical simulation (DNS) framework for turbulent flow at Reynold's Numbers $R e<1000$. Shapes 1 and 2 (Fig. B.1) were used for particles with aspect ratios larger and smaller, respectively, than 1.75. The drag coefficient at a given instantaneous $\varphi$ was thus calculated using as [89]:

$C_{D}(\varphi)=C_{D, \varphi=0^{\circ}}+\left(C_{D, \varphi=90^{\circ}}-C_{D, \varphi=0^{\circ}}\right)(\sin \varphi)^{a 0}$

where

$C_{D, \varphi=0^{\circ}}=\frac{a_{1}}{R e^{a_{2}}}+\frac{a_{3}}{R e^{a_{4}}}$

$C_{D, \varphi=90^{\circ}}=\frac{a_{5}}{R e^{a_{6}}}+\frac{a_{7}}{\operatorname{Re}^{a_{8}}}$

are the drag coefficients at $\varphi=0$ and $90^{\circ}$ respectively, and $a_{0}, a_{1}, a_{2}, a_{3}, a_{4}, a_{5}, a_{6}, a_{7}, a_{8}$ are the fit parameters from Ref. [89], listed in Table B.1 for the two assumed shapes. 


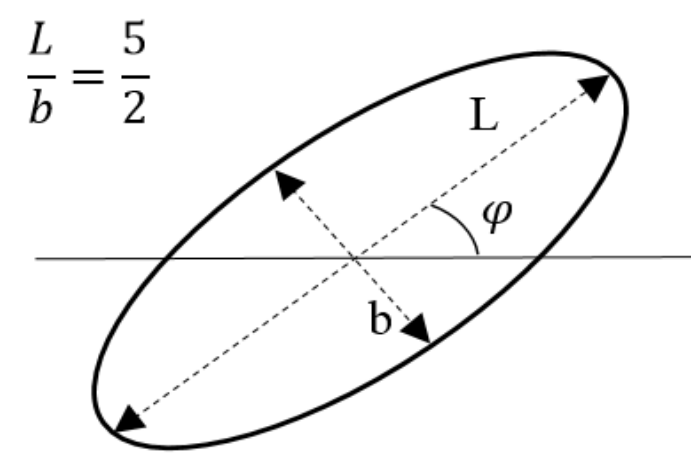

(a)

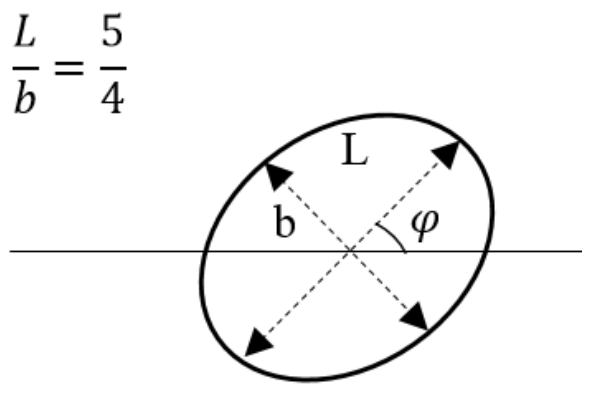

(b)

Figure B.1 Two forms of simplified non-spherical shapes utilized in this study: (a) ellipsoid 1 and (b) ellipsoid 2 [89].

Table B.1 Value of fit parameters used in eqs. B-2, B-3 and B-4 [89].

\begin{tabular}{|c|c|c|}
\hline Fit Parameter & Ellipsoid 1 & Ellipsoid 2 \\
\hline$a_{0}$ & 2.0 & 1.95 \\
\hline$a_{1}$ & 5.1 & 18.12 \\
\hline$a_{2}$ & 0.48 & 1.023 \\
\hline$a_{3}$ & 15.52 & 4.26 \\
\hline$a_{4}$ & 1.05 & 0.384 \\
\hline$a_{5}$ & 24.68 & 21.52 \\
\hline$a_{6}$ & 0.98 & 0.99 \\
\hline$a_{7}$ & 3.19 & 2.86 \\
\hline$a_{8}$ & 0.21 & 0.26 \\
\hline
\end{tabular}

Equations (B-3) and (B-4) were developed for $R e<1000$, while $R e$ in the present research could be as high as 15000 . Unfortunately, there is no data available in the literature for this range. Therefore, the $C_{D}$ in eq. (B-2) was scaled for a best fit of linear velocity with measured results. It was found that a constant factor of 3.75 resulted in predicted linear velocities that agreed fairly well with measured ones for all standoffs, pressures and particle sizes. Comparison between the calibrated drag coefficient used in the present study and the term utilized by Dehnadfar et al. [95] 
for non-rotating angular particles showed that variations of $C_{D}$ at different standoffs followed the same pattern, and that overall, they were typically within $30 \%$ of each other (Table B.2). This lends confidence to the $C_{D}$ utilized in the present study.

Table B.2 Variations of Drag coefficient versus traveled distance for 3 sample particles.

\begin{tabular}{|c|c|c|c|c|c|c|c|c|c|c|}
\hline \multirow{2}{*}{$\begin{array}{c}\text { Particle } \\
\text { Number }\end{array}$} & \multirow{2}{*}{$C_{D}$} & \multicolumn{6}{|c|}{ Distance from nozzle entrance } & \multicolumn{5}{|c|}{ Distance from nozzle exit $(\mathrm{mm})$} \\
\cline { 2 - 11 } & & 0 & 10 & 20 & 30 & 0 & 10 & 20 & 30 & 40 \\
\hline \multirow{2}{*}{1} & $*$ & 0.69 & 1.42 & 2.22 & 0.78 & 0.78 & 0.78 & 0.78 & 0.78 & 0.78 \\
\cline { 2 - 11 } & $* *$ & 0.78 & 0.75 & 0.76 & 0.78 & 0.78 & 0.78 & 0.78 & 0.78 & 0.75 \\
\hline \multirow{2}{*}{2} & $*$ & 0.81 & 1.54 & 7.90 & 1.45 & 1.45 & 1.45 & 1.45 & 1.45 & 1.45 \\
\cline { 2 - 11 } & $* *$ & 1.45 & 1.41 & 3.61 & 1.45 & 1.45 & 1.45 & 1.45 & 1.45 & 1.41 \\
\hline \multirow{2}{*}{3} & $*$ & 1.35 & 1.81 & 1.55 & 1.45 & 1.45 & 1.45 & 1.45 & 1.45 & 1.45 \\
\cline { 2 - 10 } & $* *$ & 1.44 & 1.37 & 1.41 & 1.45 & 1.45 & 1.45 & 1.45 & 1.44 & 1.37 \\
\hline
\end{tabular}

* : Drag coefficient based on calculations of present study (alignment dependent) **: Drag coefficient based on calculations of ref. [95]

An example of the fit of measured and predicted velocities utilizing this correction was shown in Fig. 2.7. 


\section{References}

[1] D.R Andrews, An analysis of solid particle erosion mechanisms, Journal of Physics D: Applied Physics 14 (1981) 1979-1991.

[2] D.R. Andrews, J.E. Field, The erosion of metals by the normal impingement of hard solid spheres, Journal of Physics D: Applied Physics 15 (1982) 571-578.

[3] D.R. Andrews, N. Horsfield, Particle collisions in the vicinity of an eroding surface, Journal of Physics D: Applied Physics 16 (1983) 525-538.

[4] H. Getu, A. Ghobeity, J.K. Spelt, M. Papini, Abrasive jet micromachining of polymethylmethacrylate, Wear 263 (2007) 1008-1015.

[5] T. H. Tsiang, survey of sand and rain erosion of composite materials, Journal of Composite Technology and Research 8(4) (1986) 154-158.

[6] G. P. Tilly, Study of behaviour of particles and materials in erosion processes, Wear 14 (1969) 63.

[7] R. P. Veerabhadra, D. H. Buckley, Angular particle impingement studies of thermoplastic materials at normal incidence, ASLE transactions 29 (3) (1986) 283-298.

[8] I. Finnie, Some reflections on the past and future of erosion, Wear 186-187 (1995) 1-10.

[9] D. Alman, J. Tylczak, J. Hawk, and M. Hebsur, Solid particle erosion behavior of an Si3N4MoSi 2 composite at room and elevated temperatures, Materials Science and Engineering A 261 (1999) 245-251.

[10] J. Zhou, S. Bahadur, Erosion-corrosion of Ti-6Al-4V in elevated temperature air environment, Wear 186-187 (1995) 332-339.

[11] R.H.M. Jafar, M. Papini, J.K. Spelt, Simulation of erosive smoothing in the abrasive jet micro-machining of glass, Journal of Materials Processing Technology 213 (12) (2013) 2254-2261.

[12] R.H.M Jafar, J.K. Spelt, M. Papini, Surface roughness and erosion rate of abrasive jet micromachined channels: Experiments and analytical model, Wear 303 (1-2) (2013) 138-145. 
[13] A.R. daCosta, R. Vilar, Erosion by solid particle impingement: experimental results with cast-iron, laser-treated surfaces, Tribology Letter 3 (1997) 379-385.

[14] L. Chen, G. Luo, K. Liu, J. Ma, B. Yao, Y. Yan, Y. Wang, Bonding of glass-based chips at low or room temperature in routine laboratory, Sensors and Actuators B: Chemical 119 (2006) 335-344.

[15] S. M. Walley, J. E. Field, The erosion and deformation of Polyethylene by solid particle impact, Philosophical transactions of the royal society of London. Series A, Mathematical and physical sciences, 321 (1558) (1987) 277-303.

[16] H. Getu, J.K. Spelt, M. Papini, Conditions leading to the embedding of angular and spherical particles during the solid particle erosion of polymers, Wear 292-293 (2012) 159-168.

[17] S. Lathabai, M. Ottmuller, I. Fernandez, Solid particle erosion behavior of thermal sprayed ceramic, metallic and polymer coatings, Wear 221 (1998) 93-108.

[18] N. Brown, M.F. Parrish, Effect of liquid nitrogen on the tensile strength of polyethylene and polytetrafluoroethylene, Journal of Polymer Science: Polymer Letters 10 (1972) 777-779.

[19] P. Beardmore, S. Rabinowitz, Low temperature crazing in amorphous polymers, Journal of Materials Science 6 (1971) 80-5.

[20] K. Mizutani, Temperature dependence of fracture toughness of poly(methyl methacrylate), Journal of Materials Science Letters 6 (1987) 915-916.

[21] H. Getu, J.K. Spelt, M. Papini, Cryogenically assisted abrasive jet micromachining of polymers, Journal of Micromechanics and Microengineering 18 (2008) 115010.

[22] W. Wu, K.C. Goretta, J.L. Routbort, Erosion of $2014 \mathrm{Al}$ reinforced with $\mathrm{SiC}$ or $\mathrm{Al}_{2} \mathrm{O}_{3}$ particles, Materials Science and Engineering: A 151 (1992) 85-95.

[23] G.L. Sheldon, J. Maji, C.T, Crowe, , Erosion of a tube by gas-particle flow, Journal of Engineering Materials and Technology, Transactions of the ASME H 99(2) (1977) 138142

[24] S. Amada, T. Hirose, T. Senda, Quantitative evaluation of residual grits under angled blasting, Surface and Coatings Technology 111 (1999) 1-9. 
[25] A.W. Momber, Y.C. Wong, E. Budidharma, R. Tjo, Surface profiling of low-carbon steel with supersonic water jets, Wear 249 (2002) 853-859.

[26] C. Toscano, G. Ahmadi, Particle removal mechanisms in cryogenic surface cleaning, The Journal of Adhesion 79 (2003) 175-201.

[27] H. Getu, J. K. Spelt and M. Papini, Reduction of particle embedding in solid particle erosion of polymers, Wear 270 (11-12) 922-928.

[28] I.M. Hutchings, Deformation of metal surfaces by the oblique impact of square plates, International Journal of Mechanical Sciences 19 (1977) 45-52.

[29] M. Papini, J. K. Spelt, Impact of rigid angular particles with fully plastic targets- Part I: Analysis, International Journal of Mechanical Sciences 42 (5) (2000) 991-1006.

[30] M. Papini, J. K. Spelt, Impact of rigid angular particles with fully plastic targets - Part II: Parametric study of erosion phenomena, International Journal of Mechanical Sciences 42 (5) (2000) 1007-1025.

[31] M. Takaffoli, M. Papini, Finite element analysis of single impacts of angular particles on ductile targets, Wear 267 (2009) 144-151.

[32] M. Takaffoli, M. Papini, Material deformation and removal due to single particle impacts on ductile material using smoothed particle hydrodynamics, Wear 274-275 (2012) 50-59.

[33] C. Marchioli, M. Picciotto, A Soldati, Influence of gravity and lift on particle velocity statistics and transfer rates in turbulent vertical channel flow, International Journal of Multiphase Flow 33 (3) (2007) 227-251.

[34] J.G.M. Kuerten, Subgrid modelling in Particle-laden channel flow, Physics of Fluids 18 (2) 2006.

[35] J.D. Kulick, J.R. Fessler, J.K. Eaton, Particle response and turbulence modification in fully developed channel flow, Journal of Fluid Mechanics 227 (1994) 109-134.

[36] G.B. Jeffery, The motion of ellipsoidal particles immersed in a viscous fluid, Proceedings of the Royal Society of London. Series A, Containing Papers of a Mathematical and Physical Character 102 (715) (1922) 161-179. 
[37] H. Brenner, The Stokes resistance of an arbitrary particle, Chemical Engineering Science 18 (1) (1963) 1-25.

[38] H. Brenner, The Stokes Resistance of an arbitrary particle -IV, arbitrary fields of flow, Chemical Engineering Science 19 (1964) 703-727.

[39] E.Y. Harper, I.D. Chang, Maximum dissipation resulting from lift in a slow viscous shear layer, Journal of Fluid Mechanics 33 (1968) 209-225.

[40] F.G. Fan, G. Ahmadi, A sublayer model for wall-deposition of ellipsoidal particles in turbulent streams, Journal of Aerosol Science 26 (1995) 813-840.

[41] H. Zhang, G. Ahmadi, F.G. Fan, J.B. McLaughlin, Ellipsoidal particles transport and deposition in turbulent channel flows, International Journal of Multiphase Flow 27 (2001) 971-1009.

[42] M. Parcheh, M.L. Brown, C.K. Aidun, On the Orientation of stiff fibers suspended in turbulent flow in a planar contraction, Journal of Fluid Mechanics 545 (2005) 245-269.

[43] O. Bernstein, M. Shapiro, Direct determination of the orientation distribution function of cylindrical particles immersed in laminar and turbulent shear flows, Journal of Aerosol Science 25 (1) (1994) 113-136.

[44] M. Mando, L. Rosendahl, On the motion of non-spherical particles at high Reynolds number, Powder Technology 202 (1-3) (2010) 1-13.

[45] P.H. Mortensen, H.I. Andersson, J.J. Gillissen, B.J. Boersma, Dynamics of prolate ellipsoidal particles in a turbulent channel flow, Physics of Fluids 20 (9) (2008) Article number 093302.

[46] J.S. Paschkewitz, Y. Dubief, C.D. Dimitropoulos, E.S.G. Shaqfeh, P. Moin, Numerical simulation of turbulent drag reduction using rigid fibers, Journal of Fluid Mechanics 518 (2004) 281-317.

[47] H. Zhang, G. Ahmadi, F-G. Fan, J.B. McLaughlin, Ellipsoidal particles transport and deposition in turbulent channel flows, International Journal of Multiphase Flow 27 (2001) 971-1009. 
[48] G. P. Tilly, W. Sage, The interaction of particle and material behavior in erosion processes, Wear 16 (1970) 447-465.

[49] A.D. Salman, C.A. Biggs, J. Fu, I. Angyal, M. Szabo, M.J. Hounslow, An experimental investigation of particle fragmentation using single particle impact studies, Powder Technology 128 (2002) 36- 46.

[50] E. Akbarzadeh, E. Elsaadawy, A.M. Sherik, J.K. Spelt, M. Papini, The solid particle erosion of 12 metals using magnetite erodent, Wear (282-283) (2012) 40-51.

[51] A. J. Sparks, I. M. Hutchings, Effects of erodent recycling in solid particle erosion testing, Wear 162-164 (1993) 139-147.

[52] A. Misra, I. Finnie, On the size effect in abrasive and erosive wear, Wear 65 (1981) 359373.

[53] J.J. Kim, S.K. Park, Solid particle erosion of SiC and SiC-TiB2 composite hot pressed, Wear 222 (1998) 114-119.

[54] A.V. Levy, The platelet mechanism of erosion of ductile metals, Wear 108 (1986) 1-21.

[55] G. Sundararajan, The depth of plastic deformation beneath eroded surfaces: the influence of impact angle and velocity, particle shape and material properties, Wear 149 (1991) 129153.

[56] L. Murugesh, R. O. Scattergood, Effect of erodent properties on the erosion of alumina, Journal of materials science 26 (1999) 5456-5466.

[57] B.R. Lawn, A.G. Evans, A model for crack initiation in elastic/plastic indentation fields, Journal of Materials Science 12 (11) (1997) 2195-2199.

[58] R. Brighenti, D. Scorza, Numerical modelling of the fracture behaviour of brittle materials reinforced with unidirectional or randomly distributed fibres, Mechanics of Materials 52 (2012) 12-27.

[59] G.M. Zhang, R.C. Batra, J. Zheng, Effect of frame size, frame type, and clamping pressure on the ballistic performance of soft body armor, Composites Part B: Engineering 39(3) (2008) 476-489. 
[60] J.W. Swegle, D.L Hicks, S.W. Attaway, Smoothed particle hydrodynamics stability analysis, Journal of Computational Physics 116 (1995) 123-134.

[61] T. Belytschko, Y. Guo, W.K. Liu, S.P. Xiao, A unified stability analysis of meshless particle methods, International Journal for Numerical Methods in Engineering 48 (2000) 13591400.

[62] T. Belytschko, S. Xiao, Stability analysis of particle methods with corrected derivatives, Computers \& Mathematics with Applications 43 (2002) 329-350.

[63] T. Rabczuk, T. Belytschko, S.P. Xiao, Stable particle methods based on Lagrangian kernels, Computer Methods in Applied Mechanics and Engineering 193 (12-14) (2004) 1035-1063.

[64] S. Hiermaier, Structures under Crash and Impact, Springer, 2008, ISBN 978-0- 387-738628

[65] W. Riedel, S. Hiermaier, K. Thoma, Transient stress and failure analysis of impact experiments with ceramics, Materials Science and Engineering B 173 (2010) 139-147.

[66] T. Belytschko, M. Tabbara, Dynamic fracture using element-free Galerkin methods, International Journal for Numerical Methods in Engineering 39 (1996) 923-938.

[67] N. Moes, J. Dolbow, T. Belytschko, A finite element method for crack growth without remeshing, International Journal for Numerical Methods in Engineering 46 (1999) 131150.

[68] T. Belytschko, Y.Y. Lu, L. Gu, Element-free Galerkin methods, International Journal of Numerical Methods in Engineering 37(2) (1994) 229-256.

[69] A. Morka, On the modelling of penetration/perforation problems, Journal of KONES Powertrain and Transport 17(1) (2010) 291-297.

[70] X. Guo, D. Fang, A. Kah Soh, H. C. Kim, J. J. Lee, Analysis of piezoelectric ceramic multilayer actuators based on an electro-mechanical coupled meshless method, Acta Mech Sinica 22(1) (2006) 34-39. 
[71] G.R. Johnson, T. J. Holmquist, An improved computational constitutive model for brittle materials, High pressure science and technology, New York, AIP (American Institute of Physics) 1994.

[72] G.R. Johnson, T. J. Holmquist, Response of boron carbide subjected to large strains, high strain rates and high pressures, Journal of Applied Physics 85 (12) (1999) 8060-8073.

[73] D.S. Duane, B. Khahn, K. Christian, Implementation and validation of the JohnsonHolmquist Ceramic Material Model in LS-Dyna. 4th European LS-DYNA Users Conference (2003).

[74] X. Quan, R.A. Clegg, M.S. Cowler, N.K. Birnbaum, C.J. Hayhurst, Numerical simulation of long rods impacting silicon carbide targets using JH-1 model, International Journal of Impact Engineering 33 (2006) 634-644.

[75] X. Guo, Y. Wei, Z. Jin, D. Guo, W. Maosen, A numerical model for optical glass cutting based on SPH method, The International Journal of Advanced Manufacturing Technology 68 (2013) 1277-1283.

[76] Y.F Wang, Z.G Yang, Finite element model of erosive wear on ductile and brittle materials, Wear 265 (2008) 871-878.

[77] C. Kaufmann, D.S. Cronin, M.J. Worswick, G. Pageau, A. Beth, Influence of material properties on the ballistic performance of ceramics for personal body armour, Shock and Vibration Journal SAV 10 (1) (2003) 51-59.

[78] M. Lee, Y.H. Yoo, Analysis of ceramic/metal armour systems, International Journal of Impact Engineering 25(9) (2001) 819-829.

[79] P. Lundberg, Interface defeat and penetration: two modes of interaction between metallic projectiles and ceramic targets, PhD dissertation, Faculty of Science and Technology, Uppsala University, Sweden (2004).

[80] N.M. Barkoula, J. Karger-kocsis, Review processes and influencing parameters of the solid particle erosion of polymers and their composites, Journal of Materials Science 37 (2002) 3807-3820. 
[81] Y.I. Oka, M. Matsumura, T. Kawabata, Relationship between surface hardness and erosion damage caused by solid particle impact, Wear 162-164 (1993) 688-695.

[82] F. Negri, E. Bedel, P. Schmitz, Experimental study of alumina particle removal from a plane surface, Journal of Adhesion 78 (2002) 79-95.

[83] H. Wadell, The coefficient of resistance as a function of Reynolds number for solids of various shapes, Journal of the Franklin institute 217(1934) 459-490.

[84] A. Hözler, M. Sommerfeld, New simple correlation formula for the drag coefficient of nonspherical particles, Powder Technology 184 (2008) 361-365.

[85] E. Loth, List of drag of non-spherical solid particles of regular and irregular shape, Powder Technology 182 (2008) 342-353.

[86] M. Mando, M.F. Lightstone, L. Rosendahl, C. Yin, H. Sørensen, Turbulence modulation in dilute particle-laden flow, International Journal of Heat and Fluid Flow 30 (2009) 331338.

[87] E.B. Christiansen, D.H. Barker, Effect of shape and density on free settling of particles at high Reynolds number, AICHE Journal 11 (1) (1965) 145.

[88] R. Clift, R. Grace, M.E. Weber, Bubbles, Drops, and Particles, Academic Press, New York, 1978.

[89] M. Zastawny, G. Mallouppas, F. Zhao, B. van Wachem, Derivation of drag and lift force and torque coefficients for non-spherical particles in flows, International Journal of Multiphase Flow 39 (2012) 227-239.

[90] A. Okagawa, S.G. Mason, The kinetics of flowing dispersions. VII. Oscillatory behavior of rods and discs in shear flow, Journal of Colloid and Interface Science 45(2) (1973) 330358.

[91] A.J. Salem, G.G. Fuller, Small angle light scattering as a probe of flow-induced particle orientation, Journal of Colloid and Interface Science 108 (1) (1985) 149-157.

[92] A. Ruff, L.K. lves, Measurement of solid particle velocity in erosive wear, Wear 35 (1975) 195-199. 
[93] D. R. Andrews, A versatile laboratory correlator with application to determine particle velocity in an erosion test, Journal of Physics E: Scientific Instruments 16 (1983) 706-709.

[94] A. Ghobeity, T. Krajac, T. Burzynski, M. Papini, J.K. Spelt, Surface evolution models in abrasive jet micromachining, Wear 264 (2008) 185-198.

[95] D. Dehnadfar, J. Friedman, M. Papini, Laser shadowgraphy measurements of abrasive particle spatial, size and velocity distributions through micro-masks used in abrasive jet micro-machining, Journal of Materials Processing Technology 212 (1) (2012) 137-149.

[96] D. Freedman, P. Diaconis, On the histogram as a density estimator: L2 theory, Probability Theory and Related Fields 57 (4) (1981) 453-476.

[97] H.Z. Li, J. Wang, J.M. Fan, Analysis and modeling of particle velocities in micro-abrasive air jet, International Journal of Machine Tools \& Manufacture 49 (2009) 850-858.

[98] J. Day, X. Huang, N.L. Richards, Examination of a grit-blasting process for thermal spraying using statistical methods, Journal of Thermal Spray Technology 14 (4) (2005) 471-479.

[99] V. Hadavi, B. Michaelsen, M. Papini, Measurements and modeling of instantaneous particle orientation within abrasive air jets and implications for particle embedding, Wear 336-337 (2015) 9-20.

[100] J.B. Zu, G.T. Burstein, I.M. Hutchings, A comparative study of slurry erosion and free-fall particle erosion of aluminum, Wear 149 (1991) 73-84.

[101]M. Junkar, B. Jurisevic, M. Fajdiga, and M. Grah, Finite element analysis of single-particle impact in abrasive water jet machining, International Journal of Impact Engineering 32 (2006) 1095-1112.

[102]K. Shimizu, T. Noguchi, H. Seitoh, M. Okada, Y. Matsubara, FEM analysis of erosive wear, Wear 250 (2001) 779-784.

[103]K. Shimizu, T. Noguchi, H. Seitoh, E. Muranaka, FEM analysis of the dependency on impact angle during erosive wear, Wear 233-235 (1999) 157-159.

[104]M. ElTobgy, E. Ng, M. Elbestawi, Finite element modeling of erosive wear, International Journal of Machine Tools and Manufacture 45 (2005) 1337-1346. 
[105] I. Finnie, Erosion of surfaces by solid particles, Wear 3 (1960) 87-103.

[106] J.G.A. Bitter, A study of erosion phenomena, Part I, Wear 6 (1963) 5-21.

[107] M. Hashish, Modified model for erosion, in Proceeding of the Seventh International Conference on Erosion by Liquid and Solid Impact, J.E. Field and J.P. Dear Eds., Cavendish Laboratory, Cambridge, England, (1987) 461-480.

[108]Y. Wang, Z. Yang, Finite element model of erosive wear on ductile and brittle materials, Wear 265 (2008) 871-878.

[109]S. Yerramareddy, S. Bahadur, Effect of operational variables, microstructure and mechanical properties on the erosion of Ti-6Al-4V, Wear 142 (1991) 253-263.

[110] G. R. Liu, M. B. Liu, Smoothed Particle Hydrodynamics: A Meshfree Particle Method. World Scientific, Singapore, 2003.

[111]M. Takaffoli, M. Papini, Numerical simulation of solid particle impacts on Al6061-T6 part I: Three-dimensional representation of angular particles, Wear 292-293 (2012) 100-110.

[112]M. Takaffoli, M. Papini, Numerical simulation of solid particle impacts on Al6061-T6 part II: Material removal mechanisms for impact of multiple angular particles, Wear 296 (2012) 648-655.

[113] D. J. Lacome, Smoothed particle hydrodynamics, Livermore Software Technology Corporation, Livermore, CA, 2001.

[114] B.M. Corbett, Numerical simulations of target hole diameters for hypervelocity impacts into elevated and room temperature bumpers, International Journal of Impact Engineering 33 (2006) 431-440.

[115]G.R. Johnson, W.H. Cook, A constitutive model and data for metals subjected to large strains, high strain rates and high temperatures, in: Proceedings of the 7th International Symposium on Ballistic (1983) 541-547.

[116] W. Dabboussi, J.A. Nemes, Modeling of ductile fracture using the dynamic punch test, International Journal of Mechanical Sciences 47 (2005) 1282-1299. 
[117] D.R. Lesuer, M.M. LeBlanc, G.J. Kay, Modeling large-strain high-rate deformation in metals, in Engineering Research, Development and Technology (UCRL 53868-98), 7-16, (Springfield, VA, National Technical Information Service).

[118] G.R. Cowper, P.S. Symonds, Strain hardening and strain rate effects in the impact loading of cantilever beams, Brown University Division of Applied Mathematics Report 28 (1957).

[119]G. Johnson, W. Cook, Fracture characteristics of three metals subjected to various strains, strain rates, temperatures and pressures, Engineering Fracture Mechanics 21 (1985) 31-48.

[120] J.O. Hallquist, LS-DYNA Keyword User's Manual Version 971, Livermore Software Technology Corporation, 2007.

[121] M. Takaffoli, Experimental and numerical study of single and multiple impacts of angular particles on ductile metals, Ph.D. thesis dissertation, Ryerson University, Canada, 2012

[122] Y.J. He, A.J.A. Winnubst, D.J. Schipper, P.M.V. Bakker, A.J. Burggraaf, H. Verweij, Friction and wear behaviour of ceramic-hardened steel couples under reciprocating sliding motion, Wear 184 (1995) 33-43.

[123] K. Miyoshi, D. H. Buckley, Friction and Wear Behavior of Single-Crystal Silicon Carbide in Contact With Titanium, NASA Technical Paper 1035 (1977).

[124] K. Miyoshi, D. H. Buckley, Friction and fracture of single crystal silicon carbide in contact with itself and with titanium, ASLE Trans. 22 (1979) 146-153.

[125] K. Miyoshi, D. H. Buckley, Friction and wear behavior of single-crystal silicon carbide in sliding contact with various metals, ASLE Trans. 22 (1979) 245-256.

[126] A. Campos-Amezcua, A. Gallegos-Munoz, C. Romero, Z. Mazur-Czerwiec, R. CamposAmezcua, Numerical investigation of the solid particle erosion rate in a steam turbine nozzle, Applied Thermal Engineering 27 (14-15) (2007) 2394-2403.

[127] A. Forder, M. Thew, D. Harrison, A numerical investigation of solid particle erosion experienced within oilfield control valves, Wear 216 (2) (1998) 184-193.

[128] G.I. Parslow, D.J. Stephenson, J.E. Strutt, S. Tetlow, Investigation of solid particle erosion in components of complex geometry, Wear 233-235 (1999) 737-745. 
[129] P. Tangestanian, M. Papini, J.K. Spelt, Starch media blast cleaning of artificially aged paint films, Wear 248 (1-2) (2001) 128-139.

[130] H. Getu, A. Ghobeity, J.K. Spelt, M. Papini, Abrasive jet micromachining of acrylic and polycarbonate polymers at oblique angles of attack, Wear 265 (5-6) (2008) 888-901.

[131] N. F. Roberts, I. M. Hutchings, Erosion of polycrystalline alumina by solid particle impact, The 5th international congress on tribology - Helsinki, Vol. 2, Finnish Society for Tribology, Espoo, Finland, (1989) 307-315.

[132] M. Papini, S. Dhar, Experimental verification of a model of erosion due to the impact of rigid single angular particles on fully-plastic targets, International Journal of Mechanical Sciences (48) (2006) 469-482.

[133] I. M. Hutchings, R. E. Winter, Particle erosion of ductile metals: a mechanism of material removal, Wear 27 (1974) 121-128.

[134] C. E. Smeltzer, M. E. Gulden, S. S. McElmury, W. A. Compton, Mechanisms of sand and dust erosion, U.S aviation material laboratories tech. Rept., (1970).

[135] P.J. Slikkerveer, F. H. in`t Veld, Model for patterned erosion, Wear 233 (1999) 377-86.

[136] R.E Winter, I. M. Hutchings, Solid particle erosion studies using single angular particles, Wear 19 (1974) 181-194.

[137] S. Nahvi, P. Shipway, D. McCartney, Effects of particle crushing in abrasion testing of steels with ash from biomass-fired power plants, Wear 267 (2009) 34-42.

[138] H. Nouraei, A. Wodoslawsky, M. Papini, J.K. Spelt, Characteristics of abrasive slurry jet micro-machining: A comparison with abrasive air jet micro-machining, Journal of Materials Processing Technology 213 (2013) 1711-1724.

[139] L. Murugesh, R. Scattergood, Effect of indentation load on fragmentation of erosion particle tips, Wear 141 (1990) 115-124.

[140] M.M. Rashid, The arbitrary local mesh refinement method: an alternative to remeshing for crack propagation analysis, Computer Methods in Applied Mechanics and Engineering 154 (1998) 133-150. 
[141] P.O. Bouchard, F. Bay, Y. Chastel, I. Tovena, Crack propagation modelling using an advanced remeshing technique, Computer Methods in Applied Mechanics and Engineering 189 (2000) 723-742.

[142] D. Xie, A.M. Waas, Discrete cohesive zone model for mixed-mode fracture using finite element analysis, Engineering Fracture Mechanics 73 (2006)1783-1796.

[143] Q. Qian, D. Xie, Analysis of mixed-mode dynamic crack propagation by interface element based on virtual crack closure technique, Engineering Fracture Mechanics 74 (2007) 807814.

[144] T. Belytschko, Y. Krongauz, D. Organ, M. Fleming, P. Krysl, Meshless methods: an overview and recent developments, Computer Methods in Applied Mechanics and Engineering 139 (1-4) (1996) 3-47.

[145] T. Belytschko, H. Chen, J. Xu, G. Zi, Dynamic crack propagation based on loss of hyperbolicity and a new discontinuous enrichment, International Journal of Numerical Methods in Engineering 58 (12) (2003) 1873-1905.

[146] L. B. Lucy, Numerical approach to testing of fission hypothesis, Astronomical Journal 82 (12) (1977) 1013-1024.

[147] R. A. Gingold, J. J. Monaghan, Smoothed particle hydrodynamics - Theory and application to non-spherical stars, Monthly Notices of the Royal Astronomical Society 181 (1977) 375389.

[148] L. D. Libersky, A.G. Petschek, Smoothed particle hydro - dynamics with strength of materials, Proc., Next Free-Lagrange Conf., W. P. Crowley, M. J. Fritts, H. E. Trease eds., Springer, Berlin, (1991) 248-257.

[149] P.W. Randles, L.D. Libersky, Smoothed Particle Hydrodynamics: Some recent improvements and applications, Computer Methods in Applied Mechanics and Engineering 139 (1996) 375-408.

[150] T. Belytschko, Y.Y. Lu, L. Gu, M. Tabbara, M. Element-free Galerkin methods for static and dynamic fracture, International Journal of Solids and Structures 32 (17-18) (1995) 2547-2570. 
[151] T. J. Holmquist, G.R Johnson, High strain rate properties and constitutive modeling of glass, 15th International Symposium on Ballistics (1995).

[152] T. Burzynski, M. Papini, Analytical models of the interference between incident and rebounding particles within an abrasive jet: Comparison with computer simulation, Wear 263 (2007) 1593-1601.

[153] M. Papini, D. Ciampini, T. Krajac, J.K. Spelt, Computer modelling of interference effects in erosion testing: effect of plume shape, Wear 255 (2003) 85-97.

[154] V. Hadavi, M. Papini, Numerical modeling of particle embedment during solid particle erosion of ductile materials, Wear 342-343 (2015) 310-321.

[155] J.O. Hallquist, LS-DYNA Theory Manual, Livermore Software Technology Corporation, 1998.

[156] C. Legallic, M. Cauret, J. Tranchet, P. Chartagnac, F. Gil, B. James, M. Pickup, A.L. Milton, W.A. Carson, A Consideration of Damage in the Interaction Between Tungsten Rod Penetrators and Ceramic Materials, 16th international symposium of ballistics, 24-27 Sep. 1996, San Francisco, CA, USA.

[157] A. Ayyar, G.A. Crawford, J.J. Williams, N. Chawla, Numerical simulation of the effect of particle spatial distribution and strength on tensile behavior of particle reinforced composites, Computational Materials Science 44 (2008) 496-506.

[158] K. M. Prewo, Tension and flexural strength of silicon carbide fibre-reinforced glass ceramics, Journal of Materials Science 21 (1986) 3590-3600.

[159] J.C. Arnold, I.M. Hutchings, Model for the erosive wear of rubber at oblique impact angles, Journal of Physics and Applied Physics 25 (1992) 222-229.

[160] M. M. Stack, N. Pungwiwat, Slurry erosion of metallics, polymers and ceramics: Particle size effects, Materials Science and Technology 15 (1999) 337-344.

[161] F.O. Harold, The size reduction paradox, Industrial \& Engineering Chemistry Research 55 (1963) 46-52.

[162] I. Finnie, Some observations on the erosion of ductile metals, Wear 19 (1972) 81-91. 
[163] P. H. Shipway, I. M. Hutchings, The influence of particle properties on the erosive wear of sintered boron carbide, Wear 149 (1991) 85-98.

[164] J. E. Goodwin, W. Sage, G. P. Tilly, Study of erosion by solid particles, Proceedings of the Institution of Mechanical Engineers 184 (1969) 279-292.

[165] C.T. Morrison, R.O. Scattergood, J.L. Routbort, Erosion of 304 stainless steel, Wear 111 (1986) 1-13.

[166] S. W. Date, S. Malkin, Effect of grit size on abrasion with coated abrasives, Wear 40 (1976) 223-235.

[167] L. Rosendahl, Using a multi-parameter particle shape description to predict the motion of non-spherical particle shapes in swirling flow, Applied Mathematical Modelling 24 (1) (2000) 11-25. 